Supplementary Information

\title{
Transition-Metal-Free Cascade Synthesis of 4-Quinolones: Umpolung of Michael Acceptors via Ene Reaction with Arynes
}

R. Santhosh Reddy, ${ }^{+}$Chandraiah Lagishetti, ${ }^{+}$I. N. Chaithanya Kiran, Hengyao You and Yun $\mathrm{He}^{*}$

$\underline{\text { S.No }}$ Table of Contents $\quad$ Page

1 General information $\quad$ S2

2 Literature reports for 4-quinolones and intermolecular aryne-ene reaction S3

3 Synthesis and characterization of $\mathrm{AMBH}$ adducts $\quad \mathrm{S} 4$

$4 \quad$ General procedure for the optimization of the reaction conditions $\quad$ S12

5 Synthesis and characterization of 4-quinolones via ene reaction S14

6 Synthesis and characterization of 4-quinolones via cascade reaction S18

7 Control and cross-over experiments $\quad$ S33

8 Scale-up experiment $\quad$ S38

9 Reaction with internal olefin SI-9 S39

10 References $\quad$ S41

11 Spectra $\quad S 42$ 


\section{General Information}

Unless otherwise specified, all reactions were carried out under an atmosphere of argon in flame-dried reaction vessels with Teflon screw caps. Reaction temperatures are reported as the temperature of the bath surrounding the reaction vessel. $25^{\circ} \mathrm{C}$ corresponds to the room temperature of the lab when the experiments were carried out. THF and $\mathrm{CH}_{3} \mathrm{CN}$ were freshly purified by distillation over Na-benzophenone and calcium hydride respectively. 18-Crown-6 was recrystallized from dry $\mathrm{CH}_{3} \mathrm{CN}$ and $\mathrm{KF}$ was dried by heating at $110{ }^{\circ} \mathrm{C}$ for $12 \mathrm{~h}$ and left to cool under argon. Reactions were monitored by analytical thin-layer chromatography (TLC) on Merck silica gel $60 \mathrm{~F}_{254}$ plates $(0.25 \mathrm{~mm})$, visualized by ultraviolet light. ${ }^{1} \mathrm{H}$ NMR spectra were obtained on an Agilent 400MR or 600MR DD2 spectrometer at ambient temperature.

Data were reported as follows: chemical shift on the $\delta$ scale using residual proton solvent as internal standard [ 00.00 (TMS)], multiplicity $(\mathrm{br}=$ broad, $\mathrm{s}=$ singlet, $\mathrm{d}=$ doublet, $\mathrm{t}=$ triplet, $\mathrm{q}=$ quartet, $\mathrm{m}=$ multiplet), integration, and coupling constant(s) in hertz. ${ }^{13} \mathrm{C} \mathrm{NMR}$ spectra were obtained with proton decoupling on an Agilent 400MR or 600MR DD2 (101 MHz or $151 \mathrm{MHz}$ ) spectrometer and were reported in ppm with residual solvent for internal standard [ $\delta$ 77.0 $\left(\mathrm{CDCl}_{3}\right)$ ]. High resolution mass spectra were obtained on a Bruker SolariX 7.0T spectrometer. Melting point was determined by WRS-2A Digital Melting Point Apparatus. IR spectra were recorded on a Bruker 100 FT-IR spectrometer and are reported in terms of frequency of absorption $\left(\mathrm{cm}^{-1}\right)$. The 2(trimethylsilyl)phenyl trifluoromethane sulfonate $1 \mathrm{a}$ and the other symmetric and unsymmetric aryne precursors were synthesized following literature procedure. ${ }^{1}$ 


\section{Literature reports for 4-quinolones and intermolecular aryne-ene reaction}

Various methods have been developed for the synthesis of this valuable scaffold, ${ }^{2}$ relying mostly upon traditional cyclocondensation reactions such as the Conrad-Limpach, ${ }^{3}$ Niementowski, ${ }^{4}$ or Camps cyclizations. ${ }^{5}$ However, these methods are limited by harsh reaction conditions, unsatisfactory yields, poor regioselectivities and lack generality. In this respect, several improved procedures were reported to provide 3- or 2-substituted-4-quinolones under milder conditions comprising of (i) tandem reaction from $\beta$-ketonaldehydes and amines; ${ }^{6 a}$ (ii) oxidative Mannich reaction from $\mathrm{N}$-arylmethyl-2-aminophenylketones ${ }^{6 b}$ and (iii) transition-metal catalyzed reactions (Scheme 1). ${ }^{7}$

Improved Methods:

a)
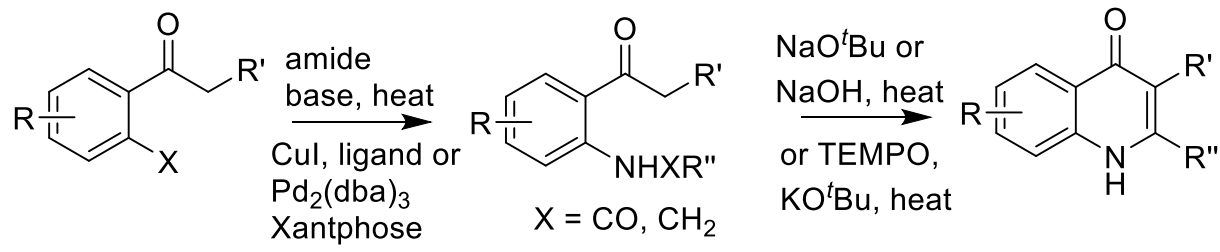

b)<smiles>[R]C#CC(=O)c1cc[R]#cc1[X]</smiles>

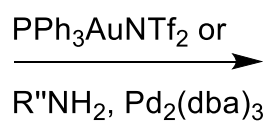
$\mathrm{X}=\mathrm{NH}_{2}$ or $\mathrm{Br}$, I<smiles>[R]C=C1C=C[R]=CC2=CC([R])=C([R])N([R7])C1=C2</smiles>

This work

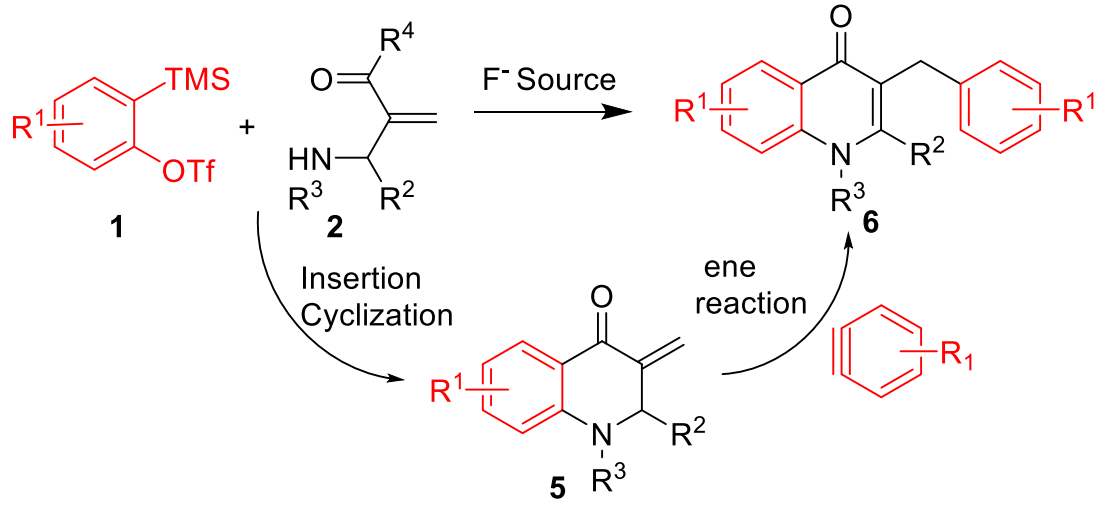

Scheme 1. Synthetic approaches toward 4-quinolones 


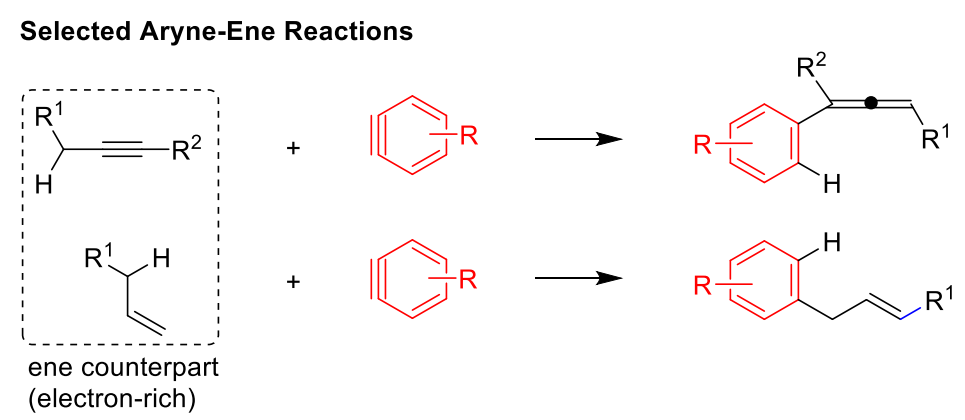

Present Method: Inverse Electron Demand Ene Reaction

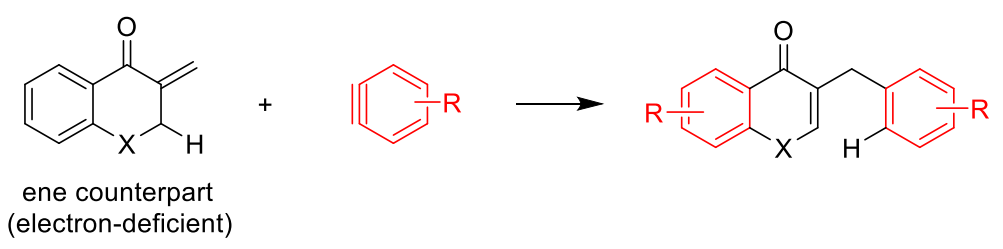

Scheme 2. Previous and Present Intermolecular Aryne-Ene Reactions

\section{Synthesis and characterization of AMBH adducts}

A general scheme that was followed for the preparation of aza-Morita-Baylis-Hillman (AMBH) adducts is provided below. Only the spectroscopic details for the unreported AMBH adducts are provided. For the reported compounds appropriate citations in which they appear are included.

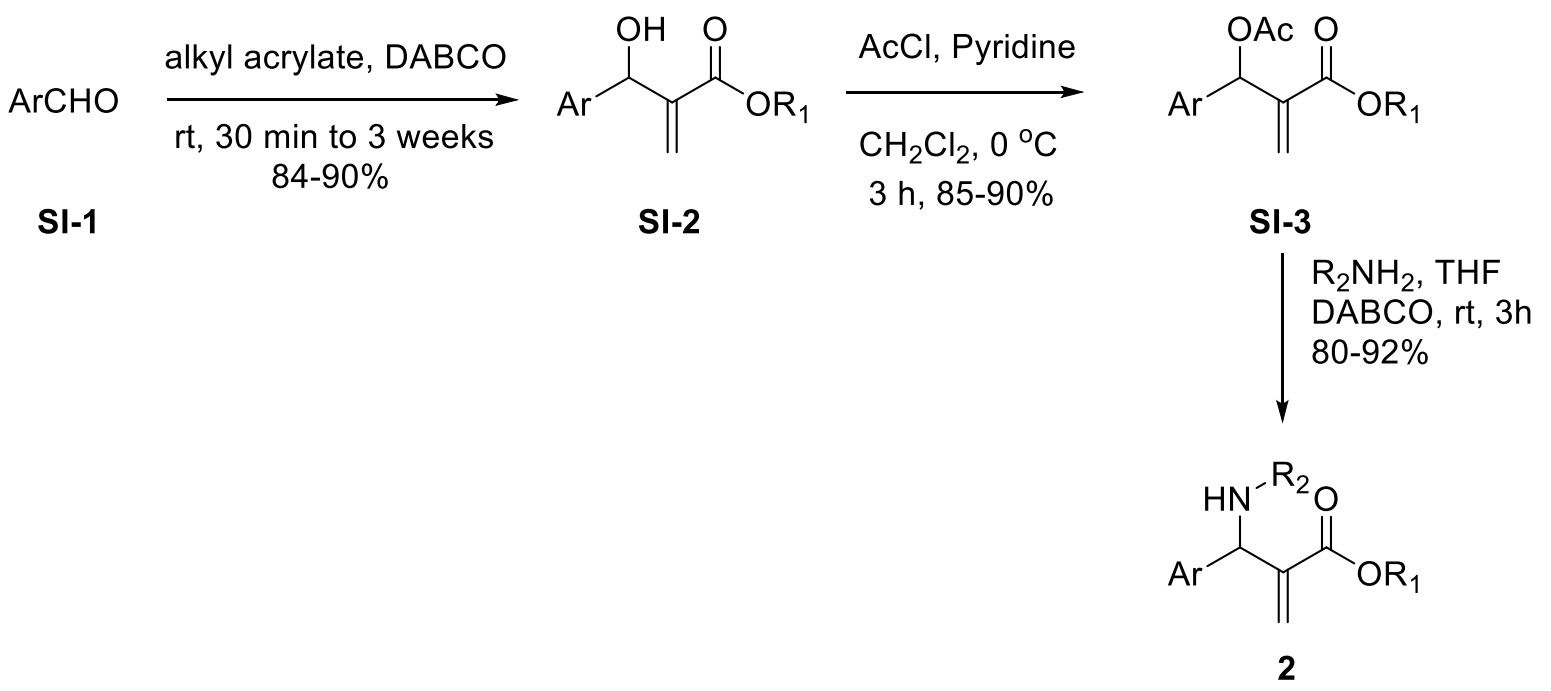

1. The AMBH adducts were prepared according to the literature procedure. ${ }^{8}$

Methyl 2-(hydroxy(phenyl)methyl)acrylate (2a) ${ }^{9}$ 
Methyl 2-((benzylamino)(phenyl)methyl)acrylate $(2 p)^{10}$

Methyl 2-((phenylamino)(p-tolyl)methyl)acrylate (2r) ${ }^{9}$

Methyl 2-((4-fluorophenyl)(phenylamino)methyl)acrylate (2s) ${ }^{10}$

Methyl 2-((phenylamino)(4-(trifluoromethyl)phenyl)methyl)acrylate (2t) ${ }^{10}$

Methyl 2-((3-chlorophenyl)(phenylamino)methyl)acrylate (2z) ${ }^{11}$

Methyl 2-((2-chlorophenyl)(phenylamino)methyl)acrylate (2ab) ${ }^{10}$

Methyl 2-((phenylamino)(thiophen-2-yl)methyl)acrylate (2ae $)^{12}$

Methyl 2-(naphthalen-1-yl(phenylamino)methyl)acrylate (2af) ${ }^{12}$

Methyl 2-((phenylamino)methyl)acrylate (2ag) $)^{13}$

Methyl 2-(((3-bromophenyl)amino)(phenyl)methyl)acrylate (2h)

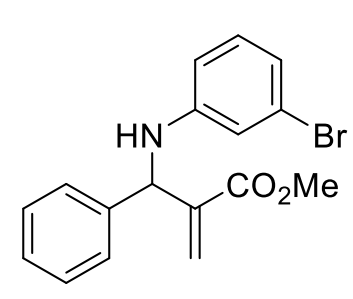

$2 h$

Pale yellow oil $\left(0.37 \mathrm{~g}, 81 \%\right.$ yield); $\mathrm{R}_{f}=0.6$ (silica, EtOAc:hexanes, 0.5:9.5); IR (film) $v_{\max } 3415,3015,1718,1630,921,774 \mathrm{~cm}^{-1} ;{ }^{1} \mathrm{H}$ NMR $\left(400 \mathrm{MHz}, \mathrm{CDCl}_{3}\right) \delta=7.43-7.29(\mathrm{~m}, 5 \mathrm{H}), 7.08-6.96(\mathrm{~m}, 1 \mathrm{H}), 6.85(\mathrm{~d}$, $J=7.9 \mathrm{~Hz}, 1 \mathrm{H}), 6.73(\mathrm{q}, J=2.2 \mathrm{~Hz}, 1 \mathrm{H}), 6.50(\mathrm{~d}, J=8.2 \mathrm{~Hz}, 1 \mathrm{H}), 6.42$ (s, 1H), $5.94(\mathrm{~s}, 1 \mathrm{H}), 5.41(\mathrm{~d}, J=5.3 \mathrm{~Hz}, 1 \mathrm{H}), 4.32(\mathrm{~d}, J=5.8 \mathrm{~Hz}, 1 \mathrm{H})$, $3.72(\mathrm{~d}, J=1.8 \mathrm{~Hz}, 3 \mathrm{H}) ;{ }^{13} \mathrm{C} \mathrm{NMR}\left(101 \mathrm{MHz}, \mathrm{CDCl}_{3}\right) \delta=166.3,147.8,139.9,139.4,130.3$, $128.7,127.8,127.3,126.2,123.0,120.5,115.9,111.8,58.6,51.8$ ppm; HRMS (m/z): $\left[\mathrm{M}+\mathrm{Na}^{+}\right.$ calcd for $\mathrm{C}_{17} \mathrm{H}_{16} \mathrm{BrNO}_{2} \mathrm{Na}^{+} 368.0256$, Found 368.0253 .

Methyl 2-(((4-bromophenyl)amino)(phenyl)methyl)acrylate (2i) 


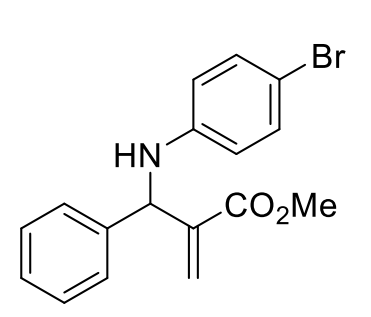

$\mathbf{2 i}$

Pale yellow oil $\left(0.38 \mathrm{~g}, 84 \%\right.$ yield); $\mathrm{R}_{f}=0.6$ (silica, EtOAc:hexanes, 0.5:9.5); IR (film) $v_{\max } 3409,3018,1714,1648,943,776 \mathrm{~cm}^{-1} ;{ }^{1} \mathrm{H}$ NMR $\left(400 \mathrm{MHz}, \mathrm{CDCl}_{3}\right) \delta=7.46-7.28(\mathrm{~m}, 5 \mathrm{H}), 7.24(\mathrm{~d}, J=8.4 \mathrm{~Hz}, 2 \mathrm{H}), 6.46$ (d, J = 8.5 Hz, 2H), $6.40(\mathrm{~s}, 1 \mathrm{H}), 5.92(\mathrm{~s}, 1 \mathrm{H}), 5.38(\mathrm{~s}, 1 \mathrm{H}), 4.24(\mathrm{~s}, 1 \mathrm{H})$, $3.72(\mathrm{~s}, 3 \mathrm{H}) ;{ }^{13} \mathrm{C}$ NMR $\left(101 \mathrm{MHz}, \mathrm{CDCl}_{3}\right) \delta=166.4,145.5,140.0,139.6$, 131.8, 128.7, 127.9, 127.3, 126.2, 114.9, 109.5, 58.8, 51.9 ppm; HRMS (m/z): $[\mathrm{M}+\mathrm{Na}]^{+}$calcd for $\mathrm{C}_{17} \mathrm{H}_{16} \mathrm{BrNO}_{2} \mathrm{Na}^{+}$368.0256, Found 368.0254.

\section{Methyl 2-(((4-ethylphenyl)amino)(phenyl)methyl)acrylate (2j)}

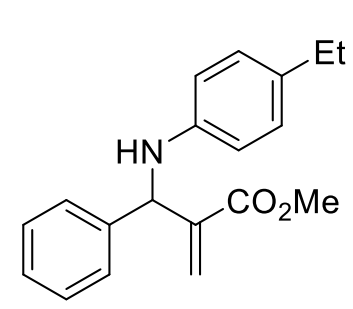

2j

Pale yellow oil $\left(0.25 \mathrm{~g}, 85 \%\right.$ yield); $\mathrm{R}_{f}=0.6$ (silica, EtOAc:hexanes, 0.5:9.5); IR (film) $v_{\max } 3414,2995,1719,1640,837,694 \mathrm{~cm}^{-1} ;{ }^{1} \mathrm{H}$ NMR $\left(400 \mathrm{MHz}, \mathrm{CDCl}_{3}\right) \delta=7.47-7.29(\mathrm{~m}, 5 \mathrm{H}), 7.11-7.01(\mathrm{~m}, 2 \mathrm{H}), 6.63-$ $6.55(\mathrm{~m}, 2 \mathrm{H}), 6.45(\mathrm{~s}, 1 \mathrm{H}), 6.04(\mathrm{~s}, 1 \mathrm{H}), 5.46(\mathrm{~s}, 1 \mathrm{H}), 4.13(\mathrm{~s}, 1 \mathrm{H}), 3.75$ (s, 3H), 2.60 (tt, J = 7.7, $4.4 \mathrm{~Hz}, 2 \mathrm{H}), 1.25(\mathrm{td}, J=7.6,2.0 \mathrm{~Hz}, 3 \mathrm{H}) ;{ }^{13} \mathrm{C}$ $\operatorname{NMR}\left(101 \mathrm{MHz}, \mathrm{CDCl}_{3}\right) \delta=166.6,144.5,140.6,140.0,133.5,128.6,128.3,127.6,127.4$, 126.0, 113.4, 59.0, 51.7, 27.8, 15.8 ppm; HRMS (m/z): $[\mathrm{M}+\mathrm{Na}]^{+}$calcd for $\mathrm{C}_{19} \mathrm{H}_{21} \mathrm{NO}_{2} \mathrm{Na}^{+}$ 318.1464, Found 318.1464.

\section{Methyl 2-(((4-ethylphenyl)amino)(phenyl)methyl)acrylate (2k)}<smiles>C=C(OC)C(Nc1ccccc1)c1ccc(Cl)cc1</smiles>

2k

Pale yellow oil $\left(0.26 \mathrm{~g}, 85 \%\right.$ yield); $\mathrm{R}_{f}=0.6$ (silica, EtOAc:hexanes, 0.5:9.5); IR (film) $v_{\max } 3430,3012,1714,1642,934,780 \mathrm{~cm}^{-1} ;{ }^{1} \mathrm{H}$ $\operatorname{NMR}\left(400 \mathrm{MHz}, \mathrm{CDCl}_{3}\right) \delta=7.37-7.30(\mathrm{~m}, 4 \mathrm{H}), 7.20(\mathrm{t}, J=7.7 \mathrm{~Hz}$, 2H), $6.78(\mathrm{t}, J=7.3 \mathrm{~Hz}, 1 \mathrm{H}), 6.61(\mathrm{~d}, J=8.0 \mathrm{~Hz}, 2 \mathrm{H}), 6.43(\mathrm{~d}, J=5.1$ Hz, 1H), 5.97 (d, J = 5.4 Hz, 1H), $5.43(\mathrm{~d}, J=5.3 \mathrm{~Hz}, 1 \mathrm{H}), 4.22$ (s, 
$1 \mathrm{H}), 3.74(\mathrm{~s}, 3 \mathrm{H}) ;{ }^{13} \mathrm{C} \mathrm{NMR}\left(101 \mathrm{MHz}, \mathrm{CDCl}_{3}\right) \delta=166.3,146.3,139.7,139.0,133.4,129.1$, 128.8, 128.7, 126.5, 118.0, 113.4, 58.2, $51.9 \mathrm{ppm}$; HRMS $(\mathrm{m} / \mathrm{z}):[\mathrm{M}+\mathrm{Na}]^{+}$calcd for $\mathrm{C}_{17} \mathrm{H}_{16} \mathrm{CINO}_{2} \mathrm{Na}^{+}$324.0761, Found 324.0762.

\section{Methyl 2-(((4-ethylphenyl)amino)(phenyl)methyl)acrylate (2l)}

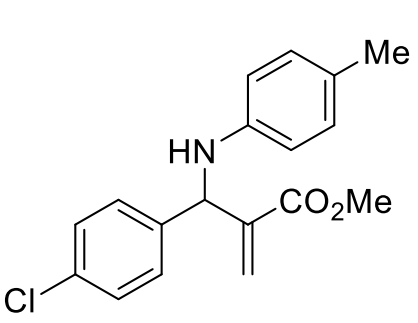

2l

Yellow oil (0.28 g, 90\% yield); $\mathrm{R}_{f}=0.6$ (silica, EtOAc:hexanes, 0.5:9.5); IR (film) $v_{\max } 3412,2998,1716,1638,890,675 \mathrm{~cm}^{-1} ;{ }^{1} \mathrm{H}$ $\operatorname{NMR}\left(400 \mathrm{MHz}, \mathrm{CDCl}_{3}\right) \delta=7.35(\mathrm{~d}, J=1.6 \mathrm{~Hz}, 4 \mathrm{H}), 7.01(\mathrm{~d}, J=$ $7.9 \mathrm{~Hz}, 2 \mathrm{H}), 6.61-6.49(\mathrm{~m}, 2 \mathrm{H}), 6.43(\mathrm{~s}, 1 \mathrm{H}), 5.98(\mathrm{~d}, J=1.7 \mathrm{~Hz}$, $1 \mathrm{H}), 5.40(\mathrm{~s}, 1 \mathrm{H}), 4.10(\mathrm{~s}, 1 \mathrm{H}), 3.74(\mathrm{~d}, J=1.4 \mathrm{~Hz}, 3 \mathrm{H}), 2.27$ (s, $3 \mathrm{H}) ;{ }^{13} \mathrm{C}$ NMR $\left(101 \mathrm{MHz}, \mathrm{CDCl}_{3}\right) \delta=166.3,144.1,139.8,139.2,133.3,129.6,128.7,127.2$, 126.5, 113.5, 58.5, 51.8, 20.3 ppm; HRMS (m/z): $[\mathrm{M}+\mathrm{Na}]^{+}$calcd for $\mathrm{C}_{18} \mathrm{H}_{18} \mathrm{CINO}_{2} \mathrm{Na}^{+} 338.0918$, Found 338.0917.

\section{Methyl 2-((4-chlorophenyl)((4-fluorophenyl)amino)methyl)acrylate (2m)}

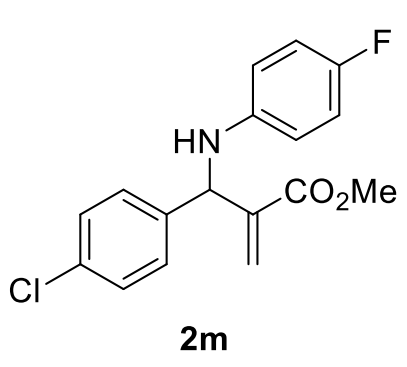

142.7, 139.7, 138.9, 133.5, 128.8, 128.7, 126.6, 115.6, 115.4, 114.3, 114.3, 58.8, 51.9 ppm; HRMS (m/z): $[\mathrm{M}+\mathrm{Na}]^{+}$calcd for $\mathrm{C}_{17} \mathrm{H}_{15} \mathrm{CIFNO}_{2} \mathrm{Na}^{+} 342.0667$, Found 342.0667 .

\section{Methyl 2-((4-chlorophenyl)((4-chlorophenyl)amino)methyl)acrylate (2n)}<smiles>C=C(COC)C(Nc1ccc(Cl)cc1)c1ccc(Cl)cc1</smiles>

Yellow oil (0.27 g, 82\% yield); $R_{f}=0.6$ (silica, EtOAc:hexanes, 0.5:9.5); IR (film) $v_{\max } 3424,3046,1719,1658,931,760 \mathrm{~cm}^{-1} ;{ }^{1} \mathrm{H}$ 
$\operatorname{NMR}\left(400 \mathrm{MHz}, \mathrm{CDCl}_{3}\right) \delta=7.31(\mathrm{~s}, 4 \mathrm{H}), 7.10(\mathrm{~d}, J=8.3 \mathrm{~Hz}, 2 \mathrm{H}), 6.50(\mathrm{~d}, J=8.3 \mathrm{~Hz}, 2 \mathrm{H}), 6.40$ (s, 1H), $5.90(\mathrm{~s}, 1 \mathrm{H}), 5.35(\mathrm{~d}, J=5.3 \mathrm{~Hz}, 1 \mathrm{H}), 4.24(\mathrm{~d}, J=5.4 \mathrm{~Hz}, 1 \mathrm{H}), 3.72(\mathrm{~s}, 3 \mathrm{H}) ;{ }^{13} \mathrm{C}$ NMR $\left(101 \mathrm{MHz}, \mathrm{CDCl}_{3}\right) \delta=166.2,144.9,139.4,138.6,133.6,128.9,128.8,128.7,126.7,122.6$, 114.5, 58.4, 52.0 ppm; HRMS (m/z): $[\mathrm{M}+\mathrm{Na}]^{+}$calcd for $\mathrm{C}_{17} \mathrm{H}_{15} \mathrm{Cl}_{2} \mathrm{NO}_{2} \mathrm{Na}^{+}$358.0372, Found 358.0372.

\section{Methyl 2-((4-chlorophenyl)((4-methoxyphenyl)amino)methyl)acrylate (20)}

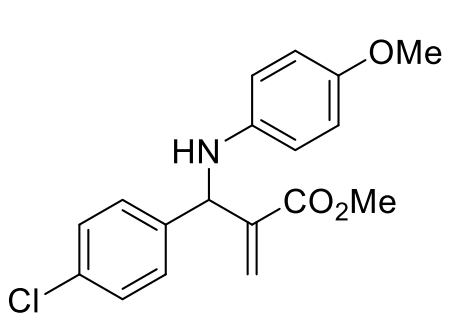

20

Pale yellow oil $\left(0.28 \mathrm{~g}, \quad 85 \%\right.$ yield); $R_{f}=0.6 \quad$ (silica, EtOAc:hexanes, 0.5:9.5); IR (film) $v_{\max } 3432,3037,1716,1679$, 893, $746 \mathrm{~cm}^{-1} ;{ }^{1} \mathrm{H}$ NMR $\left(400 \mathrm{MHz}, \mathrm{CDCl}_{3}\right) \delta=7.37-7.28(\mathrm{~m}$, 4H), $6.78(\mathrm{~d}, J=8.6 \mathrm{~Hz}, 2 \mathrm{H}), 6.56(\mathrm{~d}, J=8.6 \mathrm{~Hz}, 2 \mathrm{H}), 6.41(\mathrm{~s}$, 1H), $5.95(\mathrm{~s}, 1 \mathrm{H}), 5.35(\mathrm{~s}, 1 \mathrm{H}), 4.02(\mathrm{~s}, 1 \mathrm{H}), 3.74(\mathrm{~s}, 3 \mathrm{H}), 3.72(\mathrm{~s}$,

$3 \mathrm{H}) ;{ }^{13} \mathrm{C}$ NMR $\left(101 \mathrm{MHz}, \mathrm{CDCl}_{3}\right) \delta=166.3,152.3,140.5,139.9,139.2,133.2,128.7,128.6$, 126.4, 114.7, 114.6, 59.0, 55.5, 51.8 ppm; HRMS (m/z): $[\mathrm{M}+\mathrm{Na}]^{+}$calcd for $\mathrm{C}_{18} \mathrm{H}_{18} \mathrm{CINO}_{3} \mathrm{Na}^{+}$ 354.0867, Found 354.0865.

\section{Methyl 2-((benzylamino)(4-chlorophenyl)methyl)acrylate (2q)}<smiles>C=C(OC)C(NCc1ccccc1)c1ccc(Cl)cc1</smiles>

$2 q$

Yellow oil $\left(0.27 \mathrm{~g}, 86 \%\right.$ yield); $\mathrm{R}_{f}=0.6$ (silica, EtOAc:hexanes, 0.5:9.5); IR (film) $v_{\max } 3441,3021,1718,1692,921,766 \mathrm{~cm}^{-1} ;{ }^{1} \mathrm{H}$ $\operatorname{NMR}\left(400 \mathrm{MHz}, \mathrm{CDCl}_{3}\right) \delta=7.36-7.25(\mathrm{~m}, 9 \mathrm{H}), 6.38(\mathrm{~d}, J=1.4 \mathrm{~Hz}$,

$1 \mathrm{H}), 6.00(\mathrm{t}, J=1.3 \mathrm{~Hz}, 1 \mathrm{H}), 4.68(\mathrm{~s}, 1 \mathrm{H}), 3.75-3.66(\mathrm{~m}, 5 \mathrm{H}) ;{ }^{13} \mathrm{C}$ $\operatorname{NMR}\left(101 \mathrm{MHz}, \mathrm{CDCl}_{3}\right) \delta=166.5,141.5,140.0,139.9,132.9,129.0,128.4,128.3,128.0$ 127.0, 125.7, 61.4, 51.8, 51.6 ppm; HRMS (m/z): $[\mathrm{M}+\mathrm{Na}]^{+}$calcd for $\mathrm{C}_{18} \mathrm{H}_{18} \mathrm{CINO}_{2} \mathrm{Na}^{+} 338.0918$, Found 338.0916.

\section{Methyl 2-((phenylamino)(4-(trifluoromethoxy)phenyl)methyl)acrylate (2u)}




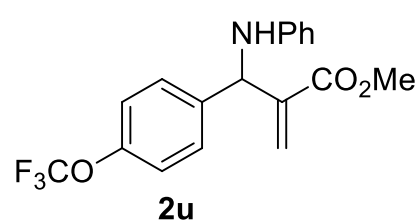

Yellow oil $\left(0.291 \mathrm{~g}, 83 \%\right.$ yield); $\mathrm{R}_{f}=0.6$ (silica, EtOAc:hexanes, 0.5:9.5); IR (film) $v_{\max } 3456,3014,1714,1631,1495,1089,1039 \mathrm{~cm}^{-}$ '; ${ }^{1} \mathrm{H} \mathrm{NMR}\left(400 \mathrm{MHz}, \mathrm{CDCl}_{3}\right) \delta=7.40(\mathrm{~d}, J=8.5 \mathrm{~Hz}, 2 \mathrm{H}), 7.16(\mathrm{~m}$, 4H), $6.73(\mathrm{t}, J=7.3 \mathrm{~Hz}, 1 \mathrm{H}), 6.56(\mathrm{~d}, J=7.9 \mathrm{~Hz}, 2 \mathrm{H}), 6.40(\mathrm{~s}, 1 \mathrm{H}), 5.95(\mathrm{~s}, 1 \mathrm{H}), 5.42(\mathrm{~s}, 1 \mathrm{H})$, 4.18 (brs, $1 \mathrm{H}), 3.69(\mathrm{~s}, 3 \mathrm{H}) ;{ }^{13} \mathrm{C} \mathrm{NMR}\left(101 \mathrm{MHz}, \mathrm{CDCl}_{3}\right) \delta=166.4,148.6,146.3,139.7,139.3$, 129.2, 128.9, 126.7, 121.1, 118.1, 113.4, 58.2, 51.9 ppm; HRMS (m/z): $[\mathrm{M}+\mathrm{Na}]^{+}$calcd for $\mathrm{C}_{18} \mathrm{H}_{16} \mathrm{~F}_{3} \mathrm{NO}_{3} \mathrm{Na}^{+}$374.0974, Found 374.0971.

\section{Methyl 2-((4-nitrophenyl)(phenylamino)methyl)acrylate (2v)}

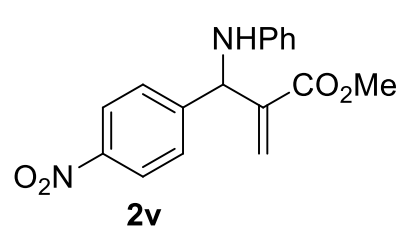

Brown oil $\left(0.262 \mathrm{~g}, 84 \%\right.$ yield); $\mathrm{R}_{f}=0.6$ (silica, EtOAc:hexanes, 0.5:9.5); IR (film) $v_{\max } 3428,3030,1717,1647,1550,1345,776 \mathrm{~cm}^{-1}$; ${ }^{1} \mathrm{H}$ NMR $\left(400 \mathrm{MHz}, \mathrm{CDCl}_{3}\right) \delta=8.17(\mathrm{~d}, J=8.7 \mathrm{~Hz}, 2 \mathrm{H}), 7.57(\mathrm{~d}, J=$ $8.5 \mathrm{~Hz}, 2 \mathrm{H}), 7.16(\mathrm{t}, J=7.6 \mathrm{~Hz}, 2 \mathrm{H}), 6.75(\mathrm{t}, J=7.3 \mathrm{~Hz}, 1 \mathrm{H}), 6.59(\mathrm{~d}, J=8.1 \mathrm{~Hz}, 2 \mathrm{H}), 6.45(\mathrm{~s}$, $1 \mathrm{H}), 5.97(\mathrm{~s}, 1 \mathrm{H}), 5.51(\mathrm{~s}, 1 \mathrm{H}), 4.33(\mathrm{brs}, 1 \mathrm{H}), 3.71(\mathrm{~s}, 3 \mathrm{H}) ;{ }^{13} \mathrm{C} \mathrm{NMR}\left(101 \mathrm{MHz}, \mathrm{CDCl}_{3}\right) \delta=$ 166.0, 148.0, 147.3, 146.0, 139.2, 129.2, 128.1, 127.8, 123.8, 118.5, 113.6, 58.5, 52.1 ppm; HRMS (m/z): $[\mathrm{M}+\mathrm{Na}]^{+}$calcd for $\mathrm{C}_{17} \mathrm{H}_{16} \mathrm{~N}_{2} \mathrm{O}_{4} \mathrm{Na}^{+}$335.1002, Found 335.1001.

\section{Methyl 2-((4-cyanophenyl)(phenylamino)methyl)acrylate (2w)}

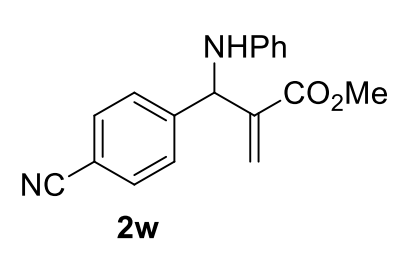

Yellow oil $\left(0.254 \mathrm{~g}, 87 \%\right.$ yield); $\mathrm{R}_{f}=0.6$ (silica, EtOAc:hexanes, 0.5:9.5); IR (film) $v_{\max } 3397,3030,2245,1719,1597,1149,776 \mathrm{~cm}^{-1}$; ${ }^{1} \mathrm{H} \mathrm{NMR}\left(400 \mathrm{MHz}, \mathrm{CDCl}_{3}\right) \delta=7.61(\mathrm{~d}, J=7.9 \mathrm{~Hz}, 2 \mathrm{H}), 7.51(\mathrm{~d}, J=$ $7.9 \mathrm{~Hz}, 2 \mathrm{H}), 7.16(\mathrm{t}, J=7.6 \mathrm{~Hz}, 2 \mathrm{H}), 6.75(\mathrm{t}, J=7.2 \mathrm{~Hz}, 1 \mathrm{H}), 6.57(\mathrm{~d}, J=7.8 \mathrm{~Hz}, 2 \mathrm{H}), 6.43(\mathrm{~s}$, $1 \mathrm{H}), 5.94(\mathrm{~s}, 1 \mathrm{H}), 5.46(\mathrm{~s}, 1 \mathrm{H}), 4.29(\mathrm{brs}, 1 \mathrm{H}), 3.71(\mathrm{~s}, 3 \mathrm{H}) ;{ }^{13} \mathrm{C} \mathrm{NMR}\left(101 \mathrm{MHz}, \mathrm{CDCl}_{3}\right) \delta=$ 166.0, 146.0, 139.3, 132.5, 129.2, 128.0, 127.6, 118.5, 113.5, 111.4, 58.7, 52.1 ppm; HRMS (m/z): $[\mathrm{M}+\mathrm{Na}]^{+}$calcd for $\mathrm{C}_{18} \mathrm{H}_{16} \mathrm{~N}_{2} \mathrm{O}_{2} \mathrm{Na}^{+} 315.1104$, Found 315.1103. 


\section{Methyl 2-((3,5-difluorophenyl)(phenylamino)methyl)acrylate (2x)}

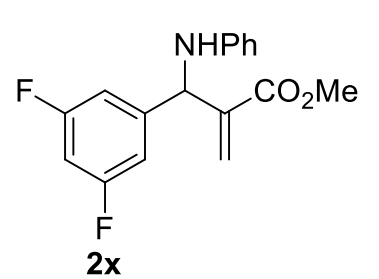

Yellow oil $\left(0.260 \mathrm{~g}, 86 \%\right.$ yield); $\mathrm{R}_{f}=0.6$ (silica, EtOAc:hexanes, 0.5:9.5);

IR (film) $v_{\max } 3406,3052,1716,1597,1436,1092,1028 \mathrm{~cm}^{-1} ;{ }^{1} \mathrm{H}$ NMR $\left(400 \mathrm{MHz}, \mathrm{CDCl}_{3}\right) \delta=7.21(\mathrm{t}, J=7.8 \mathrm{~Hz}, 2 \mathrm{H}), 6.98(\mathrm{~d}, J=6.4 \mathrm{~Hz}, 2 \mathrm{H})$, $6.81-6.73(\mathrm{~m}, 2 \mathrm{H}), 6.62(\mathrm{~d}, J=8.0 \mathrm{~Hz}, 2 \mathrm{H}), 6.46(\mathrm{~s}, 1 \mathrm{H}), 5.95(\mathrm{~s}, 1 \mathrm{H})$, $5.45(\mathrm{~d}, J=4.8 \mathrm{~Hz}, 1 \mathrm{H}), 4.31(\mathrm{~d}, J=4.3 \mathrm{~Hz}, 1 \mathrm{H}), 3.77(\mathrm{~s}, 3 \mathrm{H}) ;{ }^{13} \mathrm{C} \operatorname{NMR}\left(101 \mathrm{MHz}, \mathrm{CDCl}_{3}\right) \delta=$ 166.1, 163.1 (dd, $J=249.1,12.6 \mathrm{~Hz}), 146.2,144.9$ (t, $J=8.3 \mathrm{~Hz}), 139.4,129.2,127.4,118.4$, 113.5, $110.3(\mathrm{~d}, J=6.9 \mathrm{~Hz}), 110.1(\mathrm{~d}, J=6.9 \mathrm{~Hz}), 103.1(\mathrm{t}, J=25.4 \mathrm{~Hz}), 58.3,52.0$ ppm; HRMS (m/z): $[\mathrm{M}+\mathrm{Na}]^{+}$calcd for $\mathrm{C}_{17} \mathrm{H}_{15} \mathrm{~F}_{2} \mathrm{NO}_{2} \mathrm{Na}^{+}$326.0963, Found 326.0962.

\section{Methyl 2-((2,5-dimethoxyphenyl)(phenylamino)methyl)acrylate (2y)}

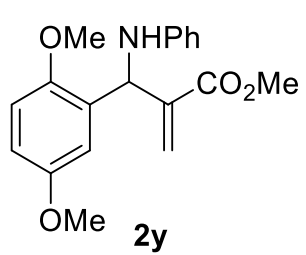

Yellow oil (0.301 g, 92\% yield); $\mathrm{R}_{f}=0.6$ (silica, EtOAc:hexanes, 0.5:9.5);

IR (film) $v_{\max } 3396,3030,1717,1512,819,776 \mathrm{~cm}^{-1} ;{ }^{1} \mathrm{H} \mathrm{NMR}(400 \mathrm{MHz}$, $\left.\mathrm{CDCl}_{3}\right) \delta=7.17-7.13(\mathrm{~m}, 2 \mathrm{H}), 6.89(\mathrm{t}, J=2.5 \mathrm{~Hz}, 1 \mathrm{H}), 6.84(\mathrm{~d}, J=8.8 \mathrm{~Hz}$ 1H), 6.79-6.76 (m, 1H), $6.70(\mathrm{t}, J=7.2 \mathrm{~Hz}, 1 \mathrm{H}), 6.59(\mathrm{~d}, J=7.6 \mathrm{~Hz}, 2 \mathrm{H})$,

$6.35(\mathrm{~s}, 1 \mathrm{H}), 5.84(\mathrm{~s}, 1 \mathrm{H}), 5.80(\mathrm{~s}, 1 \mathrm{H}), 4.26$ (brs, $1 \mathrm{H}), 3.80(\mathrm{~s}, 3 \mathrm{H}), 3.76-3.67(\mathrm{~m}, 6 \mathrm{H}) ;{ }^{13} \mathrm{C}$ $\operatorname{NMR}\left(101 \mathrm{MHz}, \mathrm{CDCl}_{3}\right) \delta=166.8,153.5,151.2,146.8,139.9,129.7,129.0,126.2,117.5$ 114.5, 113.3, 112.6, 112.0, 56.2, 55.6, 52.7, 51.9 ppm; HRMS (m/z): $\left[\mathrm{M}+\mathrm{Na}^{+}\right.$calcd for $\mathrm{C}_{19} \mathrm{H}_{21} \mathrm{NO}_{4} \mathrm{Na}^{+}$350.1362, Found 350.1360.

\section{Methyl 2-((2-fluorophenyl)(phenylamino)methyl)acrylate (2aa)}

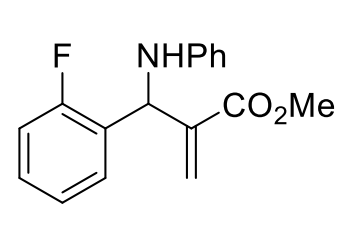

2aa

Yellow oil $\left(0.248 \mathrm{~g}, 87 \%\right.$ yield); $\mathrm{R}_{f}=0.6$ (silica, EtOAc:hexanes, 0.5:9.5);

IR (film) $v_{\max } 3409,3054,1718,1549,1429,1098,1036 \mathrm{~cm}^{-1} ;{ }^{1} \mathrm{H}$ NMR $\left(400 \mathrm{MHz}, \mathrm{CDCl}_{3}\right) \delta=7.36(\mathrm{td}, J=7.6,1.5 \mathrm{~Hz}, 1 \mathrm{H}), 7.36-7.34(\mathrm{~m}, 1 \mathrm{H})$, $7.18-7.05(\mathrm{~m}, 4 \mathrm{H}), 6.73(\mathrm{t}, J=7.3 \mathrm{~Hz}, 1 \mathrm{H}), 6.60(\mathrm{~d}, J=7.7 \mathrm{~Hz}, 2 \mathrm{H}), 6.41$ (s, 1H), $5.91(\mathrm{~s}, 1 \mathrm{H}), 5.76(\mathrm{~d}, J=6.4 \mathrm{~Hz}, 1 \mathrm{H}), 4.26(\mathrm{~d}, J=6.2 \mathrm{~Hz}, 1 \mathrm{H}), 3.73(\mathrm{~s}, 3 \mathrm{H}) ;{ }^{13} \mathrm{C}$ NMR 
$\left(101 \mathrm{MHz} \mathrm{CDCl}_{3}\right) \delta=166.4,160.6(\mathrm{~d}, J=247.8 \mathrm{~Hz}), 146.3,139.3,129.4(\mathrm{~d}, J=8.4 \mathrm{~Hz}), 129.2$, $128.6(\mathrm{~d}, J=3.6 \mathrm{~Hz}), 126.9,124.3(\mathrm{~d}, J=3.5 \mathrm{~Hz}), 118.1,115.7(\mathrm{~d}, J=21.9 \mathrm{~Hz}), 113.4,52.4(\mathrm{~d}$, $J=3.0 \mathrm{~Hz}$ ), 52.0 ppm; HRMS (m/z): $[\mathrm{M}+\mathrm{Na}]^{+}$calcd for $\mathrm{C}_{17} \mathrm{H}_{16} \mathrm{FNO}_{2} \mathrm{Na}^{+}$308.1057, Found 308.1057.

\section{Methyl 2-((4-bromo-3-fluorophenyl)(phenylamino)methyl)acrylate (2ac)}

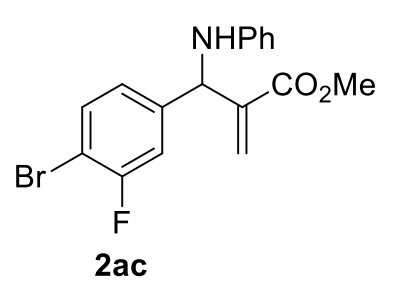

Yellow oil (0.313 g, 86\% yield); $R_{f}=0.6$ (silica, EtOAc:hexanes, 0.5:9.5); IR (film) $v_{\max } 3378,3043,1717,1506,1274,742 \mathrm{~cm}^{-1} ;{ }^{1} \mathrm{H}$ $\operatorname{NMR}\left(400 \mathrm{MHz}, \mathrm{CDCl}_{3}\right) \delta=7.50(\mathrm{t}, J=7.6 \mathrm{~Hz}, 1 \mathrm{H}), 7.18-7.14(\mathrm{~m}$, 3H), $7.07(\mathrm{~d}, J=8.1 \mathrm{~Hz}, 1 \mathrm{H}), 6.75(\mathrm{t}, J=7.3 \mathrm{~Hz}, 1 \mathrm{H}), 6.57(\mathrm{~d}, J=7.8$ $\mathrm{Hz}, 2 \mathrm{H}), 6.41(\mathrm{~s}, 1 \mathrm{H}), 5.93(\mathrm{~s}, 1 \mathrm{H}), 5.38(\mathrm{~s}, 1 \mathrm{H}), 4.23(\mathrm{~s}, 1 \mathrm{H}), 3.72(\mathrm{~s}, 3 \mathrm{H}) ;{ }^{13} \mathrm{C}$ NMR $(101 \mathrm{MHz}$, $\left.\mathrm{CDCl}_{3}\right) \delta=166.1,159.09(\mathrm{~d}, J=248.0 \mathrm{~Hz}), 146.1,142.6(\mathrm{~d}, J=5.9 \mathrm{~Hz}), 139.4,133.6,129.2$, 127.2, $124.2(\mathrm{~d}, J=3.0 \mathrm{~Hz}), 118.3,115.4(\mathrm{~d}, J=23.0 \mathrm{~Hz}), 113.5,108.1$ (d, $J=20.9 \mathrm{~Hz}), 58.2$, $52.1 \mathrm{ppm}$; HRMS (m/z): [M + Na] $]^{+}$calcd for $\mathrm{C}_{17} \mathrm{H}_{15} \mathrm{BrFNO}_{2} \mathrm{Na}^{+}$386.0162, Found 386.0160.

\section{Methyl 2-((perfluorophenyl)(phenylamino)methyl)acrylate (2ad)}

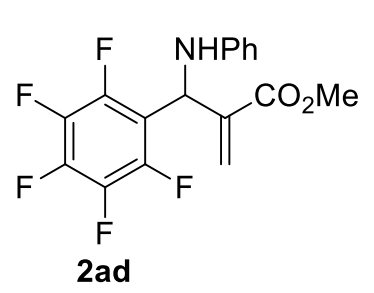

Yellow oil $\left(0.289 \mathrm{~g}, 81 \%\right.$ yield); $\mathrm{R}_{f}=0.6$ (silica, EtOAc:hexanes, 0.5:9.5);

IR (film) $v_{\max } 3400,3019,1714,1437,1101,751 \mathrm{~cm}^{-1} ;{ }^{1} \mathrm{H}$ NMR $(400$ $\left.\mathrm{MHz}, \mathrm{CDCl}_{3}\right) \delta=7.18(\mathrm{t}, J=7.3 \mathrm{~Hz}, 2 \mathrm{H}), 6.77(\mathrm{t}, J=7.3 \mathrm{~Hz}, 1 \mathrm{H}), 6.67$ (d, J = 7.3 Hz, 2H), 6.49 (s, 1H), $6.02(\mathrm{~s}, 1 \mathrm{H}), 5.91$ (d, J = 9.2 Hz, 1H), 4.34 (brs, $1 \mathrm{H}), 3.75(\mathrm{~d}, J=1.7 \mathrm{~Hz}, 3 \mathrm{H}) ;{ }^{13} \mathrm{C} \operatorname{NMR}\left(101 \mathrm{MHz}, \mathrm{CDCl}_{3}\right) \delta=165.8,145.1(\mathrm{~d}, J=$ 246.3 Hz), 145.3, 139.08 (d, $J=63.4 \mathrm{~Hz}), 137.6(\mathrm{~d}, J=245.1 \mathrm{~Hz}), 137.4,129.4,128.9,126.8$, 123.68 (s), 122.3 (d, J = 285.6 Hz), 119.1, 114.4 (d, J = 13.2 Hz), 113.6, 52.1, 49.0 ppm; HRMS (m/z): $[\mathrm{M}+\mathrm{Na}]^{+}$calcd for $\mathrm{C}_{17} \mathrm{H}_{12} \mathrm{~F}_{5} \mathrm{NO}_{2} \mathrm{Na}^{+} 380.0680$, Found 380.0676. 


\section{General procedure for the optimization of the reaction conditions}<smiles></smiles>

To a flame-dried screw-capped test tube equipped with a magnetic stir bar was added the fluoride source $(1.00 \mathrm{mmol})$, and $\mathrm{AMBH}$ adduct $\mathbf{2 a}(0.20 \mathrm{mmol})$ under argon atmosphere. The mixture was dissolved in $4.0 \mathrm{~mL}$ of solvent under argon atmosphere and to this stirring solution was added 2(trimethylsilyl)phenyl trifluoromethanesulfonate $1 \mathrm{a} \quad(0.50 \mathrm{mmol})$ at room temperature. Then the reaction mixture was stirred at rt till the completion of reaction (monitored by TLC). Then the reaction quenched and the solvent was evaporated. The crude residue was purified by flash column chromatography on silica gel.

\section{Optimization studies}

The present study commenced with the treatment of $\mathrm{AMBH}$ adduct $2 \mathrm{a}$ (1.0 equiv) with the aryne generated by fluoride induced 1,2 elimination of 2 -(trimethylsilyl)aryl triflate 1a (1.2 equiv) using 2.5 equiv of $\mathrm{KF}$ in $\mathrm{CH}_{3} \mathrm{CN}$ as the solvent. Under these condition the desired 4-quinolone 6a was indeed obtained in $18 \%$, along with $\mathrm{N}$-arylated amine SI-4a (30\%), fascinatingly, compound $\mathbf{5 a}$ derived from the initial tandem insertion-cyclization product was observed in $41 \%$ and $10 \%$ of 2 a was also recovered (entry 1, Table 1). Intriguingly, when the reaction was carried out using 2.5 equiv of $1 \mathbf{a}$ and 5.0 equiv of KF, 6a was formed in an improved yield of $84 \%$ (entry 4 Table 1 ). Encouraged by these results, several other fluorides such as CsF, TBAF and TBAT were subsequently tested for the reaction. However, other fluorides tested were found to be less effective and in most of the case $2 \mathbf{a}$ was recovered (Table 1, entries 2, 5 and 6). A brief screening of the reaction media proved that acetonitrile was the best choice with respect to 
yields. Moreover ethyl and butyl ester in AMBH adduct also provided desired product albeit with lesser yields and longer reaction time, when compared to methyl ester adduct (entries 7 and 8 , Table 1). Increasing the reaction temperature to $60^{\circ} \mathrm{C}$ did not increase the yield of $6 a$ but $\mathrm{N}$ arylated product SI-4a was formed in $28 \%$ yield (entry 9). Variation in amount of either $\mathbf{1 a}$ or $\mathbf{2 a}$ was not proved to be beneficial, leading to decrease in the desired product $\mathbf{6 a}$. Further, use of bases $\mathrm{DBU}, \mathrm{KO}{ }^{t} \mathrm{Bu}, \mathrm{K}_{2} \mathrm{CO}_{3}$ and $\mathrm{Cs}_{2} \mathrm{CO}_{3}$ as additives didn't improvise the yield of $6 \mathrm{a}$.

Table 1: Optimization of Reaction Condition ${ }^{\mathrm{a}}$

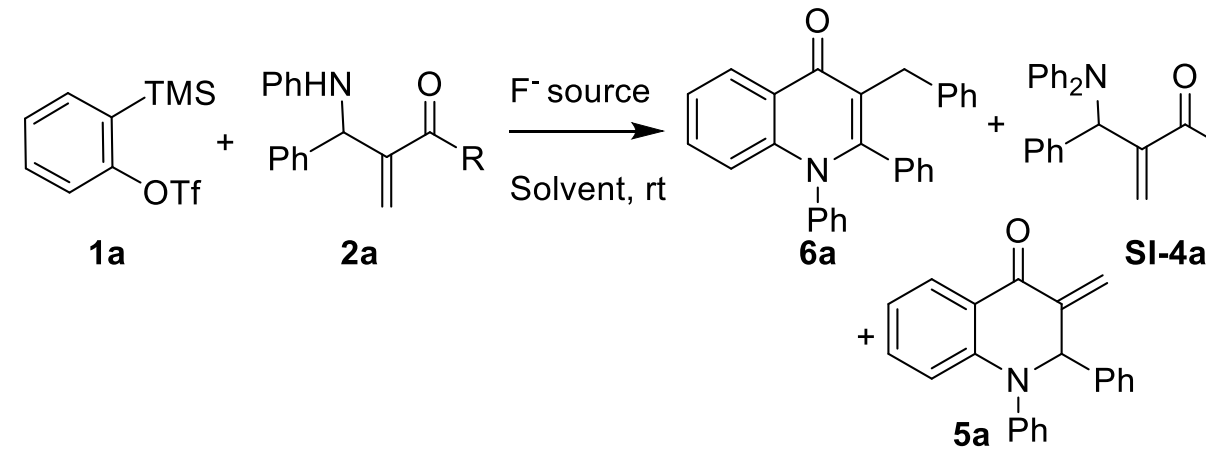

\begin{tabular}{|c|c|c|c|c|c|c|c|c|}
\hline \multirow[b]{2}{*}{ entry } & \multirow[b]{2}{*}{$F^{-}$source } & & \multirow{2}{*}{ Time } & \multicolumn{3}{|c|}{ Yield $(\%)^{b}$} \\
\hline & & $\mathrm{R}$ & (equiv) & solvent & & $6 a$ & SI-4ab & $5 a$ \\
\hline 1 & $\mathrm{KF}^{\mathrm{d}}$ & $\mathrm{Me}$ & 1.2 & $\mathrm{CH}_{3} \mathrm{CN}$ & 4 & 14 & 20 & $49(10)^{\mathrm{C}}$ \\
\hline 2 & $\mathrm{CsF}^{\mathrm{e}}$ & $\mathrm{Me}$ & 1.2 & $\mathrm{CH}_{3} \mathrm{CN}$ & 9 & - & 68 & $-(20)^{c}$ \\
\hline 3 & $\mathrm{KF}^{\mathrm{f}}$ & $\mathrm{Me}$ & 1.2 & THF & 5 & 54 & 25 & $-(12)^{c}$ \\
\hline 4 & $\mathrm{KF}^{\mathrm{f}}$ & Me & 2.5 & $\mathrm{CH}_{3} \mathrm{CN}$ & 4 & 84 & 8 & \\
\hline 5 & TBAF $^{\mathrm{e}}$ & $\mathrm{Me}$ & 2.5 & $\mathrm{CH}_{3} \mathrm{CN}$ & 24 & - & 10 & $12(45)^{\mathrm{c}}$ \\
\hline 6 & TBAT $^{\mathrm{e}}$ & $\mathrm{Me}$ & 2.5 & $\mathrm{CH}_{3} \mathrm{C}$ & 24 & 14 & 32 & $22(28)^{\mathrm{C}}$ \\
\hline 7 & $\mathrm{KF}$ & Et & 2.5 & $\mathrm{CH}_{3} \mathrm{CN}$ & 12 & 72 & 12 & - \\
\hline 8 & $\mathrm{KF}$ & ${ }^{\mathrm{B}} \mathrm{Bu}$ & 2.5 & $\mathrm{CH}_{3} \mathrm{CN}$ & 14 & 70 & 12 & - \\
\hline & $\mathrm{KF}$ & $\mathrm{Me}$ & 2.5 & $\mathrm{CH}_{3} \mathrm{CN}$ & 4 & 64 & 28 & \\
\hline \multicolumn{9}{|c|}{$\begin{array}{l}\text { a Standard conditions: } 1 \mathrm{a}(0.20 \mathrm{mmol}), 2 \mathrm{a}(0.50 \mathrm{mmol}) \text {, fluoride source }(1.00 \\
\text { mmol), solvent }(4.0 \mathrm{~mL}) \text {, rt, } 4 \mathrm{~h} \text {. }{ }^{b} \text { Isolated yields. }{ }^{\circ} \text { Recovery yield of } 2 \mathrm{a} \text {. }{ }^{\mathrm{d}} 2.5 \\
\text { equiv of } \mathrm{F}^{-}, 2.5 \text { equiv of } 18 \text {-crown- } 6^{\circ} 5.0 \text { equiv of } \mathrm{F}^{-} .{ }^{\dagger} 5.0 \text { equiv of } \mathrm{F}^{-}, 5.0 \text { equiv } \\
\text { of } 18 \text {-crown-6. }{ }^{9} \text { reaction temperature } 60^{\circ} \mathrm{C}\end{array}$} \\
\hline
\end{tabular}

\section{3-Methylene-1,2-diphenyl-2,3-dihydroquinolin-4(1H)-one (5a)}<smiles>C=C1C(=O)c2ccccc2N(c2ccccc2)C1c1ccccc1</smiles>

$5 a$

Yellow solid (0.030 g, 49\% yield, m.p. $109.5-111.2{ }^{\circ} \mathrm{C}$ ); $\mathrm{R}_{f}=0.5$ (silica, EtOAc:hexanes, 0.5:9.5); IR (film) $v_{\max } 3019,2999,1720,1428,1410,982 \mathrm{~cm}^{-}$ '; ${ }^{1} \mathrm{H}$ NMR $\left(400 \mathrm{MHz}, \mathrm{CDCl}_{3}\right) \delta=7.91(\mathrm{~d}, J=7.9 \mathrm{~Hz}, 1 \mathrm{H}), 7.33-7.20(\mathrm{~m}$, 
$10 \mathrm{H}), 7.14$ (t, $J=7.3 \mathrm{~Hz}, 1 \mathrm{H}), 6.99(\mathrm{~d}, J=8.5 \mathrm{~Hz}, 1 \mathrm{H}), 6.79(\mathrm{t}, J=7.5 \mathrm{~Hz}, 1 \mathrm{H}), 6.30(\mathrm{~s}, 1 \mathrm{H})$, $5.61(\mathrm{~s}, 1 \mathrm{H}), 5.52(\mathrm{~s}, 1 \mathrm{H}) ;{ }^{13} \mathrm{C} \operatorname{NMR}\left(101 \mathrm{MHz} \mathrm{CDCl}_{3}\right) \delta=182.9,148.9,145.5,143.2,139.4$, 135.2, 129.6, 128.7, 127.5, 126.7, 124.9, 123.6, 122.8, 121.6, 119.4, 117.4, 69.4 ppm; MS $\left(\mathrm{ES}^{+}\right): \mathrm{m} / \mathrm{z} 312.4\left[\mathrm{M}+\mathrm{H}^{+}\right]$.

\section{Methyl 2-((diphenylamino)(phenyl)methyl)acrylate (SI-4a)}<smiles>C=C(C(=O)OC)C(c1ccccc1)c1ccccc1</smiles>

SI-4a

Yellow oil ( $0.046 \mathrm{~g}, 68 \%$ yield); $\mathrm{R}_{f}=0.5$ (silica, EtOAc:hexanes, 0.5:9.5);

IR (film) $V_{\max } 3014,2999,1712,1640,1482,1015,985 \mathrm{~cm}^{-1} ;{ }^{1} \mathrm{H}$ NMR $\left(400 \mathrm{MHz}, \mathrm{CDCl}_{3}\right) \delta=7.25(\mathrm{~d}, J=6.0 \mathrm{~Hz}, 2 \mathrm{H}), 7.23-7.17(\mathrm{~m}, 2 \mathrm{H}), 7.17$ - $7.10(\mathrm{~m}, 5 \mathrm{H}), 6.89(\mathrm{t}, J=7.7 \mathrm{~Hz}, 6 \mathrm{H}), 6.36(\mathrm{~d}, J=1.1 \mathrm{~Hz}, 1 \mathrm{H}), 6.34(\mathrm{~s}, 1 \mathrm{H}), 5.75(\mathrm{t}, J=1.3 \mathrm{~Hz}$, 1H), $3.64(\mathrm{~s}, 3 \mathrm{H}) ;{ }^{13} \mathrm{C}$ NMR $\left(101 \mathrm{MHz}, \mathrm{CDCl}_{3}\right) \delta=166.9,146.5,140.4,138.5,129.2,128.7$, 128.2, 128.1, 127.2, 123.3, 121.7, 64.1, 51.9 ppm; MS $\left(\mathrm{ES}^{+}\right): \mathrm{m} / z 344.4\left[\mathrm{M}+\mathrm{H}^{+}\right]$.

\section{Synthesis and characterization of 4-quinolones via ene reaction}<smiles>C=C1C(=O)c2ccccc2N(c2ccccc2)C1c1ccccc1</smiles>

$5 a$

(1 equiv)<smiles>[R]c1ccc([SiH3])c(O[GaH])c1</smiles>

1

(1.2 equiv)

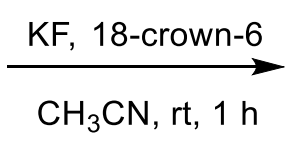

$\mathrm{CH}_{3} \mathrm{CN}$, rt, $1 \mathrm{~h}$

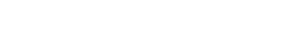<smiles>[R][R]1ccc(Cc2c(-c3ccccc3)n(-c3ccccc3)c3ccccc3c2=O)cc1</smiles>

6a-g

General Procedure: To a flame-dried screw-capped test tube equipped with a magnetic stir bar was added the 18-crown-6 (95.0 mg, $0.36 \mathrm{mmol})$ and $\mathrm{KF}(20.9 \mathrm{mg}, 0.36 \mathrm{mmol})$ under argon atmosphere, then enone $5 \mathrm{a}(0.15 \mathrm{mmol})$ was added and the screw-capped tube was evacuated and backfilled with argon. The mixture was dissolved in $\mathrm{CH}_{3} \mathrm{CN}$ (3.0 mL) under argon atmosphere. The resultant reaction mixture was kept stirring at room temperature for five minutes. To the stirring solution was added $\beta$-trimethylsilyltriflate $1(0.18 \mathrm{mmol})$ dropwise, further allowed to stir for $1 \mathrm{~h}$ (12 hours for $\mathbf{6 g}$ ). Then the reaction quenched and the solvent was 
evaporated. Subsequently, the crude residue was purified by flash column chromatography on silica gel to afford the corresponding 4-quinolone derivatives 6a-g in high yields.

\section{3-Benzyl-1,2-diphenylquinolin-4(1H)-one (6a)}<smiles>O=c1c(Cc2ccccc2)c(-c2ccccc2)n(-c2ccccc2)c2ccccc12</smiles>

White solid (0.065 g, 84\% yield, m.p. 200.5-201.2 ${ }^{\circ} \mathrm{C}$ ); $\mathrm{R}_{f}=0.4$ (silica, EtOAc:hexanes, 1:4); IR (film) $v_{\max } 2999,1635,1580,1494,1478,698$ $\mathrm{cm}^{-1} ;{ }^{1} \mathrm{H}$ NMR $\left(400 \mathrm{MHz}, \mathrm{CDCl}_{3}\right) \delta=8.58(\mathrm{~d}, J=8.0 \mathrm{~Hz}, 1 \mathrm{H}), 7.45-$ $7.40(\mathrm{~m}, 1 \mathrm{H}), 7.38-7.34(\mathrm{~m}, 1 \mathrm{H}), 7.31-7.21(\mathrm{~m}, 3 \mathrm{H}), 7.17-7.03(\mathrm{~m}$,

$8 \mathrm{H}), 6.99(\mathrm{~d}, J=7.4 \mathrm{~Hz}, 2 \mathrm{H}), 6.94-6.92(\mathrm{~m}, 2 \mathrm{H}), 6.74(\mathrm{~d}, J=8.5 \mathrm{~Hz}, 1 \mathrm{H}), 3.80(\mathrm{~s}, 2 \mathrm{H}) ;{ }^{13} \mathrm{C}$ $\operatorname{NMR}\left(101 \mathrm{MHz}, \mathrm{CDCl}_{3}\right) \delta=177.4,151.9,142.0,141.5,139.6,134.2,131.5,130.1,129.7$, 129.3, 128.6, 128.5, 128.2, 127.8, 127.7, 126.6, 125.3, 125.1, 123.4, 121.4, 117.9, 32.4 ppm; HRMS (m/z): $[\mathrm{M}+\mathrm{Na}]^{+}$calcd for $\mathrm{C}_{28} \mathrm{H}_{21} \mathrm{NONa}^{+} 410.1515$, Found 410.1502 .

\section{3-(Benzo[d][1,3]dioxol-5-ylmethyl)-1,2-diphenylquinolin-4(1H)-one (6b)}<smiles>O=c1c(Cc2ccc3c(c2)OCO3)c(-c2ccccc2)n(-c2ccccc2)c2ccccc12</smiles>

6b
White solid $\left(0.057 \mathrm{~g}, 89 \%\right.$ yield, m.p. $\left.143.0-146.2{ }^{\circ} \mathrm{C}\right) ; \mathrm{R}_{f}=0.5$ (silica, EtOAc:hexanes, 1:3); IR (film) $\mathrm{v}_{\max } 3012,1618,1592$, $1496,1474,765 \mathrm{~cm}^{-1} ;{ }^{1} \mathrm{H} \mathrm{NMR}\left(400 \mathrm{MHz}, \mathrm{CDCl}_{3}\right) \delta=8.56(\mathrm{dd}$, $J=8.1,1.7 \mathrm{~Hz}, 1 \mathrm{H}), 7.48-7.31(\mathrm{~m}, 2 \mathrm{H}), 7.31-7.19(\mathrm{~m}, 3 \mathrm{H})$,

$7.15(\mathrm{dd}, J=5.1,1.9 \mathrm{~Hz}, 3 \mathrm{H}), 7.10-6.92(\mathrm{~m}, 4 \mathrm{H}), 6.74(\mathrm{~d}, J=8.5 \mathrm{~Hz}, 1 \mathrm{H}), 6.61-6.52(\mathrm{~m}, 2 \mathrm{H})$, $6.38(\mathrm{dd}, J=8.0,1.7 \mathrm{~Hz}, 1 \mathrm{H}), 5.83(\mathrm{~s}, 2 \mathrm{H}), 3.71(\mathrm{~s}, 2 \mathrm{H}) ;{ }^{13} \mathrm{C} \mathrm{NMR}\left(101 \mathrm{MHz}, \mathrm{CDCl}_{3}\right) \delta=177.4$, 151.9, 147.0, 145.1, 141.9, 139.5, 135.2, 134.1, 131.5, 130.1, 129.6, 129.3, 128.6, 128.2, 127.7, 126.5, 125.0, 123.4, 121.3, 121.2, 117.9, 109.1, 107.5, 100.4, 32.1 ppm; HRMS (m/z): [M + Na ${ }^{+}$ calcd for $\mathrm{C}_{29} \mathrm{H}_{21} \mathrm{NO}_{3} \mathrm{Na}^{+}$454.1413, Found 454.1397.

\section{3-(3,4-diMethoxybenzyl)-1,2-diphenylquinolin-4(1H)-one (6c)}




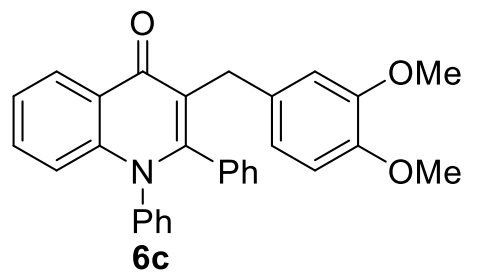

Colorless oil $\left(0.062 \mathrm{~g}, \quad 92 \%\right.$ yield); $\mathrm{R}_{f}=0.4$ (silica, EtOAc:hexanes, 1:3); IR (film) $v_{\max } 3008,1624,1588,1492,1478$, $790 \mathrm{~cm}^{-1} ;{ }^{1} \mathrm{H}$ NMR $\left(400 \mathrm{MHz}, \mathrm{CDCl}_{3}\right) \delta=8.57(\mathrm{dd}, J=8.0,1.7$ $\mathrm{Hz}, 1 \mathrm{H}), 7.44-7.33(\mathrm{~m}, 2 \mathrm{H}), 7.31-7.20(\mathrm{~m}, 3 \mathrm{H}), 7.15(\mathrm{dd}, J=$ $5.1,2.0 \mathrm{~Hz}, 3 \mathrm{H}), 7.09-7.02(\mathrm{~m}, 2 \mathrm{H}), 6.99-6.92(\mathrm{~m}, 2 \mathrm{H}), 6.76-6.70(\mathrm{~m}, 1 \mathrm{H}), 6.63(\mathrm{~d}, J=8.2$ $\mathrm{Hz}, 1 \mathrm{H}), 6.60-6.50(\mathrm{~m}, 2 \mathrm{H}), 3.79(\mathrm{~s}, 3 \mathrm{H}), 3.75(\mathrm{~s}, 2 \mathrm{H}), 3.73(\mathrm{~s}, 3 \mathrm{H}) ;{ }^{13} \mathrm{C}$ NMR $(101 \mathrm{MHz}$, $\left.\mathrm{CDCl}_{3}\right) \delta=177.4,151.7,148.1,146.7,141.9,139.5,134.3,134.2,131.4,130.1,129.8,129.3$ 128.6, 128.1, 127.7, 126.5, 125.0, 123.3, 121.7, 120.4, 117.92, 112.2, 110.7, 55.7, 55.6, 31.9 ppm; HRMS (m/z): [M + Na] $]^{+}$calcd for $\mathrm{C}_{30} \mathrm{H}_{25} \mathrm{NO}_{3} \mathrm{Na}^{+} 470.1726$, Found 470.1714 .

\section{3-((2,3-diHydro-1H-inden-5-yl)methyl)-1,2-diphenylquinolin-4(1H)-one (6d)}

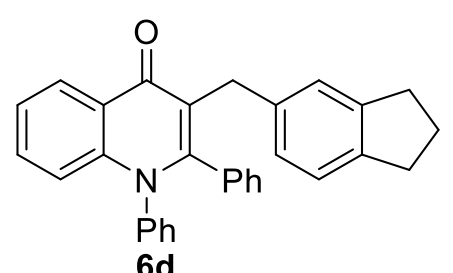

6d
Colorless oil $\left(0.059 \mathrm{~g}, 91 \%\right.$ yield); $\mathrm{R}_{f}=0.5$ (silica, EtOAc:hexanes, 1:4); IR (film) $v_{\max } 3010,1630,1582,1496,1474$, $910 \mathrm{~cm}^{-1} ;{ }^{1} \mathrm{H}$ NMR $\left(400 \mathrm{MHz}, \mathrm{CDCl}_{3}\right) \delta=8.57(\mathrm{dd}, J=7.9,1.7$ $\mathrm{Hz}, 1 \mathrm{H}), 7.47-7.39(\mathrm{~m}, 1 \mathrm{H}), 7.35(\mathrm{t}, J=7.5 \mathrm{~Hz}, 1 \mathrm{H}), 7.30-7.23$

(m, 4H), $7.18-7.11(\mathrm{~m}, 2 \mathrm{H}), 7.11-7.05(\mathrm{~m}, 2 \mathrm{H}), 7.01-6.92(\mathrm{~m}, 3 \mathrm{H}), 6.89(\mathrm{~s}, 1 \mathrm{H}), 6.74(\mathrm{dd}, J$ $=8.4,2.5 \mathrm{~Hz}, 2 \mathrm{H}), 3.76(\mathrm{~s}, 2 \mathrm{H}), 2.82-2.75(\mathrm{~m}, 4 \mathrm{H}), 2.02-1.95(\mathrm{~m}, 2 \mathrm{H}) ;{ }^{13} \mathrm{C} N M R(101 \mathrm{MHz}$, $\left.\mathrm{CDCl}_{3}\right) \delta=177.4,151.7,143.8,141.9,141.1,139.7,139.2,134.3,131.4,130.2,129.8,129.4$ 129.3, 128.5, 128.1, 127.7, 126.6, 126.3, 125.1, 124.6, 123.6, 123.3, 121.8, 117.8, 32.8, 32.4, 32.3, 25.4 ppm; HRMS (m/z): [M + Na] ${ }^{+}$calcd for $\mathrm{C}_{31} \mathrm{H}_{25} \mathrm{NONa}^{+}$450.1828, Found 450.1811.

\section{3-(3,4-diMethylbenzyl)-1,2-diphenylquinolin-4(1H)-one (6e)}<smiles>Cc1ccc(Cc2c(-c3ccccc3)n(-c3ccccc3)c3ccccc3c2=O)cc1C</smiles>

White solid (0.058 g, 92\% yield, m.p. $\left.207.1-209.3{ }^{\circ} \mathrm{C}\right) ; \mathrm{R}_{f}=0.5$ (silica, EtOAc:hexanes, 1:4); IR (film) $v_{\max } 3025,2290,1618,1495$, $1478,890 \mathrm{~cm}^{-1} ;{ }^{1} \mathrm{H}$ NMR $\left(400 \mathrm{MHz}, \mathrm{CDCl}_{3}\right) \delta=8.57(\mathrm{dd}, J=8.0$, $1.7 \mathrm{~Hz}, 1 \mathrm{H}), 7.44-7.32(\mathrm{~m}, 2 \mathrm{H}), 7.31-7.20(\mathrm{~m}, 3 \mathrm{H}), 7.20-7.10$ 
(m, 3H), $7.10-7.03(\mathrm{~m}, 2 \mathrm{H}), 7.00-6.93(\mathrm{~m}, 2 \mathrm{H}), 6.88(\mathrm{~d}, J=7.6 \mathrm{~Hz}, 1 \mathrm{H}), 6.78-6.69(\mathrm{~m}, 3 \mathrm{H})$, $3.73(\mathrm{~s}, 2 \mathrm{H}), 2.15(\mathrm{~s}, 3 \mathrm{H}), 2.11(\mathrm{~s}, 3 \mathrm{H}) ;{ }^{13} \mathrm{C} \mathrm{NMR}\left(101 \mathrm{MHz}, \mathrm{CDCl}_{3}\right) \delta=177.4,151.7,141.9$, 139.6, 138.7, 135.7, 134.2, 133.3, 131.4, 130.1, 129.9, 129.8, 129.3, 129.0, 128.5, 128.1, 127.7 , 126.6, 125.8, 125.1, 123.2, 121.7, 117.8, 32.0, 19.6, 19.2 ppm; HRMS (m/z): [M + Na] $]^{+}$calcd for $\mathrm{C}_{30} \mathrm{H}_{25} \mathrm{NONa}^{+}$438.1828, Found 438.1818.

\section{3-(Naphthalen-2-ylmethyl)-1,2-diphenylquinolin-4(1H)-one (6f)}

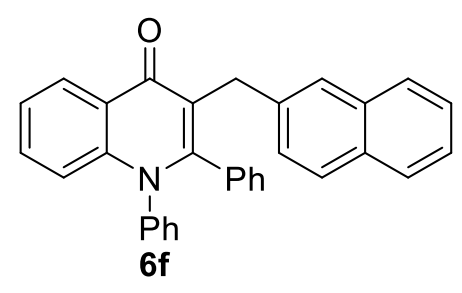

White solid (0.060 g, 91\% yield, m.p. 204.5-206.6 $\left.{ }^{\circ} \mathrm{C}\right) ; \mathrm{R}_{f}=0.5$ (silica, EtOAc:hexanes, 1:4); IR (film) $v_{\max } 3008,1618,1521,1495$, 1478, 1405, $890 \mathrm{~cm}^{-1} ;{ }^{1} \mathrm{H}$ NMR $\left(400 \mathrm{MHz} \mathrm{CDCl}_{3}\right) \delta=8.63(\mathrm{dd}, J=$ 7.9, $1.8 \mathrm{~Hz}, 1 \mathrm{H}), 7.76-7.68(\mathrm{~m}, 1 \mathrm{H}), 7.61(\mathrm{t}, J=7.5 \mathrm{~Hz}, 2 \mathrm{H}), 7.48$ - $7.32(\mathrm{~m}, 4 \mathrm{H}), 7.31-7.20(\mathrm{~m}, 4 \mathrm{H}), 7.20-7.14(\mathrm{~m}, 2 \mathrm{H}), 7.13-7.02(\mathrm{~m}, 4 \mathrm{H}), 6.93-6.86(\mathrm{~m}$, 2H), $6.76(\mathrm{~d}, J=8.4 \mathrm{~Hz}, 1 \mathrm{H}), 3.98(\mathrm{~s}, 2 \mathrm{H}) ;{ }^{13} \mathrm{C}$ NMR $\left(101 \mathrm{MHz}, \mathrm{CDCl}_{3}\right) \delta=177.4,152.2,141.9$, $139.5,139.0,134.1,133.3,131.8,131.5,130.0,129.7,129.3,128.6,128.2,127.7,127.7,127.4$, 127.3, 127.3, 126.6, 126.4, 125.3, 125.1, 124.7, 123.4, 121.2, 117.9, 32.5 ppm; HRMS (m/z): [M $+\mathrm{Na}]^{+}$calcd for $\mathrm{C}_{32} \mathrm{H}_{23} \mathrm{NONa}^{+} 460.1671$, Found 460.1654 .

\section{3-(3,4-diFluorobenzyl)-1,2-diphenylquinolin-4(1H)-one (6g)}

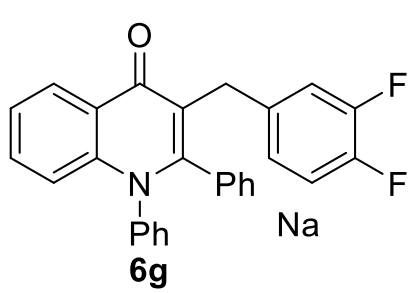

Gum (0.048 g, 75\% yield); $R_{f}=0.5$ (silica, EtOAc:hexanes, 1:4); IR (film) $v_{\max } 3010,1616,1540,1478,1180,780 \mathrm{~cm}^{-1} ;{ }^{1} \mathrm{H}$ NMR $(400$ $\left.\mathrm{MHz}, \mathrm{CDCl}_{3}\right) \delta=8.55(\mathrm{dd}, J=8.1,1.5 \mathrm{~Hz}, 1 \mathrm{H}), 7.49-7.34(\mathrm{~m}, 2 \mathrm{H})$, $7.32-7.24(m, 3 H), 7.16(d, J=6.6 \mathrm{~Hz}, 3 \mathrm{H}), 7.11-7.05(\mathrm{~m}, 2 \mathrm{H}), 6.96$ - $6.83(\mathrm{~m}, 3 \mathrm{H}), 6.77(\mathrm{t}, J=7.4 \mathrm{~Hz}, 2 \mathrm{H}), 6.67(\mathrm{t}, J=6.3 \mathrm{~Hz}, 1 \mathrm{H}), 3.73(\mathrm{~s}, 2 \mathrm{H}) ;{ }^{13} \mathrm{C}$ NMR $(101$ $\left.\mathrm{MHz}_{\mathrm{CDCl}}\right) \delta=177.3,152.2,150.9(\mathrm{~d}, J=12 \mathrm{~Hz}), 149.7(\mathrm{~d}, J=12 \mathrm{~Hz}), 148.5(\mathrm{~d}, J=12 \mathrm{~Hz})$, $147.3(\mathrm{~d}, \mathrm{~J}=12 \mathrm{~Hz}), 146.2,141.9,139.4,138.3,134.6,133.9,131.7,130.0,129.4,129.4$, $128.7,128.4,127.9,126.5,125.0,124.2,123.7,120.6,118.0,117.1(d, J=17 \mathrm{~Hz}), 116.3(d, J=$ 
$12 \mathrm{~Hz}), 115.2,109.5(\mathrm{~d}, J=12 \mathrm{~Hz}), 31.7 \mathrm{ppm} ; \mathrm{HRMS}(\mathrm{m} / \mathrm{z}):[\mathrm{M}+\mathrm{Na}]^{+}$calcd for $\mathrm{C}_{28} \mathrm{H}_{19} \mathrm{~F}_{2} \mathrm{NONa}^{+}$ 446.1332, Found 446.1326.

\section{Synthesis and characterization of 4-quinolones via cascade reaction}

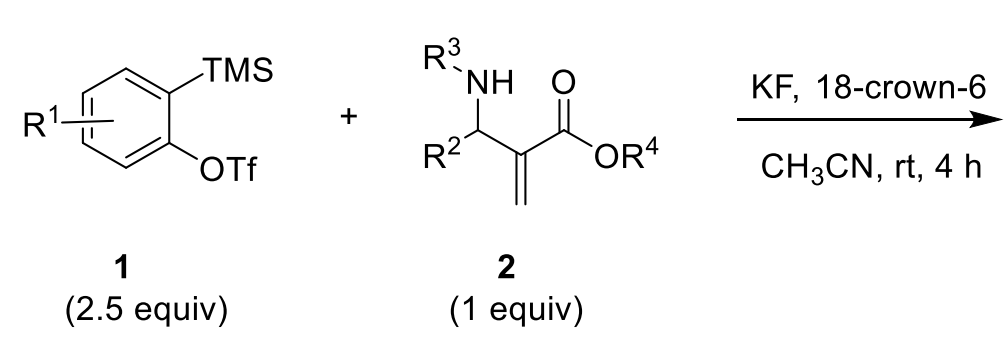

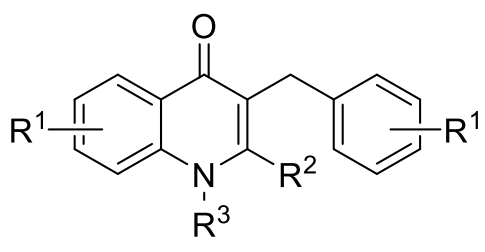

6

General Procedure: To a flame-dried screw-capped test tube equipped with a magnetic stir bar was added the 18-crown-6 (263 mg, $1.00 \mathrm{mmol}, 5.0$ equiv) and $\mathrm{KF}$ (59 mg, $1.00 \mathrm{mmol}, 5.0$ equiv) under argon atmosphere, then AMBH adduct 2 (0.20 mmol, 1.0 equiv) was added and the screw-capped tube was evacuated and backfilled with argon. The mixture was dissolved in $\mathrm{CH}_{3} \mathrm{CN}(3.0 \mathrm{~mL})$ under argon atmosphere. The resultant reaction mixture was kept stirring at room temperature for five minutes. To the stirring solution was added $\beta$-trimethylsilyltriflate 1 ( $0.5 \mathrm{mmol}, 2.5$ equiv) dropwise, further allowed to stir for $4 \mathrm{~h}$ (12 hours for 6 an). Then the reaction quenched and the solvent was evaporated. Subsequently, the crude residue was purified by flash column chromatography on silica gel to afford the corresponding 4-quinolone derivatives $6 \mathrm{~h}-6 \mathrm{ao}$ in high yields.

\section{3-Benzyl-1-(3-bromophenyl)-2-phenylquinolin-4(1H)-one (6h)}<smiles>O=c1c(Cc2ccccc2)c(-c2ccccc2)n(-c2cccc(Br)c2)c2ccccc12</smiles>

6h

White solid (0.073 g, 73\% yield, m.p. 184.1-185.1 ${ }^{\circ} \mathrm{C}$ ); $\mathrm{R}_{f}=0.5$ (silica, EtOAc:hexanes, 1:4); IR (film) $v_{\max } 3009,1618,1515,1478,1090,768 \mathrm{~cm}^{-}$ 1; ${ }^{1} \mathrm{H}$ NMR $\left(400 \mathrm{MHz}, \mathrm{CDCl}_{3}\right) \delta=8.57(\mathrm{dd}, J=8.1,1.7 \mathrm{~Hz}, 1 \mathrm{H}), 7.49-7.43$ $(\mathrm{m}, 1 \mathrm{H}), 7.42-7.31(\mathrm{~m}, 3 \mathrm{H}), 7.26(\mathrm{~d}, J=2.3 \mathrm{~Hz}, 1 \mathrm{H}), 7.23-7.07(\mathrm{~m}, 6 \mathrm{H})$, $7.07-7.00(m, 1 H), 7.00-6.90(m, 4 H), 6.74(d, J=8.5 H z, 1 H), 3.88-$ $3.71(\mathrm{~m}, 2 \mathrm{H}) ;{ }^{13} \mathrm{C}$ NMR $\left(101 \mathrm{MHz}, \mathrm{CDCl}_{3}\right) \delta=177.4,151.4,141.6,141.2,140.7,133.8,133.4$, 
$131.8,131.7,130.4,129.6,129.5,128.9,128.5,128.5,128.0,127.9,127.8,126.7,125.4,125.0$ 123.5, 122.6, 121.6, 117.6, $32.4 \mathrm{ppm}$; $\operatorname{HRMS}(\mathrm{m} / \mathrm{z}):[\mathrm{M}+\mathrm{Na}]^{+}$calcd for $\mathrm{C}_{28} \mathrm{H}_{20} \mathrm{BrNONa}^{+}$ 488.0620, Found 488.0605 .

\section{3-Benzyl-1-(4-bromophenyl)-2-phenylquinolin-4(1H)-one (6i)}

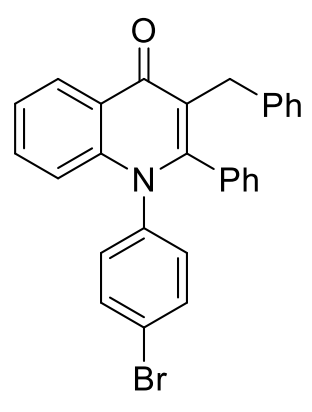

$6 \mathbf{i}$

White solid (0.071 g, 76\% yield, m.p. $261.8-262.7{ }^{\circ} \mathrm{C}$ ); $\mathrm{R}_{f}=0.5$ (silica, EtOAc:hexanes, 1:4); IR (film) $v_{\max } 3030,1615,1530,1472,1200,745 \mathrm{~cm}^{-}$ 1; ${ }^{1} \mathrm{H}$ NMR $\left(400 \mathrm{MHz}, \mathrm{CDCl}_{3}\right) \delta=8.57(\mathrm{dd}, J=8.0,1.7 \mathrm{~Hz}, 1 \mathrm{H}), 7.49-7.33$ $(\mathrm{m}, 4 \mathrm{H}), 7.25-7.14(\mathrm{~m}, 3 \mathrm{H}), 7.13-7.01(\mathrm{~m}, 3 \mathrm{H}), 7.01-6.88(\mathrm{~m}, 6 \mathrm{H}), 6.71$ $(\mathrm{d}, J=8.5 \mathrm{~Hz}, 1 \mathrm{H}), 3.78(\mathrm{~s}, 2 \mathrm{H}) ;{ }^{13} \mathrm{C}$ NMR $\left(101 \mathrm{MHz}, \mathrm{CDCl}_{3}\right) \delta=177.4$, $151.5,141.7,141.3,138.7,133.9,132.6,131.8,131.7,129.5,128.6,128.5$, 128.0, 127.8, 126.7, 125.4, 125.0, 123.6, 122.6, 121.7, 117.6, 32.4 ppm; HRMS (m/z): [M + Na] ${ }^{+}$ calcd for $\mathrm{C}_{28} \mathrm{H}_{20} \mathrm{BrNONa}^{+}$488.0620, Found 488.0606.

\section{3-Benzyl-1-(4-ethylphenyl)-2-phenylquinolin-4(1H)-one (6j)}<smiles>CCc1ccc(-n2c(-c3ccccc3)c(Cc3ccccc3)c(=O)c3ccccc32)cc1</smiles>

6j

White solid (0.066 g, 80\% yield, m.p. 195.2-197.6 ${ }^{\circ} \mathrm{C}$ ); $\mathrm{R}_{f}=0.5$ (silica, EtOAc:hexanes, 1:4); IR (film) $v_{\max } 3015,1618,1530,1478,925,760 \mathrm{~cm}^{-1}$; ${ }^{1} \mathrm{H}$ NMR $\left(400 \mathrm{MHz}, \mathrm{CDCl}_{3}\right) \delta=8.61-8.53(\mathrm{~m}, 1 \mathrm{H}), 7.47-7.31(\mathrm{~m}, 2 \mathrm{H})$, $7.18-7.02(\mathrm{~m}, 8 \mathrm{H}), 7.02-6.87(\mathrm{~m}, 6 \mathrm{H}), 6.77(\mathrm{~d}, J=8.6 \mathrm{~Hz}, 1 \mathrm{H}), 3.80(\mathrm{~s}$, 2H), 2.57 (q, $J=7.6 \mathrm{~Hz}, 2 \mathrm{H}), 1.16(\mathrm{t}, J=7.6 \mathrm{~Hz}, 3 \mathrm{H}) ;{ }^{13} \mathrm{C} \mathrm{NMR}(101 \mathrm{MHz}$, $\left.\mathrm{CDCl}_{3}\right) \delta=177.4,152.1,144.7,142.1,141.5,137.1,134.3,131.4,129.8$ 129.6, 128.6, 128.5, 128.0, 127.7, 127.7, 126.5, 125.3, 125.1, 123.3, 121.3, 118.0, 32.5, 28.3, $15.1 \mathrm{ppm} ; \mathrm{HRMS}(\mathrm{m} / \mathrm{z}):[\mathrm{M}+\mathrm{Na}]^{+}$calcd for $\mathrm{C}_{30} \mathrm{H}_{25} \mathrm{NONa}^{+}$438.1828, Found 438.1813.

\section{3-Benzyl-2-(4-chlorophenyl)-1-phenylquinolin-4(1H)-one (6k)}<smiles>O=c1c(Cc2ccccc2)c(-c2ccc(Cl)cc2)n(-c2ccccc2)c2ccccc12</smiles>

$6 k$
White solid (0.066 g, 78\% yield, m.p. $234.7-236.0^{\circ} \mathrm{C}$ ); $\mathrm{R}_{f}=0.5$ (silica, EtOAc:hexanes, 1:4); IR (film) $v_{\max } 2999,1615,1528,1472,1126,780$ 
$\mathrm{cm}^{-1} ;{ }^{1} \mathrm{H}$ NMR $\left(400 \mathrm{MHz}, \mathrm{CDCl}_{3}\right) \delta=8.57(\mathrm{dd}, J=8.0,1.7 \mathrm{~Hz}, 1 \mathrm{H}), 7.48-7.33(\mathrm{~m}, 2 \mathrm{H}), 7.33-$ $7.26(\mathrm{~m}, 3 \mathrm{H}), 7.09$ (ddd, $J=14.9,7.7,4.6 \mathrm{~Hz}, 7 \mathrm{H}), 7.01-6.95(\mathrm{~m}, 2 \mathrm{H}), 6.85(\mathrm{~d}, J=8.2 \mathrm{~Hz}, 2 \mathrm{H})$, $6.73(\mathrm{~d}, J=8.5 \mathrm{~Hz}, 1 \mathrm{H}), 3.78(\mathrm{~s}, 2 \mathrm{H}) ;{ }^{13} \mathrm{C} \mathrm{NMR}\left(101 \mathrm{MHz}, \mathrm{CDCl}_{3}\right) \delta=177.3,150.6,141.9$, $141.2,139.4,134.3,132.6,131.6,131.0,130.0,129.5,128.8,128.4,128.0,127.9,126.6,125.4$, 125.0, 123.5, 121.6, 117.9, 32.3 ppm; HRMS $(\mathrm{m} / \mathrm{z}):[\mathrm{M}+\mathrm{Na}]^{+}$calcd for $\mathrm{C}_{28} \mathrm{H}_{20} \mathrm{CINONa}^{+}$ 444.1125, Found 444.1112.

\section{3-Benzyl-2-(4-chlorophenyl)-1-(p-tolyl)quinolin-4(1H)-one (6I)}

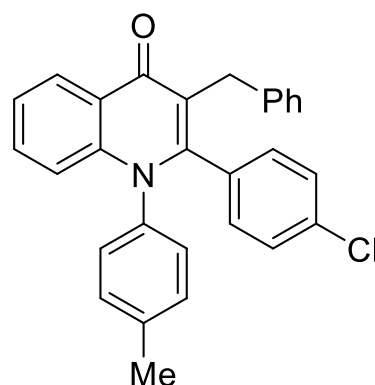

6I

White solid (0.071 g, 81\% yield, m.p. 208.5-210.2 ${ }^{\circ} \mathrm{C}$ ); $\mathrm{R}_{f}=0.5$ (silica, EtOAc:hexanes, 1:4); IR (film) $v_{\max } 3010,1618,1530,1478,1138,980$ $\mathrm{cm}^{-1} ;{ }^{1} \mathrm{H}$ NMR $\left(400 \mathrm{MHz}, \mathrm{CDCl}_{3}\right) \delta=8.56(\mathrm{~d}, J=8.0 \mathrm{~Hz}, 1 \mathrm{H}), 7.44-$ $7.33(\mathrm{~m}, 2 \mathrm{H}), 7.12-7.07(\mathrm{~m}, 7 \mathrm{H}), 6.94(\mathrm{dd}, \mathrm{J}=18.8,7.6 \mathrm{~Hz}, 4 \mathrm{H}), 6.85$ (d, J = 8.0 Hz, 2H), $6.74(\mathrm{~d}, J=8.5 \mathrm{~Hz}, 1 \mathrm{H}), 3.77(\mathrm{~s}, 2 \mathrm{H}), 2.31(\mathrm{~s}, 3 \mathrm{H})$; ${ }^{13} \mathrm{C}$ NMR $\left(101 \mathrm{MHz}, \mathrm{CDCl}_{3}\right) \delta=177.3,150.8,142.1,141.3,138.9$, $136.7,134.2,132.8,131.5,131.0,130.1,129.6,128.4,128.1,127.9,126.6,125.4,125.0,123.4$, 121.5, 118.0, 32.3, $21.1 \mathrm{ppm}$; $\mathrm{HRMS}(\mathrm{m} / \mathrm{z}):[\mathrm{M}+\mathrm{Na}]^{+}$calcd for $\mathrm{C}_{29} \mathrm{H}_{22} \mathrm{CINONa}^{+}$458.1282, Found 458.1268.

\section{3-Benzyl-2-(4-chlorophenyl)-1-(4-fluorophenyl)quinolin-4(1H)-one $(6 \mathrm{~m})$}<smiles>O=c1c(Cc2ccccc2)c(-c2ccc(Cl)cc2)n(-c2ccc(F)cc2)c2ccccc12</smiles>

White solid (0.069 g, 79\% yield, m.p. $161.2-163.5^{\circ} \mathrm{C}$ ); $\mathrm{R}_{f}=0.5$ (silica, EtOAc:hexanes, 1:4); IR (film) $v_{\max } 3010,1617,1540,1476,1240,960$ $\mathrm{cm}^{-1} ;{ }^{1} \mathrm{H}$ NMR $\left(400 \mathrm{MHz}, \mathrm{CDCl}_{3}\right) \delta=8.56(\mathrm{~d}, J=8.0 \mathrm{~Hz}, 1 \mathrm{H}), 7.41(\mathrm{dt}$, $J=32.3,7.6 \mathrm{~Hz}, 2 \mathrm{H}), 7.16-7.04(\mathrm{~m}, 7 \mathrm{H}), 7.02-6.95(\mathrm{~m}, 4 \mathrm{H}), 6.87-$ $6.83(\mathrm{~m}, 2 \mathrm{H}), 6.70(\mathrm{~d}, J=8.5 \mathrm{~Hz}, 1 \mathrm{H}), 3.76(\mathrm{~s}, 2 \mathrm{H}) ;{ }^{13} \mathrm{C}$ NMR $(101$ $\left.\mathrm{MHz}, \mathrm{CDCl}_{3}\right) \delta=177.2,161.9(\mathrm{~d}, J=250.9 \mathrm{~Hz}), 150.6,142.0,141.1$, 
$135.4,134.5,132.5,131.8,131.7,131.7,130.9,128.3,128.2,127.9,126.7,125.5,125.0,123.6$ 121.7, 117.6, $116.6(\mathrm{~d}, J=22.9 \mathrm{~Hz}), 32.2 \mathrm{ppm}$; HRMS $(\mathrm{m} / \mathrm{z}):[\mathrm{M}+\mathrm{Na}]^{+}$calcd for $\mathrm{C}_{28} \mathrm{H}_{19} \mathrm{CIFNONa}{ }^{+}$462.1017, Found 462.1031.

\section{3-Benzyl-1,2-bis(4-chlorophenyl)quinolin-4(1H)-one (6n)}

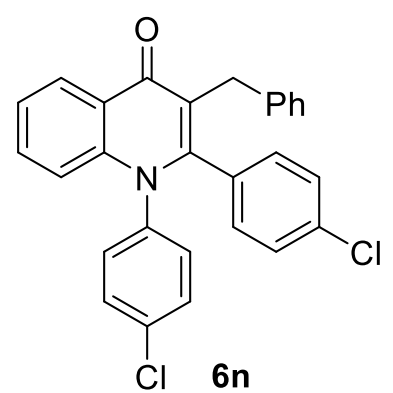

White solid (0.069 g, 76\% yield, m.p. $199.0-201.8^{\circ} \mathrm{C}$ ); $\mathrm{R}_{f}=0.5$ (silica, EtOAc:hexanes, 1:4); IR (film) $v_{\max } 3015,1618,1530,1478,1138,980$ $\mathrm{cm}^{-1} ;{ }^{1} \mathrm{H}$ NMR $\left(400 \mathrm{MHz}, \mathrm{CDCl}_{3}\right) \delta=8.56(\mathrm{~d}, J=8.0 \mathrm{~Hz}, 1 \mathrm{H}), 7.41(\mathrm{dt}$, $J=32.3,7.6 \mathrm{~Hz}, 2 \mathrm{H}), 7.33-7.04(\mathrm{~m}, 7 \mathrm{H}), 6.98(\mathrm{dd}, J=24.0,7.7 \mathrm{~Hz}$, $4 \mathrm{H}), 6.85(\mathrm{~d}, J=8.0 \mathrm{~Hz}, 2 \mathrm{H}), 6.70(\mathrm{~d}, J=8.5 \mathrm{~Hz}, 1 \mathrm{H}), 3.76(\mathrm{~s}, 2 \mathrm{H}) ;{ }^{13} \mathrm{C}$ $\operatorname{NMR}\left(101 \mathrm{MHz}, \mathrm{CDCl}_{3}\right) \delta=177.3,150.3,141.8,141.1,137.9,134.9,134.6,132.4,131.8$ 131.3, 130.9, 129.9, 128.3, 127.9, 126.8, 125.5, 125.0, 123.7, 121.8, 117.5, 32.2 ppm; HRMS (m/z): $[\mathrm{M}+\mathrm{Na}]^{+}$calcd for $\mathrm{C}_{28} \mathrm{H}_{19} \mathrm{Cl}_{2} \mathrm{NONa}^{+}$478.0735, Found 478.0729.

\section{3-Benzyl-2-(4-chlorophenyl)-1-(4-methoxyphenyl)quinolin-4(1H)-one (6o)}

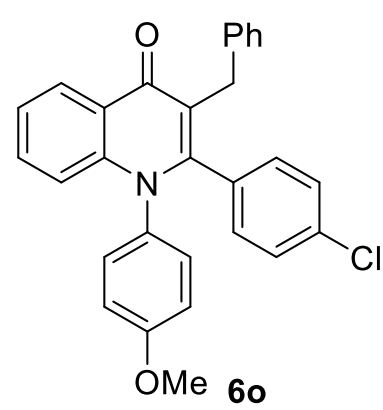

White solid (0.071 g, $78 \%$ yield, m.p. $186.2-187.8^{\circ} \mathrm{C}$ ); $\mathrm{R}_{f}=0.5$ (silica, EtOAc:hexanes, 1:4); IR (film) $v_{\max } 2957,1683,1546,1478,1312,811$ $\mathrm{cm}^{-1} ;{ }^{1} \mathrm{H} \mathrm{NMR}\left(400 \mathrm{MHz}, \mathrm{CDCl}_{3}\right) \delta=8.56(\mathrm{~d}, J=8.0 \mathrm{~Hz}, 1 \mathrm{H}), 7.43(\mathrm{t}, J$ $=7.5 \mathrm{~Hz}, 1 \mathrm{H}), 7.35(\mathrm{t}, J=7.5 \mathrm{~Hz}, 1 \mathrm{H}), 7.13-7.06(\mathrm{~m}, 5 \mathrm{H}), 6.97-6.94$ (m, 4H), $6.85(\mathrm{~d}, J=8.3 \mathrm{~Hz}, 2 \mathrm{H}), 6.77(\mathrm{t}, J=9.1 \mathrm{~Hz}, 3 \mathrm{H}), 3.77(\mathrm{~s}, 5 \mathrm{H})$;

${ }^{13} \mathrm{C}$ NMR $\left(101 \mathrm{MHz}, \mathrm{CDCl}_{3}\right) \delta=177.3,159.3,151.1,142.4,141.3,134.2,132.8,132.0,131.6$, 130.9, 128.4, 128.1, 127.9, 126.6, 125.4, 125.1, 123.5, 121.5, 117.9, 114.6, 55.4, 32.3 ppm; HRMS (m/z): $[\mathrm{M}+\mathrm{Na}]^{+}$calcd for $\mathrm{C}_{28} \mathrm{H}_{22} \mathrm{ClNO}_{2} \mathrm{Na}^{+} 474.1231$, Found 474.1233.

\section{1,3-diBenzyl-2-phenylquinolin-4(1H)-one (6p)}<smiles>O=c1c(Cc2ccccc2)c(-c2ccccc2)n(Cc2ccccc2)c2ccccc12</smiles>

Colorless oil ( $0.044 \mathrm{~g}, 54 \%$ yield); $\mathrm{R}_{f}=0.5$ (silica, EtOAc:hexanes, 1:4); IR (film) $\mathrm{V}_{\max } 2973,1626,1579,1494,1472,766 \mathrm{~cm}^{-1} ;{ }^{1} \mathrm{H}$ NMR $(400 \mathrm{MHz}$, 
$\left.\mathrm{CDCl}_{3}\right) \delta=8.60(\mathrm{dd}, J=8.0,0.9 \mathrm{~Hz}, 1 \mathrm{H}), 7.51(\mathrm{t}, J=11.4 \mathrm{~Hz}, 1 \mathrm{H}), 7.41-7.34(\mathrm{~m}, 2 \mathrm{H}), 7.31-$ $7.21(\mathrm{~m}, 6 \mathrm{H}), 7.12-7.02(\mathrm{~m}, 5 \mathrm{H}), 6.94(\mathrm{~d}, J=7.1 \mathrm{~Hz}, 2 \mathrm{H}), 6.89(\mathrm{~d}, J=6.8 \mathrm{~Hz}, 2 \mathrm{H}), 5.14$ (s, 2H), $3.75(\mathrm{~s}, 2 \mathrm{H}) ;{ }^{13} \mathrm{C}$ NMR $\left(101 \mathrm{MHz}, \mathrm{CDCl}_{3}\right) \delta=177.3,152.8,141.4,140.4,136.3,134.1$, $132.0,129.2,128.8,128.6,128.4,128.3,127.8,127.4,127.2,126.0,125.4,123.4,121.9$, 116.9, 52.2, 32.7 ppm; HRMS (m/z): $[\mathrm{M}+\mathrm{Na}]^{+}$calcd for $\mathrm{C}_{29} \mathrm{H}_{23} \mathrm{NONa}^{+}$424.1671, Found 424.1660.

\section{1,3-diBenzyl-2-(4-chlorophenyl)quinolin-4(1H)-one (6q)}

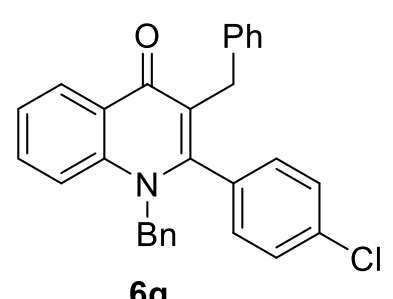

6q

Colorless oil $\left(0.051 \mathrm{~g}, 58 \%\right.$ yield); $\mathrm{R}_{f}=0.5$ (silica, EtOAc:hexanes, 1:4); IR (film) $v_{\max } 2947,1630,1548,1472,1312,760 \mathrm{~cm}^{-1} ;{ }^{1} \mathrm{H}$ NMR $\left(400 \mathrm{MHz}, \mathrm{CDCl}_{3}\right) \delta=8.60(\mathrm{~d}, J=8.0 \mathrm{~Hz}, 1 \mathrm{H}), 7.53(\mathrm{t}, J=7.8 \mathrm{~Hz}, 1 \mathrm{H})$,

$7.38(\mathrm{t}, J=7.5 \mathrm{~Hz}, 1 \mathrm{H}), 7.30(\mathrm{~d}, J=8.7 \mathrm{~Hz}, 2 \mathrm{H}), 7.26-7.23(\mathrm{~m}, 4 \mathrm{H})$, 7.12 (t, $J=7.2 \mathrm{~Hz}, 2 \mathrm{H}), 7.07$ (t, $J=7.2 \mathrm{~Hz}, 1 \mathrm{H}), 6.94(\mathrm{t}, J=6.1 \mathrm{~Hz}, 4 \mathrm{H}), 6.88(\mathrm{~d}, J=7.1 \mathrm{~Hz}$, 2H), $5.12(\mathrm{~s}, 2 \mathrm{H}), 3.74(\mathrm{~s}, 2 \mathrm{H}) ;{ }^{13} \mathrm{C} \operatorname{NMR}\left(101 \mathrm{MHz}, \mathrm{CDCl}_{3}\right) \delta=177.2,151.5,141.2,140.5$, $136.1,135.4,132.5,132.2,129.7,128.9,128.3,127.9,127.6,127.3,126.0,125.5,125.3,123.6$, 122.1, 116.8, 52.2, 32.6 ppm; HRMS (m/z): $\left[\mathrm{M}+\mathrm{Na}^{+}\right.$calcd for $\mathrm{C}_{29} \mathrm{H}_{22} \mathrm{CINONa}^{+}$458.1282, Found 458.1269.

\section{3-Benzyl-1-phenyl-2-(p-tolyl)quinolin-4(1H)-one (6r)}

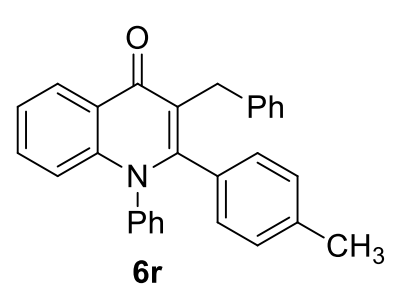

White solid (0.061 g, 75\% yield, m.p. 198.0-200.2 ${ }^{\circ} \mathrm{C}$ ); $\mathrm{R}_{f}=0.5$ (silica, EtOAc:hexanes, 1:4); IR (film) $v_{\max } 2964,1618,1572,1491,1476,760$ $\mathrm{cm}^{-1} ;{ }^{1} \mathrm{H}$ NMR $\left(400 \mathrm{MHz}, \mathrm{CDCl}_{3}\right) \delta=8.56(\mathrm{~d}, J=7.9 \mathrm{~Hz}, 1 \mathrm{H}), 7.41$ (t, $J$ = 7. $4 \mathrm{~Hz}, 1 \mathrm{H}), 7.34(\mathrm{t}, J=7.4 \mathrm{~Hz}, 1 \mathrm{H}), 7.26-7.28(\mathrm{~m}, 3 \mathrm{H}), 7.14-$ $7.02(\mathrm{~m}, 7 \mathrm{H}), 6.93(\mathrm{~d}, J=7.6 \mathrm{~Hz}, 2 \mathrm{H}), 6.82(\mathrm{~d}, J=7.6 \mathrm{~Hz}, 2 \mathrm{H}), 6.72(\mathrm{~d}, J=8.5 \mathrm{~Hz}, 1 \mathrm{H}), 3.79$ (s, 2H), $2.24(\mathrm{~s}, 3 \mathrm{H}) ;{ }^{13} \mathrm{C} \mathrm{NMR}\left(101 \mathrm{MHz}, \mathrm{CDCl}_{3}\right) \delta=177.4,152.1,141.9,141.6,139.7,137.9$, 
$131.4,131.3,130.1,129.5,129.3,128.5,128.4,127.8,126.6,125.3,125.0,123.3,121.5,117.9$, 32.6, $21.2 \mathrm{ppm}$; HRMS (m/z): [M + Na] ${ }^{+}$calcd for $\mathrm{C}_{29} \mathrm{H}_{23} \mathrm{NONa}^{+}$424.1671, Found 424.1666.

\section{3-Benzyl-2-(4-fluorophenyl)-1-phenylquinolin-4(1H)-one (6s)}

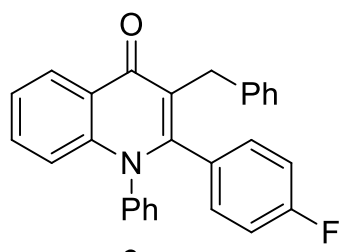

6s

White solid (0.062 g, 76\% yield, m.p. 198.3-200.0 ${ }^{\circ} \mathrm{C}$ ); $\mathrm{R}_{f}=0.5$ (silica, EtOAc:hexanes, 1:4); IR (film) $v_{\max } 3054,2838,1590,1526,1493,1382$, $1169 \mathrm{~cm}^{-1} ;{ }^{1} \mathrm{H}$ NMR $\left(400 \mathrm{MHz}, \mathrm{CDCl}_{3}\right) \delta=8.58(\mathrm{~d}, J=7.9 \mathrm{~Hz}, 1 \mathrm{H}), 7.44$ (t, J=7.6 Hz, 1H), $7.36(t, J=7.4 \mathrm{~Hz}, 1 \mathrm{H}), 7.30-7.26(\mathrm{~m}, 3 \mathrm{H}), 7.14-$ $7.05(\mathrm{~m}, 5 \mathrm{H}), 6.97(\mathrm{~d}, J=7.2 \mathrm{~Hz}, 2 \mathrm{H}), 6.90-6.87(\mathrm{~m}, 2 \mathrm{H}), 6.82(\mathrm{t}, J=8.4 \mathrm{~Hz}, 2 \mathrm{H}), 6.74(\mathrm{~d}, J=$ $8.5 \mathrm{~Hz}, 1 \mathrm{H}), 3.80(\mathrm{~s}, 2 \mathrm{H}) ;{ }^{13} \mathrm{C} \operatorname{NMR}\left(101 \mathrm{MHz}, \mathrm{CDCl}_{3}\right) \delta=177.4,162.08(\mathrm{~d}, J=249.2 \mathrm{~Hz})$, 150.9, 141.9, 139.5, 131.6, 130.3 (d, $J=3.7 \mathrm{~Hz}), 129.5,128.8,128.4,127.9,126.6,125.5$, 125.03, 123.5, 123.3 (d, $J=153.2 \mathrm{~Hz}), 117.9,114.9$ (d, $J=21.8 \mathrm{~Hz}), 32.36 \mathrm{ppm}$; HRMS (m/z): $[\mathrm{M}+\mathrm{Na}]^{+}$calcd for $\mathrm{C}_{28} \mathrm{H}_{20} \mathrm{FNONa}^{+}$428.1421, Found 428.1410.

\section{3-Benzyl-1-phenyl-2-(4-(trifluoromethyl)phenyl)quinolin-4(1H)-one (6t)}

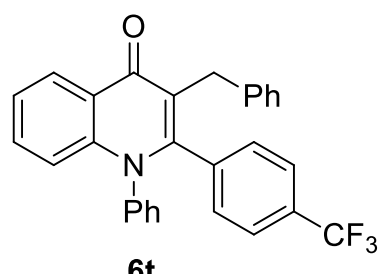

6t

White solid (0.072 g, 79\% yield, m.p. $\left.185.8-187.2^{\circ} \mathrm{C}\right) ; \mathrm{R}_{f}=0.5$ (silica, EtOAc:hexanes, 1:4); IR (film) $\mathrm{v}_{\max } 3057,2973,1598,1547,1470$, 1328, 1169, $760 \mathrm{~cm}^{-1} ;{ }^{1} \mathrm{H}$ NMR $\left(400 \mathrm{MHz} \mathrm{CDCl}_{3}\right) \delta=8.58(\mathrm{~d}, J=7.8$ $\mathrm{Hz}, 1 \mathrm{H}), 7.46(\mathrm{t}, J=7.3 \mathrm{~Hz}, 1 \mathrm{H}), 7.40-7.37(\mathrm{~m}, 3 \mathrm{H}), 7.28(\mathrm{t}, J=7.2$ $\mathrm{Hz}, 3 \mathrm{H}), 7.12-7.03(\mathrm{~m}, 7 \mathrm{H}), 6.92(\mathrm{~d}, J=6.9 \mathrm{~Hz}, 2 \mathrm{H}), 6.75(\mathrm{~d}, J=8.5 \mathrm{~Hz}, 1 \mathrm{H}), 3.77(\mathrm{~s}, 2 \mathrm{H}) ;{ }^{13} \mathrm{C}$ $\operatorname{NMR}\left(101 \mathrm{MHz}, \mathrm{CDCl}_{3}\right) \delta=177.3,150.3,141.9,141.1,139.2,137.8,131.8,130.1,129.7$ 129.0, 128.3, 127.9, 126.7, 125.6, 125.0, 124.8 (q, J = 3.7 Hz), 123.7, 121.5, 117.9, 32.22 ppm; HRMS (m/z): $[\mathrm{M}+\mathrm{Na}]^{+}$calcd for $\mathrm{C}_{29} \mathrm{H}_{20} \mathrm{~F}_{3} \mathrm{NONa}^{+}$478.1389, Found 478.1389.

3-Benzyl-1-phenyl-2-(4-(trifluoromethoxy)phenyl)quinolin-4(1H)-one (6u) 


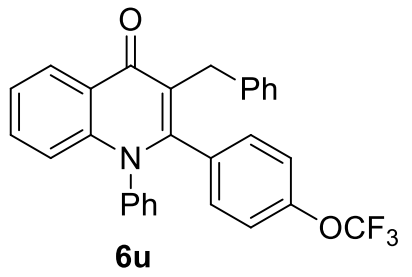

White solid $\left(0.071 \mathrm{~g}, 75 \%\right.$ yield, m.p. $\left.178.2-179.5{ }^{\circ} \mathrm{C}\right) ; \mathrm{R}_{f}=0.5$ (silica, EtOAc:hexanes, 1:4); IR (film) $v_{\max } 3056,2964,1641,1603$, $1460,1329,1070,709 \mathrm{~cm}^{-1} ;{ }^{1} \mathrm{H}$ NMR $\left(400 \mathrm{MHz}, \mathrm{CDCl}_{3}\right) \delta=8.58(\mathrm{~d}, J$ $=7.8 \mathrm{~Hz}, 1 \mathrm{H}), 7.44(\mathrm{t}, J=7.5 \mathrm{~Hz}, 1 \mathrm{H}), 7.37(\mathrm{t}, J=7.4 \mathrm{~Hz}, 1 \mathrm{H}), 7.27$ (t, $J=6.6 \mathrm{~Hz}, 3 \mathrm{H}), 7.10-7.04(\mathrm{~m}, 5 \mathrm{H}), 6.97-6.91(\mathrm{~m}, 6 \mathrm{H}), 6.75(\mathrm{~d}, J=8.5 \mathrm{~Hz}, 1 \mathrm{H}), 3.81(\mathrm{~s}$, $2 \mathrm{H}) ;{ }^{13} \mathrm{C}$ NMR $\left(101 \mathrm{MHz}, \mathrm{CDCl}_{3}\right) \delta=177.3,150.6,148.7,141.9,141.2,139.3,132.8,131.7$, 131.3, 130.0, 129.54, 128.8, 128.4, 127.9, 126.7, 125.5, 125.0, 123.6, 121.7, 121.5, 120.2, 117.9, 32.2 ppm; HRMS (m/z): [M + Na] $]^{+}$calcd for $\mathrm{C}_{29} \mathrm{H}_{20} \mathrm{~F}_{3} \mathrm{NO}_{2} \mathrm{Na}^{+}$494.1338, Found 494.1327.

\section{3-Benzyl-2-(4-nitrophenyl)-1-phenylquinolin-4(1H)-one (6v)}

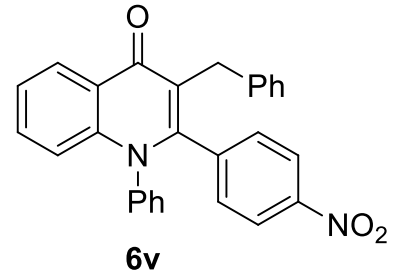

6v

Brown solid $\left(0.070 \mathrm{~g}, 81 \%\right.$ yield, m.p. $\left.251.2-252.6^{\circ} \mathrm{C}\right) ; \mathrm{R}_{f}=0.5$ (silica, EtOAc:hexanes, 1:4); IR (film) $v_{\max }$ 2949, 1619, 1550, 1524, 1478, 1356, $756 \mathrm{~cm}^{-1} ;{ }^{1} \mathrm{H}$ NMR $\left(400 \mathrm{MHz}, \mathrm{CDCl}_{3}\right) \delta=8.57(\mathrm{~d}, J=8.0 \mathrm{~Hz}$, $1 \mathrm{H}), 7.97(\mathrm{~d}, J=8.5 \mathrm{~Hz}, 2 \mathrm{H}), 7.49-7.43(\mathrm{~m}, 1 \mathrm{H}), 7.41-7.35(\mathrm{~m}, 1 \mathrm{H})$, $7.31-7.26(\mathrm{~m}, 3 \mathrm{H}), 7.13-7.05(\mathrm{~m}, 7 \mathrm{H}), 6.90(\mathrm{~d}, J=7.1 \mathrm{~Hz}, 2 \mathrm{H}), 6.75(\mathrm{~d}, J=8.5 \mathrm{~Hz}, 1 \mathrm{H}), 3.75$ $(\mathrm{s}, 2 \mathrm{H}) ;{ }^{13} \mathrm{C}$ NMR $\left(101 \mathrm{MHz}, \mathrm{CDCl}_{3}\right) \delta=177.1,149.4,147.1,141.9,140.8,140.5,138.9,131.9$, 130.8, 129.9, 129.8, 129.3, 128.1, 128.0, 126.6, 125.7, 124.9, 123.8, 122.9, 121.2, 117.8, 32.02 ppm; HRMS (m/z): [M + Na] $]^{+}$calcd for $\mathrm{C}_{28} \mathrm{H}_{20} \mathrm{~N}_{2} \mathrm{O}_{3} \mathrm{Na}^{+}$455.1366, Found 455.1367.

\section{4-(3-Benzyl-4-oxo-1-phenyl-1,4-dihydroquinolin-2-yl)benzonitrile (6w)}

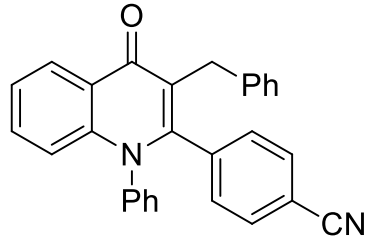

6w

Yellow solid (0.068 g, 82\% yield, m.p. $\left.215.2-217.5^{\circ} \mathrm{C}\right) ; \mathrm{R}_{f}=0.5$ (silica, EtOAc:hexanes, 1:4); IR (film) $v_{\max }$ 3059, 2248, 1628, 1549, 1496, 1315, $754 \mathrm{~cm}^{-1} ;{ }^{1} \mathrm{H} \mathrm{NMR}\left(400 \mathrm{MHz}, \mathrm{CDCl}_{3}\right) \delta=8.57(\mathrm{~d}, J=7.8 \mathrm{~Hz}, 1 \mathrm{H})$, $7.47-7.36(\mathrm{~m}, 4 \mathrm{H}), 7.28(\mathrm{~d}, J=12.9 \mathrm{~Hz}, 3 \mathrm{H}), 7.10-7.02(\mathrm{~m}, 7 \mathrm{H})$, $6.89(\mathrm{~d}, J=6.6 \mathrm{~Hz}, 2 \mathrm{H}), 6.75(\mathrm{~d}, J=8.4 \mathrm{~Hz}, 1 \mathrm{H}), 3.75(\mathrm{~s}, 2 \mathrm{H}) ;{ }^{13} \mathrm{C} \mathrm{NMR}\left(101 \mathrm{MHz}, \mathrm{CDCl}_{3}\right) \delta=$ $177.2,149.8,141.9,140.9,138.9,138.6,131.9,131.5,130.5,129.9,129.7,129.2,128.1,128.0$ 
126.6, 125.6, 124.9, 123.8, 121.2, 117.9, 112.2, 32.0 ppm; HRMS (m/z): $[\mathrm{M}+\mathrm{Na}]^{+}$calcd for $\mathrm{C}_{29} \mathrm{H}_{20} \mathrm{~N}_{2} \mathrm{ONa}^{+}$435.1467, Found 435.1463.

\section{3-Benzyl-2-(3,5-difluorophenyl)-1-phenylquinolin-4(1H)-one (6x)}

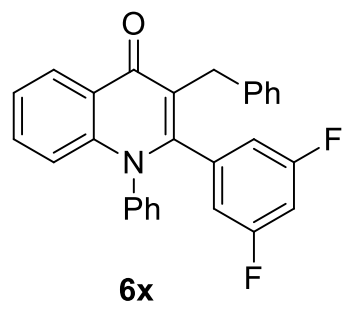

White solid (0.060 g, 78\% yield, m.p. 297.2-299.1 ${ }^{\circ} \mathrm{C}$ ); $\mathrm{R}_{f}=0.5$ (silica, EtOAc:hexanes, 1:4); IR (film) $v_{\max } 3052,2964,1618,1497,1338,1098$, $1026 \mathrm{~cm}^{-1} ;{ }^{1} \mathrm{H}$ NMR $\left(400 \mathrm{MHz}, \mathrm{CDCl}_{3}\right) \delta=8.57(\mathrm{~d}, \mathrm{~J}=8.0 \mathrm{~Hz}, 1 \mathrm{H}), 7.45$ (t, $J=7.7 \mathrm{~Hz}, 1 \mathrm{H}), 7.40-7.31(\mathrm{~m}, 4 \mathrm{H}), 7.16-7.08(\mathrm{~m}, 5 \mathrm{H}), 6.98(\mathrm{~d}, J=$ $7.5 \mathrm{~Hz}, 2 \mathrm{H}), 6.73(\mathrm{~d}, J=8.5 \mathrm{~Hz}, 1 \mathrm{H}), 6.62(\mathrm{t}, J=8.9 \mathrm{~Hz}, 1 \mathrm{H}), 6.45(\mathrm{~d}, J=5.6 \mathrm{~Hz}, 2 \mathrm{H}), 3.81$ (s, $2 \mathrm{H}) ;{ }^{13} \mathrm{C}$ NMR $\left(101 \mathrm{MHz}, \mathrm{CDCl}_{3}\right) \delta=177.3,162.2(\mathrm{dd}, J=251.0,12.8 \mathrm{~Hz}), 149.1,141.9,141.0$, 139.0, 136.9, 131.9, 129.9, 129.7, 129.1, 128.3, 128.0, 126.7, 125.7, 125.1, 123.7, 121.4, 117.9, $113.3(\mathrm{~d}, J=7.8 \mathrm{~Hz}), 113.1(\mathrm{~d}, J=7.3 \mathrm{~Hz}), 104.2,103.9$ (t, $J=25.0 \mathrm{~Hz}) \mathrm{ppm}$; HRMS (m/z): [M + $\mathrm{Na}]^{+}$calcd for $\mathrm{C}_{28} \mathrm{H}_{19} \mathrm{~F}_{2} \mathrm{NONa}^{+}$446.1326, Found 446.1316.

\section{3-Benzyl-2-(2,5-dimethoxyphenyl)-1-phenylquinolin-4(1H)-one (6y)}

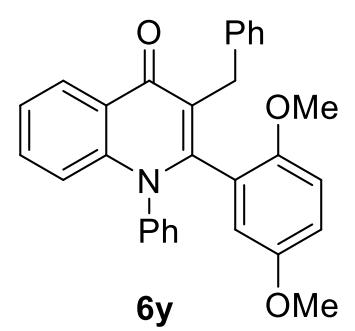

White solid (0.070 g, $78 \%$ yield, m.p. 182.1-183.4 ${ }^{\circ} \mathrm{C}$ ); $\mathrm{R}_{f}=0.5$ (silica,

EtOAc:hexanes, 1:4); IR (film) $v_{\max }$ 2958, 1583, 1576, 1498,1317, 819 $\mathrm{cm}^{-1} ;{ }^{1} \mathrm{H}$ NMR $\left(400 \mathrm{MHz}, \mathrm{CDCl}_{3}\right) \delta=8.58(\mathrm{~d}, J=7.8 \mathrm{~Hz}, 1 \mathrm{H}), 7.37(\mathrm{dt}, J=$ 14.7, 7.1 Hz, 2H), $7.26(\mathrm{~d}, J=2.9 \mathrm{~Hz}, 4 \mathrm{H}), 7.11-6.98(\mathrm{~m}, 6 \mathrm{H}), 6.73-$ $6.67(\mathrm{~m}, 2 \mathrm{H}), 6.50(\mathrm{~d}, J=9.0 \mathrm{~Hz}, 1 \mathrm{H}), 6.37(\mathrm{~d}, J=2.5 \mathrm{~Hz}, 1 \mathrm{H}), 3.90(\mathrm{~d}, J$ $=14.5 \mathrm{~Hz}, 1 \mathrm{H}), 3.68(\mathrm{~d}, J=14.5 \mathrm{~Hz}, 1 \mathrm{H}), 3.52(\mathrm{~s}, 3 \mathrm{H}), 3.42(\mathrm{~s}, 3 \mathrm{H}) ;{ }^{13} \mathrm{C} \mathrm{NMR}\left(101 \mathrm{MHz}, \mathrm{CDCl}_{3}\right)$ $\delta=177.5,152.6,149.6,149.3,142.0,141.8,139.4,131.2,129.8,128.7,128.6,127.6,126.6$, 125.2, 123.7, 123.2, 121.6, 117.8, 116.1, 115.8, 111.2, 55.6, 54.9, 32.5 ppm; HRMS (m/z): [M + $\mathrm{Na}]^{+}$calcd for $\mathrm{C}_{30} \mathrm{H}_{25} \mathrm{NO}_{3} \mathrm{Na}^{+} 470.1726$, Found 470.1712 .

\section{3-Benzyl-2-(3-chlorophenyl)-1-phenylquinolin-4(1H)-one (6z)}


<smiles>O=c1c(Cc2ccccc2)c(-c2cccc(Cl)c2)n(-c2ccccc2)c2ccccc12</smiles>

$6 z$

White solid (0.066 g, 78\% yield, m.p. $264.0-265.0^{\circ} \mathrm{C}$ ); $\mathrm{R}_{f}=0.5$ (silica, EtOAc:hexanes, 1:4); IR (film) $v_{\max } 2949,1620,1542,1475,1302,756$ $\mathrm{cm}^{-1} ;{ }^{1} \mathrm{H}$ NMR $\left(400 \mathrm{MHz}, \mathrm{CDCl}_{3}\right) \delta=8.58(\mathrm{~d}, J=7.6 \mathrm{~Hz}, 1 \mathrm{H}), 7.44(\mathrm{t}, J$ $=7.2 \mathrm{~Hz}, 1 \mathrm{H}), 7.37-7.26(\mathrm{~m}, 4 \mathrm{H}), 7.15-7.05(\mathrm{~m}, 7 \mathrm{H}), 6.97(\mathrm{~d}, J=$

$7.2 \mathrm{~Hz}, 2 \mathrm{H}), 6.87(\mathrm{~s}, 1 \mathrm{H}), 6.82(\mathrm{~d}, J=7.5 \mathrm{~Hz}, 1 \mathrm{H}), 6.74(\mathrm{~d}, J=8.5 \mathrm{~Hz}, 1 \mathrm{H}), 3.86(\mathrm{~d}, J=14.5 \mathrm{~Hz}$ $1 \mathrm{H}), 3.73(\mathrm{~d}, J=14.5 \mathrm{~Hz}, 1 \mathrm{H}) ;{ }^{13} \mathrm{C} \operatorname{NMR}\left(101 \mathrm{MHz}, \mathrm{CDCl}_{3}\right) \delta=177.3,150.3,141.9,141.2$, 139.2, 135.7, 133.7, 131.7, 130.2, 129.9, 129.8, 129.5, 129.4, 129.1, 128.9, 128.4, 127.9, 127.8, 126.6, 125.5, 125.0, 123.5, 121.5, 117.9, 32.3 ppm; HRMS (m/z): $[\mathrm{M}+\mathrm{Na}]^{+}$calcd for $\mathrm{C}_{28} \mathrm{H}_{20} \mathrm{CINONa}^{+}$444.1125, Found 444.1113.

\section{3-Benzyl-2-(4-fluorophenyl)-1-phenylquinolin-4(1H)-one (6aa)}<smiles>O=c1c(Cc2ccccc2)c(-c2ccccc2F)n(-c2ccccc2)c2ccccc12</smiles>

6aa

White solid (0.062 g, 76\% yield, m.p. 189.7-191.6 $\left.{ }^{\circ} \mathrm{C}\right) ; \mathrm{R}_{f}=0.5$ $\mathrm{Na}$

(silica, EtOAc:hexanes, 1:4); IR (film) $v_{\max } 3056,2848,1596,1528$, 1496, 1379, $1173 \mathrm{~cm}^{-1} ;{ }^{1} \mathrm{H} \operatorname{NMR}\left(400 \mathrm{MHz} \mathrm{CDCl}_{3}\right) \delta=8.57$ (dd, $J=$ 8.0, 1.7 Hz, 1H), $7.48-7.33(\mathrm{~m}, 2 \mathrm{H}), 7.32-7.25(\mathrm{~m}, 4 \mathrm{H}), 7.18(\mathrm{td}, J$ $=8.7,8.2,4.6 \mathrm{~Hz}, 1 \mathrm{H}), 7.14-7.00(\mathrm{~m}, 4 \mathrm{H}), 7.01-6.86(\mathrm{~m}, 4 \mathrm{H}), 6.82(\mathrm{t}, J=8.9 \mathrm{~Hz}, 1 \mathrm{H}), 6.73$ $(\mathrm{d}, J=8.5 \mathrm{~Hz}, 1 \mathrm{H}), 3.93(\mathrm{~d}, J=14.6 \mathrm{~Hz}, 1 \mathrm{H}), 3.69(\mathrm{~d}, J=14.6 \mathrm{~Hz}, 1 \mathrm{H}) ;{ }^{13} \mathrm{C} \mathrm{NMR}(101 \mathrm{MHz}$, $\left.\mathrm{CDCl}_{3}\right) \delta=177.3,158.4(\mathrm{~d}, J=247.4 \mathrm{~Hz}), 146.4,142.1,140.9,139.4,131.6,131.08(\mathrm{~d}, J=8.3$ Hz), 129.7 (d, $J=20.0 \mathrm{~Hz}), 129.8,129.6,129.3,129.0,128.9,128.4,127.8,126.7,125.4$, 125.1, 123.6 (d, $J=20.7 \mathrm{~Hz}), 122.4,117.9,115.6,115.4,109.9,32.5$ ppm; HRMS (m/z): [M + $\mathrm{Na}]^{+}$calcd for $\mathrm{C}_{28} \mathrm{H}_{20} \mathrm{FNONa}^{+}$428.1421, Found 428.1417.

\section{3-Benzyl-2-(2-chlorophenyl)-1-phenylquinolin-4(1H)-one (6ab)}<smiles>O=c1c(Cc2ccccc2)c(-c2ccccc2Cl)n(-c2ccccc2)c2ccccc12</smiles>

$6 \mathrm{ab}$

White solid (0.064 g, 75\% yield, m.p. 162.3-164.6 ${ }^{\circ} \mathrm{C}$ ); $\mathrm{R}_{f}=0.5$ (silica, EtOAc:hexanes, 1:4); IR (film) $v_{\max } 2953,1628,1576,1491,1311,766 \mathrm{~cm}^{-}$ 1; ${ }^{1} \mathrm{H}$ NMR $\left(400 \mathrm{MHz}, \mathrm{CDCl}_{3}\right) \delta=8.59(\mathrm{~d}, J=7.7 \mathrm{~Hz}, 1 \mathrm{H}), 7.45-7.35(\mathrm{~m}$, 
3H), $7.30-7.21(\mathrm{~m}, 4 \mathrm{H}), 7.14-7.06(\mathrm{~m}, 5 \mathrm{H}), 7.00-6.93(\mathrm{~m}, 3 \mathrm{H}), 6.82(\mathrm{~d}, J=7.4 \mathrm{~Hz}, 1 \mathrm{H})$, $6.72(\mathrm{~d}, J=8.4 \mathrm{~Hz}, 1 \mathrm{H}), 4.21(\mathrm{~d}, J=14.6 \mathrm{~Hz}, 1 \mathrm{H}), 3.33(\mathrm{~d}, J=14.6 \mathrm{~Hz}, 1 \mathrm{H}) ;{ }^{13} \mathrm{C}$ NMR $(101$ $\left.\mathrm{MHz}_{\mathrm{CDCl}}\right) \delta=177.5,148.8,142.1,140.9,139.1,133.2,132.9,132.0,131.6,130.1,129.9$ $129.5,129.3,129.1,128.5,128.4,127.8,126.6,126.0,125.4,125.2,123.5,121.9,117.9,32.2$ ppm; HRMS (m/z): [M + Na] $]^{+}$calcd for $\mathrm{C}_{28} \mathrm{H}_{20} \mathrm{CINONa}^{+}$444.1125, Found 444.1114.

\section{3-Benzyl-2-(4-bromo-3-fluorophenyl)-1-phenylquinolin-4(1H)-one (6ac)}

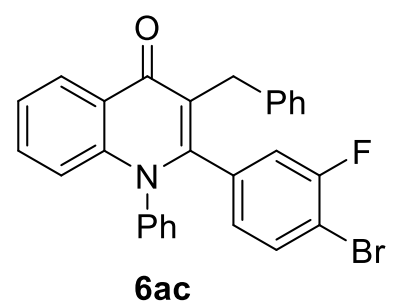

White solid (0.072 g, 75\% yield, m.p. 206.2-208.6 ${ }^{\circ} \mathrm{C}$ ); $\mathrm{R}_{f}=0.5$ (silica, EtOAc:hexanes, 1:4); IR (film) $v_{\max } 2908,1636,1573,1481,1437$, 1098, $1026 \mathrm{~cm}^{-1} ;{ }^{1} \mathrm{H} \mathrm{NMR}\left(400 \mathrm{MHz}, \mathrm{CDCl}_{3}\right) \delta=8.56(\mathrm{~d}, J=7.7 \mathrm{~Hz}$, $1 \mathrm{H}), 7.47-7.43(\mathrm{~m}, 1 \mathrm{H}), 7.39-7.26(\mathrm{~m}, 5 \mathrm{H}), 7.14-7.08(\mathrm{~m}, 5 \mathrm{H})$, $6.96(\mathrm{~d}, J=6.9 \mathrm{~Hz}, 2 \mathrm{H}), 6.73(\mathrm{~d}, J=8.4 \mathrm{~Hz}, 1 \mathrm{H}), 6.63(\mathrm{dd}, J=19.6,8.3 \mathrm{~Hz}, 2 \mathrm{H}), 3.79(\mathrm{q}, J=$ 14.7 Hz, 2H); ${ }^{13} \mathrm{C}$ NMR (101 MHz, $\left.\mathrm{CDCl}_{3}\right) \delta=177.3,158.2(\mathrm{~d}, J=249.7 \mathrm{~Hz}), 149.3,141.9$, 141.0, 139.1, 135.2 (d, $J=6.9 \mathrm{~Hz}), 133.1,131.8,129.9,129.7$ (d, $J=9.6 \mathrm{~Hz}), 129.1,128.3$, 128.0, 126.7, 125.6, 124.9, 123.7, 121.5, 118.1, 117.9, 109.3 (d, $J=21.0 \mathrm{~Hz}), 32.2$ ppm; HRMS (m/z): $[\mathrm{M}+\mathrm{Na}]^{+}$calcd for $\mathrm{C}_{28} \mathrm{H}_{19} \mathrm{BrFNONa}^{+}$506.0526, Found 506.0516.

\section{3-Benzyl-2-(perfluorophenyl)-1-phenylquinolin-4(1H)-one (6ad)}

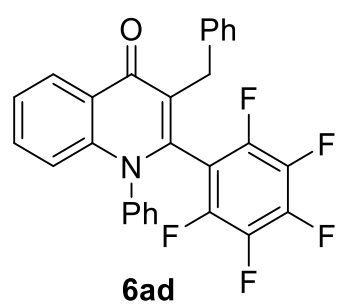

White solid (0.095 g, $82 \%$ yield, m.p. $212.3-215.8{ }^{\circ} \mathrm{C}$ ); $\mathrm{R}_{f}=0.5$ (silica, EtOAc:hexanes, 1:4); IR (film) $v_{\max } 2968,1643,1593,1470,1323,1169$, $1014 \mathrm{~cm}^{-1} ;{ }^{1} \mathrm{H}$ NMR $\left(400 \mathrm{MHz}, \mathrm{CDCl}_{3}\right) \delta=8.58(\mathrm{~d}, J=7.7 \mathrm{~Hz}, 1 \mathrm{H}), 7.48$ $(\mathrm{t}, J=7.3 \mathrm{~Hz}, 1 \mathrm{H}), 7.42-7.40(\mathrm{~m}, 4 \mathrm{H}), 7.22(\mathrm{~s}, 2 \mathrm{H}), 7.11(\mathrm{~d}, J=7.1 \mathrm{~Hz}$,

$3 \mathrm{H}), 6.91(\mathrm{~d}, J=6.4 \mathrm{~Hz}, 2 \mathrm{H}), 6.72(\mathrm{~d}, J=8.5 \mathrm{~Hz}, 1 \mathrm{H}), 3.86(\mathrm{~s}, 2 \mathrm{H}) ;{ }^{13} \mathrm{C} \mathrm{NMR}\left(101 \mathrm{MHz}, \mathrm{CDCl}_{3}\right)$ $\delta=176.9,142.83(\mathrm{~d}, J=246.5 \mathrm{~Hz}), 142.2,139.7,138.9,137.2(\mathrm{~d}, J=257.9 \mathrm{~Hz}), 132.2,130.1$, $128.1(\mathrm{~d}, J=4.7 \mathrm{~Hz}), 127.8,126.8,125.9,124.9,124.1,117.8,32.1$ ppm; HRMS (m/z): [M + $\mathrm{Na}]^{+}$calcd for $\mathrm{C}_{28} \mathrm{H}_{16} \mathrm{~F}_{5} \mathrm{NONa}^{+}$500.1044, Found 500.1029. 


\section{3-Benzyl-1-phenyl-2-(thiophen-2-yl)quinolin-4(1H)-one (6ae)}

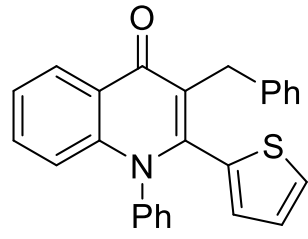

6ae

White solid (0.054 g, 69\% yield, m.p. $\left.144.4-146.7{ }^{\circ} \mathrm{C}\right) ; \mathrm{R}_{f}=0.5$ (silica, EtOAc:hexanes, 1:4); IR (film) $v_{\max }$ 3039, 1629, 1528, 1479, 1402, 1386, $749 \mathrm{~cm}^{-1} ;{ }^{1} \mathrm{H} \mathrm{NMR}\left(400 \mathrm{MHz}, \mathrm{CDCl}_{3}\right) \delta=8.54(\mathrm{~d}, J=7.8 \mathrm{~Hz}, 1 \mathrm{H}), 7.43(\mathrm{t}, J$ $=7.2 \mathrm{~Hz}, 1 \mathrm{H}), 7.37-7.33(\mathrm{~m}, 4 \mathrm{H}), 7.26(\mathrm{~s}, 1 \mathrm{H}), 7.22(\mathrm{~d}, J=4.9 \mathrm{~Hz}, 1 \mathrm{H})$,

$7.16-7.09(\mathrm{~m}, 6 \mathrm{H}), 6.78-6.73(\mathrm{~m}, 2 \mathrm{H}), 6.63(\mathrm{~d}, J=2.9 \mathrm{~Hz}, 1 \mathrm{H}), 3.91(\mathrm{~s}, 2 \mathrm{H}) ;{ }^{13} \mathrm{C}$ NMR $(101$ $\left.\mathrm{MHz}, \mathrm{CDCl}_{3}\right) \delta=177.2,142.3,141.4,139.7,134.1,131.7,130.5,130.1,129.3,128.8,128.5$ 127.9, 126.7, 126.3, 125.5, 125.0, 124.1, 123.5, 118.0, 32.9 ppm; HRMS (m/z): [M + Na] $]^{+}$calcd for $\mathrm{C}_{26} \mathrm{H}_{19} \mathrm{NOSNa}^{+}$416.1079, Found 416.1067.

\section{3-Benzyl-2-(naphthalen-1-yl)-1-phenylquinolin-4(1H)-one (6af)}<smiles>O=c1c(Cc2ccccc2)c(-c2cccc3ccccc23)n(-c2ccccc2)c2ccccc12</smiles>

6af White solid (0.067 g, 76\% yield, m.p. 212.0-214.0 ${ }^{\circ} \mathrm{C}$ ); $\mathrm{R}_{f}=0.5$ (silica, EtOAc:hexanes, 1:4); IR (film) $v_{\max }$ 2938, 1637, 1598, 1506, 1473, 1410, $794 \mathrm{~cm}^{-1} ;{ }^{1} \mathrm{H}$ NMR $\left(400 \mathrm{MHz}, \mathrm{CDCl}_{3}\right) \delta=8.65(\mathrm{~d}, J=7.9 \mathrm{~Hz}, 1 \mathrm{H})$, $7.73(\mathrm{~d}, J=8.1 \mathrm{~Hz}, 1 \mathrm{H}), 7.67(\mathrm{~d}, J=8.2 \mathrm{~Hz}, 1 \mathrm{H}), 7.54(\mathrm{~d}, J=8.3 \mathrm{~Hz}$, 1H), $7.46-7.34(\mathrm{~m}, 4 \mathrm{H}), 7.21-7.17(\mathrm{~m}, 2 \mathrm{H}), 7.12(\mathrm{~d}, J=7.9 \mathrm{~Hz}, 1 \mathrm{H}), 7.06(\mathrm{t}, J=7.0 \mathrm{~Hz}, 1 \mathrm{H})$, $6.99-6.96(\mathrm{~m}, 4 \mathrm{H}), 6.87-6.80(\mathrm{~m}, 4 \mathrm{H}), 6.72(\mathrm{~d}, J=8.2 \mathrm{~Hz}, 1 \mathrm{H}), 4.08(\mathrm{~d}, J=14.4 \mathrm{~Hz}, 1 \mathrm{H})$, $3.24(\mathrm{~d}, J=14.4 \mathrm{~Hz}, 1 \mathrm{H}) ;{ }^{13} \mathrm{C} \operatorname{NMR}\left(101 \mathrm{MHz} \mathrm{CDCl}_{3}\right) \delta=177.4,150.1,142.2,141.3,139.2$, 132.8, 131.5, 131.3, 130.8, 130.1, 129.0, 128.9, `18.8, 128.7, 128.5, 128.2, 127.6, 126.6, 126.1, 125.3, 125.0, 124.4, 123.5, 122.6, 117.9, $32.4 \mathrm{ppm}$; HRMS (m/z): $\left[\mathrm{M}+\mathrm{Na}^{+}\right.$calcd for $\mathrm{C}_{32} \mathrm{H}_{23} \mathrm{NONa}^{+} 460.1671$, Found 460.1660 .

\section{3-Benzyl-1-phenylquinolin-4(1H)-one (6ag)}

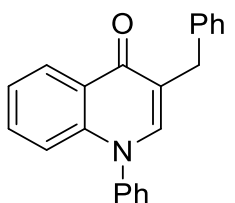

$6 a g$

White solid (0.046 g, 74\% yield, m.p. 177.7-179.1 ${ }^{\circ} \mathrm{C}$ ); $\mathrm{R}_{f}=0.5$ (silica, EtOAc:hexanes, 1:4); IR (film) $v_{\max } 3051,1626,1534,1496,1478,1314,759$ $\mathrm{cm}^{-1} ;{ }^{1} \mathrm{H}$ NMR $\left(400 \mathrm{MHz}, \mathrm{CDCl}_{3}\right) \delta=8.51(\mathrm{~d}, J=7.9 \mathrm{~Hz}, 1 \mathrm{H}), 7.55-7.52(\mathrm{~m}$, 
3H), $7.47-7.43(\mathrm{~m}, 1 \mathrm{H}), 7.39(\mathrm{~s}, 1 \mathrm{H}), 7.35-7.32(\mathrm{~m}, 5 \mathrm{H}), 7.27(\mathrm{t}, J=7.0 \mathrm{~Hz}, 2 \mathrm{H}), 7.18(\mathrm{~d}, J=$ $7.1 \mathrm{~Hz}, 1 \mathrm{H}), 6.97(\mathrm{~d}, J=8.5 \mathrm{~Hz}, 1 \mathrm{H}), 3.96(\mathrm{~s}, 2 \mathrm{H}) ;{ }^{13} \mathrm{C} \mathrm{NMR}\left(101 \mathrm{MHz}, \mathrm{CDCl}_{3}\right) \delta=177.2,141.4$, $140.9,140.2,131.4,130.1,129.2,129.0,128.4,127.6,126.7,126.0,125.4,123.5,122.3,116.9$, $33.54 \mathrm{ppm}$; HRMS (m/z): [M + Na] $]^{+}$calcd for $\mathrm{C}_{22} \mathrm{H}_{17} \mathrm{NONa}^{+} 334.1202$, Found 334.1193.

\section{7-(Benzo[d][1,3]dioxol-5-ylmethyl)-5,6-diphenyl-[1,3]dioxolo[4,5-g]quinolin-8(5H)-one} (6ah)

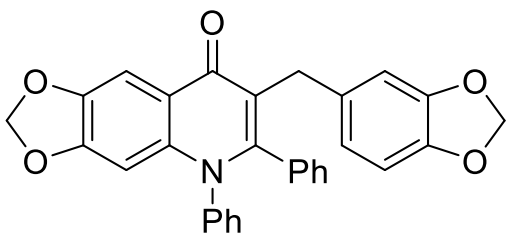

6ah
White solid (0.078 g, $82 \%$ yield, m.p. $\left.248.7-248.5^{\circ} \mathrm{C}\right) ; \mathrm{R}_{f}=0.5$ (silica, EtOAc:hexanes, 1:4); IR (film) $v_{\max } 3010,1628,1552$, 1489, 1472, 1318, $780 \mathrm{~cm}^{-1} ;{ }^{1} \mathrm{H}$ NMR $\left(400 \mathrm{MHz}, \mathrm{CDCl}_{3}\right) \delta=$ $7.90(\mathrm{~s}, 1 \mathrm{H}), 7.28-7.22(\mathrm{~m}, 3 \mathrm{H}), 7.13(\mathrm{dd}, J=5.1,1.9 \mathrm{~Hz}, 3 \mathrm{H})$,

$7.09-7.02(\mathrm{~m}, 2 \mathrm{H}), 6.97-6.90(\mathrm{~m}, 2 \mathrm{H}), 6.60-6.52(\mathrm{~m}, 2 \mathrm{H}), 6.37(\mathrm{dd}, J=8.0,1.7 \mathrm{~Hz}, 1 \mathrm{H})$, $6.11(\mathrm{~s}, 1 \mathrm{H}), 5.99(\mathrm{~s}, 2 \mathrm{H}), 5.83(\mathrm{~s}, 2 \mathrm{H}), 3.67(\mathrm{~s}, 2 \mathrm{H}) ;{ }^{13} \mathrm{C} \mathrm{NMR}\left(101 \mathrm{MHz}, \mathrm{CDCl}_{3}\right) \delta=176.0$, 151.3, 150.6, 147.0, 145.2, 145.1, 139.9, 139.1, 135.4, 134.2, 129.9, 129.7, 129.3, 128.7, 128.2, 127.7, 121.2, 120.9, 120.6, 109.1, 107.5, 103.4, 101.7, 100.4, 97.2, 32.1 ppm; HRMS (m/z): [M $+\mathrm{Na}]^{+}$calcd for $\mathrm{C}_{30} \mathrm{H}_{21} \mathrm{NO}_{5} \mathrm{Na}^{+} 498.1311$, Found 498.1300 .

7-(Benzo[d][1,3]dioxol-5-ylmethyl)-5-phenyl-6-(thiophen-2-yl)-[1,3]dioxolo[4,5-g]quinolin$8(5 \mathrm{H})$-one (6ai)

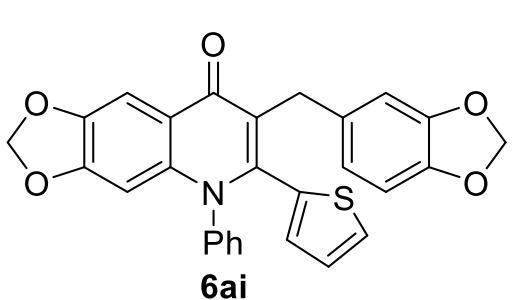

6ai
Colorless oil $\left(0.066 \mathrm{~g}, 69 \%\right.$ yield); $\mathrm{R}_{f}=0.5$ (silica, EtOAc:hexanes, 1:4); IR (film) $v_{\max } 2998,1624,1550,1478$, $1410,1312,740 \mathrm{~cm}^{-1} ;{ }^{1} \mathrm{H}$ NMR $\left(400 \mathrm{MHz}, \mathrm{CDCl}_{3}\right) \delta=7.86(\mathrm{~s}$, $1 \mathrm{H}), 7.33(\mathrm{~d}, J=6.2 \mathrm{~Hz}, 3 \mathrm{H}), 7.22(\mathrm{~d}, J=5.1 \mathrm{~Hz}, 1 \mathrm{H}), 7.14(\mathrm{~d}, J$ $=6.8 \mathrm{~Hz}, 2 \mathrm{H}), 6.78(\mathrm{t}, J=4.3 \mathrm{~Hz}, 1 \mathrm{H}), 6.65(\mathrm{~d}, J=4.8 \mathrm{~Hz}, 2 \mathrm{H}), 6.61(\mathrm{~d}, J=7.9 \mathrm{~Hz}, 1 \mathrm{H}), 6.50(\mathrm{~d}$, $J=8.0 \mathrm{~Hz}, 1 \mathrm{H}), 6.11(\mathrm{~s}, 1 \mathrm{H}), 6.00(\mathrm{~s}, 2 \mathrm{H}), 5.85(\mathrm{~s}, 2 \mathrm{H}), 3.79(\mathrm{~s}, 2 \mathrm{H}) ;{ }^{13} \mathrm{C} \mathrm{NMR}(101 \mathrm{MHz}$, $\left.\mathrm{CDCl}_{3}\right) \delta=175.7,151.6,147.1,145.4,145.2,143.5,139.8,139.5,135.2,134.1,130.4,129.8$ 
129.3, 128.9, 127.8, 126.3, 123.2, 121.1, 120.9, 109.0, 107.7, 103.3, 101.8, 100.5, 97.3, 32.6 ppm; HRMS (m/z): $[\mathrm{M}+\mathrm{Na}]^{+}$calcd for $\mathrm{C}_{28} \mathrm{H}_{19} \mathrm{NO}_{5} \mathrm{SNa}^{+}$504.0876, Found 504.0862.

7-(Benzo[d][1,3]dioxol-5-ylmethyl)-5-phenyl-[1,3]dioxolo[4,5-g]quinolin-8(5H)-one (6aj)

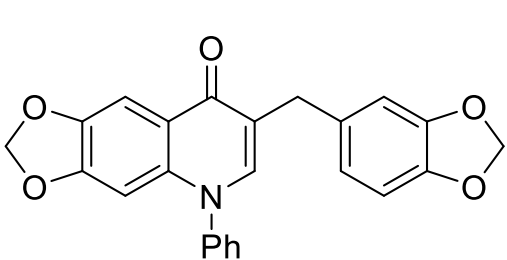

6aj

Colorless oil $\left(0.064 \mathrm{~g}, \quad 80 \%\right.$ yield); $R_{f}=0.5$ (silica, EtOAc:hexanes, 1:4); IR (film) $v_{\max } 3030,1628,1560,1474$, 1210, $736 \mathrm{~cm}^{-1} ;{ }^{1} \mathrm{H}$ NMR $\left(400 \mathrm{MHz}, \mathrm{CDCl}_{3}\right) \delta=7.84(\mathrm{~s}, 1 \mathrm{H})$, $7.59-7.51(\mathrm{~m}, 4 \mathrm{H}), 7.35-7.29(\mathrm{~m}, 2 \mathrm{H}), 6.85-6.67(\mathrm{~m}, 3 \mathrm{H})$, $6.33(\mathrm{~s}, 1 \mathrm{H}), 6.01(\mathrm{~s}, 2 \mathrm{H}), 5.89(\mathrm{~s}, 2 \mathrm{H}), 3.85(\mathrm{~s}, 2 \mathrm{H}) ;{ }^{13} \mathrm{C} \mathrm{NMR}\left(101 \mathrm{MHz}, \mathrm{CDCl}_{3}\right) \delta=175.8$, 151.4, 147.5, 145.7, 145.4, 141.6, 139.6, 138.1, 133.9, 130.2, 129.3, 127.5, 121.8, 121.7, 121.2 109.5, 108.1, 103.5, 101.8, 100.7, 96.2, $33.2 \mathrm{ppm}$; HRMS $(\mathrm{m} / \mathrm{z}):[\mathrm{M}+\mathrm{Na}]^{+}$calcd for $\mathrm{C}_{24} \mathrm{H}_{17} \mathrm{NO}_{5} \mathrm{Na}^{+} 422.0998$, Found 422.0990 .

\section{3-(3,4-diMethoxybenzyl)-6,7-dimethoxy-1,2-diphenylquinolin-4(1H)-one (6ak)}

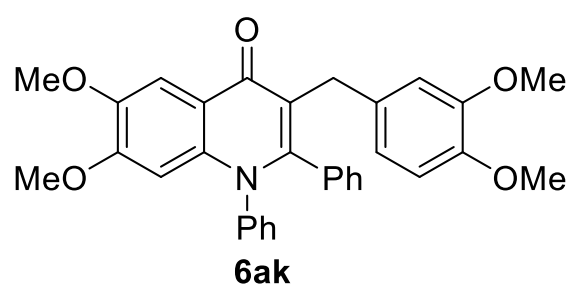

White solid $\left(0.083 \mathrm{~g}, 82 \%\right.$ yield, m.p. $\left.175.0-177.7^{\circ} \mathrm{C}\right) ; \mathrm{R}_{f}=$ 0.5 (silica, EtOAc:hexanes, 1:4); IR (film) $v_{\max } 3018,1624$, $1558,1472,1380,780 \mathrm{~cm}^{-1} ;{ }^{1} \mathrm{H} \mathrm{NMR}\left(400 \mathrm{MHz}, \mathrm{CDCl}_{3}\right) \delta=$ $7.93(\mathrm{~s}, 1 \mathrm{H}), 7.24(\mathrm{dd}, J=11.5,6.9 \mathrm{~Hz}, 3 \mathrm{H}), 7.17-7.10(\mathrm{~m}$,

3H), $7.06(\mathrm{~d}, J=7.4 \mathrm{~Hz}, 2 \mathrm{H}), 6.95(\mathrm{dd}, J=6.3,2.8 \mathrm{~Hz}, 2 \mathrm{H}), 6.61(\mathrm{q}, J=8.3 \mathrm{~Hz}, 2 \mathrm{H}), 6.49$ (s, $1 \mathrm{H}), 6.06(\mathrm{~s}, 1 \mathrm{H}), 4.00(\mathrm{~s}, 3 \mathrm{H}), 3.77(\mathrm{~s}, 3 \mathrm{H}), 3.73(\mathrm{~s}, 2 \mathrm{H}), 3.71(\mathrm{~s}, 3 \mathrm{H}), 3.57(\mathrm{~s}, 3 \mathrm{H}) ;{ }^{13} \mathrm{C} \mathrm{NMR}$ $\left(101 \mathrm{MHz}, \mathrm{CDCl}_{3}\right) \delta=176.1,152.7,150.4,148.1,146.8,146.7,139.7,137.5,134.4,130.0$, 129.9, 129.2, 128.6, 128.0, 127.6, 120.8, 120.4, 119.3, 112.2, 110.8, 105.6, 99.2, 56.1, 55.7, 55.6, 31.9 ppm; HRMS (m/z): [M + Na] ${ }^{+}$calcd for $\mathrm{C}_{32} \mathrm{H}_{29} \mathrm{NO}_{5} \mathrm{Na}^{+}$530.1937, Found 530.1924.

3-((2,3-diHydro-1H-inden-5-yl)methyl)-1,2-diphenyl-1,6,7,8-tetrahydro-4Hcyclopenta[g]quinolin-4-one (6al) 


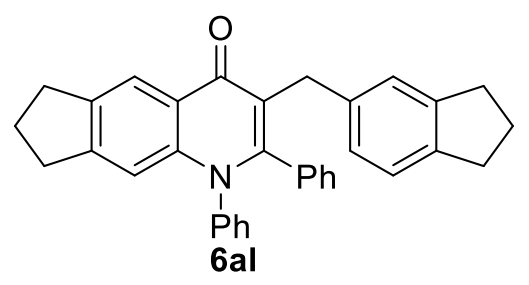

White solid $\left(0.073 \mathrm{~g}, 78 \%\right.$ yield, m.p. $\left.205.6-208.1^{\circ} \mathrm{C}\right) ; \mathrm{R}_{f}=0.5$ (silica, EtOAc:hexanes, 1:4); IR (film) $v_{\max } 2931,1626,1580$, 1476, 1387, 998, $791 \mathrm{~cm}^{-1} ;{ }^{1} \mathrm{H}$ NMR $\left(400 \mathrm{MHz}, \mathrm{CDCl}_{3}\right) \delta=$ $8.40(\mathrm{~s}, 1 \mathrm{H}), 7.27-7.23(\mathrm{~m}, 4 \mathrm{H}), 7.13(\mathrm{t}, J=3.6 \mathrm{~Hz}, 3 \mathrm{H}), 7.05$ (d, $J=6.6 \mathrm{~Hz}, 2 \mathrm{H}), 6.95(\mathrm{~d}, J=4.9 \mathrm{~Hz}, 2 \mathrm{H}), 6.88(\mathrm{~s}, 1 \mathrm{H}), 6.73(\mathrm{~d}, J=7.6 \mathrm{~Hz}, 1 \mathrm{H}), 6.54(\mathrm{~s}, 1 \mathrm{H})$, $3.75(\mathrm{~s}, 2 \mathrm{H}), 3.01(\mathrm{t}, J=7.3 \mathrm{~Hz}, 2 \mathrm{H}), 2.83-2.74(\mathrm{~m}, 6 \mathrm{H}), 2.11-2.03(\mathrm{~m}, 2 \mathrm{H}), 1.98(\mathrm{t}, J=7.4$ $\mathrm{Hz}, 2 \mathrm{H}) ;{ }^{13} \mathrm{C} N M R\left(101 \mathrm{MHz}, \mathrm{CDCl}_{3}\right) \delta=177.4,151.1,149.2,143.7,141.2,140.9,140.3,140.0$, 139.4, 134.5, 130.2, 129.8, 129.2, 128.3, 128.0, 127.6, 126.2, 124.5, 123.5, 121.1, 121.0, 112.9, 33.2, 32.7, 32.4, 32.2, 32.0, 25.8, 25.4 ppm; HRMS (m/z): $[\mathrm{M}+\mathrm{Na}]^{+}$calcd for $\mathrm{C}_{34} \mathrm{H}_{29} \mathrm{NONa}^{+}$ 490.2141, Found 490.2127.

\section{3-(3,4-diMethylbenzyl)-6,7-dimethyl-1,2-diphenylquinolin-4(1H)-one (6am)}

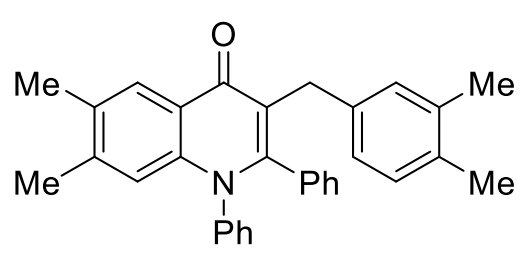

6am White solid (0.069 g, $78 \%$ yield, m.p. $\left.220.1-222.3^{\circ} \mathrm{C}\right) ; \mathrm{R}_{f}=0.5$ (silica, EtOAc:hexanes, 1:4); IR (film) $v_{\max }$ 2999, 1578, 1472, 1387, 996, $788 \mathrm{~cm}^{-1} ;{ }^{1} \mathrm{H}$ NMR $\left(400 \mathrm{MHz}, \mathrm{CDCl}_{3}\right) \delta=8.31(\mathrm{~s}$, $1 \mathrm{H}), 7.31-7.23(\mathrm{~m}, 3 \mathrm{H}), 7.18-7.09(\mathrm{~m}, 3 \mathrm{H}), 7.08-7.00(\mathrm{~m}$, 2H), $6.94(\mathrm{dt}, J=5.8,1.8 \mathrm{~Hz}, 2 \mathrm{H}), 6.87(\mathrm{~d}, J=7.6 \mathrm{~Hz}, 1 \mathrm{H}), 6.77-6.67(\mathrm{~m}, 2 \mathrm{H}), 6.47(\mathrm{~s}, 1 \mathrm{H})$, $3.72(\mathrm{~s}, 2 \mathrm{H}), 2.37(\mathrm{~s}, 3 \mathrm{H}), 2.19(\mathrm{~s}, 3 \mathrm{H}), 2.14(\mathrm{~s}, 3 \mathrm{H}), 2.11(\mathrm{~s}, 3 \mathrm{H}) ;{ }^{13} \mathrm{C} \mathrm{NMR}\left(101 \mathrm{MHz}, \mathrm{CDCl}_{3}\right) \delta$ $=177.1,151.1,141.4,140.4,139.8,139.0,135.6,134.4,133.2,132.6,130.2,130.0,129.9$, $129.1,129.0,128.4,128.0,127.6,126.3,125.9,123.4,121.2,117.9,31.9,20.5,19.6,19.2,19.2$ ppm; HRMS (m/z): [M + Na] $]^{+}$calcd for $\mathrm{C}_{32} \mathrm{H}_{29} \mathrm{NONa}^{+} 466.2141$, Found 466.2128.

\section{3-(3,4-diFluorobenzyl)-6,7-difluoro-1,2-diphenylquinolin-4(1H)-one (6an)}<smiles>O=c1c(Cc2ccc(F)c(F)c2)c(-c2ccccc2)n(-c2ccccc2)c2cc(F)c(F)cc12</smiles>

6an
White solid $\left(0.063 \mathrm{~g}, 69 \%\right.$ yield, m.p. $\left.153.0-155.7^{\circ} \mathrm{C}\right) ; \mathrm{R}_{f}=0.5$ (silica, EtOAc:hexanes, 1:4); IR (film) $v_{\max } 3020,1580,1472$, $1187,996,769 \mathrm{~cm}^{-1} ;{ }^{1} \mathrm{H} \mathrm{NMR}\left(400 \mathrm{MHz}, \mathrm{CDCl}_{3}\right) \delta=8.30$ (t, $J=$ 
$9.6 \mathrm{~Hz}, 1 \mathrm{H}), 7.33-7.26(\mathrm{~m}, 3 \mathrm{H}), 7.16(\mathrm{qd}, J=7.1,3.7 \mathrm{~Hz}, 3 \mathrm{H}), 7.08-7.01(\mathrm{~m}, 2 \mathrm{H}), 6.94-6.82$ $(\mathrm{m}, 3 \mathrm{H}), 6.73(\mathrm{ddd}, J=11.6,7.7,2.1 \mathrm{~Hz}, 1 \mathrm{H}), 6.66-6.62(\mathrm{~m}, 1 \mathrm{H}), 6.53(\mathrm{dd}, J=11.9,6.3 \mathrm{~Hz}$, 1H), $3.69(\mathrm{~s}, 2 \mathrm{H}) ;{ }^{13} \mathrm{C} \operatorname{NMR}\left(101 \mathrm{MHz}, \mathrm{CDCl}_{3}\right) \delta=174.8,153.3(\mathrm{~d}, J=15.0 \mathrm{~Hz}), 151.5,150.8(\mathrm{~d}$, $J=15.0 \mathrm{~Hz}), 150.0(\mathrm{~d}, J=15.0 \mathrm{~Hz}),, 148.8(\mathrm{~d}, J=14.0 \mathrm{~Hz}), 148.1(\mathrm{~d}, J=14.0 \mathrm{~Hz}), 148.1(\mathrm{~d}, J$ $=14.0 \mathrm{~Hz}), 147.5(\mathrm{~d}, J=12.1 \mathrm{~Hz}), 146.4(\mathrm{~d}, J=12.9 \mathrm{~Hz}), 145.6(\mathrm{~d}, J=15.0 \mathrm{~Hz}), 138.1,137.9$ (d, $J=9.8 \mathrm{~Hz}), 136.9(\mathrm{~m}), 132.6,128.7,128.4,128.2,127.7,127.1,123.3(\mathrm{~m}), 121.1(\mathrm{~m}), 119.6$, $116.2(\mathrm{~d}, J=17.4 \mathrm{~Hz}), 115.4(\mathrm{~d}, J=17.0 \mathrm{~Hz}), 112.9(\mathrm{~d}, J=18.1 \mathrm{~Hz}), 105.5(\mathrm{~d}, J=23.0 \mathrm{~Hz})$, 30.7 ppm; HRMS (m/z): $[\mathrm{M}+\mathrm{Na}]^{+}$calcd for $\mathrm{C}_{28} \mathrm{H}_{17} \mathrm{~F}_{4} \mathrm{NONa}^{+}$482.1138, Found 482.1124.

6-Methyl-3-(4-methylbenzyl)-1,2-diphenylquinolin-4(1H)-one (6ao)

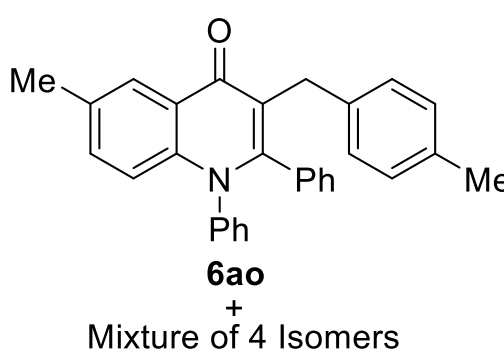

$(1: 1: 1: 1)$
Colorless oil $\left(0.070 \mathrm{~g}, 78 \%\right.$ yield); $\mathrm{R}_{f}=0.5$ (silica, EtOAc:hexanes, 1:4); IR (film) $\mathrm{v}_{\max }$ 3050, 2964, 2866, 1580, 1490, 1481, 1187, 996, $740 \mathrm{~cm}^{-1} ;{ }^{1} \mathrm{H}$ NMR (400 MHz, $\left.\mathrm{CDCl}_{3}\right)$ $\delta=8.48-8.44(\mathrm{~m}), 8.38-8.36(\mathrm{~m}), 7.29-7.21(\mathrm{~m}), 7.19-$ $7.10(m), 7.06-7.04(m), 7.01-6.86(m), 6.79-6.74(m)$,

$6.65-6.62(\mathrm{~m}), 6.47-6.5(\mathrm{~m}), 3.77(\mathrm{~s}), 3.76(\mathrm{~s}), 3.75(\mathrm{~s}), 3.73(\mathrm{~s}), 2.46(\mathrm{~d}), 2.30(\mathrm{~d}), 2.24(\mathrm{~s})$, $2.20(\mathrm{~s}) ;{ }^{13} \mathrm{C}$ NMR $\left(101 \mathrm{MHz}, \mathrm{CDCl}_{3}\right) \delta=177.3,177.2,151.6,151.5,142.1,142.0,141.4,140.0$, 139.6, 138.5, 138.4, 137.1, 134.6, 134.3, 134.3, 134.2, 133.1, 132.9, 130.1, 130.1, 129.7, 129.3, 129.3, 129.2, 128.4, 128.4, 128.1, 127.7, 127.6, 126.5, 126.5, 126.5, 126.0, 125.8, 125.8, 125.5, $125.0,124.9,123.0,121.4,121.3,121.3,121.2,117.8,117.4,32.2,32.2,32.0,32.0,22.0,21.3$ 20.9, 20.8 ppm; HRMS (m/z): [M + Na] ${ }^{+}$calcd for $\mathrm{C}_{30} \mathrm{H}_{25} \mathrm{NONa}^{+}$438.1828, Found 438.1821. 


\section{Control and cross-over experiments}

\section{a) One-pot cascade reactions carried out in $\mathrm{CD}_{3} \mathrm{CN}$}

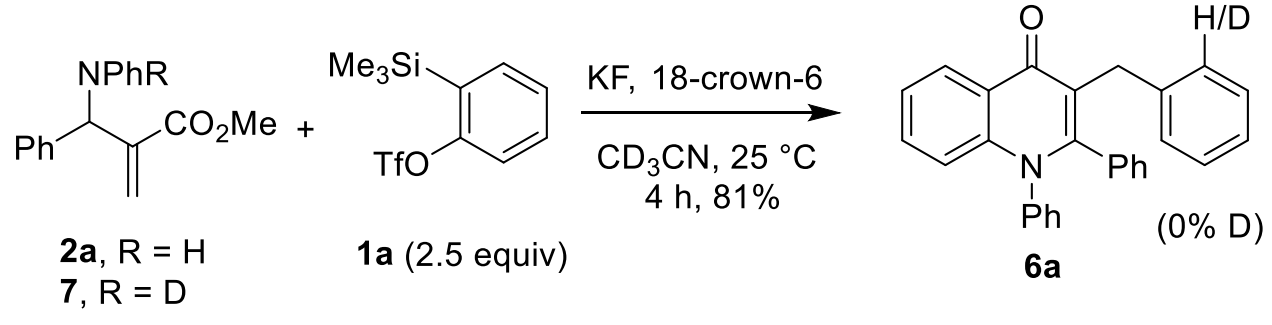

To a flame-dried screw-capped test tube equipped with a magnetic stir bar was added the 18crown-6 (131 mg, $0.5 \mathrm{mmol}, 5.0$ equiv) and $\mathrm{KF}$ (30 mg, $0.5 \mathrm{mmol}, 5.0$ equiv) under argon atmosphere, then $\mathrm{AMBH}$ adduct $\mathbf{2 a} / \mathbf{7}(0.10 \mathrm{mmol}, 1.0$ equiv) was added and the screw-capped tube was evacuated and backfilled with argon. The mixture was dissolved in $\mathrm{CD}_{3} \mathrm{CN}(1.0 \mathrm{~mL})$ under argon atmosphere. The resultant reaction mixture was kept stirring at room temperature for five minutes. To the stirring solution was added $\beta$-trimethylsilyltriflate $1 \mathrm{a}(75 \mathrm{mg}, 0.25 \mathrm{mmol}$, 2.5 equiv) dropwise, further allowed to stir for $4 \mathrm{~h}$. Then the reaction quenched and the solvent was evaporated. Subsequently, the crude residue was purified by flash column chromatography on silica gel to afford 4-quinolone $6 a$ a white solid (32 mg, 81\%).

\section{b) Ene reaction carried out in $\mathrm{CD}_{3} \mathrm{CN}$}<smiles>C=C1C(=O)c2ccccc2N(c2ccccc2)C1c1ccccc1</smiles>

$5 a$

(1 equiv)<smiles>C[Si](C)(C)c1ccccc1O</smiles>

$1 \mathrm{a}$ (1.2 equiv)

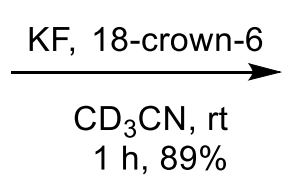

$1 \mathrm{~h}, 89 \%$<smiles>O=c1c(Cc2ccccc2)c(-c2ccccc2)n(-c2ccccc2)c2ccccc12</smiles>

$6 a$

To a flame-dried screw-capped test tube equipped with a magnetic stir bar was added the 18crown-6 (48 mg, $0.18 \mathrm{mmol}, 2.0$ equiv) and KF (11 mg, $0.18 \mathrm{mmol}, 2.0$ equiv) under argon 
atmosphere, then enone $5 \mathrm{a}$ ( $22 \mathrm{mg}, 0.07 \mathrm{mmol}, 1.0$ equiv) was added and the screw-capped tube was evacuated and backfilled with argon. The mixture was dissolved in $\mathrm{CD}_{3} \mathrm{CN}(1.0 \mathrm{~mL})$ under argon atmosphere. The resultant reaction mixture was kept stirring at room temperature for five minutes. To the stirring solution was added $\beta$-trimethylsilyltriflate $1 \mathrm{a}(27 \mathrm{mg}, 0.09 \mathrm{mmol}$, 1.2 equiv) dropwise, further allowed to stir for $1 \mathrm{~h}$. Then the reaction quenched and the solvent was evaporated. Subsequently, the crude residue was purified by flash column chromatography on silica gel to afford 4-quinolone $6 a$ a white solid (24 mg, 89\%).

The reaction of $\mathbf{2 a}$ or $\mathbf{7}$ with $\mathbf{1 a}$ carried out in $C D_{3} \mathrm{CN}$ resulted in the smooth formation of the corresponding protonated product 6 a in $81 \%$ (Scheme 7 of the manuscript). The no deuterium incorporation in the product sheds light on a concerted ene reaction. The reaction of $5 \mathbf{a}$ with 1 a carried out in $\mathrm{CD}_{3} \mathrm{CN}$ resulted in the smooth formation of the corresponding protonated product $6 \mathbf{a}$ in $89 \%$ (Scheme 7 of the manuscript). The no deuterium incorporation in the product sheds light on a concerted ene reaction.

The reaction of $\mathbf{2 a}, \mathbf{7}$ or $\mathbf{5 a}$ with $\mathbf{1 a}$ carried out in $C D_{3} C N$ resulted in the smooth formation of the corresponding protonated product 6a (Scheme 7 of the manuscript). The no deuterium incorporation in the product sheds light on a concerted ene reaction.

\section{c) Experiment for olefin isomerization}

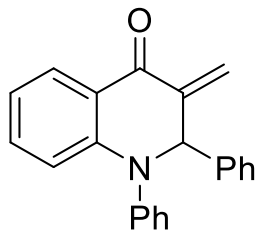

5 a

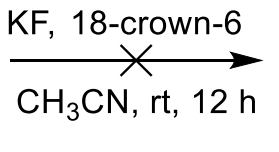

$\mathrm{CH}_{3} \mathrm{CN}, \mathrm{rt}, 12 \mathrm{~h}$

To a flame-dried screw-capped test tube equipped with a magnetic stir bar was added the 18crown-6 (40 mg, 0.15 mmol, 2.0 equiv) and KF (9 mg, $0.15 \mathrm{mmol}, 2.0$ equiv) under argon atmosphere, then enone $5 \mathbf{a}$ (22 $\mathrm{mg}, 0.07 \mathrm{mmol}, 1.0$ equiv) was added and the screw-capped 
tube was evacuated and backfilled with argon. The mixture was dissolved in $\mathrm{CH}_{3} \mathrm{CN}(3.0 \mathrm{~mL})$ under argon atmosphere. The resultant reaction mixture was kept stirring at room temperature for $12 \mathrm{~h}$. Compound 5a remained unchanged and olefin isomerization was not observed.

This result support that the second aryne addition preferentially follows the ene reaction rather than base mediated deprotonation and nucleophilic addition.

\section{d) Deuterium labeling experiment}<smiles>C=CC(=O)OCC(=O)OCC(=O)O</smiles>

SI-5
SI-6<smiles>[2H]C(O)(C(=C)C(=O)OC)c1ccccc1</smiles>

SI-7<smiles>[2H]C(C)(OC(C)=O)C(=C)C(=O)OC</smiles>

SI-8 $\mathrm{PhNH}_{2}$, DABCO THF, rt, $3 \mathrm{~h}$<smiles>[2H]c1ccccc1Cc1c(-c2ccccc2)n(-c2ccccc2)c2ccccc2c1=O</smiles>

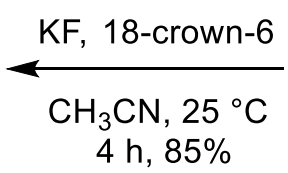

$4 \mathrm{~h}, 85 \%$<smiles>[2H]C(Nc1ccccc1)(C(=C)C(=O)OC)c1ccccc1</smiles>

2a-d

\section{Methyl 2-(hydroxy(phenyl)methyl-d)acrylate (SI-7)}<smiles>[2H]C(O)(C(=C)C(=O)OC)c1ccccc1</smiles>

SI-7

The $\mathrm{MBH}$ adduct SI-7 was prepared according to the literature procedure ${ }^{8}$ Pale yellow oil (88\% yield); $\mathrm{R}_{f}=0.3$ (silica, EtOAc:hexanes, 1:9); ${ }^{1} \mathrm{H}$ NMR $\left(400 \mathrm{MHz}, \mathrm{CDCl}_{3}\right) \delta=7.36-7.22(\mathrm{~m}, 5 \mathrm{H}), 6.30(\mathrm{~s}, 1 \mathrm{H}), 5.84(\mathrm{~s}, 1 \mathrm{H}), 3.68-$ $3.66(\mathrm{~m}, 3 \mathrm{H}) ;{ }^{13} \mathrm{C}$ NMR $\left(101 \mathrm{MHz}, \mathrm{CDCl}_{3}\right) \delta=166.5,141.8,141.1,128.2,127.6,126.5,125.8$, 125.7, $72.7-72.2(\mathrm{~m}), 51.7 \mathrm{ppm}$; $\operatorname{HRMS}(\mathrm{m} / \mathrm{z}):[\mathrm{M}+\mathrm{Na}]^{+}$calcd for $\mathrm{C}_{11} \mathrm{H}_{11} \mathrm{DO}_{3} \mathrm{Na}^{+} 216.0741$, Found 216.0741.

Methyl 2-(acetoxy(phenyl)methyl- $₫$ )acrylate (SI-8) 
The MBH acetate adduct SI-8 was prepared according to the literature<smiles>[2H]C(C)(OC(C)C)C(=C)C(=O)OC</smiles>

SI-8 procedure. ${ }^{8}$ Pale yellow oil $\left(94 \%\right.$ yield); $\mathrm{R}_{f}=0.5$ (silica, EtOAc:hexanes, 1:9); ${ }^{1} \mathrm{H}$ NMR $\left(400 \mathrm{MHz}, \mathrm{CDCl}_{3}\right) \delta=7.46-7.26(\mathrm{~m}, 5 \mathrm{H}), 6.40(\mathrm{~s}, 1 \mathrm{H}), 5.86$ (s, $1 \mathrm{H}), 3.70(\mathrm{~s}, 3 \mathrm{H}), 2.10(\mathrm{~s}, 3 \mathrm{H}) ;{ }^{13} \mathrm{C} \mathrm{NMR}\left(101 \mathrm{MHz}, \mathrm{CDCl}_{3}\right) \delta=169.3$, 165.3, 139.5, 137.6, 128.4, 128.3, 127.5, 125.7, 72.7, 51.9, 21.0 ppm; HRMS (m/z): [M + Na] ${ }^{+}$ calcd for $\mathrm{C}_{13} \mathrm{H}_{13} \mathrm{DO}_{4} \mathrm{Na}^{+} 258.0847$, Found 258.0846.

\section{Methyl 2-(phenyl(phenylamino)methyl- $d$ )acrylate (2a- $d$ )}

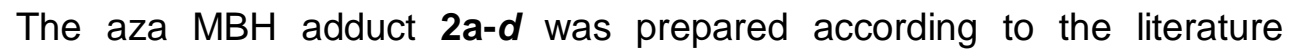<smiles>[2H]C(N)(C(=C)C(=O)OC)c1ccccc1</smiles>

2a-d procedure. ${ }^{8}$ Pale yellow oil $\left(86 \%\right.$ yield); $\mathrm{R}_{f}=0.5$ (silica, EtOAc:hexanes, 0.5:9.5); ${ }^{1} \mathrm{H}$ NMR $\left(400 \mathrm{MHz}, \mathrm{CDCl}_{3}\right) \delta=7.48-7.34(\mathrm{~m}, 5 \mathrm{H}), 7.27-7.20$ $(\mathrm{m}, 2 \mathrm{H}), 6.81-6.77(\mathrm{~m}, 1 \mathrm{H}), 6.68-6.63(\mathrm{~m}, 2 \mathrm{H}), 6.47(\mathrm{~s}, 1 \mathrm{H}), 6.04(\mathrm{~s}$, $1 \mathrm{H}), 4.26(\mathrm{~s}, 1 \mathrm{H}), 3.75(\mathrm{~s}, 3 \mathrm{H}) ;{ }^{13} \mathrm{C} \operatorname{NMR}\left(101 \mathrm{MHz}, \mathrm{CDCl}_{3}\right) \delta=166.5,146.5,140.4,139.9$, 129.0, 128.6, 127.6, 127.3, 126.0, 117.7, 113.3, 58.6, 58.4, 58.2, 51.7 ppm; HRMS (m/z): [M + $\mathrm{Na}]^{+}$calcd for $\mathrm{C}_{17} \mathrm{H}_{16} \mathrm{NO}_{2} \mathrm{Na}^{+}$291.1214, Found 291.1214.

\section{1,2-diPhenyl-3-((phenyl-2- $d)$ methyl)quinolin-4(1H)-one (6a- $d)$}

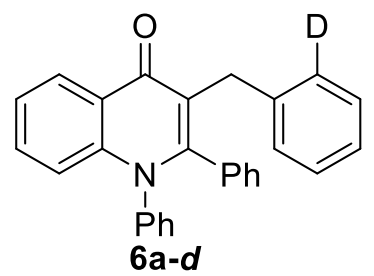

The deuterated 4-quinolone $\mathbf{6 a -} \boldsymbol{d}$ was prepared according to the general procedure of 6 . White solid (79\% yield, m.p. $\left.204.7-205.3^{\circ} \mathrm{C}\right) ; \mathrm{R}_{f}=0.4$ (silica, EtOAc:hexanes, 1:4); IR (film) $v_{\max }$ 2999, 1635, 1580, 1494, 1478, $698 \mathrm{~cm}^{-1} ;{ }^{1} \mathrm{H}$ NMR $\left(600 \mathrm{MHz}, \mathrm{CDCl}_{3}\right) \delta=8.57(\mathrm{~d}, J=8.0 \mathrm{~Hz}, 1 \mathrm{H}), 7.43-$ $7.40(\mathrm{~m}, 1 \mathrm{H}), 7.35$ - $7.33(\mathrm{~m}, 1 \mathrm{H}), 7.27-7.21(\mathrm{~m}, 3 \mathrm{H}), 7.18-7.02(\mathrm{~m}, 8 \mathrm{H}), 6.98(\mathrm{~d}, J=7.6 \mathrm{~Hz}$, $1 \mathrm{H}), 6.95-6.90(\mathrm{~m}, 2 \mathrm{H}), 6.73(\mathrm{~d}, \mathrm{~J}=8.5 \mathrm{~Hz}, 1 \mathrm{H}), 3.79(\mathrm{~s}, 2 \mathrm{H}) ;{ }^{13} \mathrm{C} \mathrm{NMR}\left(151 \mathrm{MHz}, \mathrm{CDCl}_{3}\right) \delta=$ $177.4,151.9,141.9,141.4,139.6,134.2,131.4,130.1,129.6,129.3,128.6,128.5,128.2,127.8$, 127.7, 127.6, 126.6, 125.3, 125.1, 123.3, 121.4, 117.9, 32.4 ppm; HRMS (m/z): [M + Na $]^{+}$calcd for $\mathrm{C}_{28} \mathrm{H}_{20} \mathrm{DNONa}^{+}$411.1578, Found 411.1574. 
Comparison of ${ }^{1} \mathrm{H}$ NMR of $\mathbf{6 a}$ with $\mathbf{6 a}-\boldsymbol{d}$ clearly supports the presence of deuterium on the aromatic moiety. Further HRMS data confirms the deuterium incorporation.
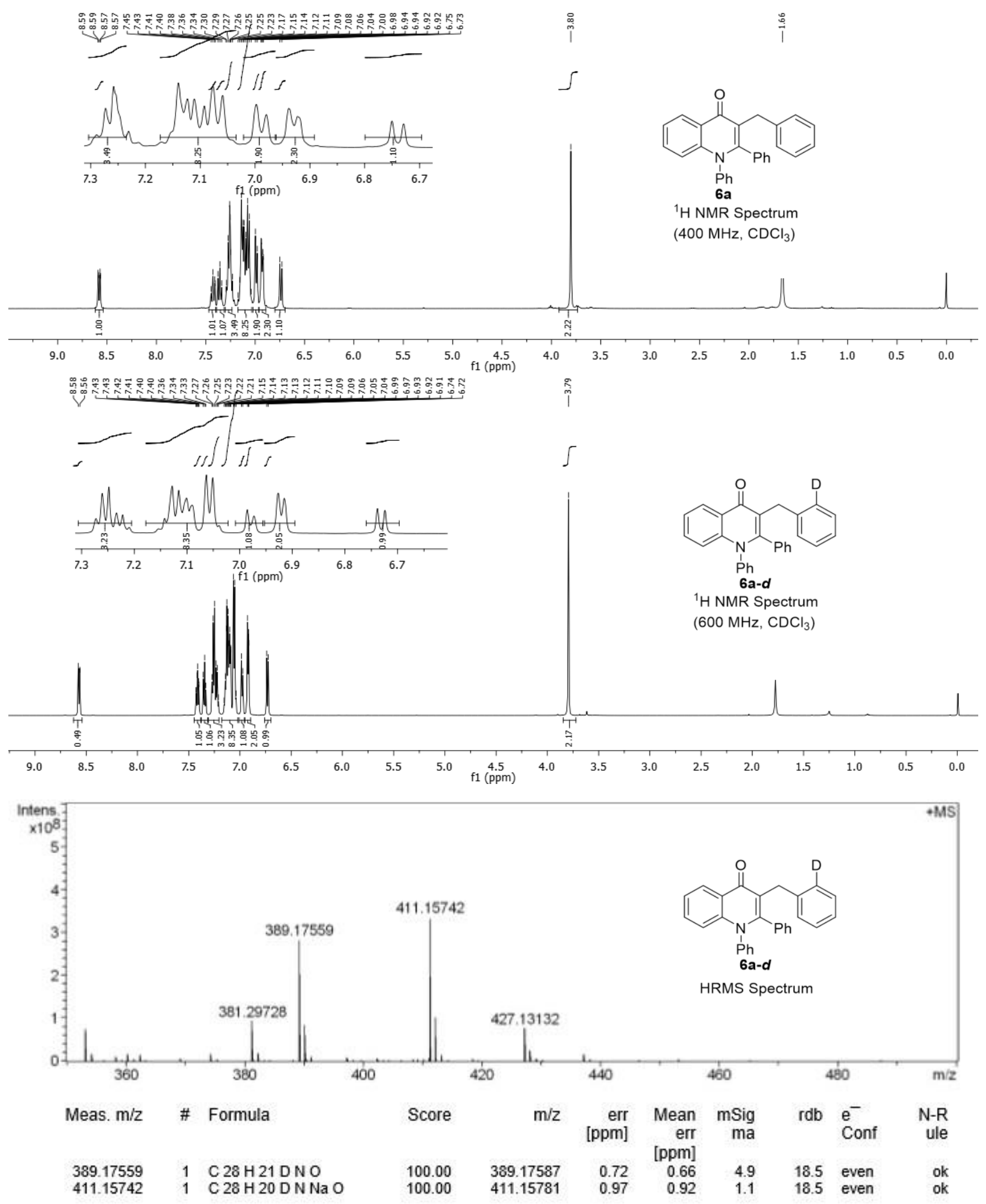


\section{e) Cross-over experiment}<smiles>COc1cc(OC)c(OC)cc1OC</smiles>

(1.2 equiv)<smiles>CCOc1cc(F)c(F)cc1[AsH2]C</smiles>

(1.2 equiv)

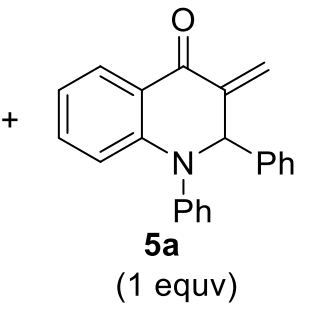

(1 equv)
KF, 18-crown-6

$\mathrm{CH}_{3} \mathrm{CN}, 25^{\circ} \mathrm{C}, 1 \mathrm{~h}$

$91 \%$

$(6 \mathrm{c}: 6 \mathrm{~g}=10: 1)$

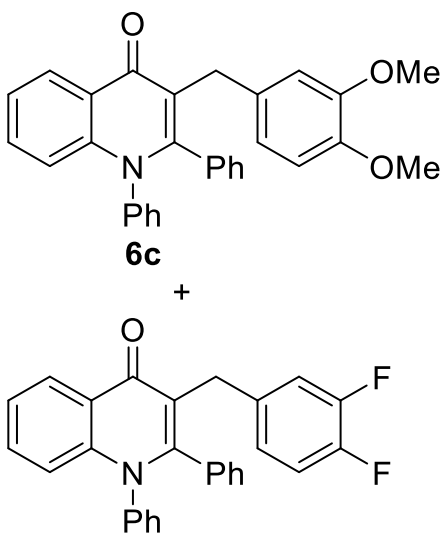

$6 \mathrm{~g}$

To a flame-dried screw-capped test tube equipped with a magnetic stir bar was added the 18crown-6 (95 mg, $0.36 \mathrm{mmol}, 2.0$ equiv) and $\mathrm{KF}$ (21 mg, $0.36 \mathrm{mmol}, 2.0$ equiv) under argon atmosphere, then enone $5 \mathrm{a}$ ( $23 \mathrm{mg}, 0.07 \mathrm{mmol}, 1.0$ equiv) was added and the screw-capped tube was evacuated and backfilled with argon. The mixture was dissolved in $\mathrm{CH}_{3} \mathrm{CN}(3.0 \mathrm{~mL})$ under argon atmosphere. The resultant reaction mixture was kept stirring at room temperature for five minutes. To the stirring solution was added 1:1 mixture of $1 \mathrm{c}(32 \mathrm{mg}, 0.09 \mathrm{mmol}, 1.2$ equiv) and $1 \mathrm{~g}$ (32 $\mathrm{mg}, 0.09 \mathrm{mmol}, 1.2$ equiv) dropwise, further allowed to stir for $1 \mathrm{~h}$. Then the reaction quenched and the solvent was evaporated. Subsequently, the crude residue was purified by flash column chromatography on silica gel to afford 4-quinolone $6 \mathrm{c}(55 \mathrm{mg})$ and $\mathbf{6 g}$ (5 mg).

Electron rich aryne-enophile $1 \mathrm{c}$ reacted almost 10 times faster than the electron deficient aryne 1g. This also indicates that reaction proceeds through inverse-electron-demand ene reaction.

\section{Scale-up experiment}

To a solution of AMBH adduct 2a $(1.0 \mathrm{~g}, 3.7 \mathrm{mmol}), \mathrm{KF}(1.1 \mathrm{~g}, 18.6 \mathrm{mmol}), 18-\mathrm{crown}-6(4.9 \mathrm{~g}$, $18.6 \mathrm{mmol})$ in anhydrous acetonitrile $(20 \mathrm{~mL})$ was added 2-(trimethylsilyl)-aryl triflate $1 \mathrm{a}(2.3 \mathrm{~mL}$, 
$9.3 \mathrm{mmol})$. The mixture was stirred for $4 \mathrm{~h}$ at room temperature under an argon atmosphere. The reaction mixture was then evaporated and resulting solid was washed with hexane $(20 \mathrm{~mL}$ $x 5)$ to give pure $6 a$ in $79 \%$ yield $(1.14 \mathrm{~g})$, thus indicating that this transformation is easy to scale up to gram scale without loss of efficiency.<smiles>C=C(OC)C(Nc1ccccc1)c1ccccc1</smiles>

$2 \mathbf{a}$<smiles>CCOc1ccccc1[SiH2]C</smiles>

$1 \mathbf{a}$

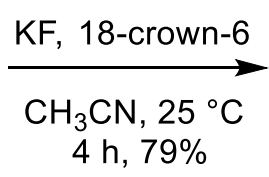

$4 \mathrm{~h}, 79 \%$<smiles>O=c1c(Cc2ccccc2)c(-c2ccccc2)n(-c2ccccc2)c2ccccc12</smiles>

$6 \mathbf{a}$

\section{Reaction with internal olefin SI-9}<smiles>CCOC(=O)/C(C)=C(\Nc1ccccc1)c1ccccc1</smiles>

SI-9<smiles>CCOc1ccccc1C</smiles>

1a

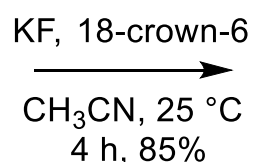

$4 \mathrm{~h}, 85 \%$<smiles>CCOC(=O)C(C)(C(=N)c1ccccc1)c1ccccc1</smiles>

SI-10

To check the generality of this methodology with an internal olefin, $\beta$-amino- $\alpha$-methyl unsaturated ester $\mathbf{S I - \mathbf { 9 } ^ { 1 4 }}$ was subjected under optimized condition with aryne $\mathbf{1 a}$, this reaction furnished the C-arylated ester SI-10 in $85 \%$ yield. ${ }^{15}$

To a flame-dried screw-capped test tube equipped with a magnetic stir bar was added the 18crown-6 (129 mg, $0.45 \mathrm{mmol}, 2.5$ equiv) and KF (28 mg, $0.45 \mathrm{mmol}, 2.5$ equiv) under argon atmosphere, then compound SI-9 (50 mg, $0.18 \mathrm{mmol}, 1.0$ equiv) was added and the screwcapped tube was evacuated and backfilled with argon. The mixture was dissolved in $\mathrm{CH}_{3} \mathrm{CN}$ $(2.0 \mathrm{~mL})$ under argon atmosphere. The resultant reaction mixture was kept stirring at room temperature for five minutes. To the stirring solution was added $\beta$-trimethylsilyltriflate $1 \mathrm{a}$ (75 mg, $0.25 \mathrm{mmol}, 1.4$ equiv) dropwise, further allowed to stir for $4 \mathrm{~h}$. Then the reaction quenched and the solvent was evaporated. Subsequently, the crude residue was purified by flash column 
chromatography on silica gel to afford C-arylated product SI-10 as yellow liquid (54 mg, 85\%). $\mathrm{R}_{f}=0.5$ (silica, EtOAc:hexanes, 0.5:9.5); ${ }^{1} \mathrm{H}$ NMR $\left(400 \mathrm{MHz}, \mathrm{CDCl}_{3}\right) \delta=7.62-7.57(\mathrm{~m}, 2 \mathrm{H})$, $7.40(\mathrm{dd}, J=8.4,6.4 \mathrm{~Hz}, 2 \mathrm{H}), 7.37-7.33(\mathrm{~m}, 1 \mathrm{H}), 7.12$ - $7.06(\mathrm{~m}, 3 \mathrm{H}), 7.04-6.99(\mathrm{~m}, 2 \mathrm{H}), 6.91$ - $6.85(\mathrm{~m}, 1 \mathrm{H}), 6.70-6.63(\mathrm{~m}, 4 \mathrm{H}), 4.30-4.21(\mathrm{~m}, 2 \mathrm{H}), 1.87(\mathrm{~s}, 3 \mathrm{H}), 1.30$ (t, J = $7.1 \mathrm{~Hz}, 3 \mathrm{H})$; ${ }^{13} \mathrm{C}$ NMR $\left(101 \mathrm{MHz}, \mathrm{CDCl}_{3}\right) \delta=173.4,172.5,150.0,139.4,135.6,128.5,128.3,128.2,128.0$, 127.9, 127.3, 127.3, 123.1, 120.4, 61.2, 60.6, 22.8, $14.0 \mathrm{ppm} ; \mathrm{HRMS}(\mathrm{m} / \mathrm{z}):[\mathrm{M}+\mathrm{Na}]^{+}$calcd for $\mathrm{C}_{24} \mathrm{H}_{23} \mathrm{NO}_{2} \mathrm{Na}^{+} 380.1621$, Found 380.1618 . 


\section{References}

1 (a) Sato, Y.; Tamura, T.; Kinbara, A.; Morib, M. Adv. Synth. Catal. 2007, 349, 647. (b) Pena, D.; Cobas, A.; Pérez, D.; Guitián, E. Synthesis 2002, 1454.

2 (a) Mphahlele, M. J. J. Heterocycl. Chem. 2010, 47, 1. (b) Kouznetsov, V. V.; Mendez, L. Y. V.; Gomez, C. M. M. Curr. Org. Chem. 2005, 9, 141.

3 (a) Gould, R. G.; Jacobs, W. A. J. Am. Chem. Soc. 1939, 61, 2890. (b) Heindel, N. D.; Bechara, I. S.; Kennewell, P. D.; Molnar, J.; Ohnmacht, C. J.; Lemke, S. M.; Lemke, T. F. J. Org. Chem. 1968, $11,1218$.

4 (a) Niementowski, S. Ber. Dtsch. Chem. Soc. 1894, 27, 1394. (b) Alexandre, F. R.; Berecibar, A.; Besson, T. Tetrahedron Lett. 2002, 43, 3911.

5 (a) Camps, R. Chem. Ber. 1899, 32, 3228. (b) Ding, D.; Li, X.; Wang, X.; Du, Y.; Shen, J. Tetrahedron Lett. 2006, 47, 6997. (c) Jones, C. P.; Anderson, K. W.; Buchwald, S. L. J. Org. Chem. 2007, 72, 7968. (d) Huang, J.; Chen, Y.; King, A. O.; Dilmeghani, M.; Larsen, R. D.; Faul, M. M. Org. Lett. 2008, 10, 2609.

6 (a) Liu, Q. L.; Li, Q. L.; Fei, X. D.; Zhu, Y. M. ACS Comb. Sci. 2011, 13, 19. (b) Hu, W.; Lin, J. P.; Song, L. R.; Long, Y. Q. Org. Lett. 2015, 17, 1268.

7 (a) Torii, S.; Okumoto, H.; Xu, L. H. Tetrahedron Lett. 1991, 32, 237. (b) Seppanen, O.; Muuronen, M.; Helaja, J. Eur. J. Org. Chem. 2014, 4044. (c) Zhao, T. K.; Xu, B. Org. Lett. 2010, 12, 212. (d) Bernini, R.; Cacchi, S.; Fabrizi, G.; Sferrazza, A. Synthesis-Stuttgart 2009, 1209. (e) Fei, X. D.; Zhou, Z.; Li, W.; Zhu, Y. M.; Shen, J. K. Eur. J. Org. Chem. 2012, 3001. (f) Shao, J.; Huang, X. M.; Hong, X. H.; Liu, B. X.; Xu, B. Synthesis-Stuttgart 2012, 44, 1798. (g) laroshenko, V. O.; Mkrtchyan, S.; Villinger, A. Synthesis-Stuttgart 2013, 45, 205. (h) Okamoto, N.; Takeda, K.; Ishikura, M.; Yanada, R. J. Org. Chem. 2011, 76, 9139.

8 (a) Nag, S.; Bhowmik, S.; Gauniyal, H. M.; Batra, S. Eur. J. Org. Chem. 2010, 4705. (b) Ko, S. H.; Lee, K-J. J. Heterocyclic Chem. 2004, 41, 613.

9 Lee, C. G.; Lee, K. Y.; Lee, S.; Kim, J. N. Tetrahedron, 2005, 61, 1493.

10 Ge, S. Q.; Hua, Y. Y.; Xia, M. Ultrason. Sonochem., 2009, 16, 743.

11 Liu, J.; Han, Z.; Wang, X.; Wang, Z.; Ding, K. J. Am. Chem. Soc., 2015, 137, 15346.

12 Chen, H. Y.; Patkar, L. N.; Ueng, S. H.; Lin, C. C.; Lee, A. S. Synlett 2005, 2035.

13 Murru, S.; Gallo, A. A.; Srivastava, R. S. J. Org. Chem., 2012, 77, 7119.

$14 \mathrm{He}, \mathrm{Z} . ; \mathrm{Li}, \mathrm{H} . ;$ Li, Z. J. Org. Chem., 2010, 75, 4636.

15 Ramtohul, Y. K.; Chartrand, A. Org. Lett. 2007, 9, 1029. 


\section{Spectra}
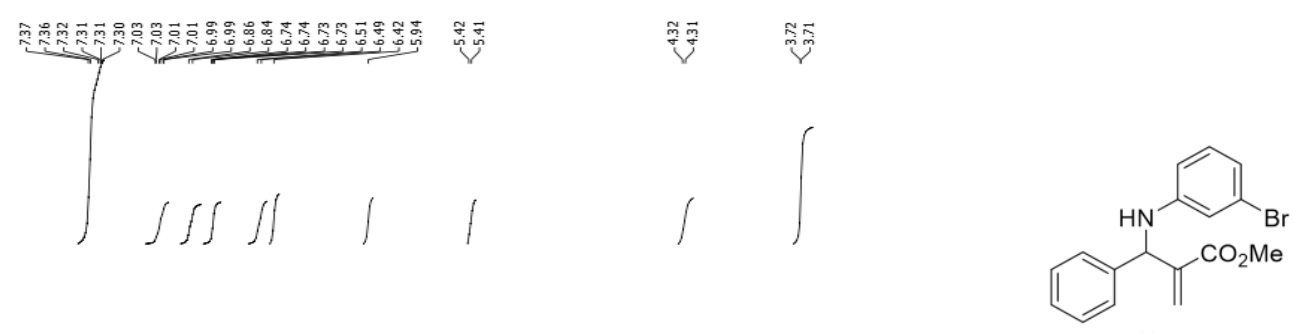

2h

${ }^{1} \mathrm{H}$ NMR Spectrum (400 MHz, $\mathrm{CDCl}_{3}$ )

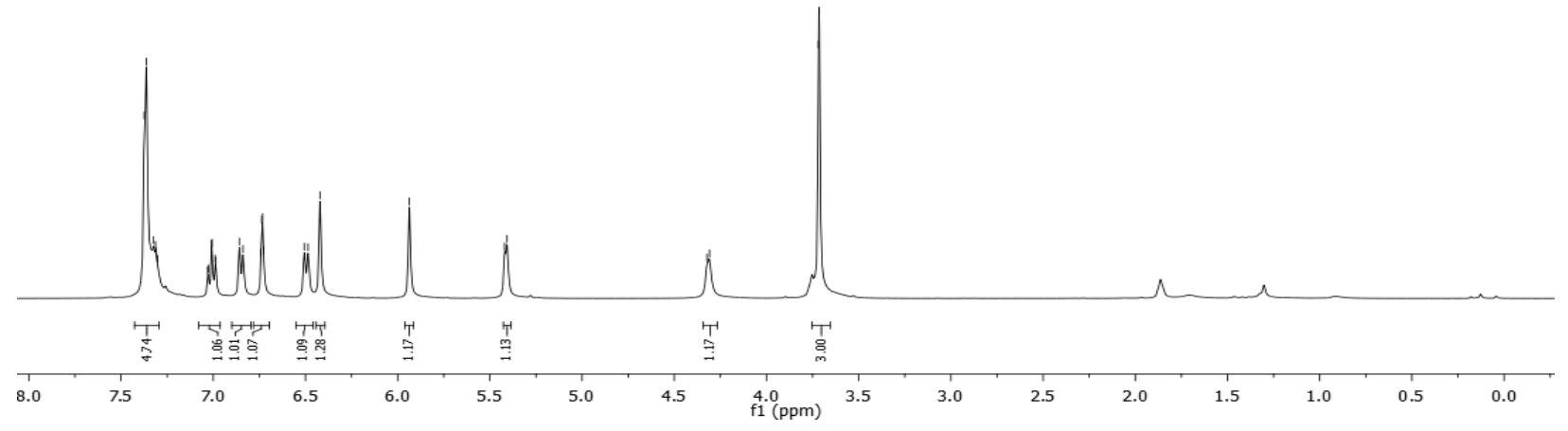

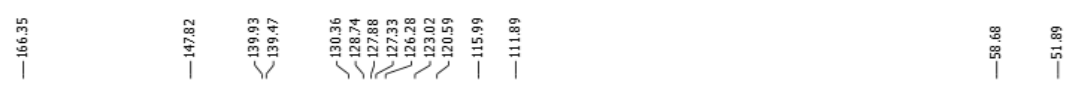
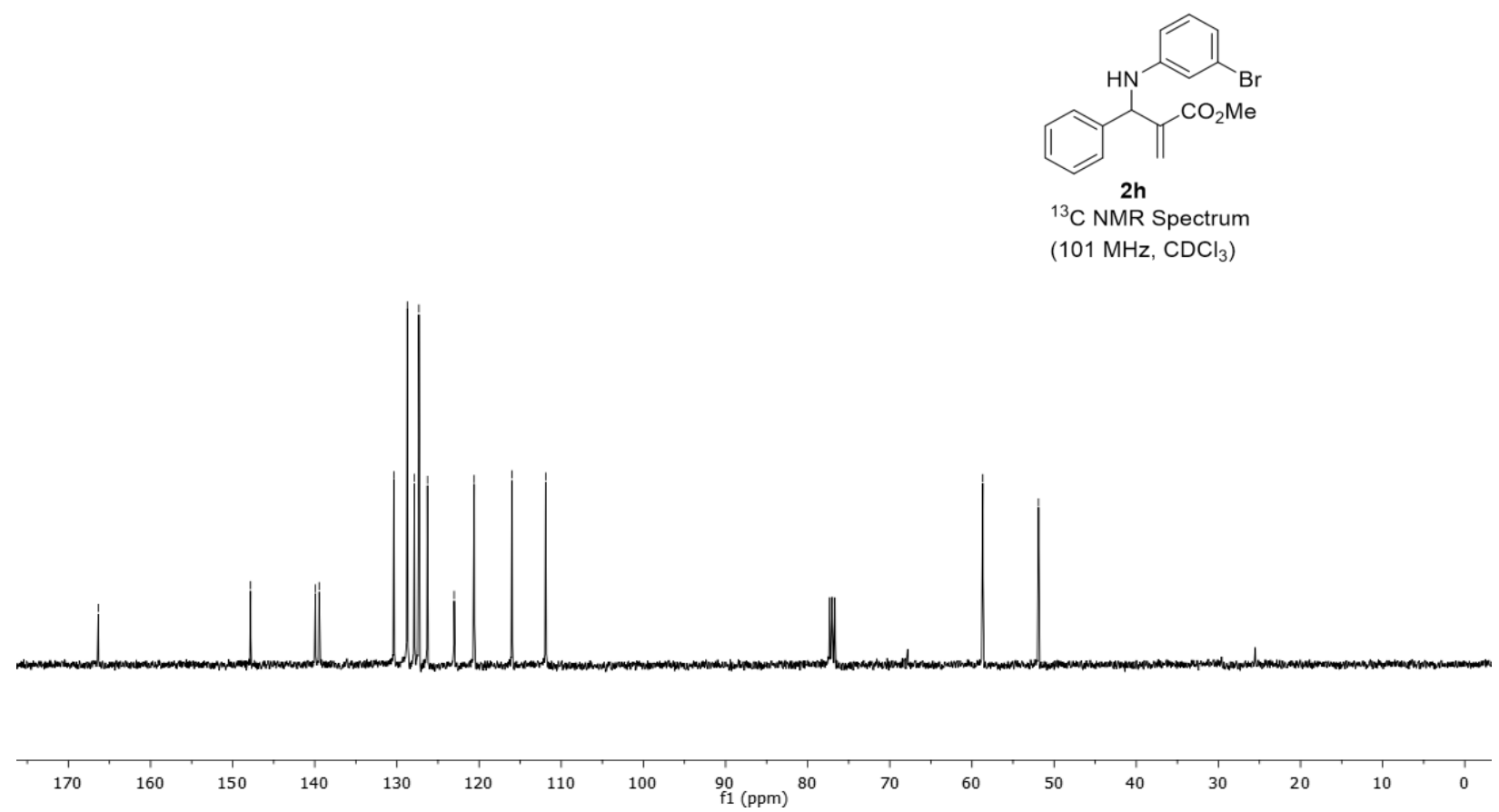


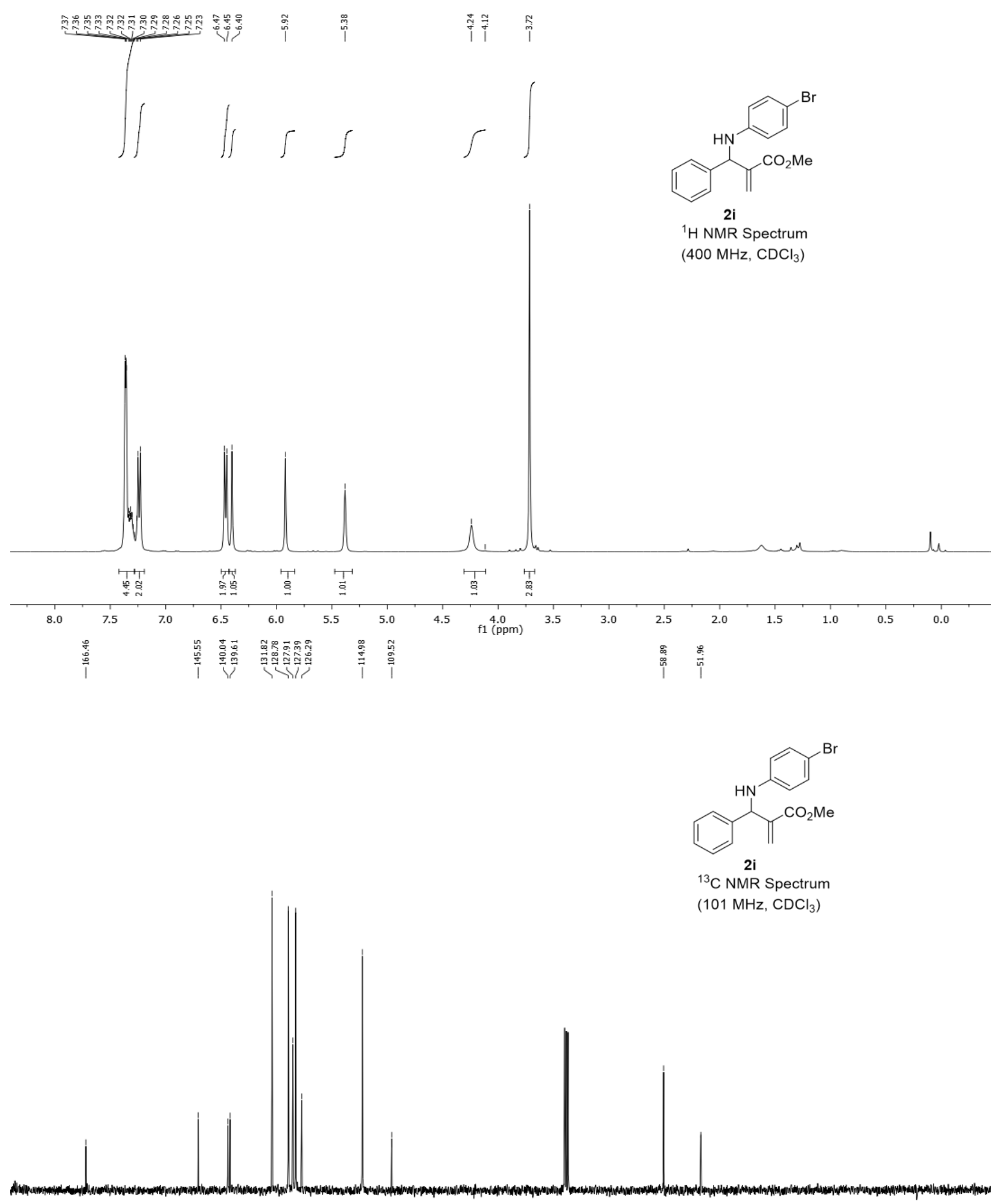

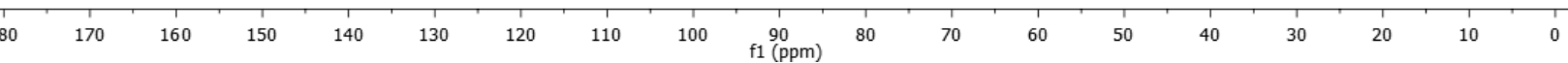



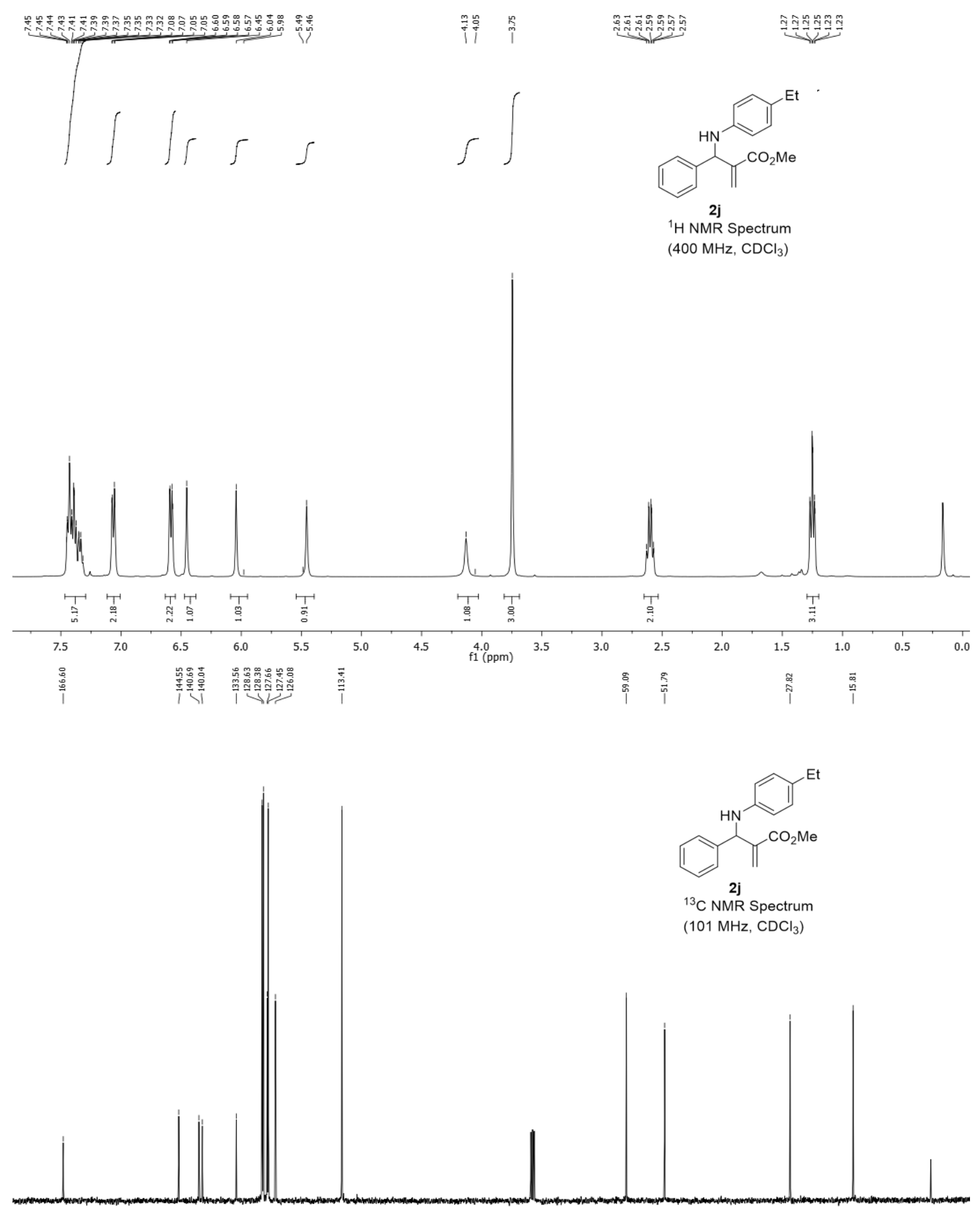

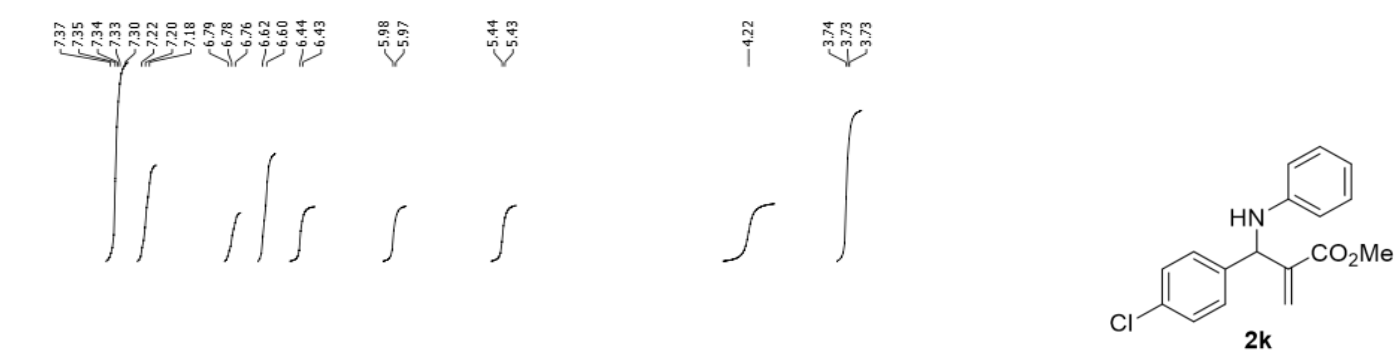

${ }^{1} \mathrm{H}$ NMR Spectrum

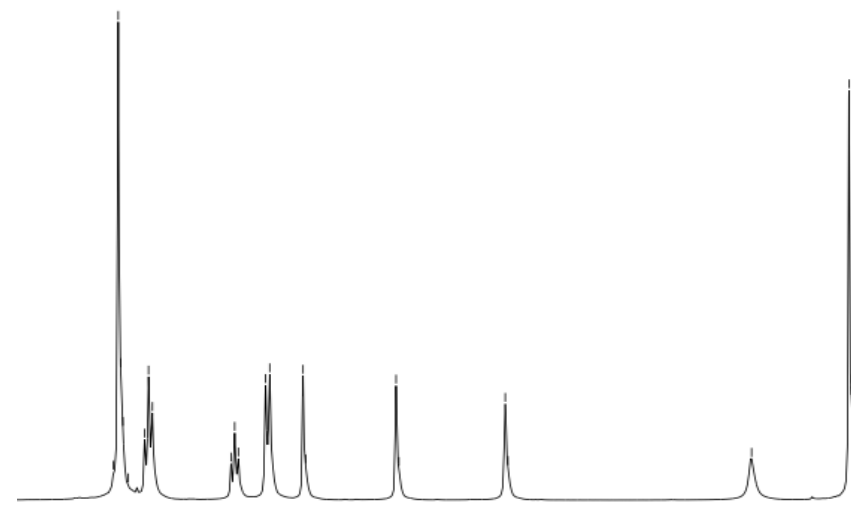

$\left(400 \mathrm{MHz}, \mathrm{CDCl}_{3}\right.$ )
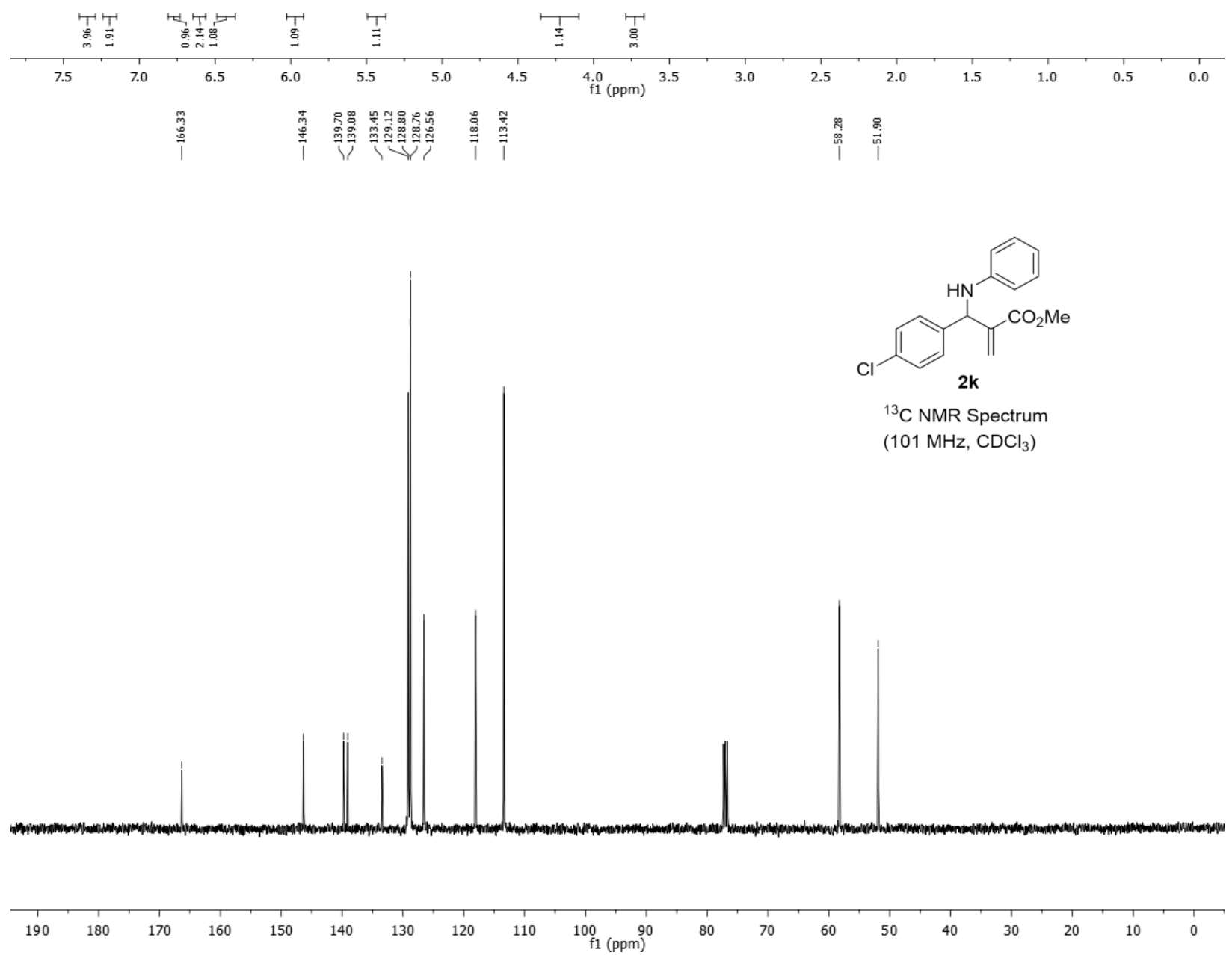

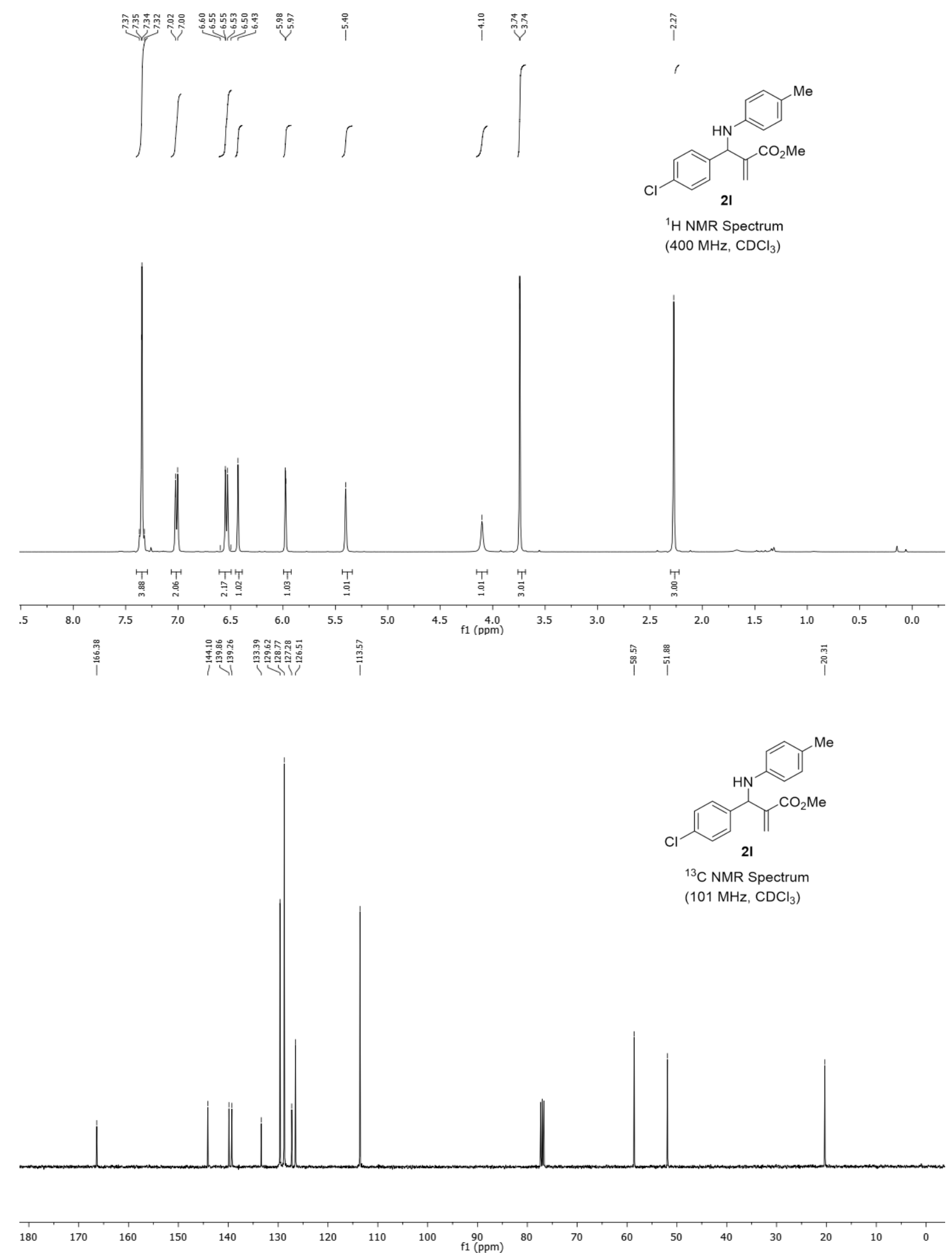

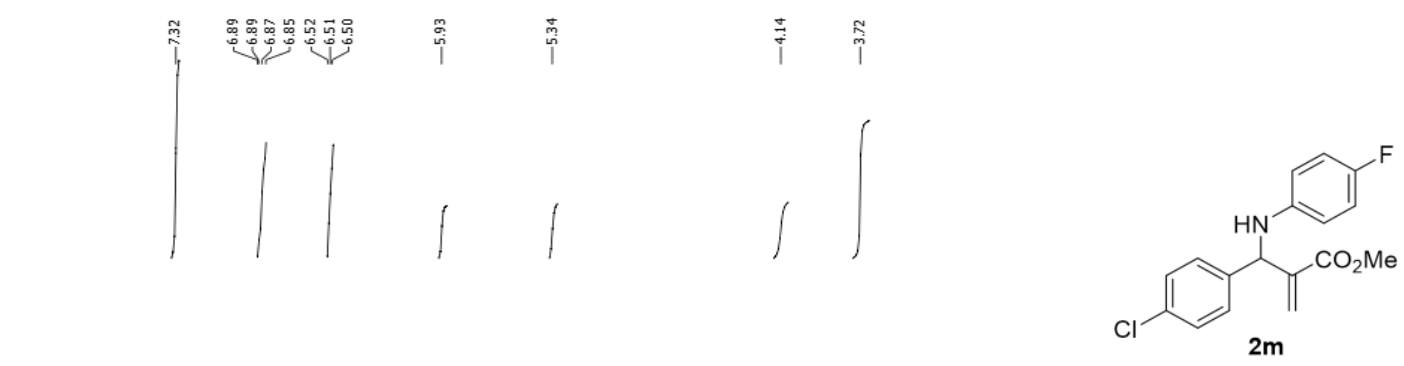

${ }^{1} \mathrm{H}$ NMR Spectrum (400 MHz, $\mathrm{CDCl}_{3}$ )
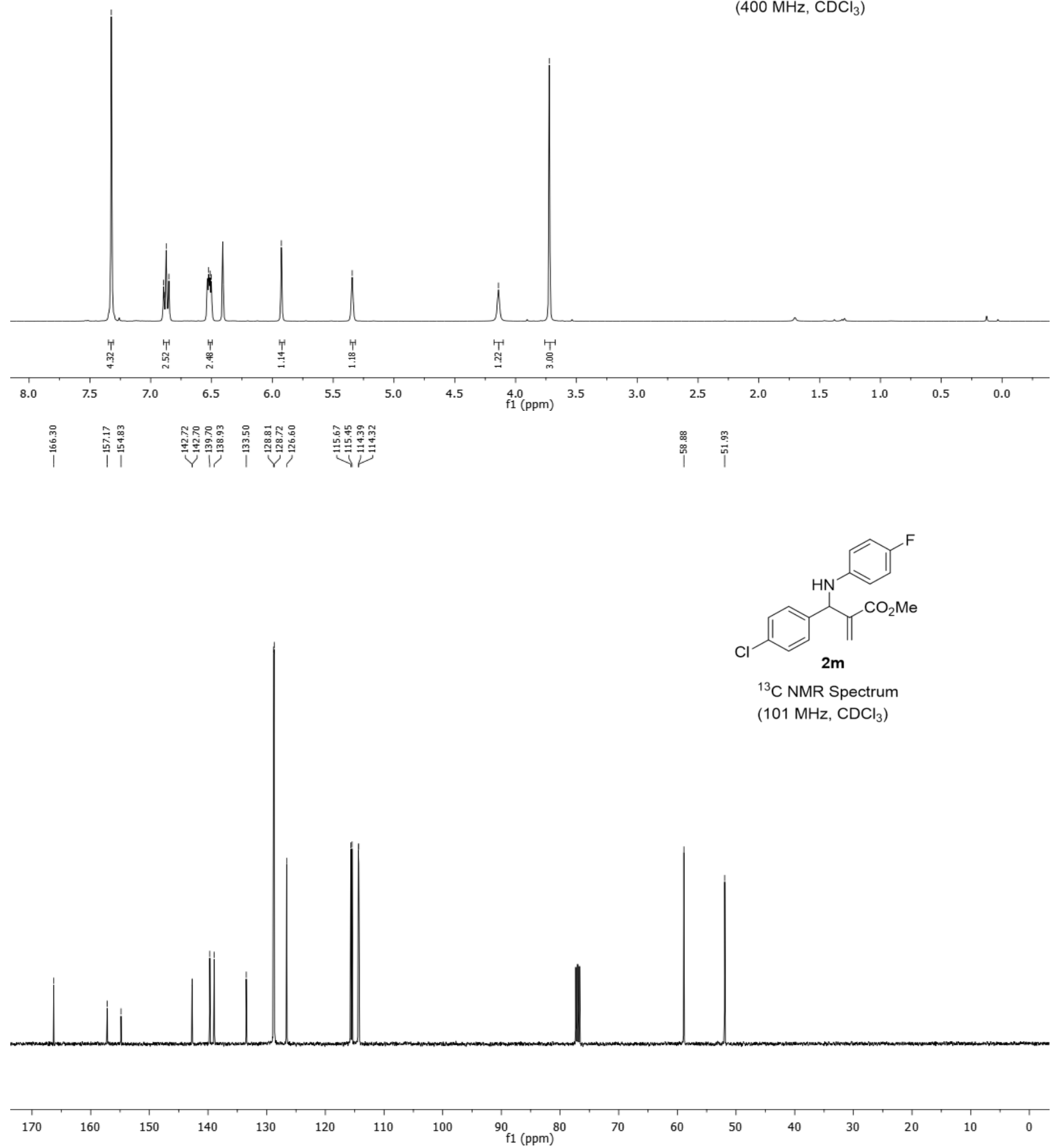

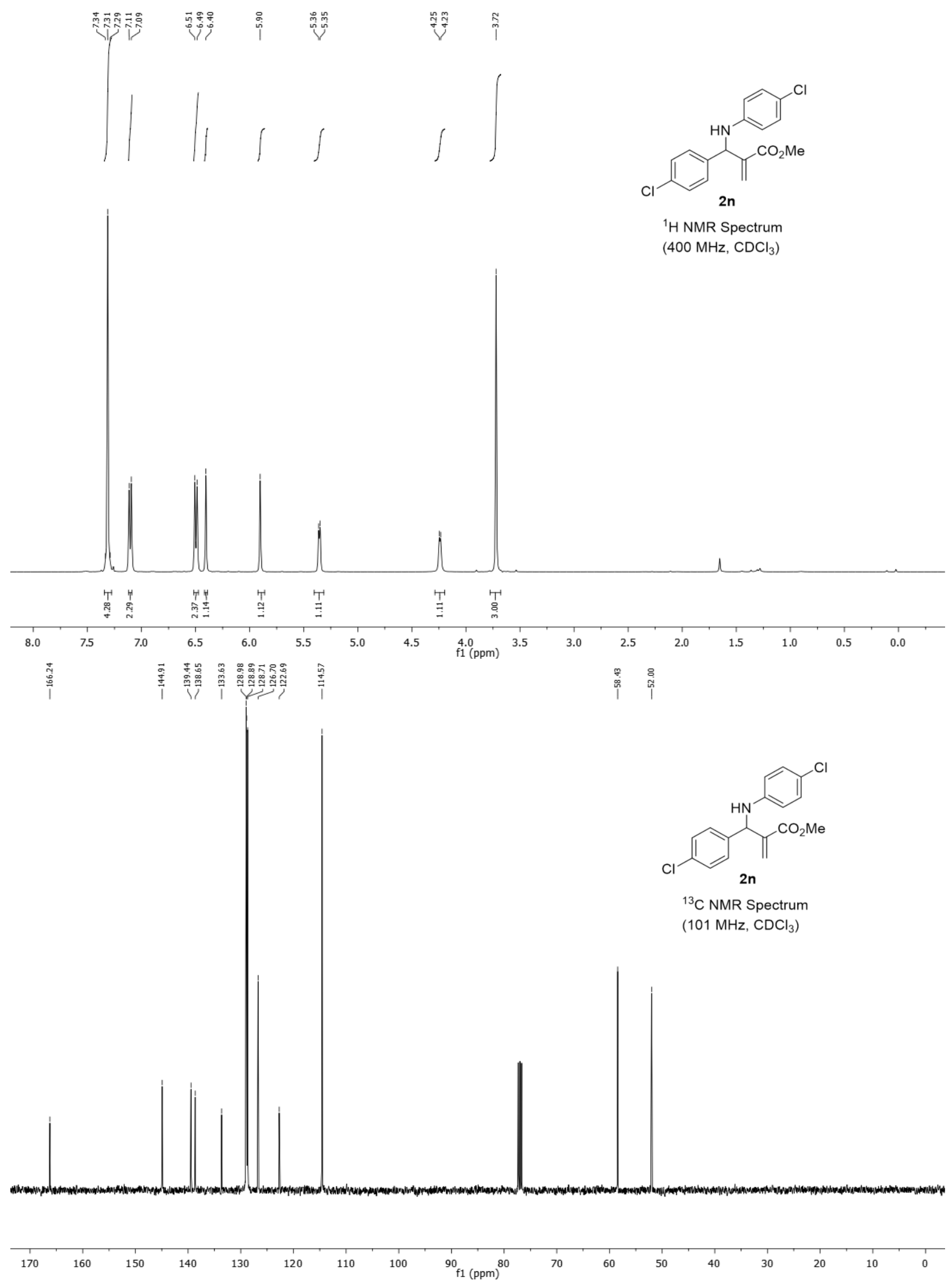


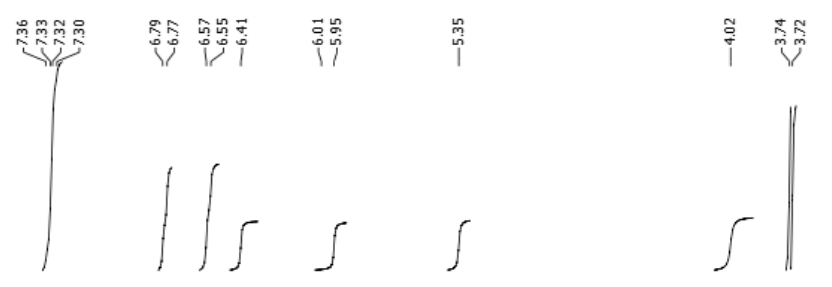

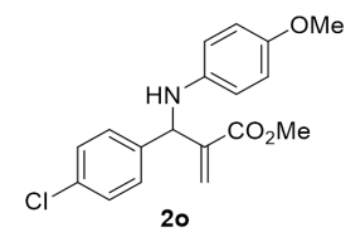

${ }^{1} \mathrm{H}$ NMR Spectrum (400 MHz, $\mathrm{CDCl}_{3}$ )
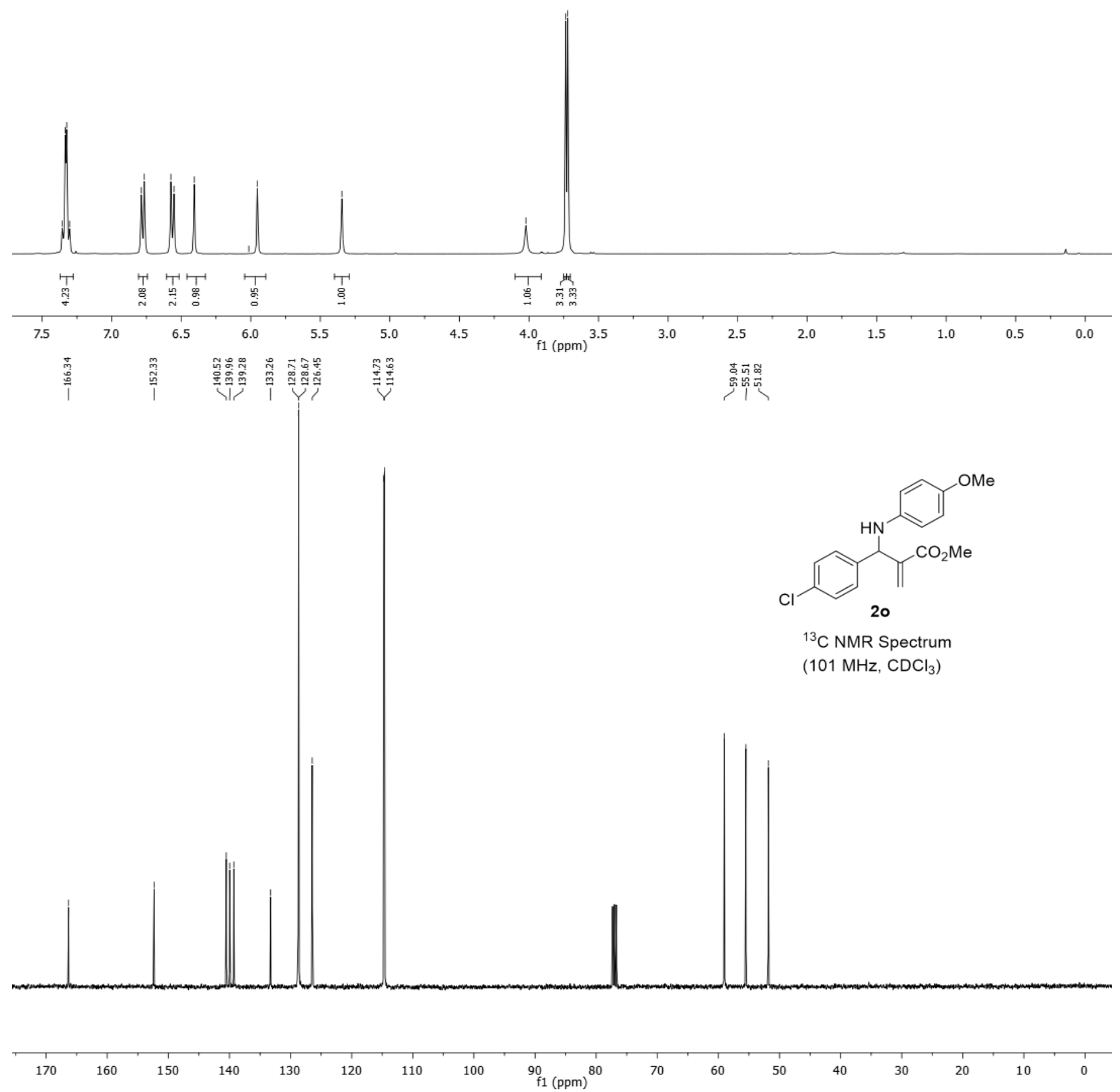

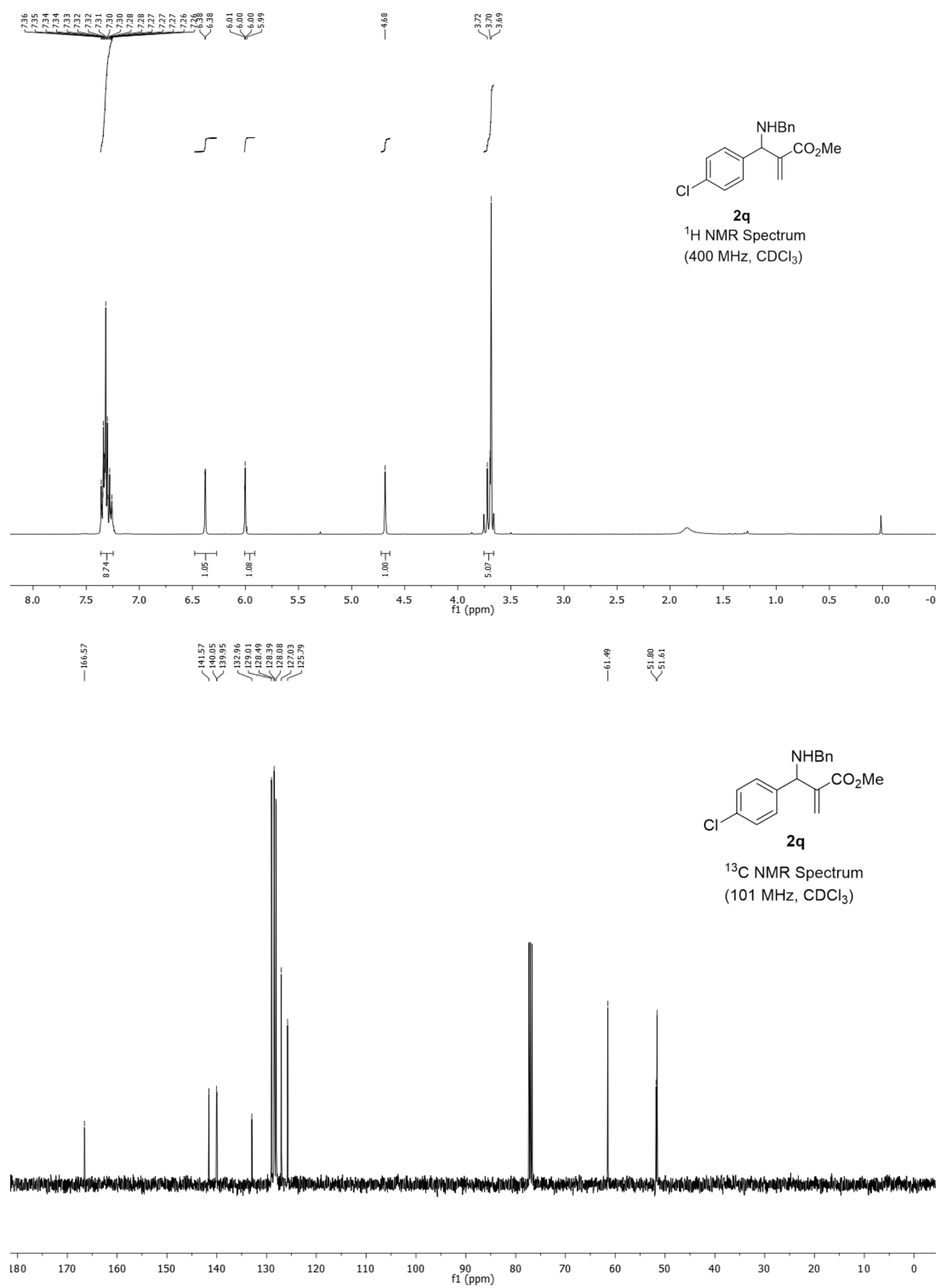

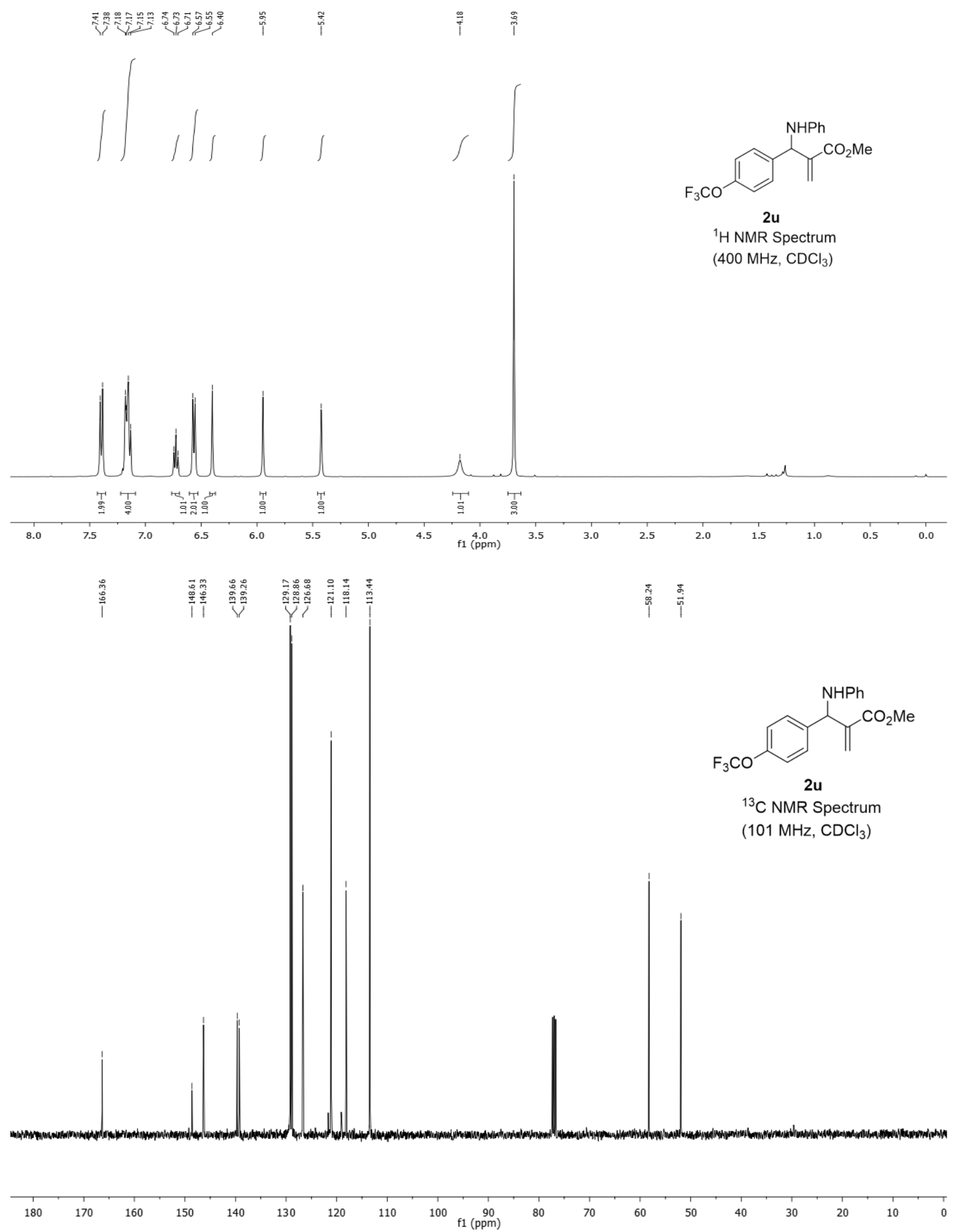


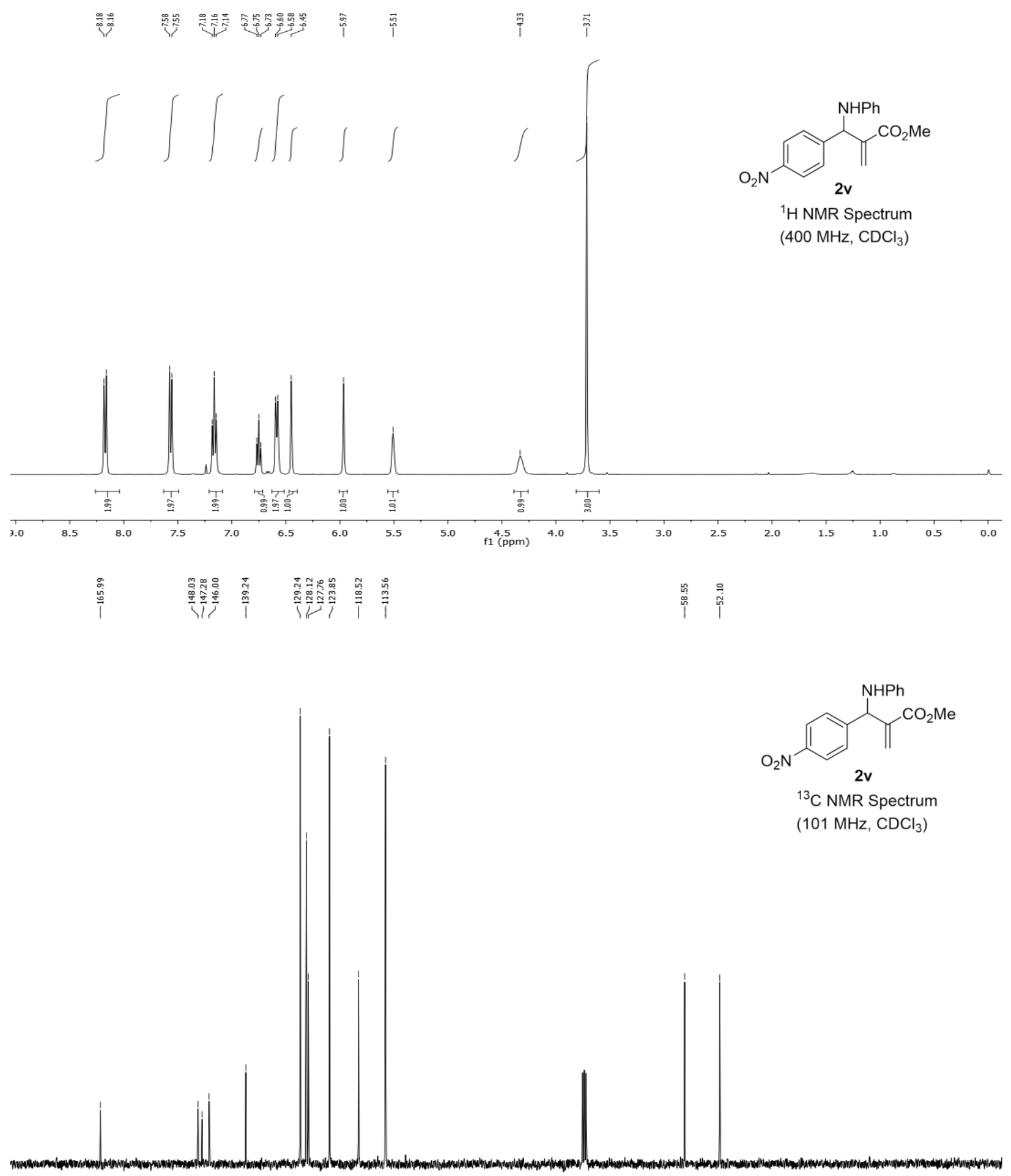

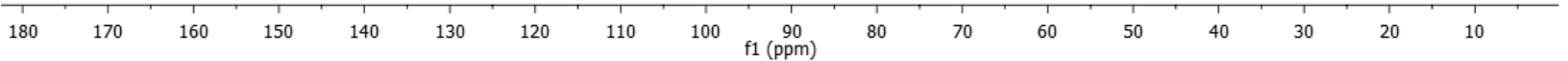



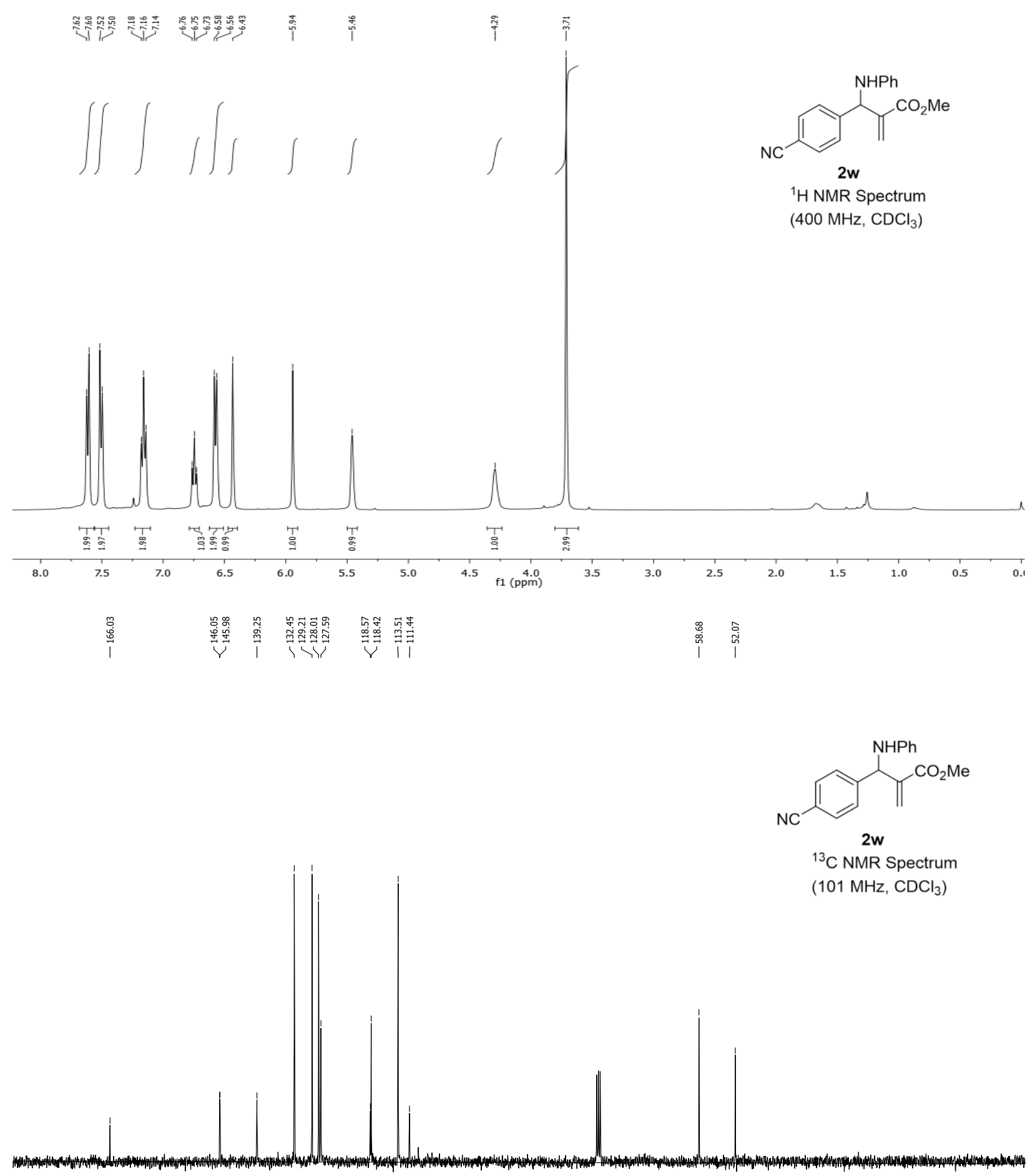

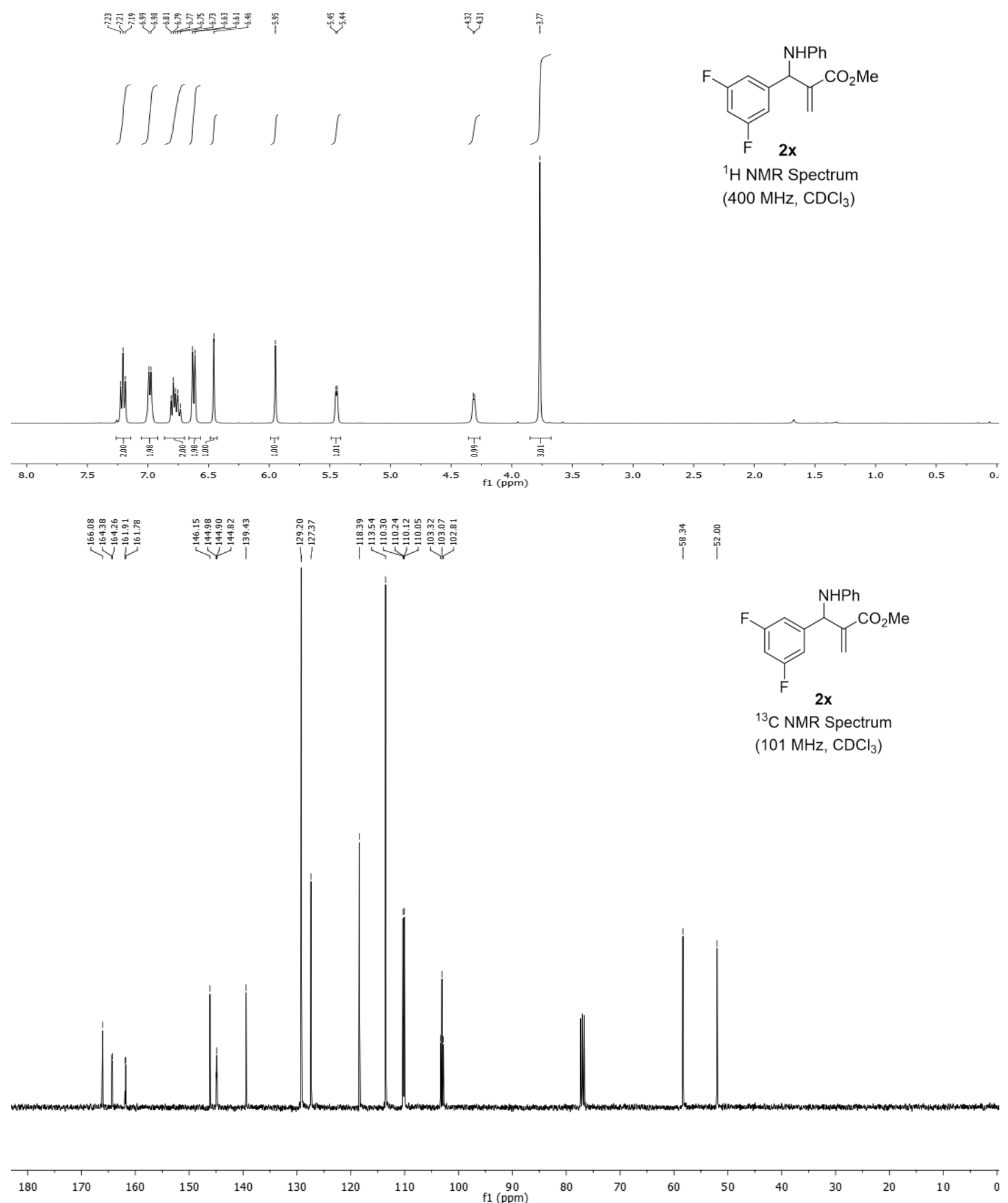

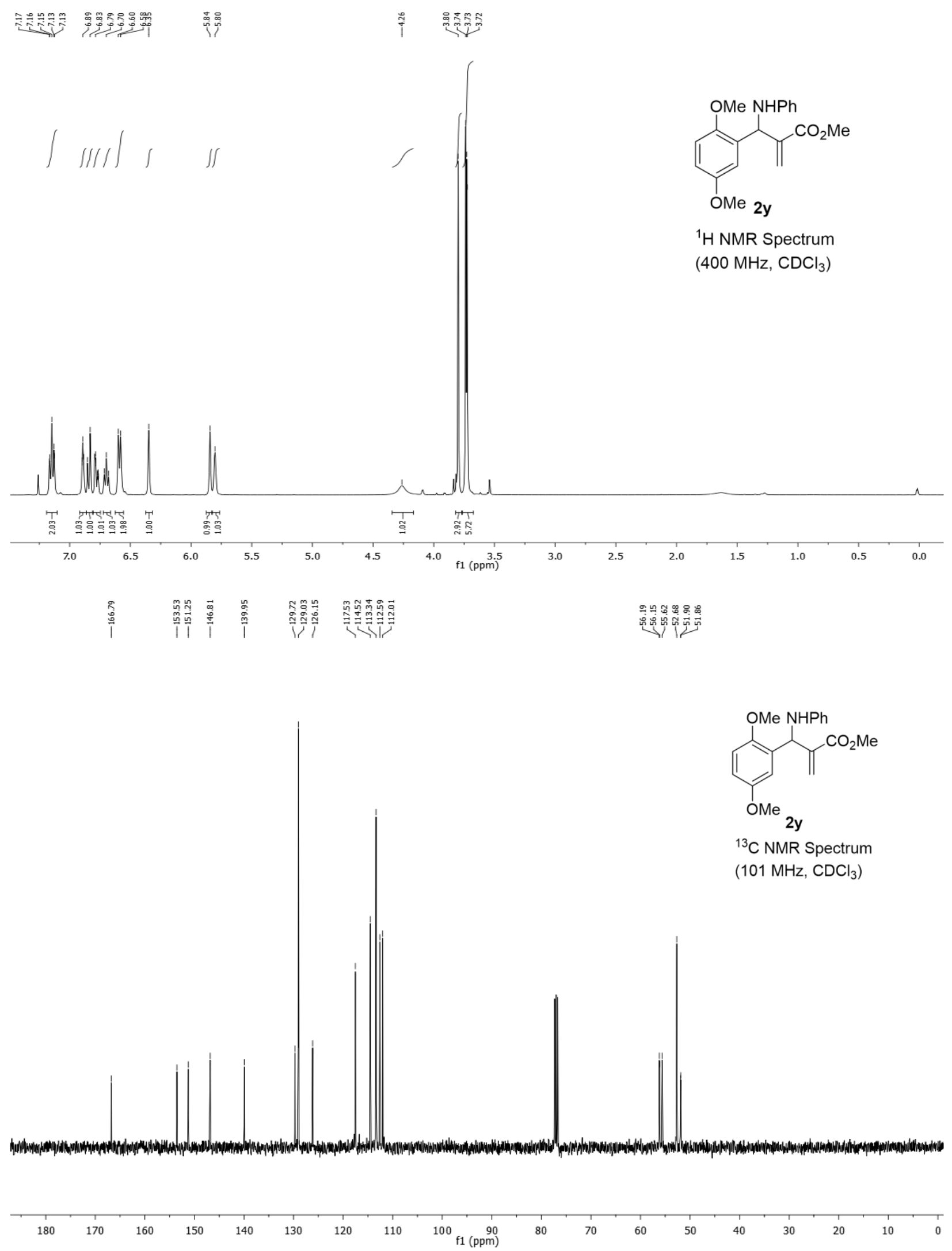

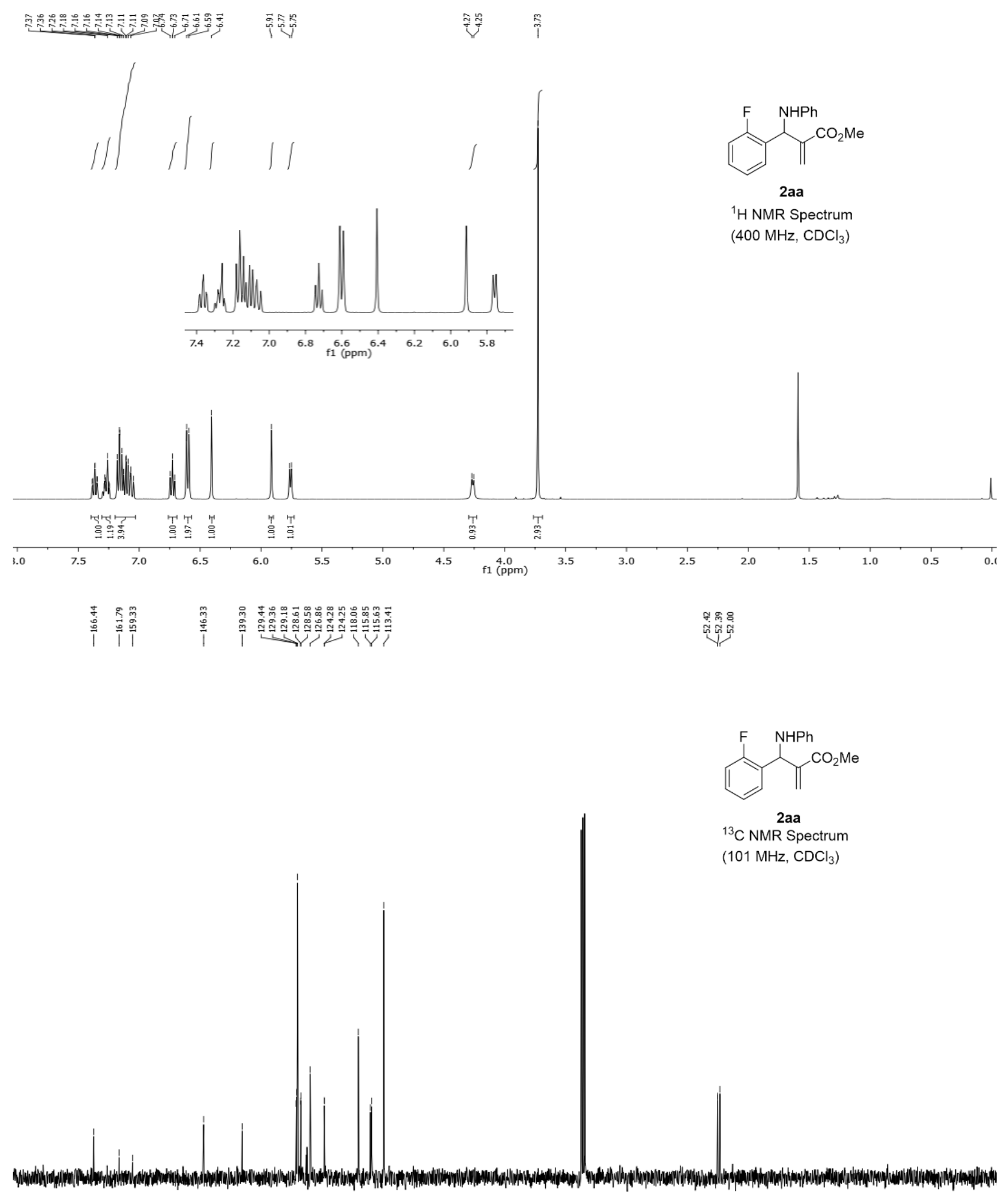

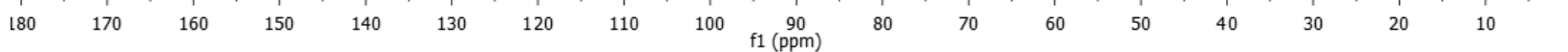




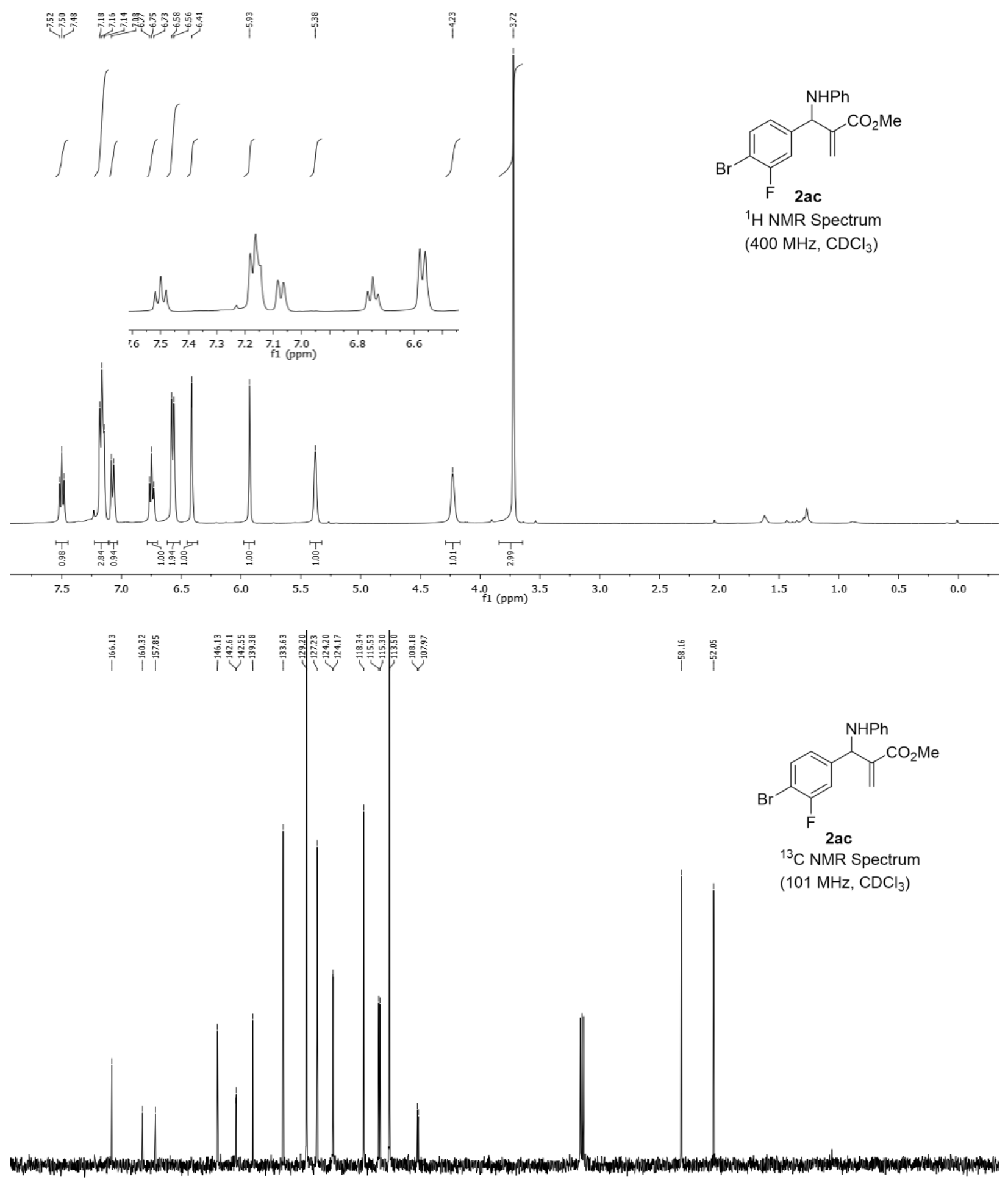

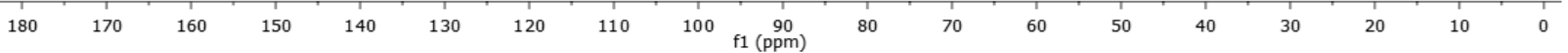




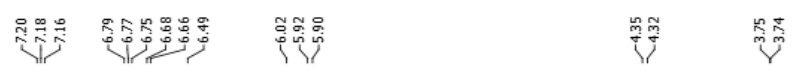
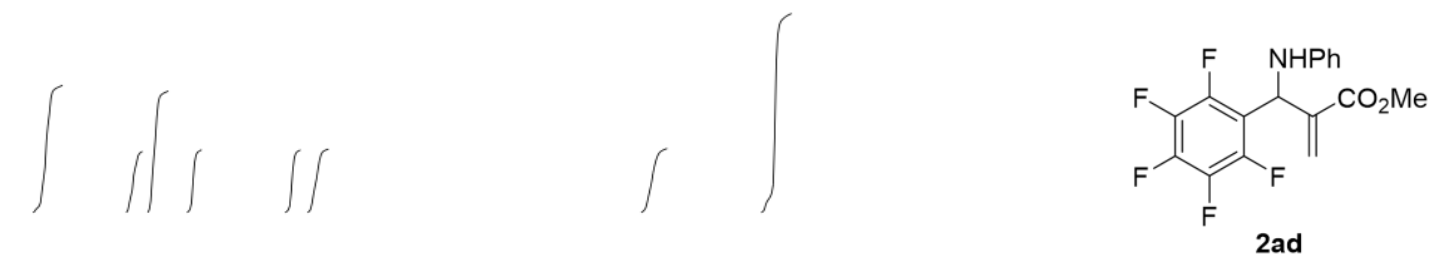

${ }^{1} \mathrm{H}$ NMR Spectrum (400 MHz, $\mathrm{CDCl}_{3}$ )
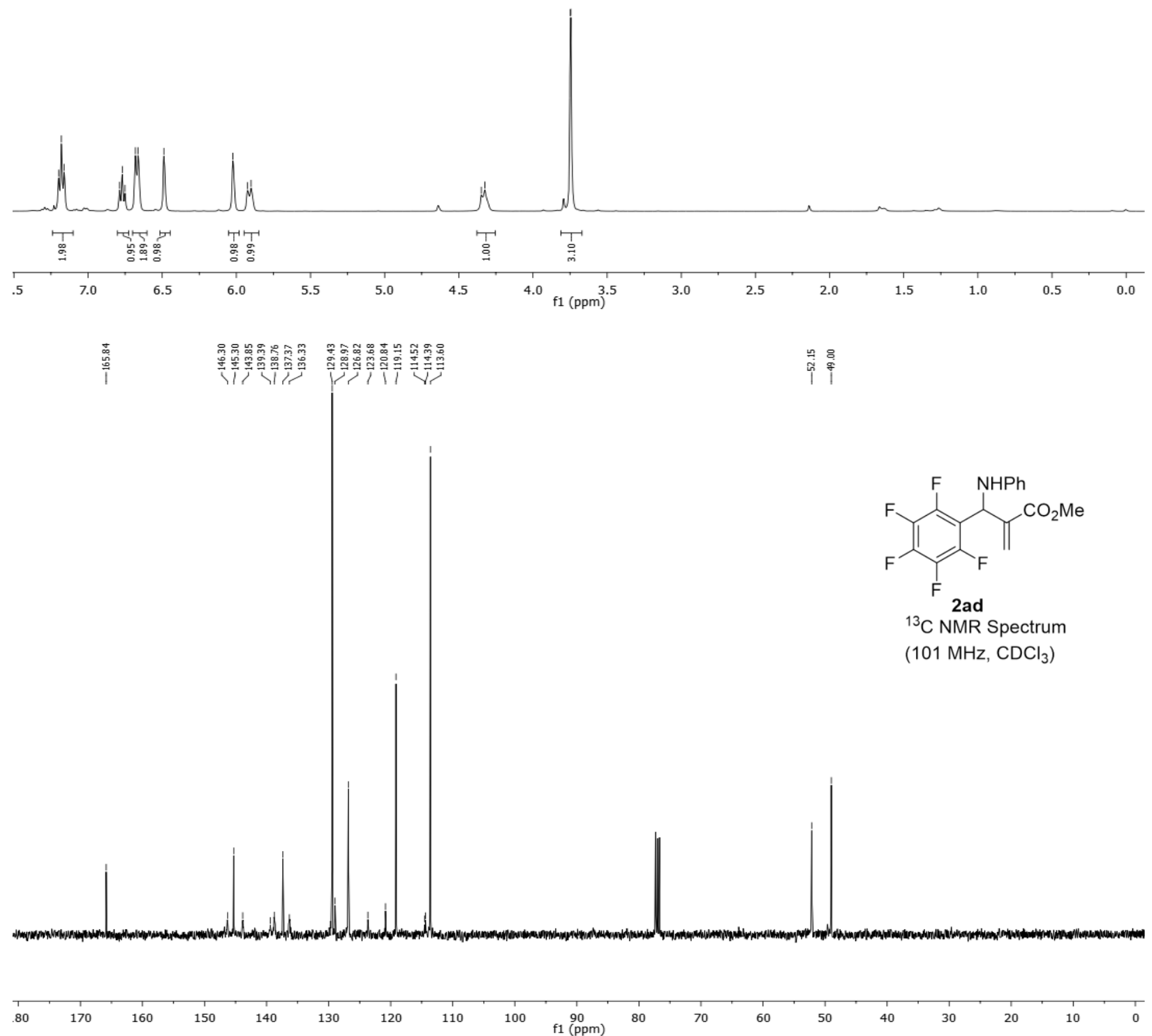

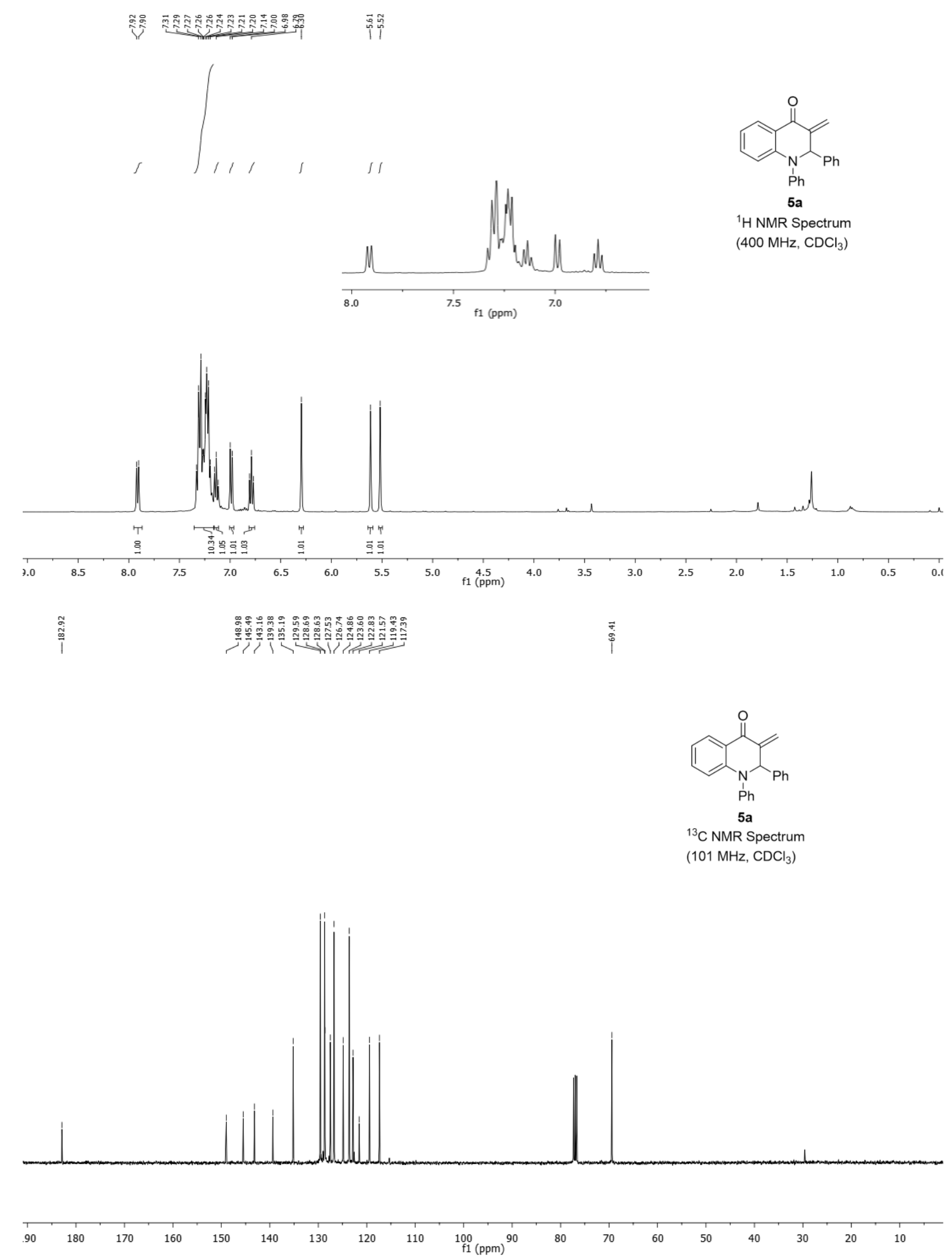


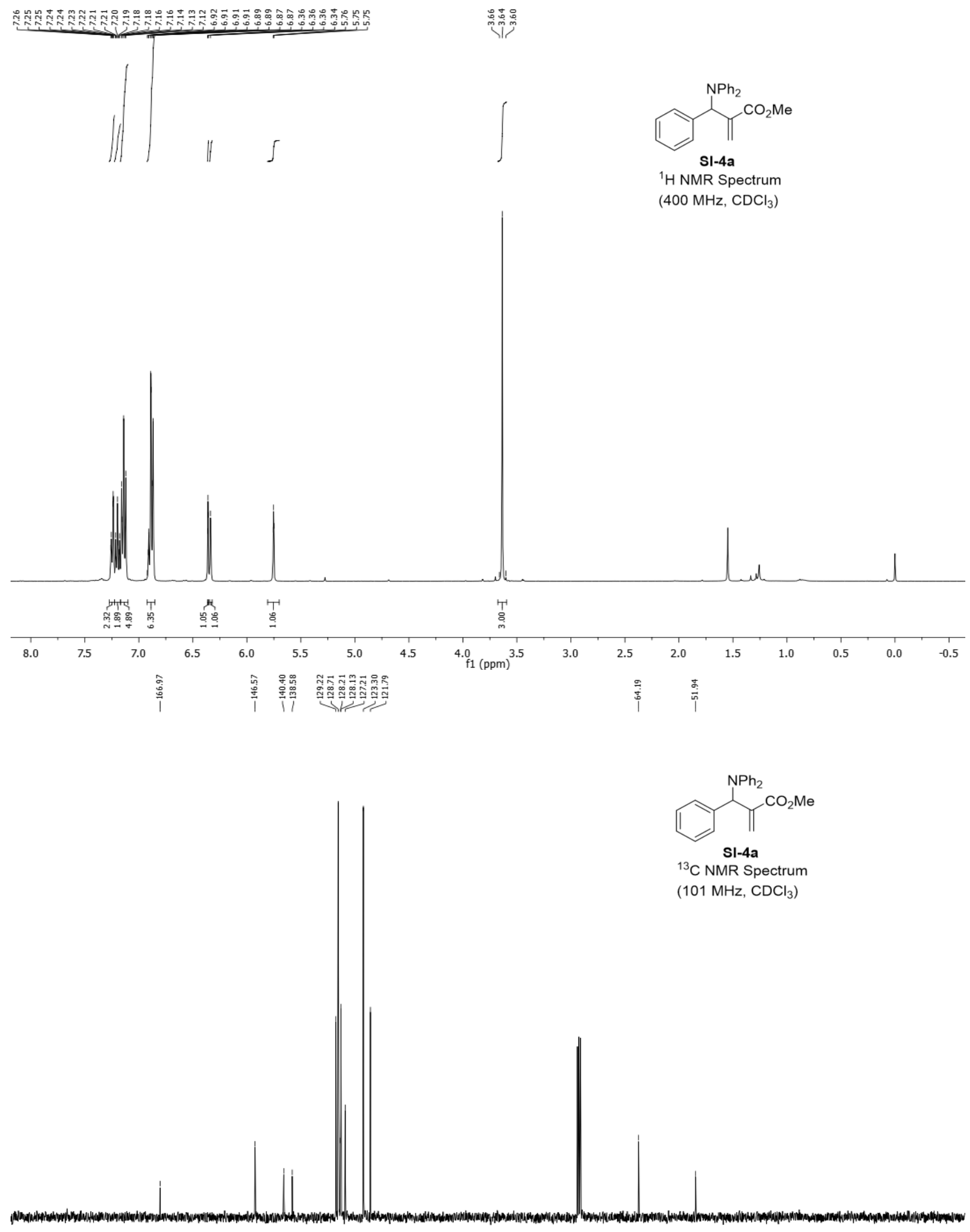

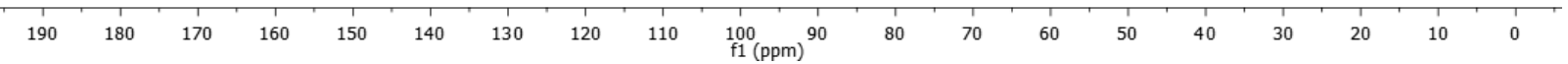




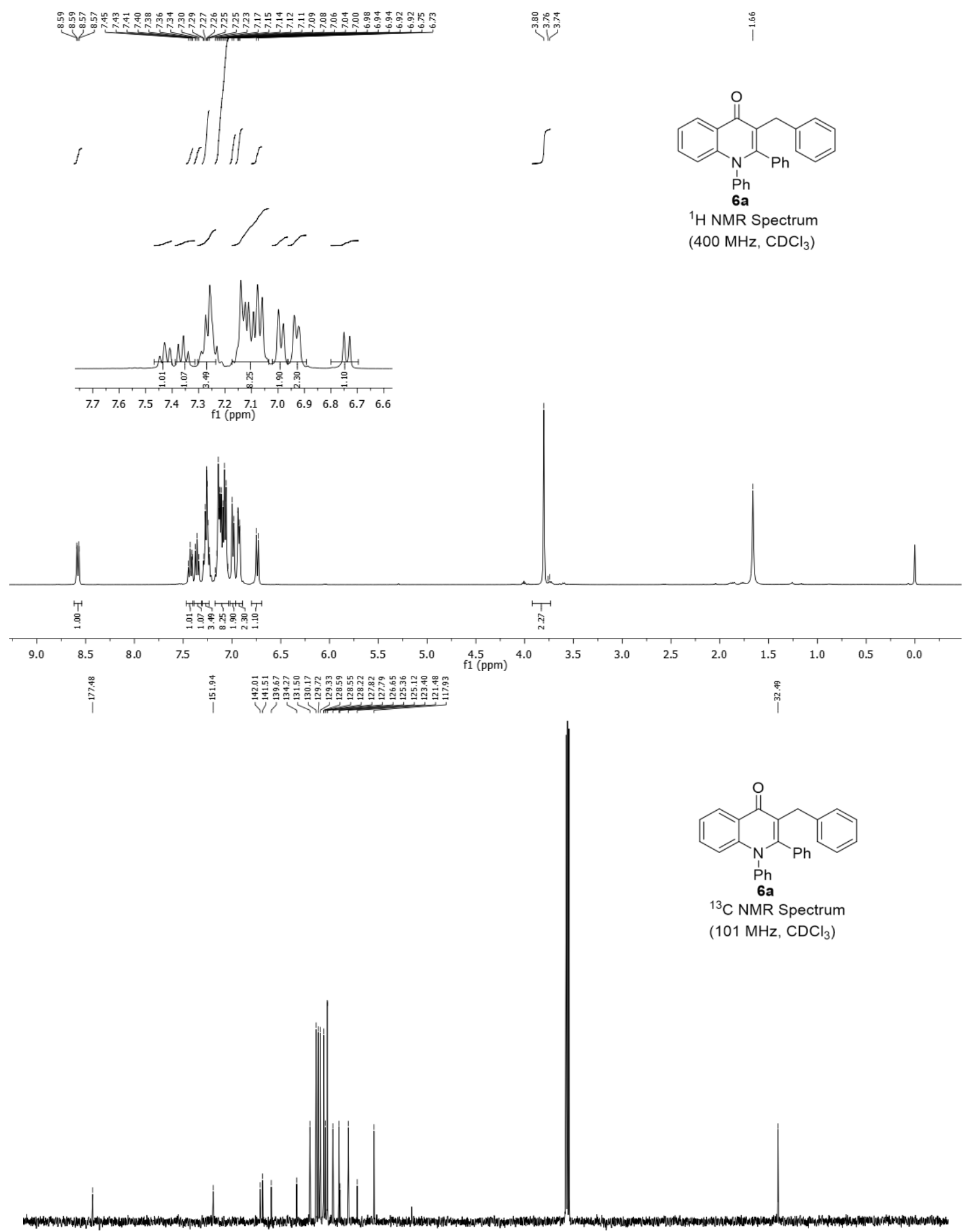

\begin{tabular}{llllllllllllllll}
\hline & 190 & 180 & 170 & 160 & 150 & 140 & 130 & 120 & 110 & 100 & 1 & 1 & 1 & 1 & 1 \\
$\mathrm{f} 1(\mathrm{ppm})$ & 80 & 70 & 60 & 50 & 40 & 30 & 20 & 10 & 0
\end{tabular} 


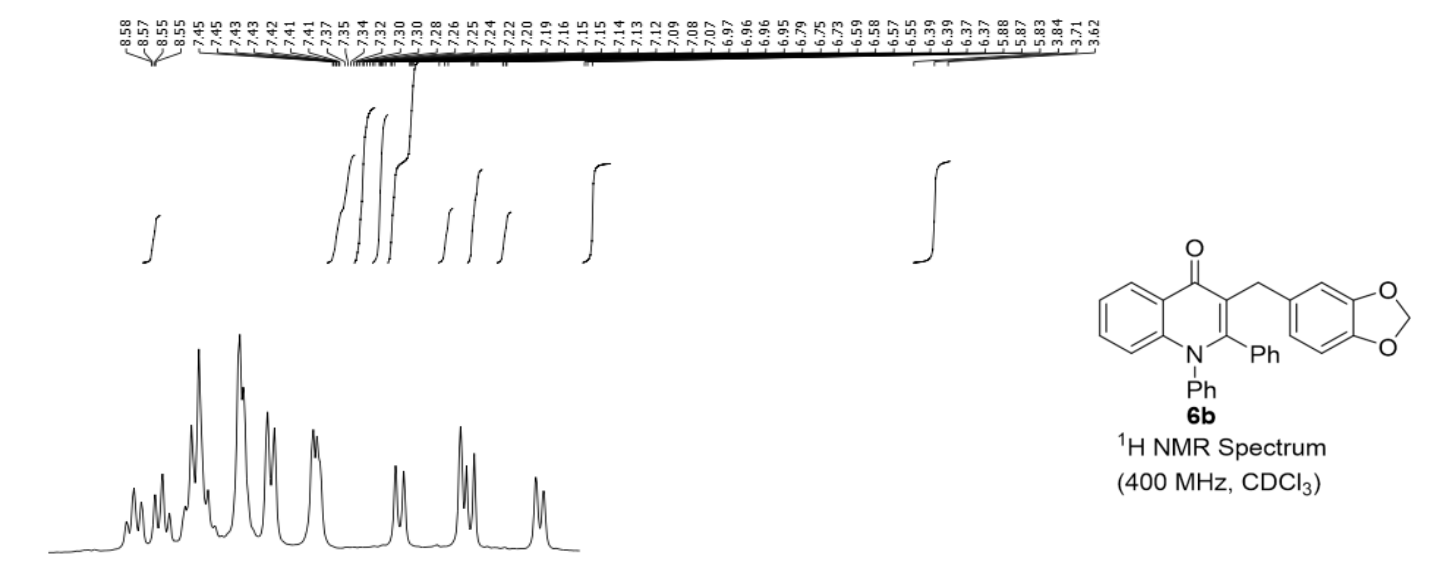

$\begin{array}{lllllllllllllll}7.6 & 7.5 & 7.4 & 7.3 & 7.2 & 7.1 & 7.0 & 6.9 & 6.8 & 6.7 & 6.6 & 6.5 & 6.4 & 6.3\end{array}$
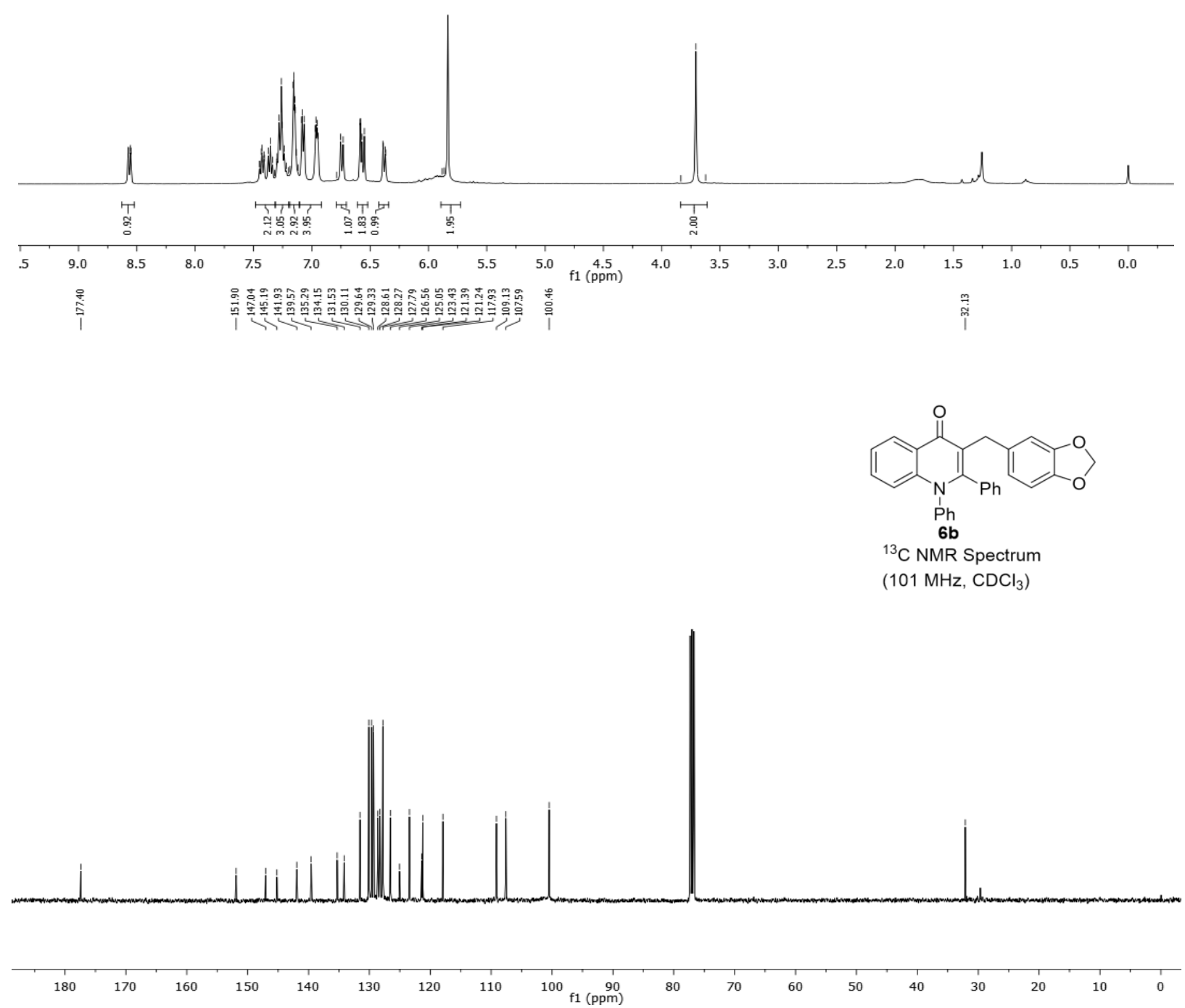

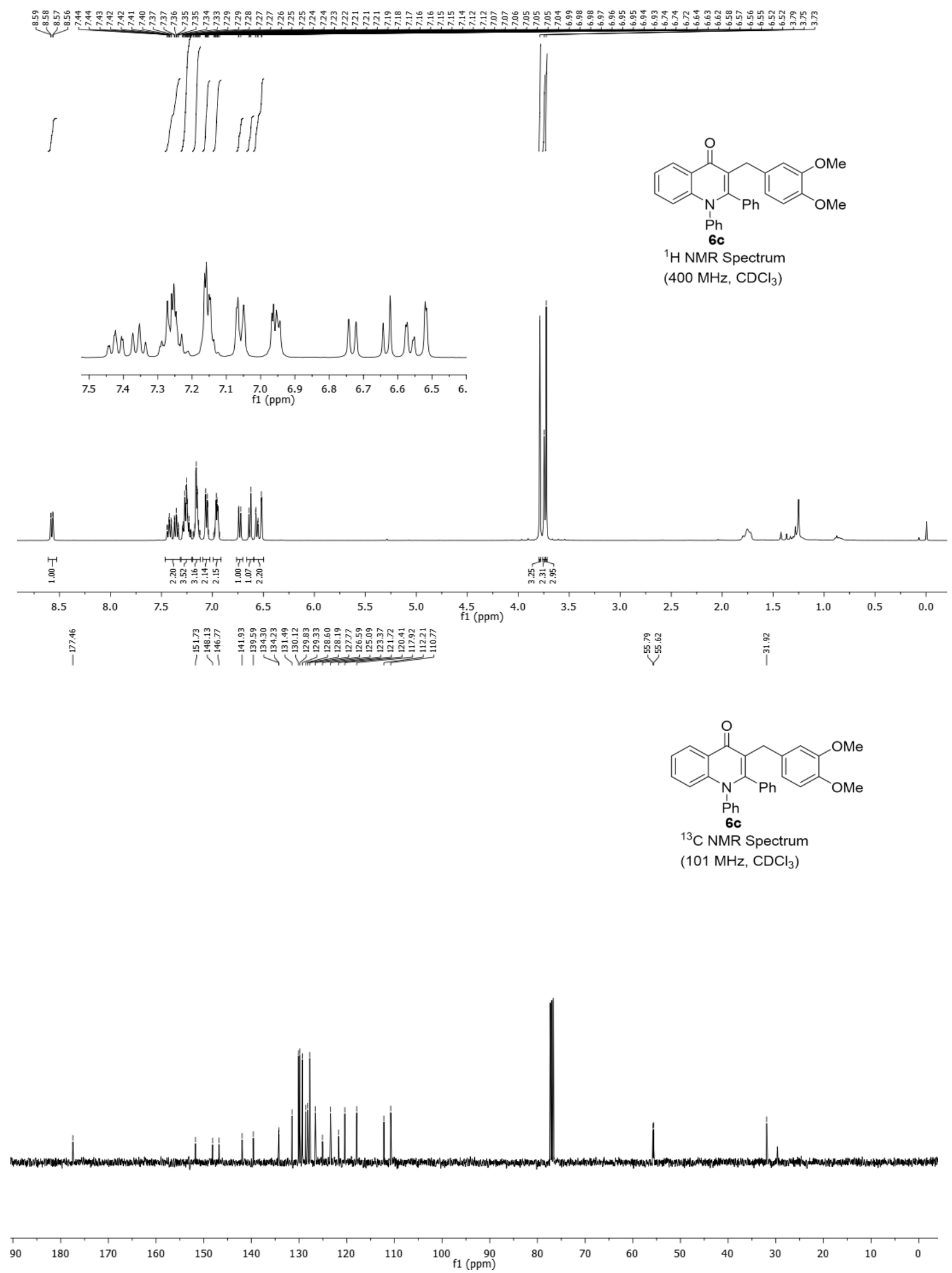

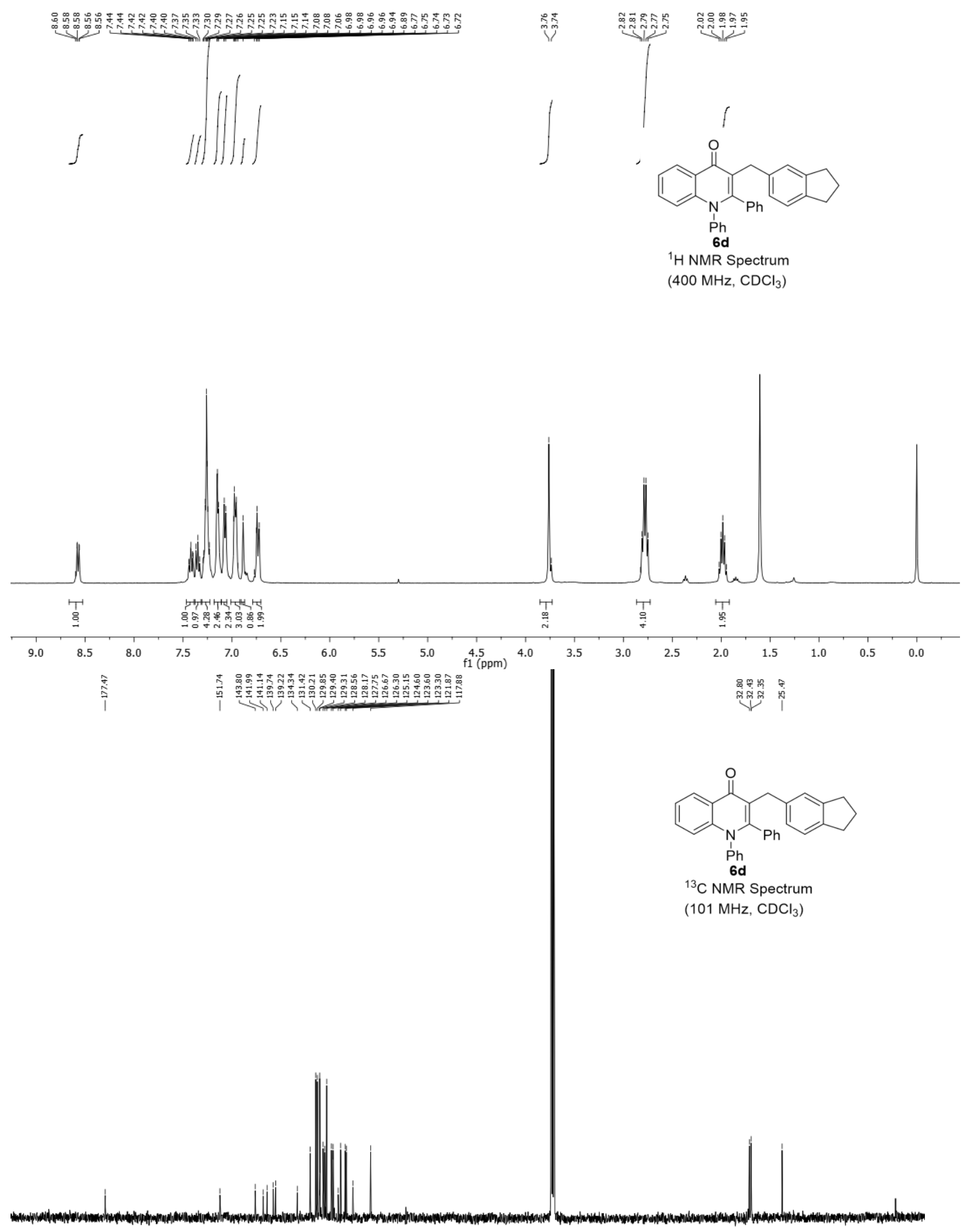

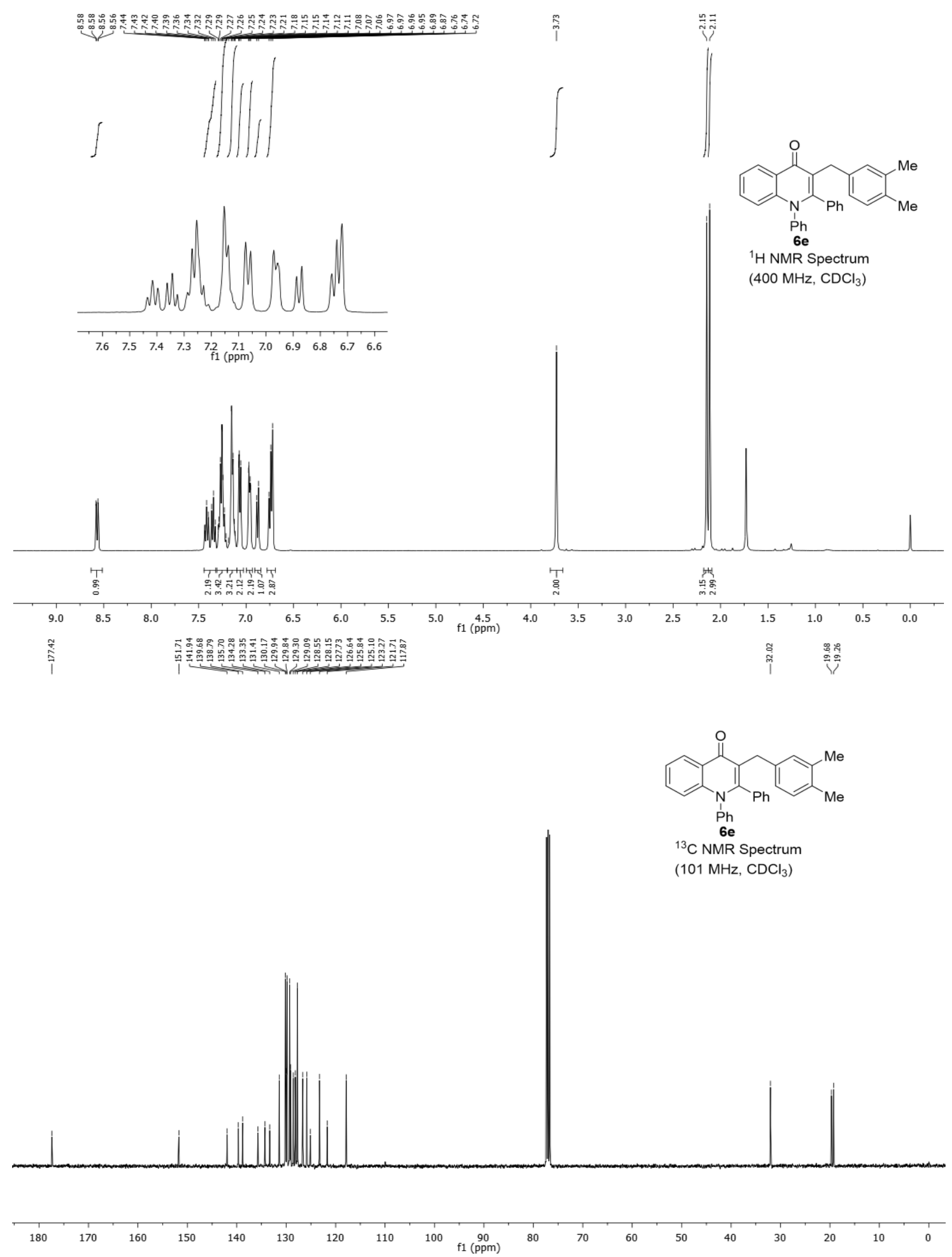


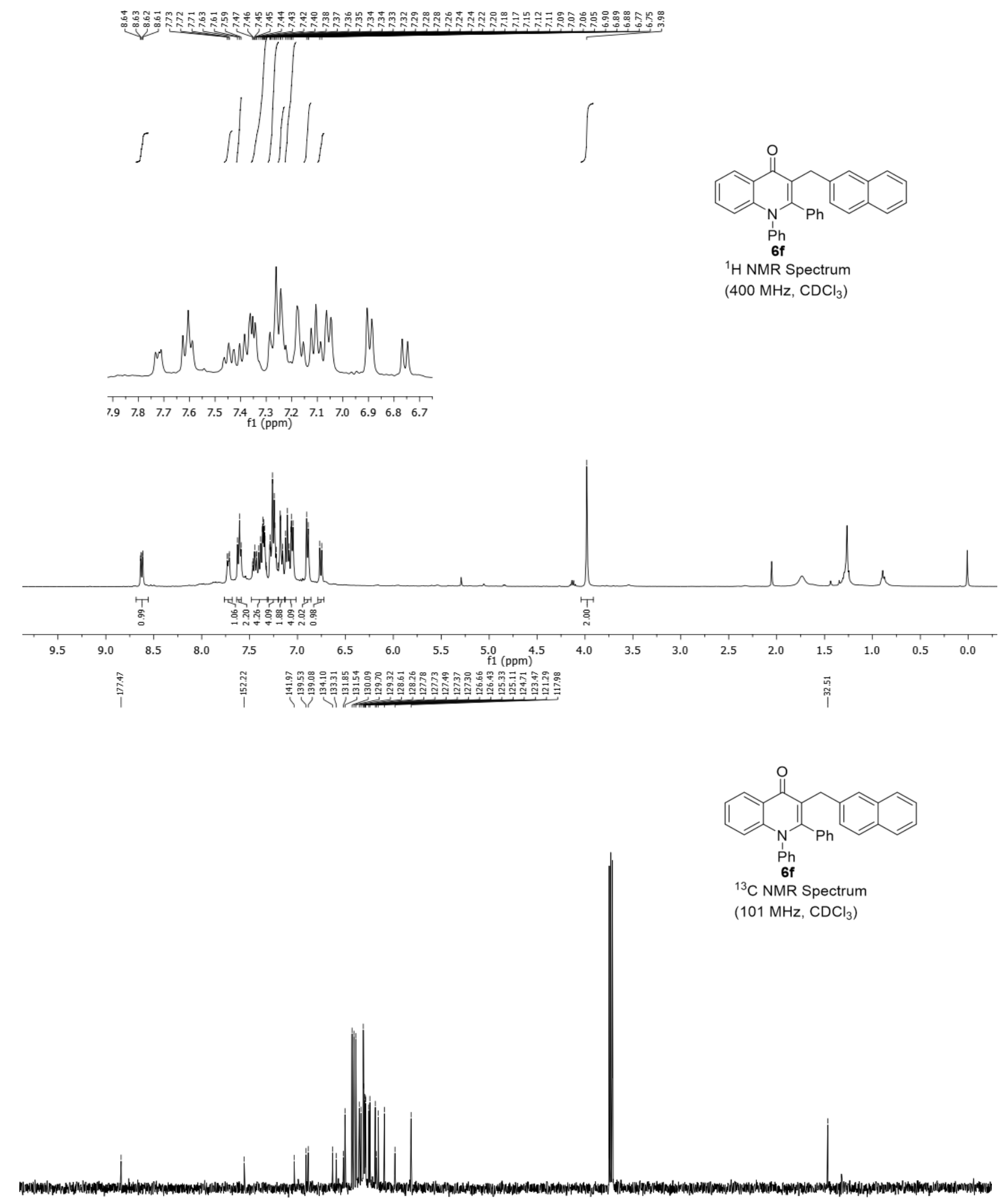



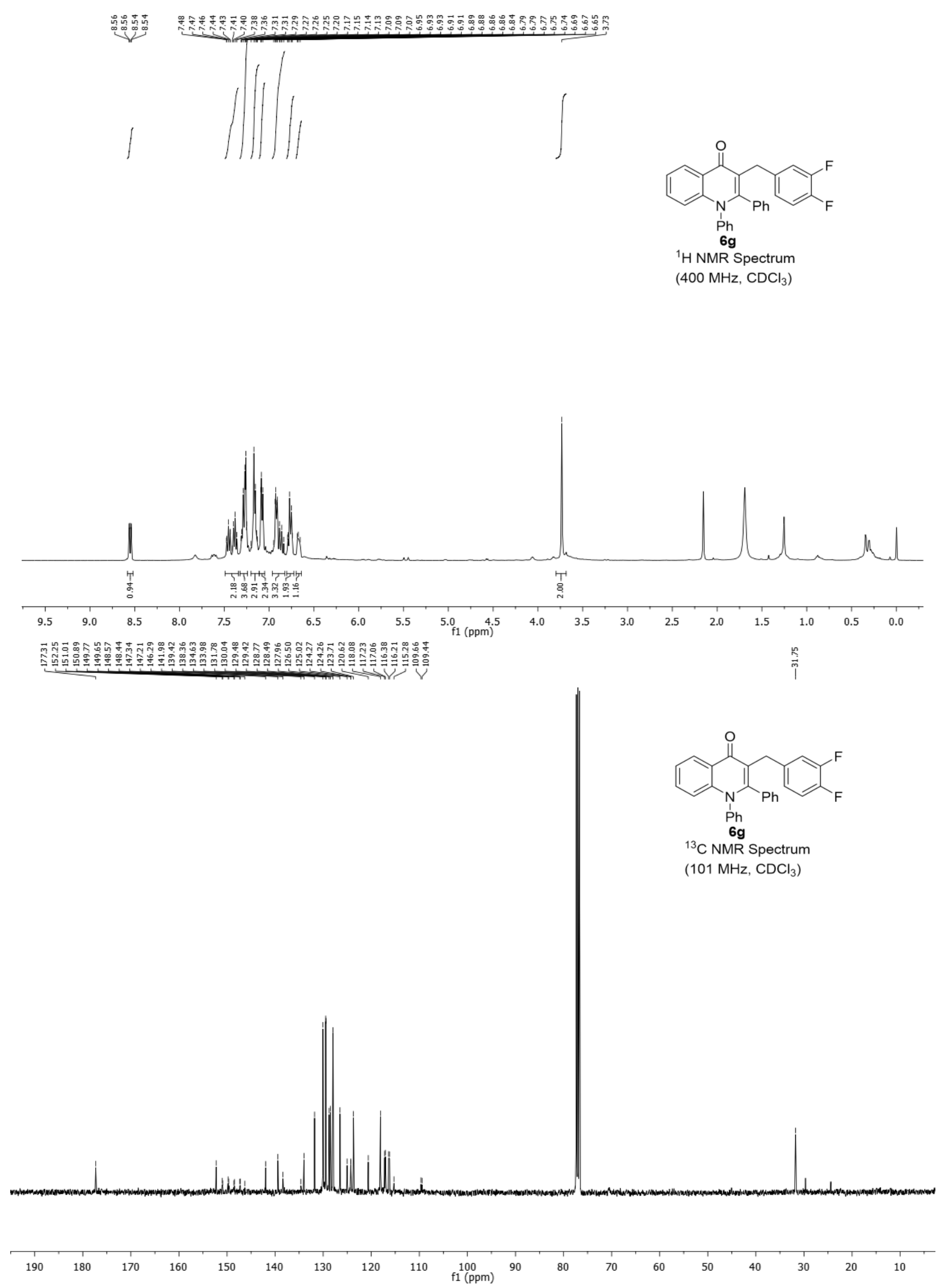

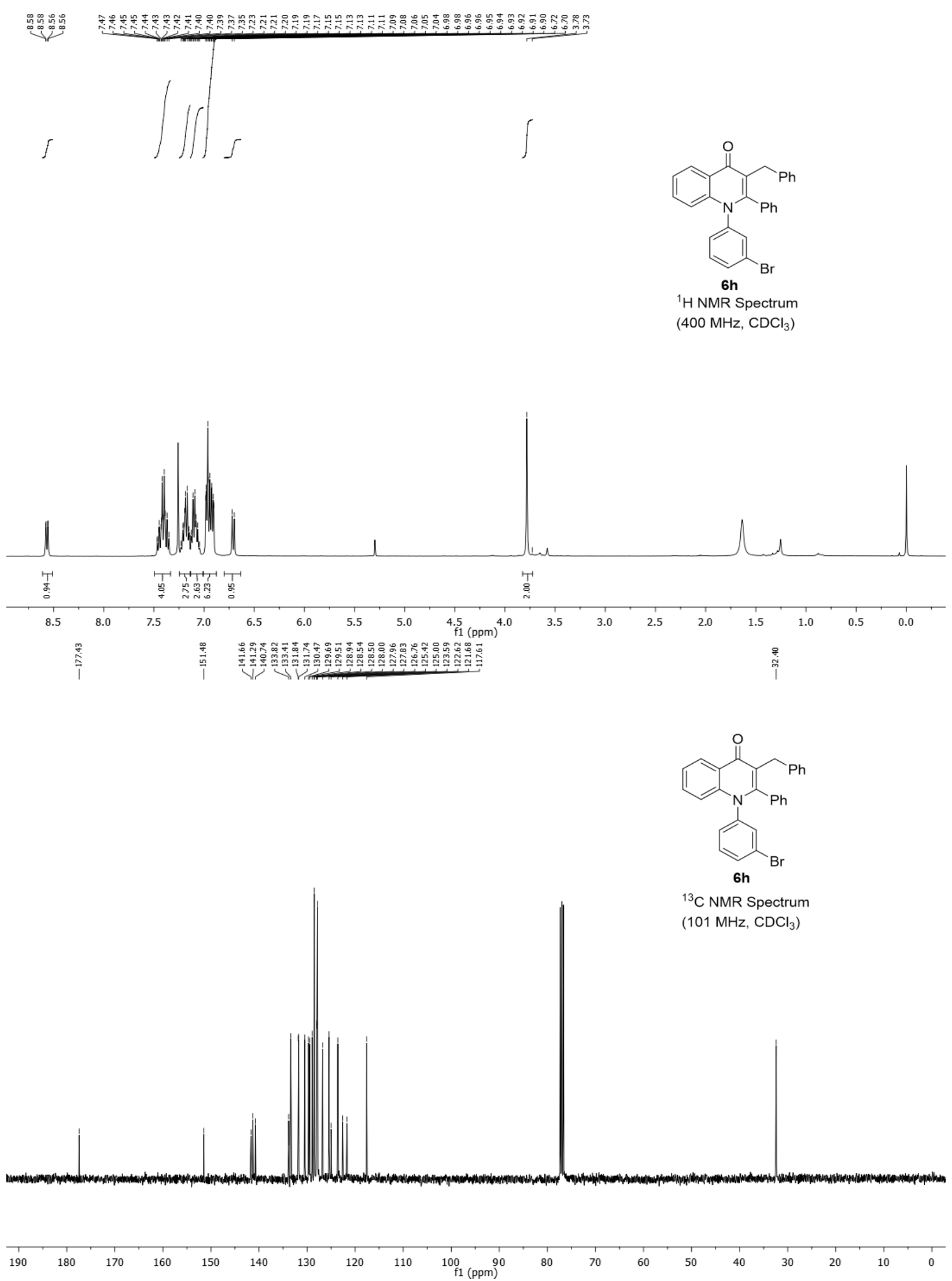

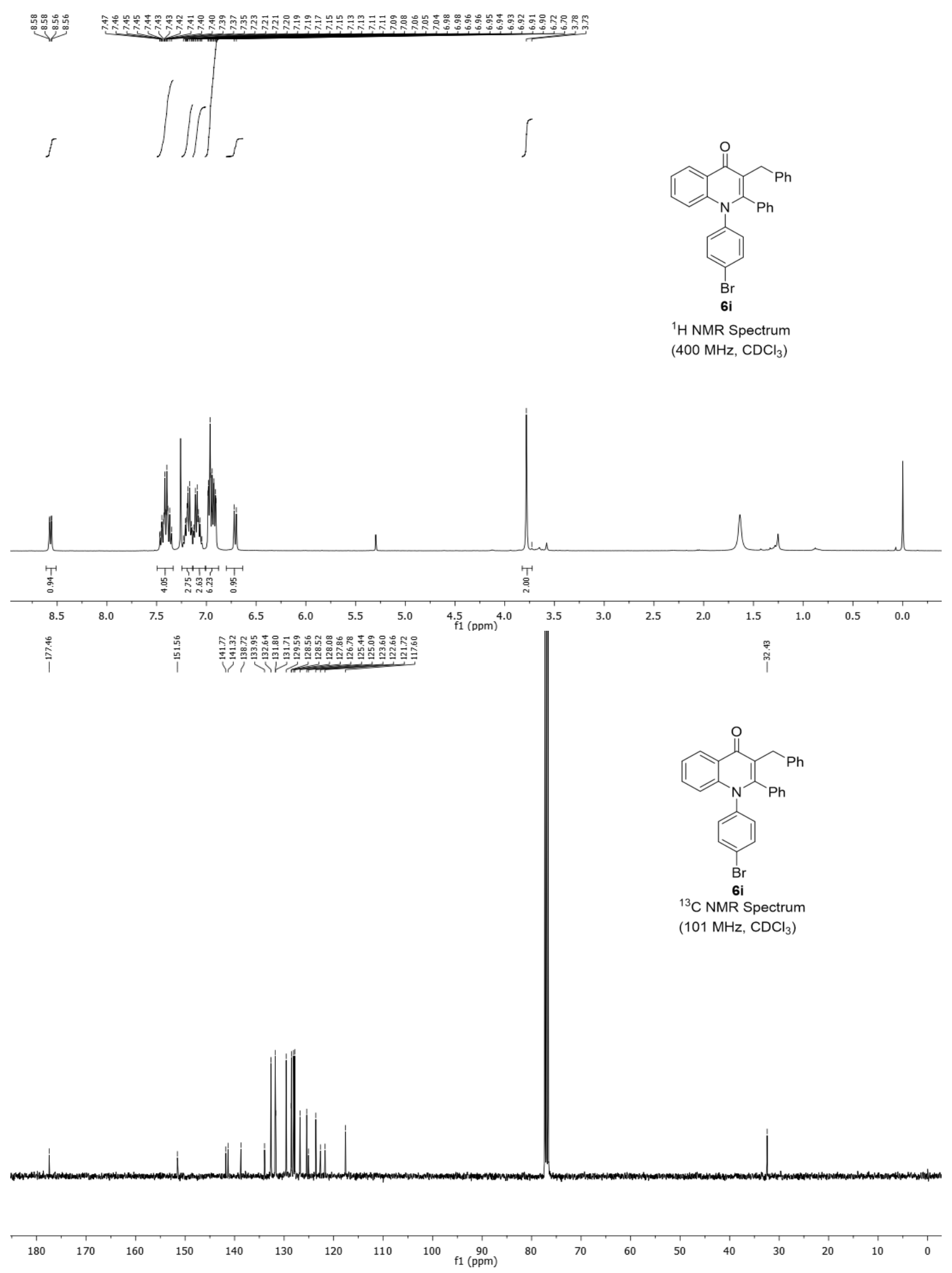

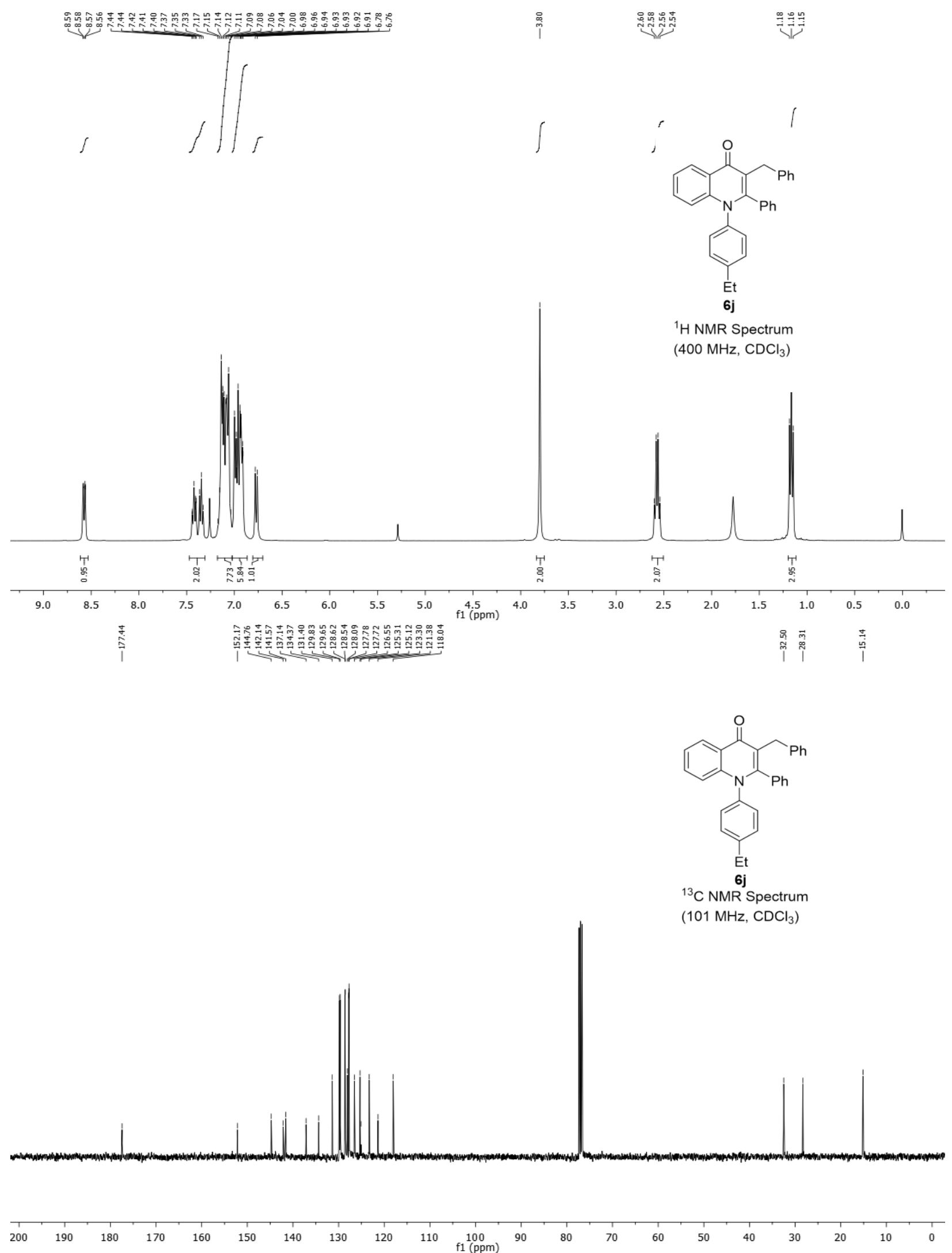

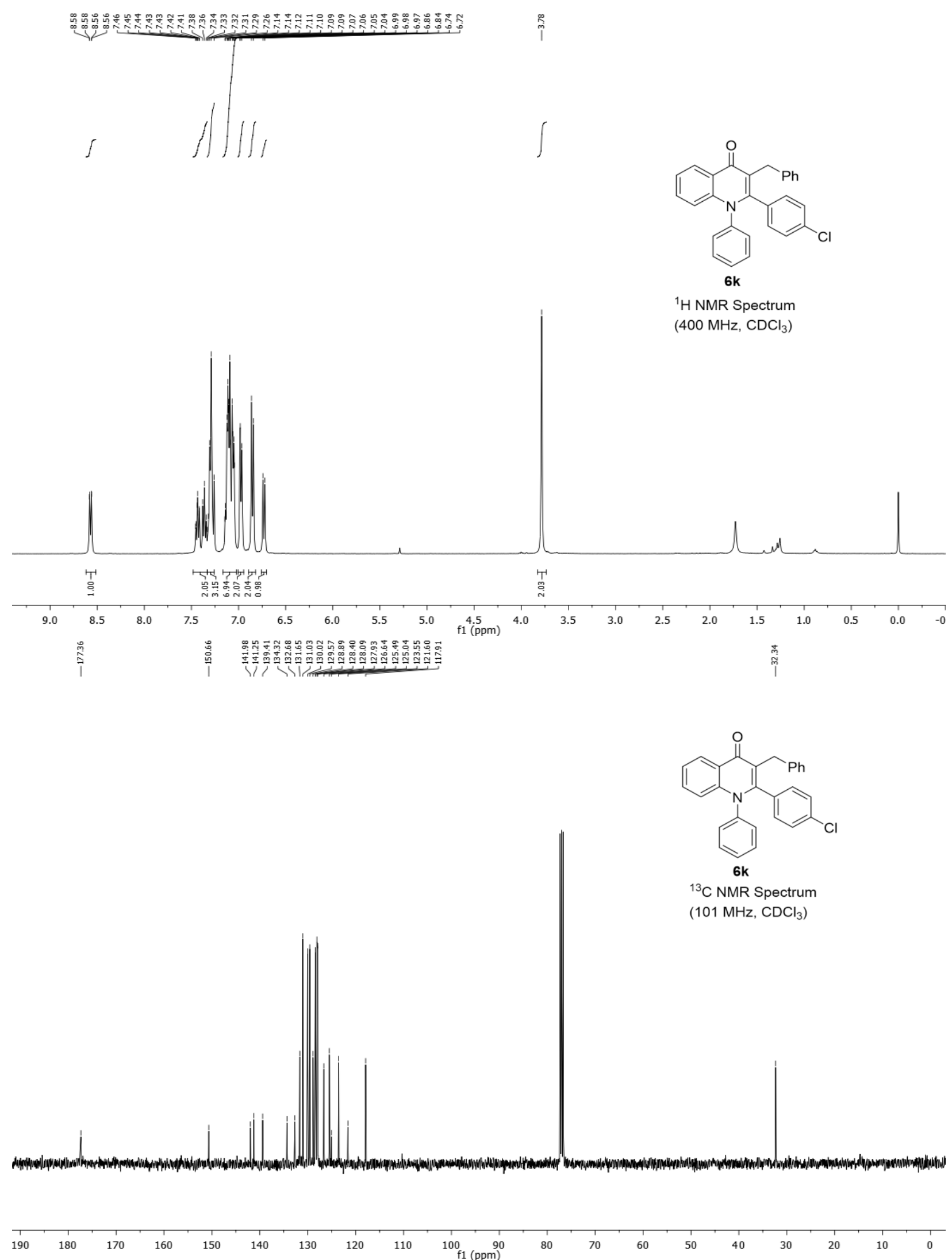


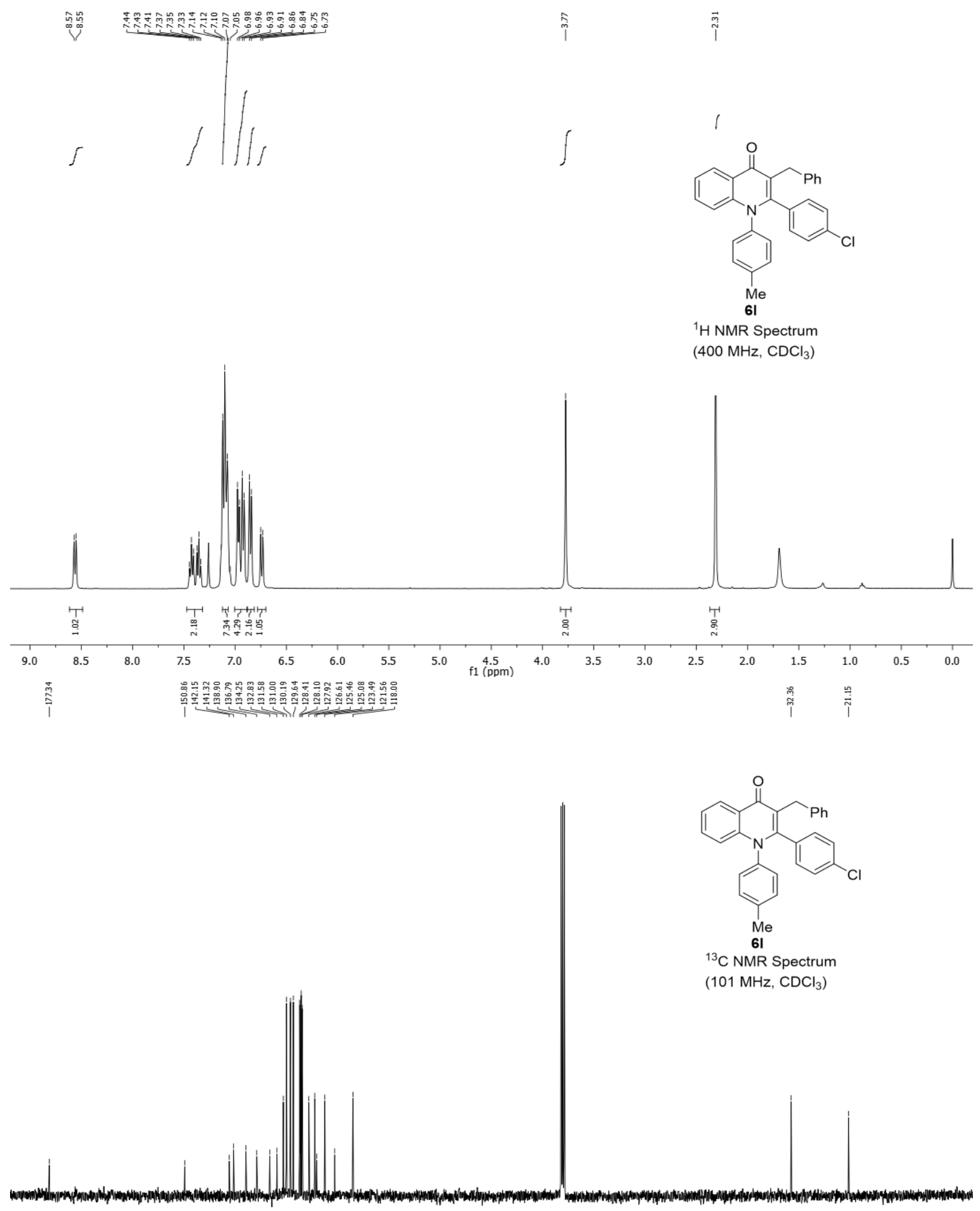

\begin{tabular}{|c|c|c|c|c|c|c|c|c|c|c|c|c|c|c|c|c|c|c|}
\hline 180 & 170 & 160 & 150 & 140 & 130 & 120 & 110 & 100 & $\mathrm{f} 1\left(\begin{array}{c}90 \\
\mathrm{ppm}\end{array}\right)$ & 80 & 70 & 60 & 50 & 40 & 30 & 20 & 10 & 0 \\
\hline
\end{tabular}




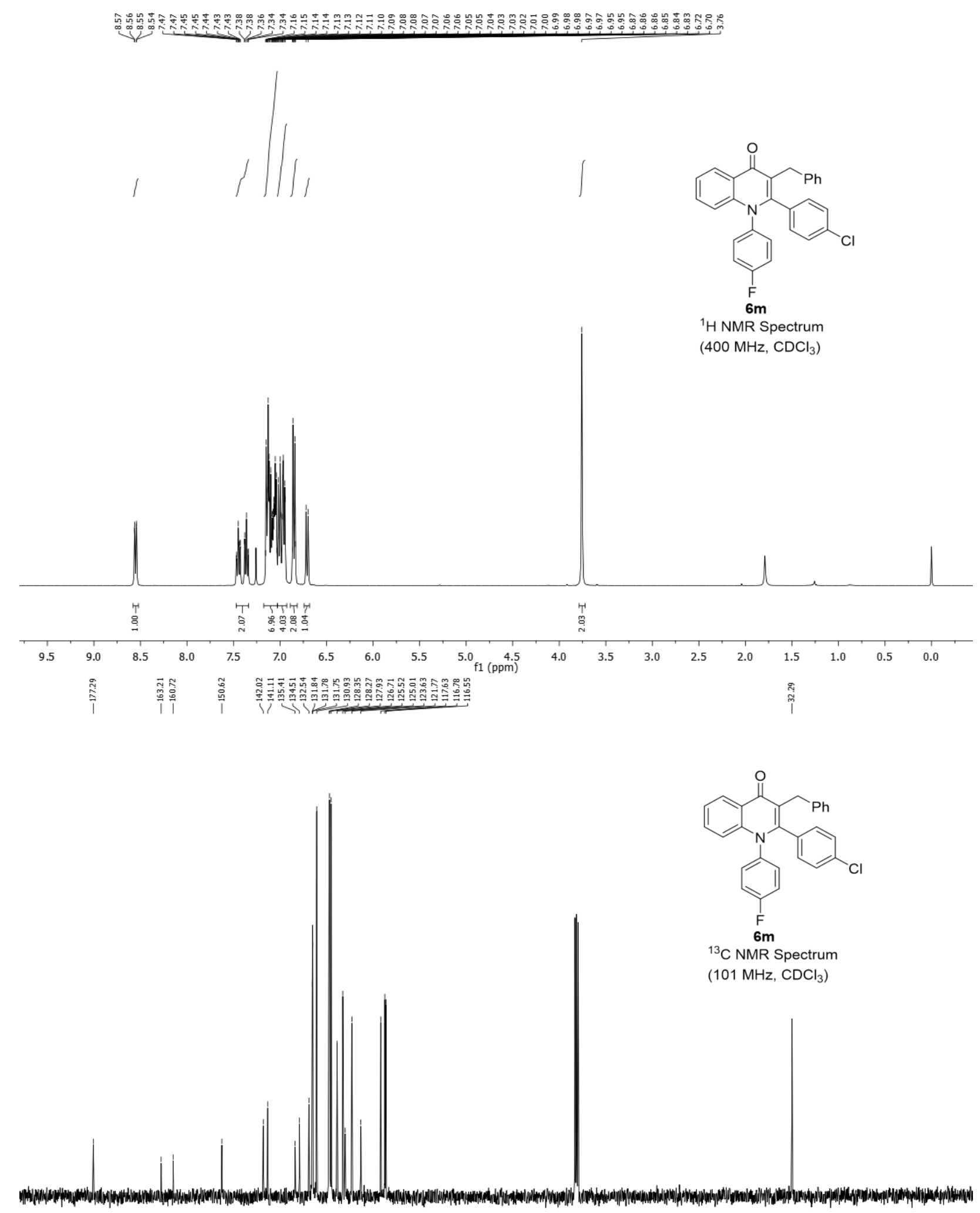

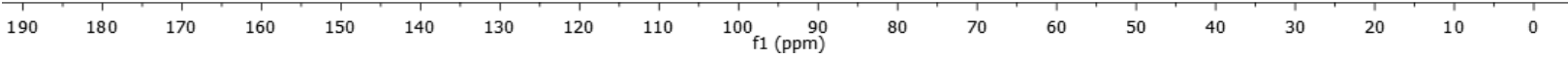



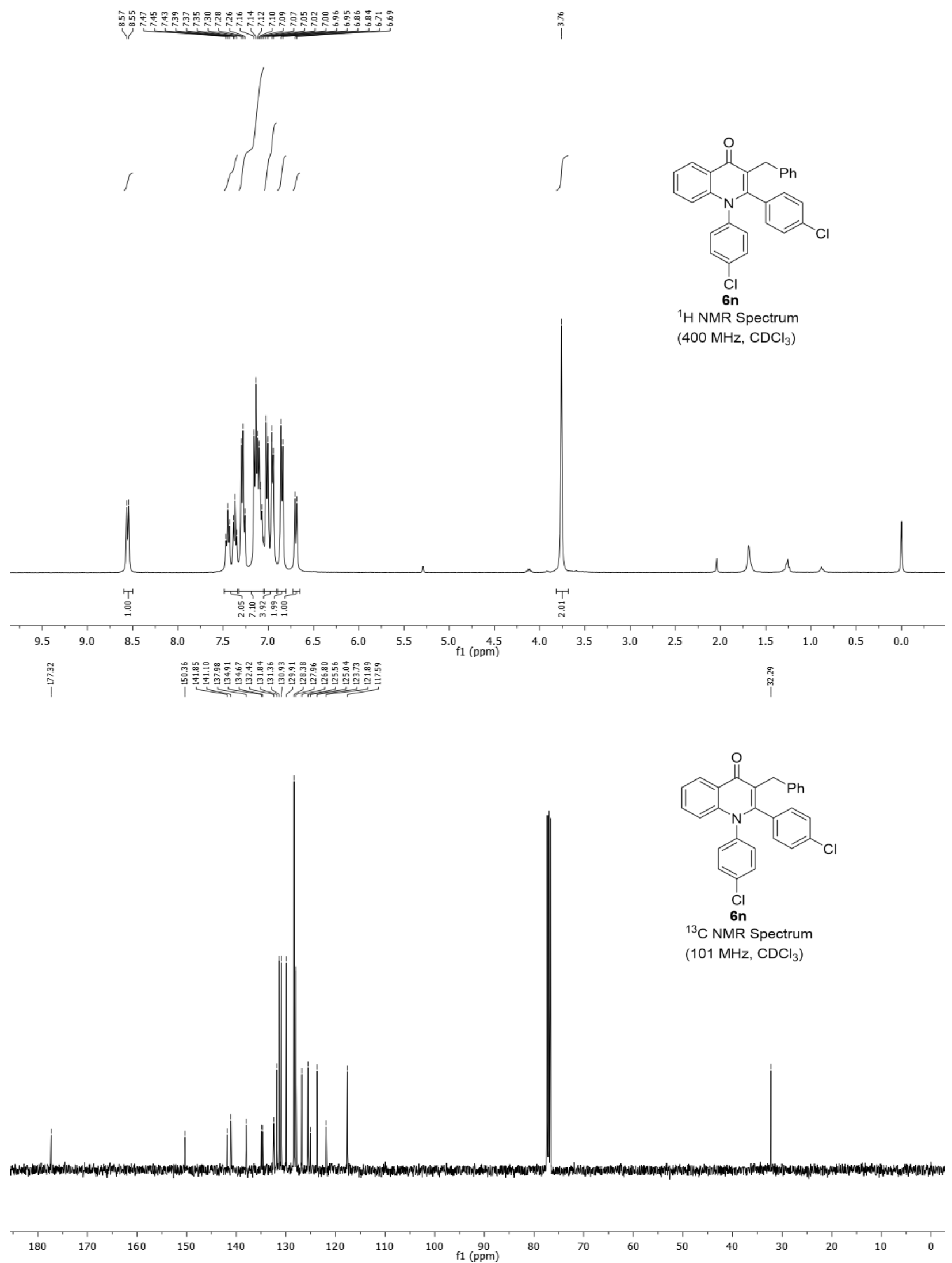


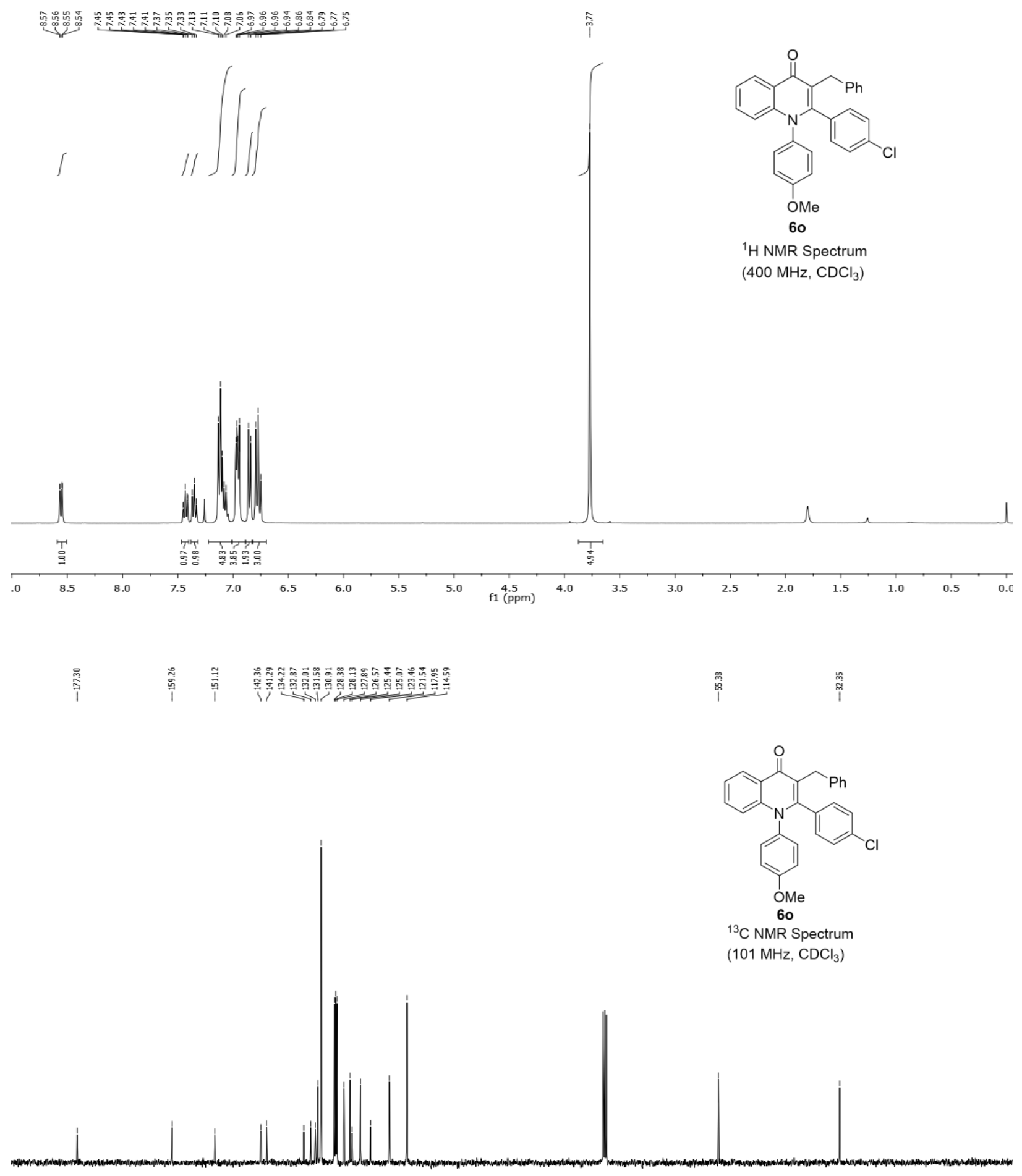

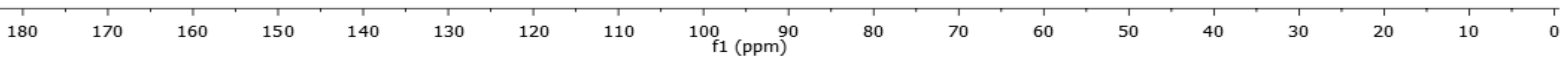



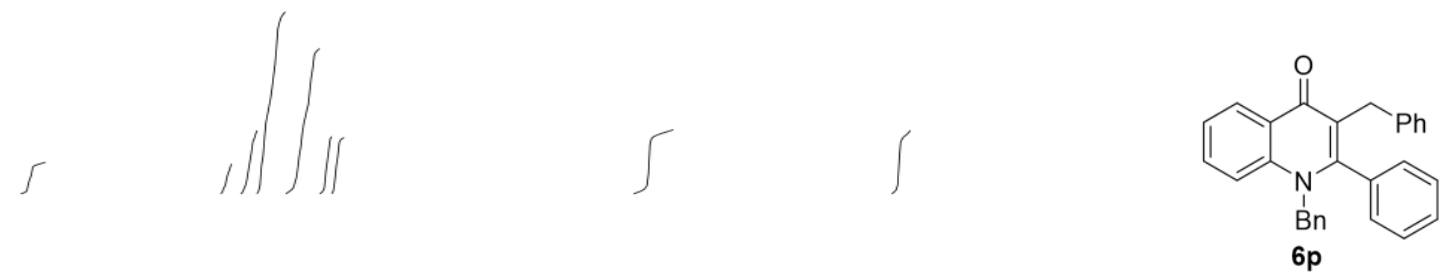

${ }^{1} \mathrm{H}$ NMR Spectrum (400 MHz, $\mathrm{CDCl}_{3}$ )
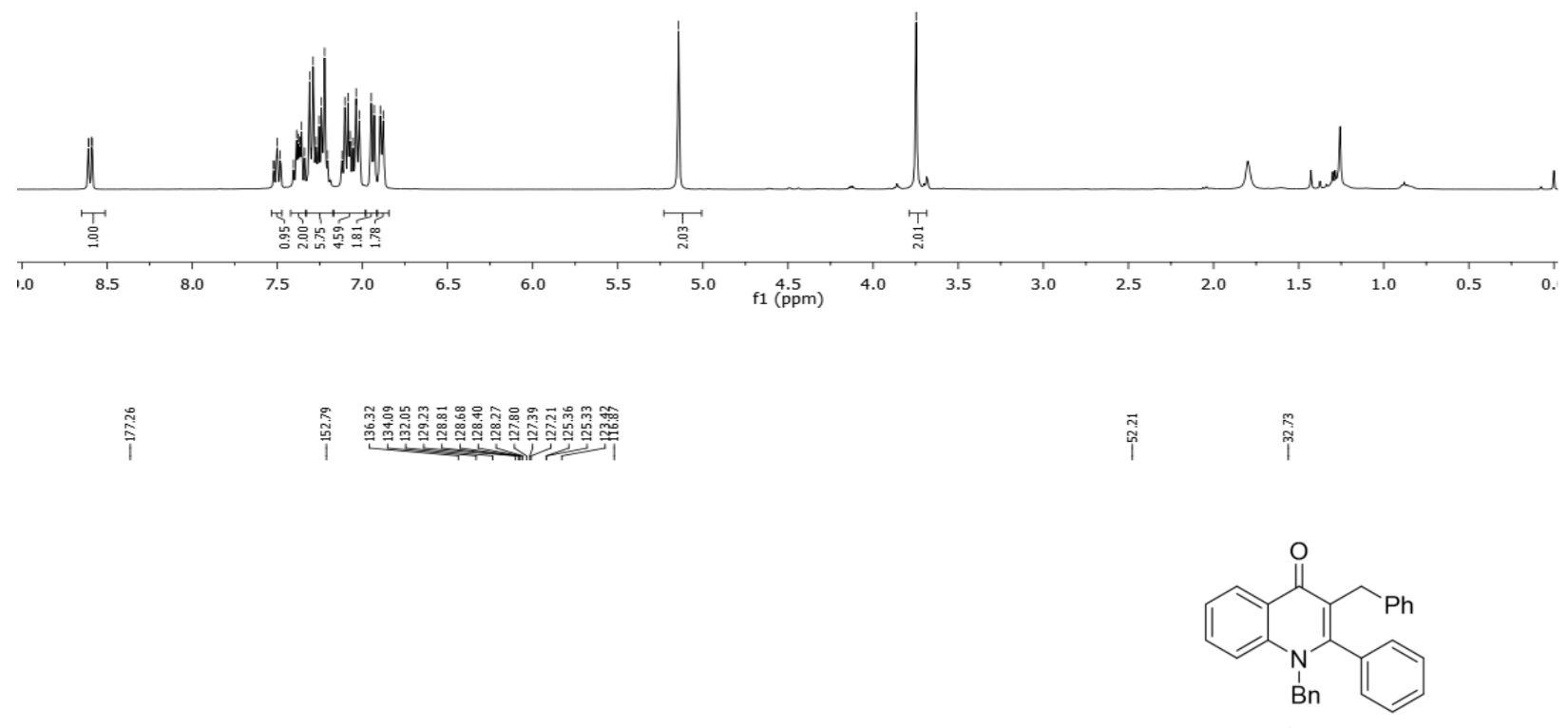

6p

${ }^{13} \mathrm{C}$ NMR Spectrum $\left(101 \mathrm{MHz}, \mathrm{CDCl}_{3}\right)$

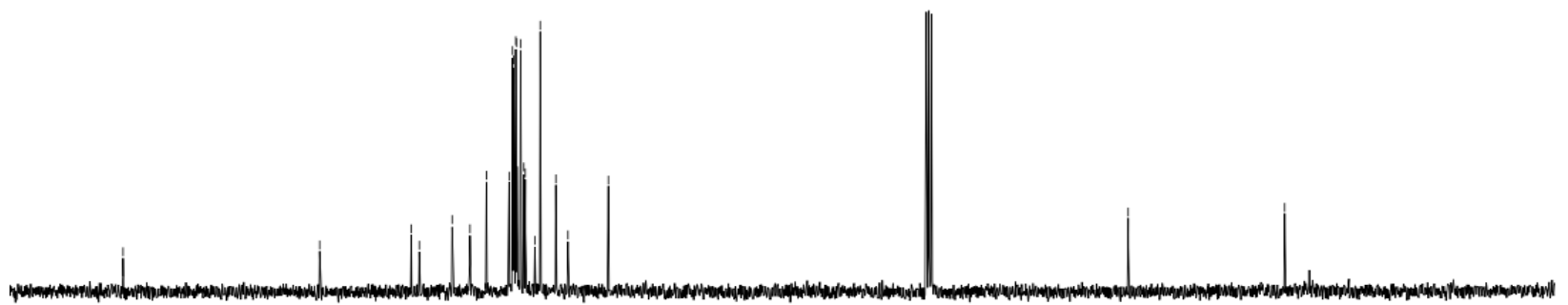

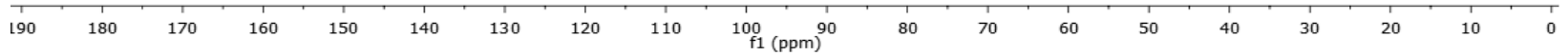




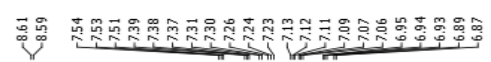

$\|\mid\|$ $\tilde{\bar{i}}$

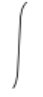

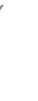

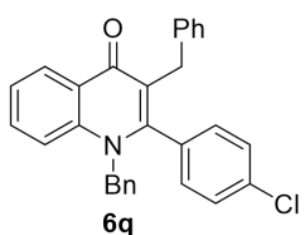

${ }^{1} \mathrm{H}$ NMR Spectrum (400 MHz, $\mathrm{CDCl}_{3}$ )

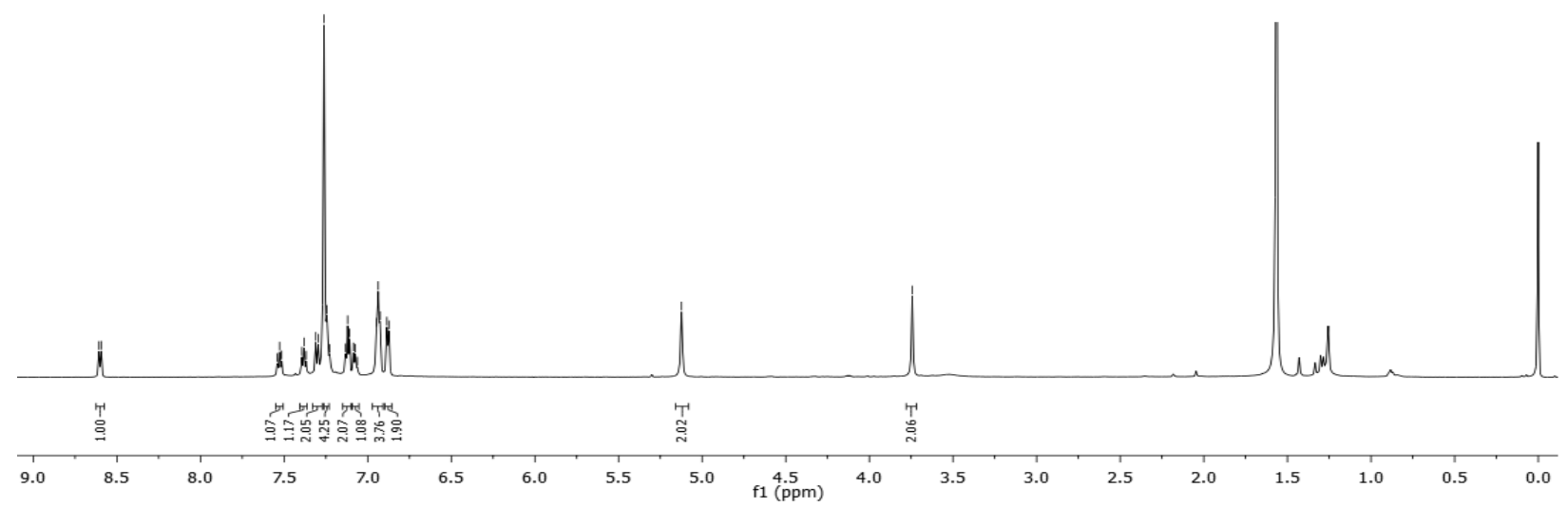

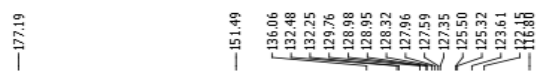
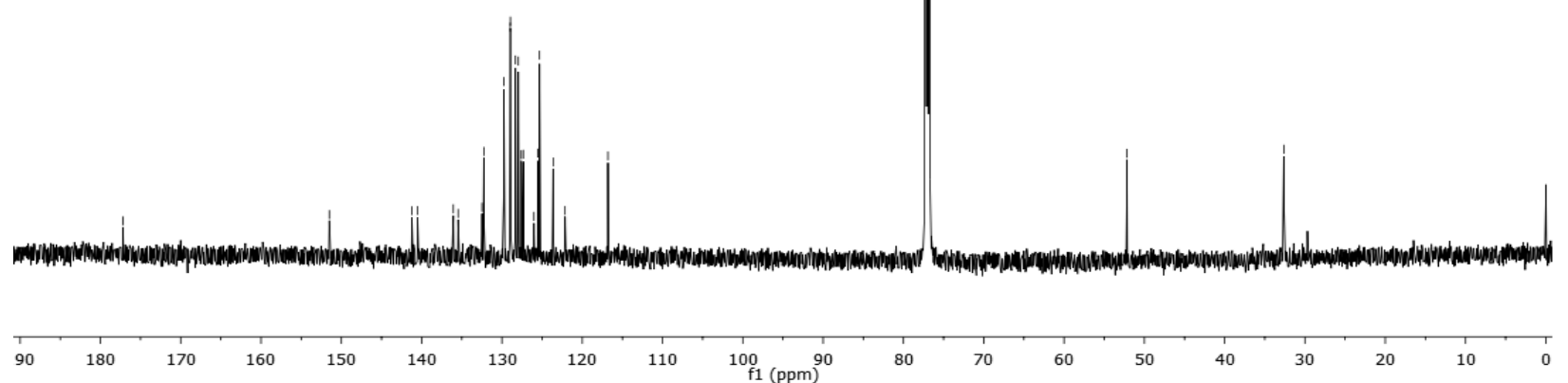


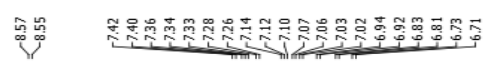

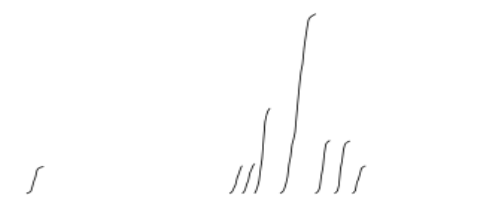

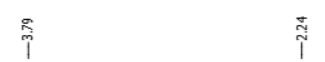
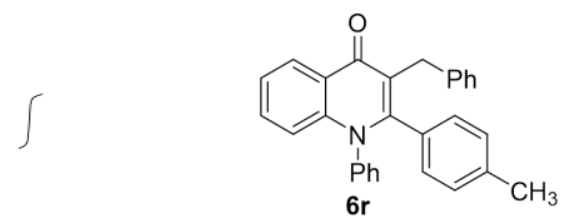

${ }^{1} \mathrm{H}$ NMR Spectrum

( $400 \mathrm{MHz}, \mathrm{CDCl}_{3}$ )
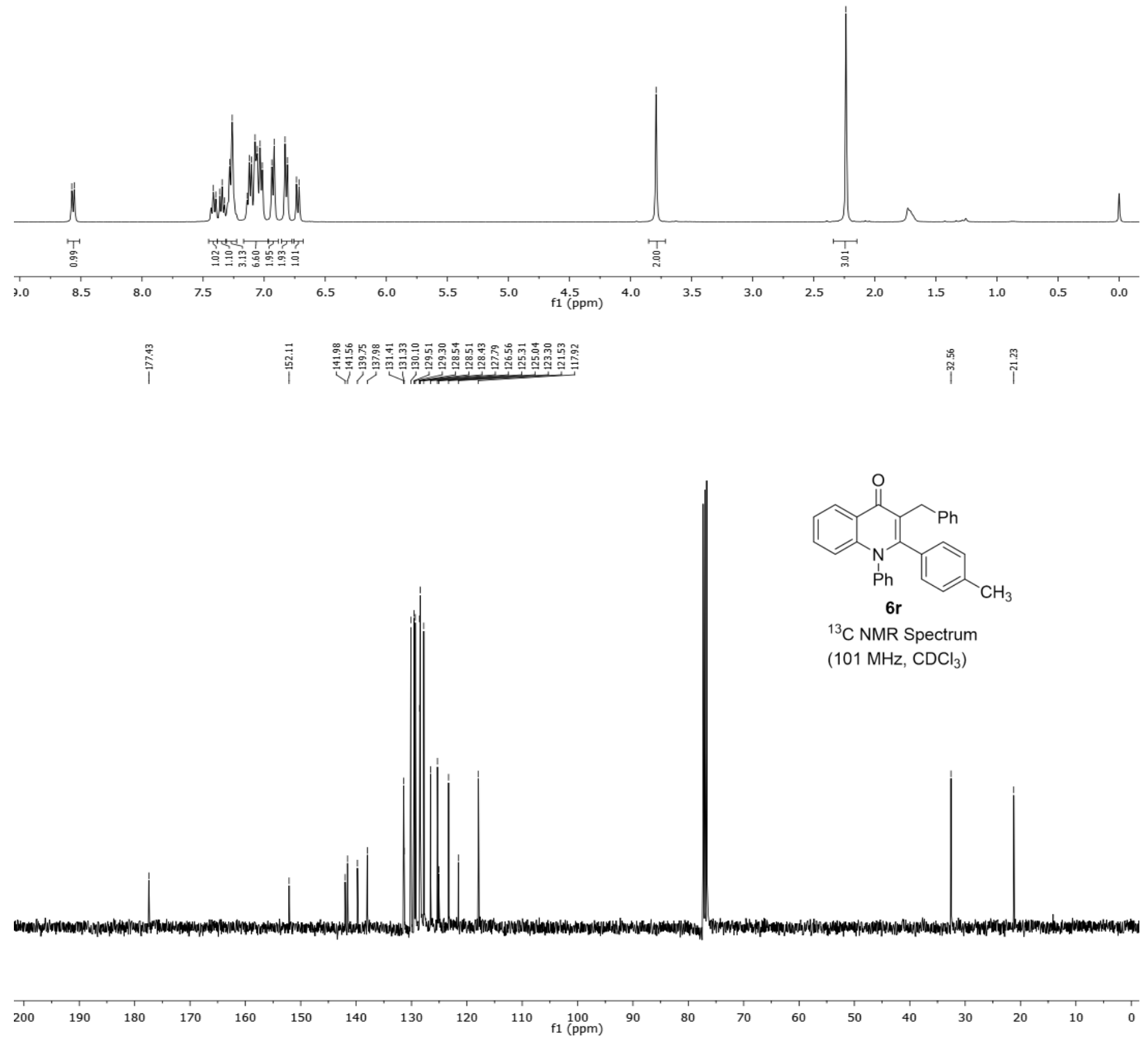

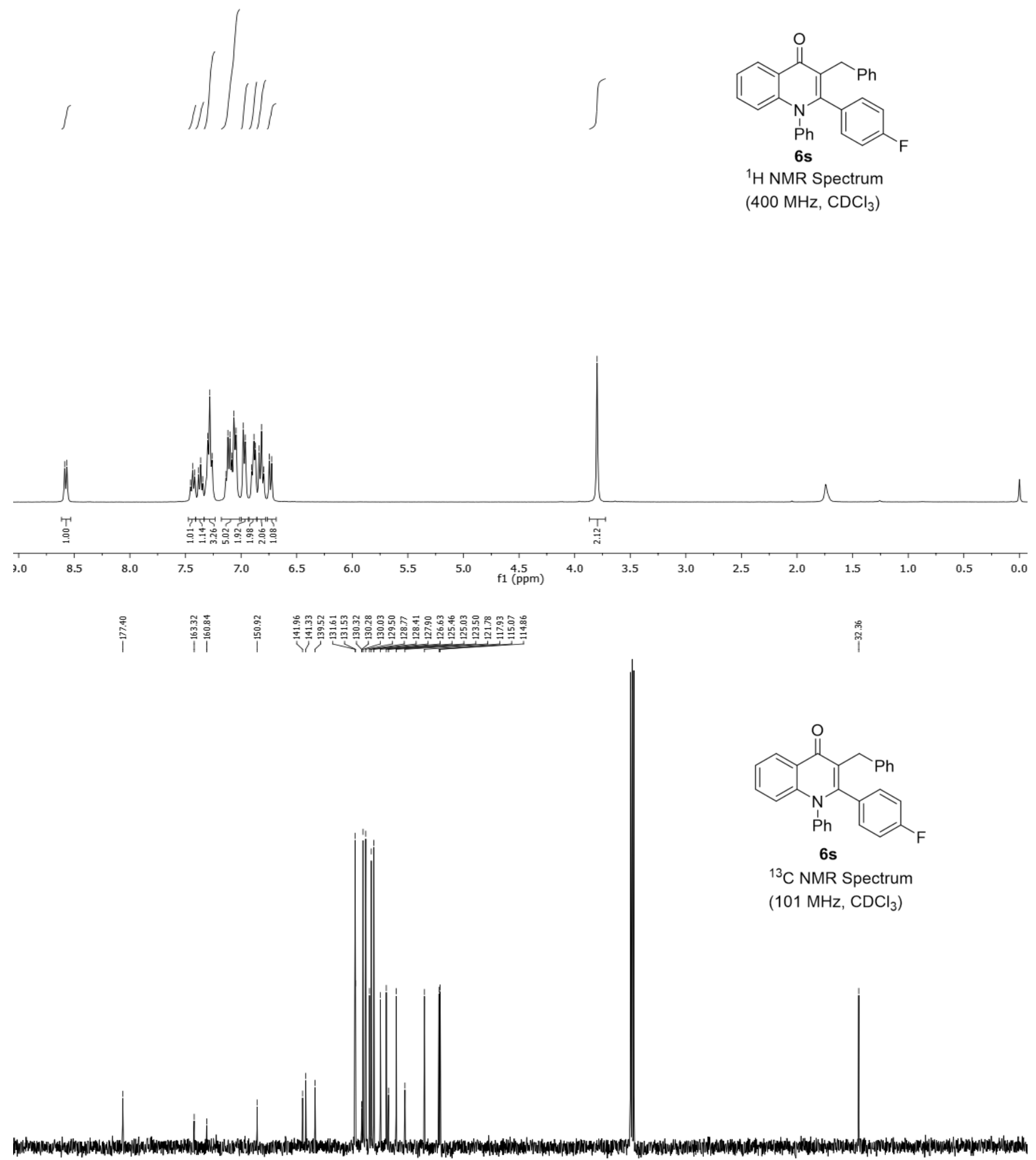


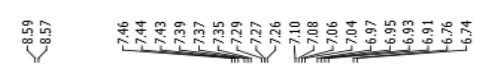

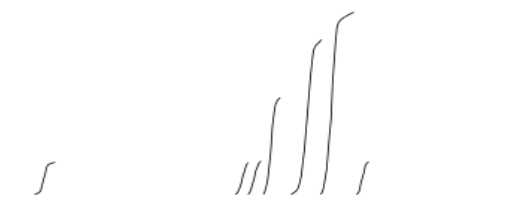

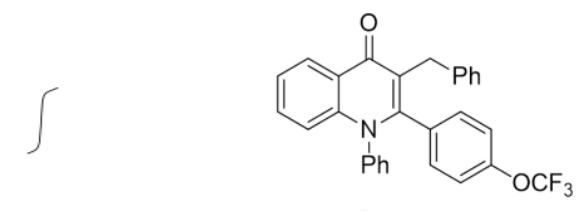

6u

${ }^{1} \mathrm{H}$ NMR Spectrum (400 MHz, $\mathrm{CDCl}_{3}$ )
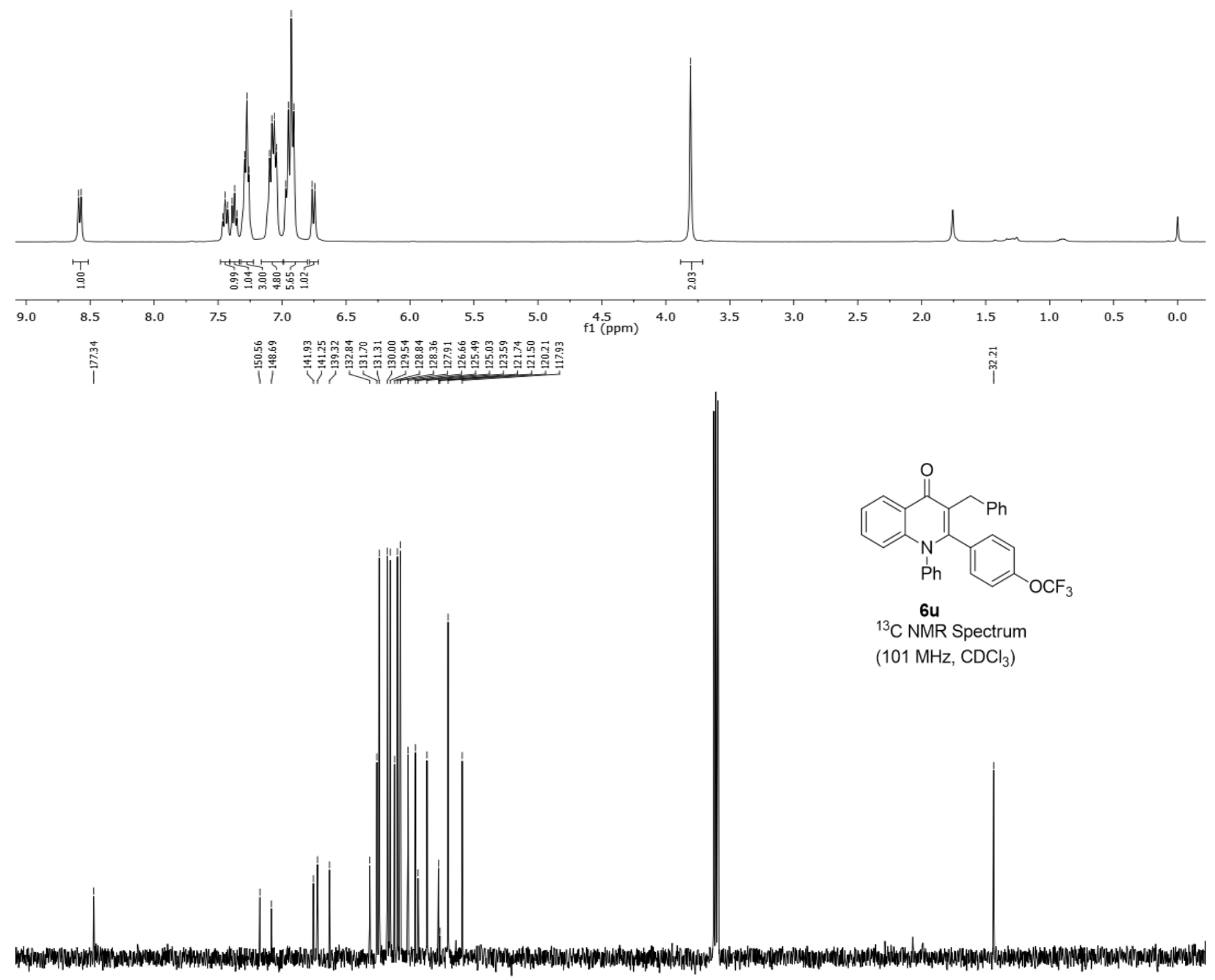

\begin{tabular}{|c|c|c|c|c|c|c|c|c|c|c|c|c|c|c|c|c|}
\hline 180 & 170 & 160 & 150 & 140 & 130 & 120 & 110 & $100 \mathrm{f1}(\mathrm{ppm})$ & 80 & 70 & 60 & 50 & 40 & 30 & 20 & 10 \\
\hline
\end{tabular}



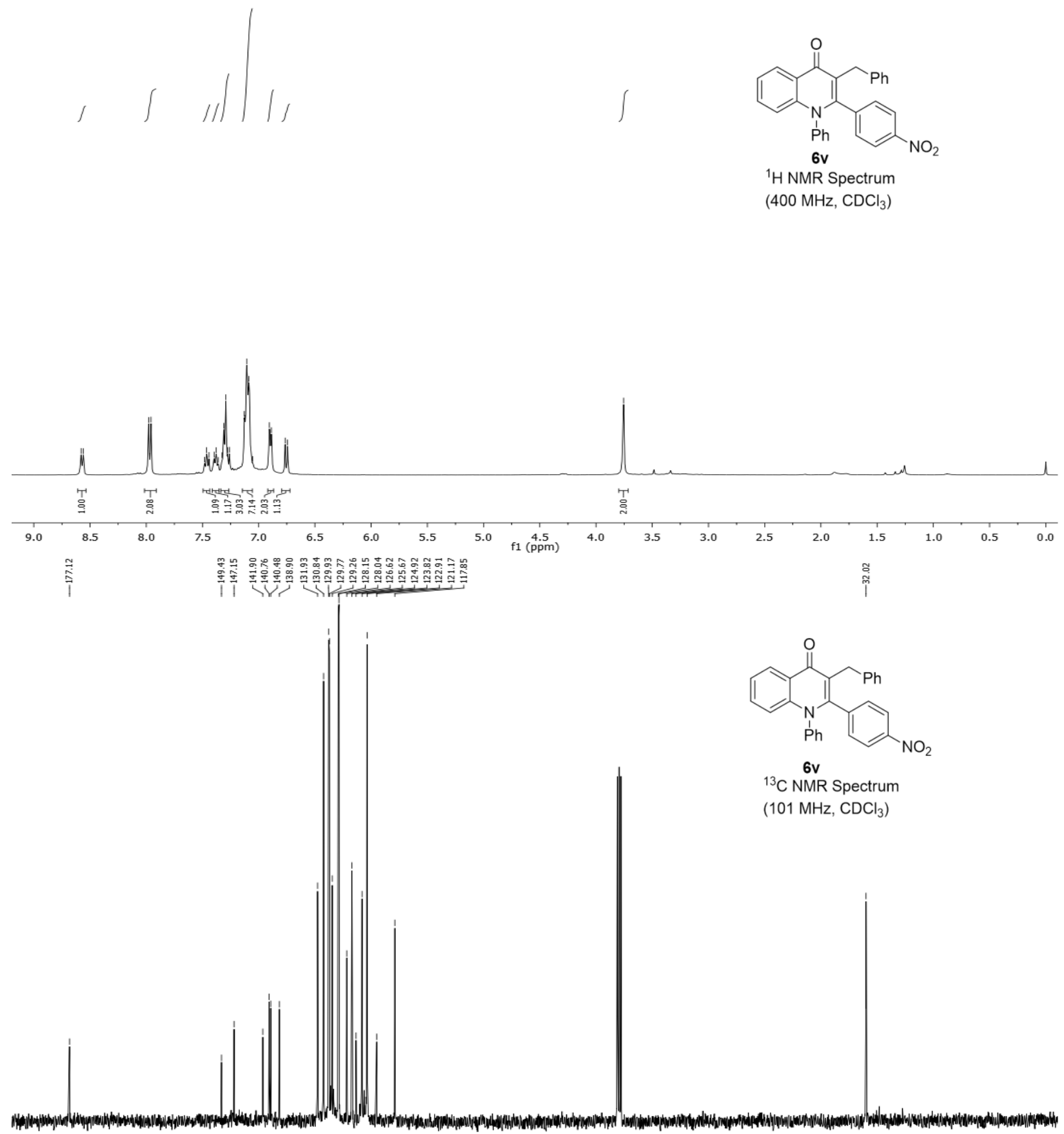

\begin{tabular}{|c|c|c|c|c|c|c|c|c|c|c|c|c|c|c|c|c|c|}
\hline 180 & 170 & 160 & 150 & 140 & 130 & 120 & 110 & 100 & $=1\left(\begin{array}{c}90 \\
\mathrm{ppm}\end{array}\right)$ & 80 & 70 & 60 & 50 & 40 & 30 & 20 & 10 \\
\hline
\end{tabular}




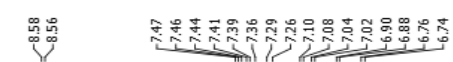

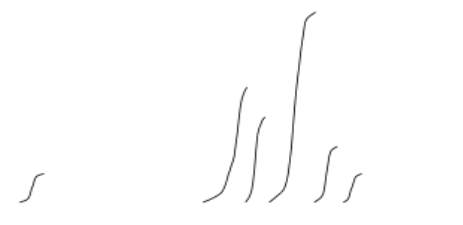

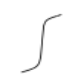

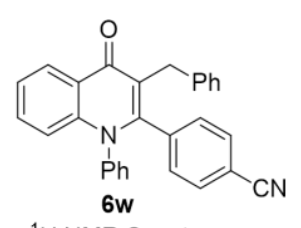

${ }^{1} \mathrm{H}$ NMR Spectrum (400 MHz, $\mathrm{CDCl}_{3}$ )
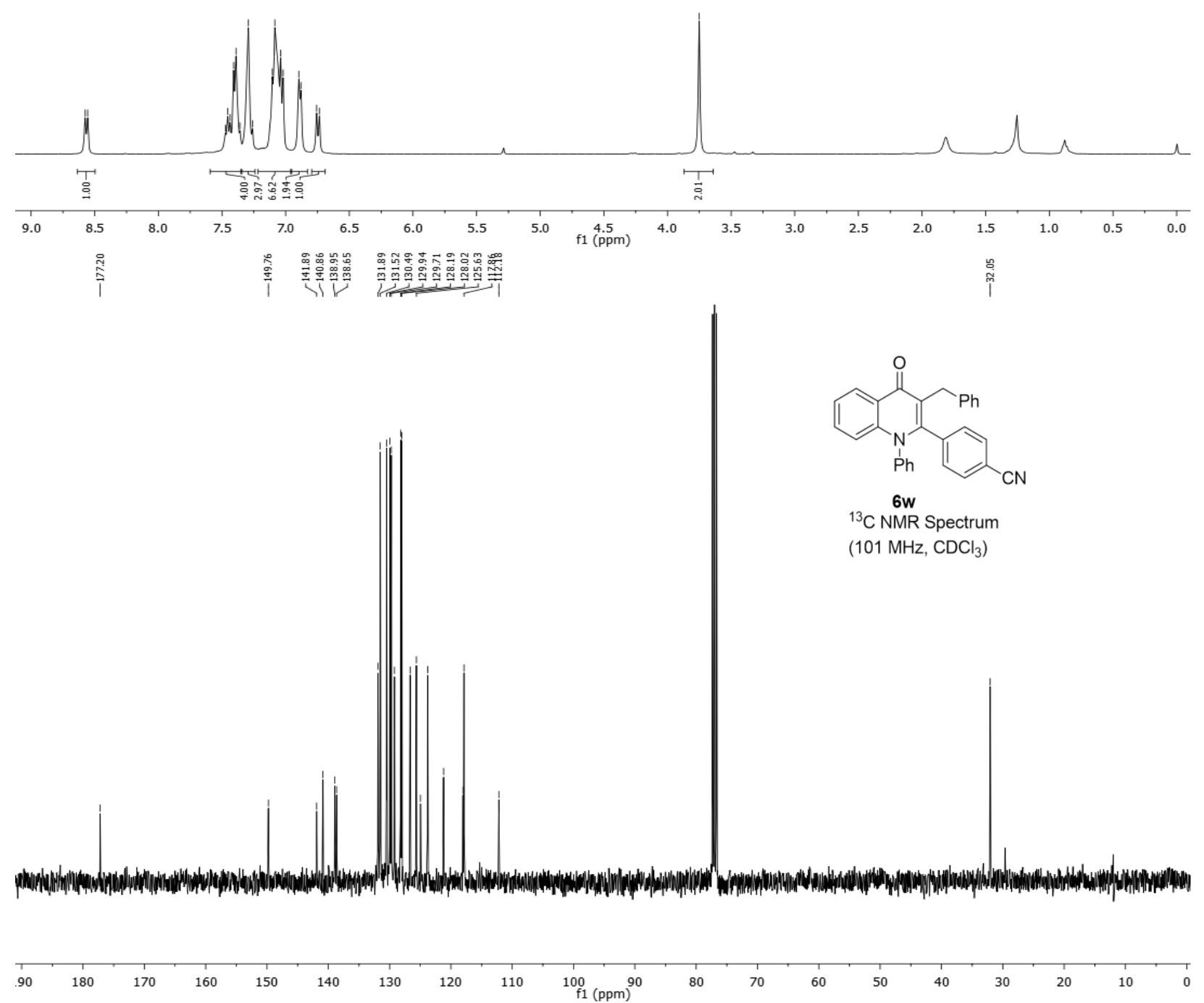


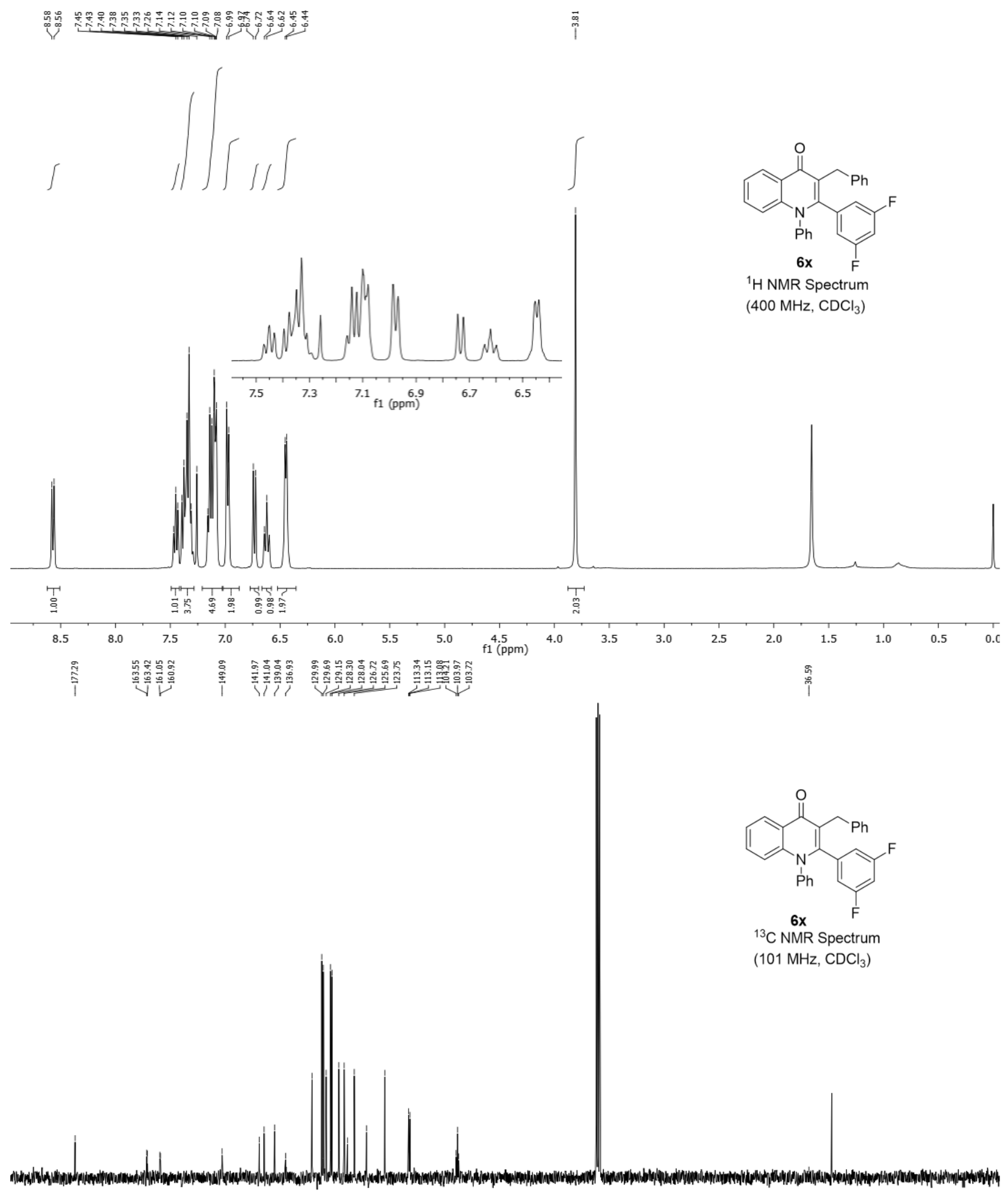

\begin{tabular}{|c|c|c|c|c|c|c|c|c|c|c|c|c|c|c|c|c|}
\hline 180 & 170 & 160 & 150 & 140 & 130 & 120 & 110 & ${ }_{\mathrm{f} 1(\mathrm{ppm})}^{90}$ & 80 & 70 & 60 & 50 & 40 & 30 & 20 & 10 \\
\hline
\end{tabular}




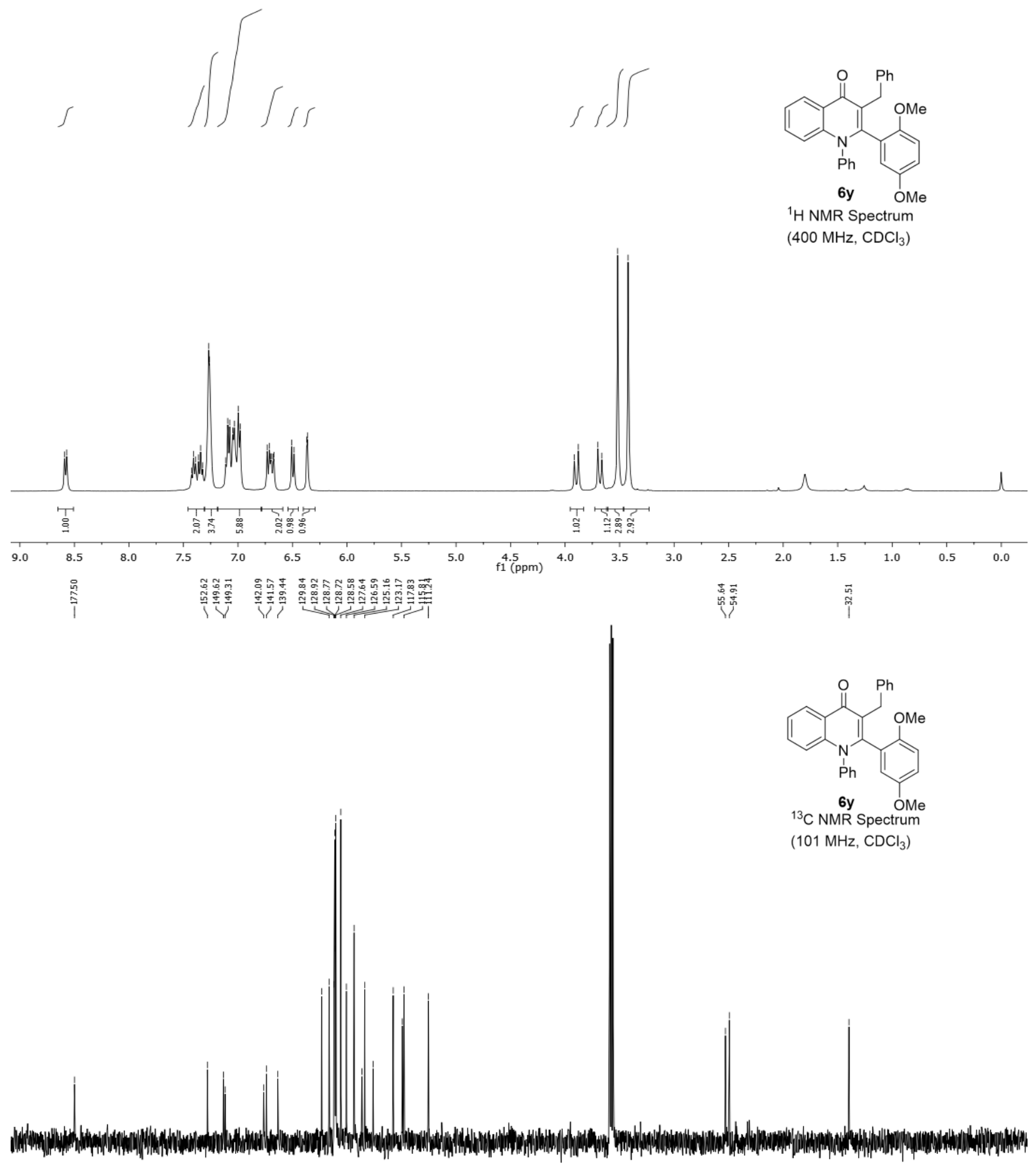

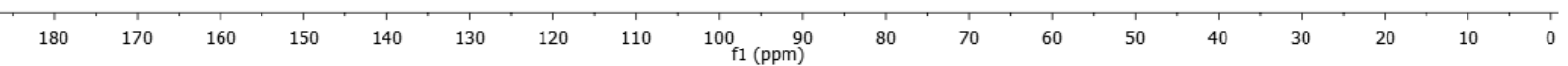




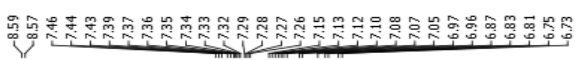

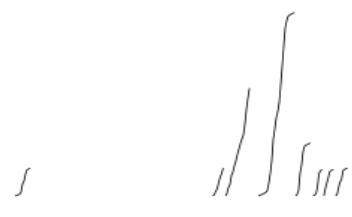

If

${ }^{1} \mathrm{H}$ NMR Spectrum

$\left(400 \mathrm{MHz}, \mathrm{CDCl}_{3}\right.$ )
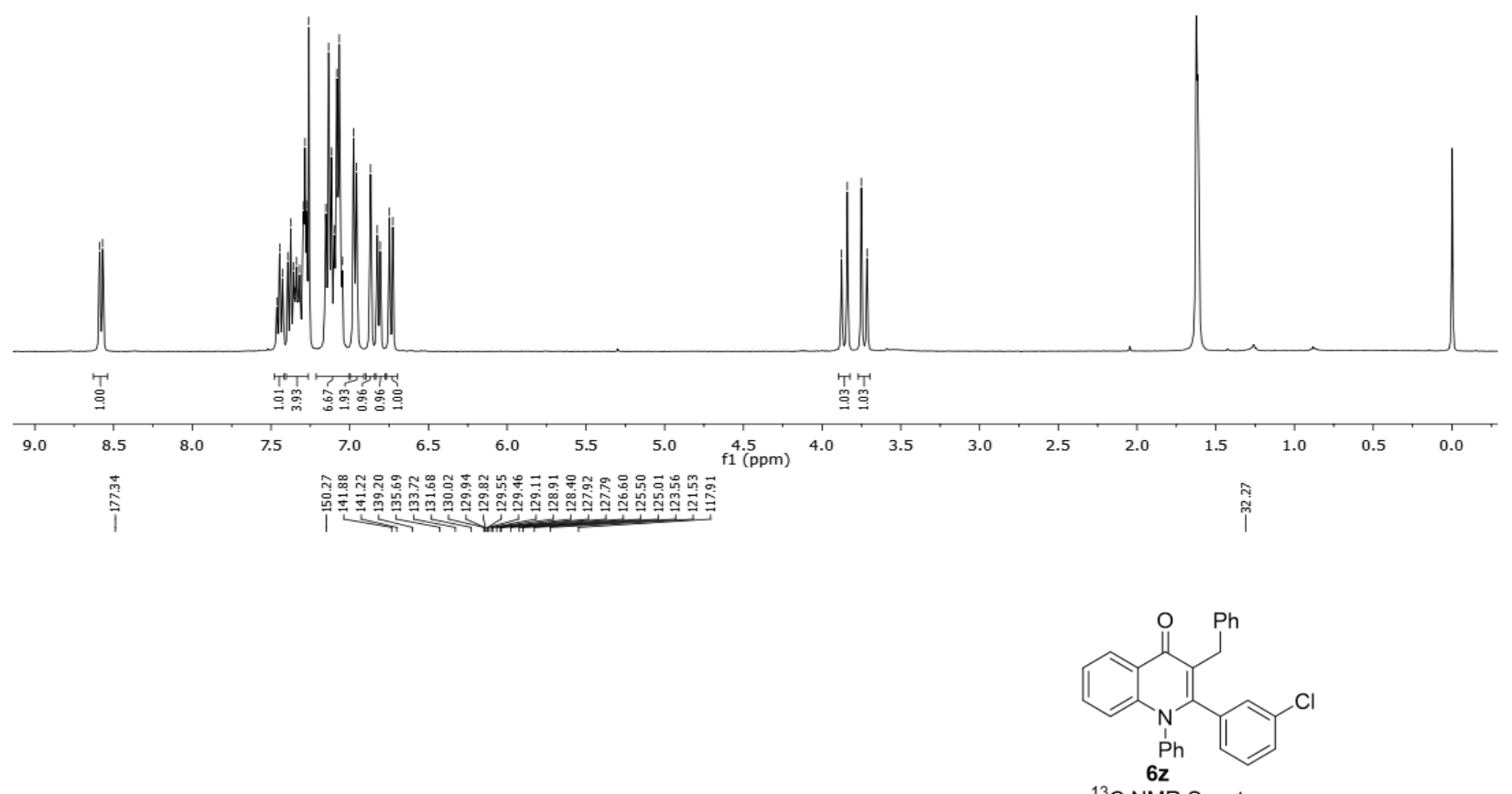

${ }^{13} \mathrm{C}$ NMR Spectrum
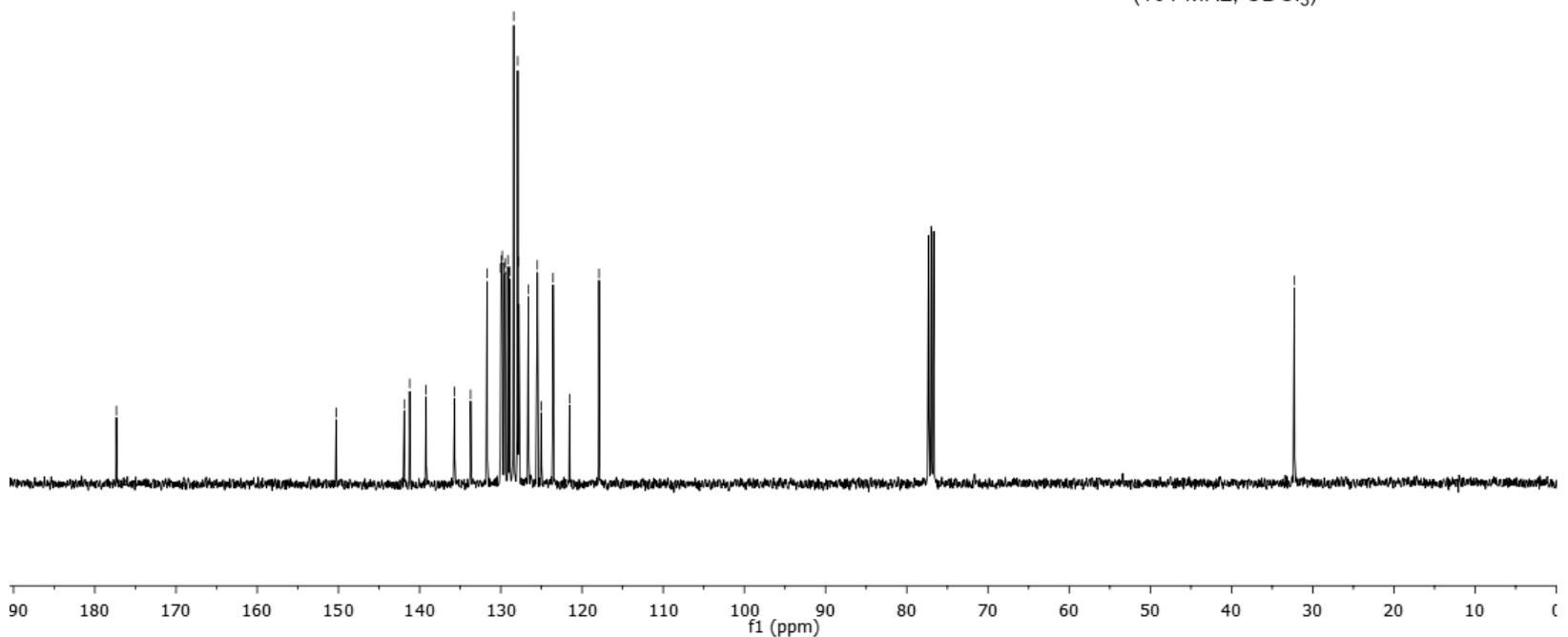

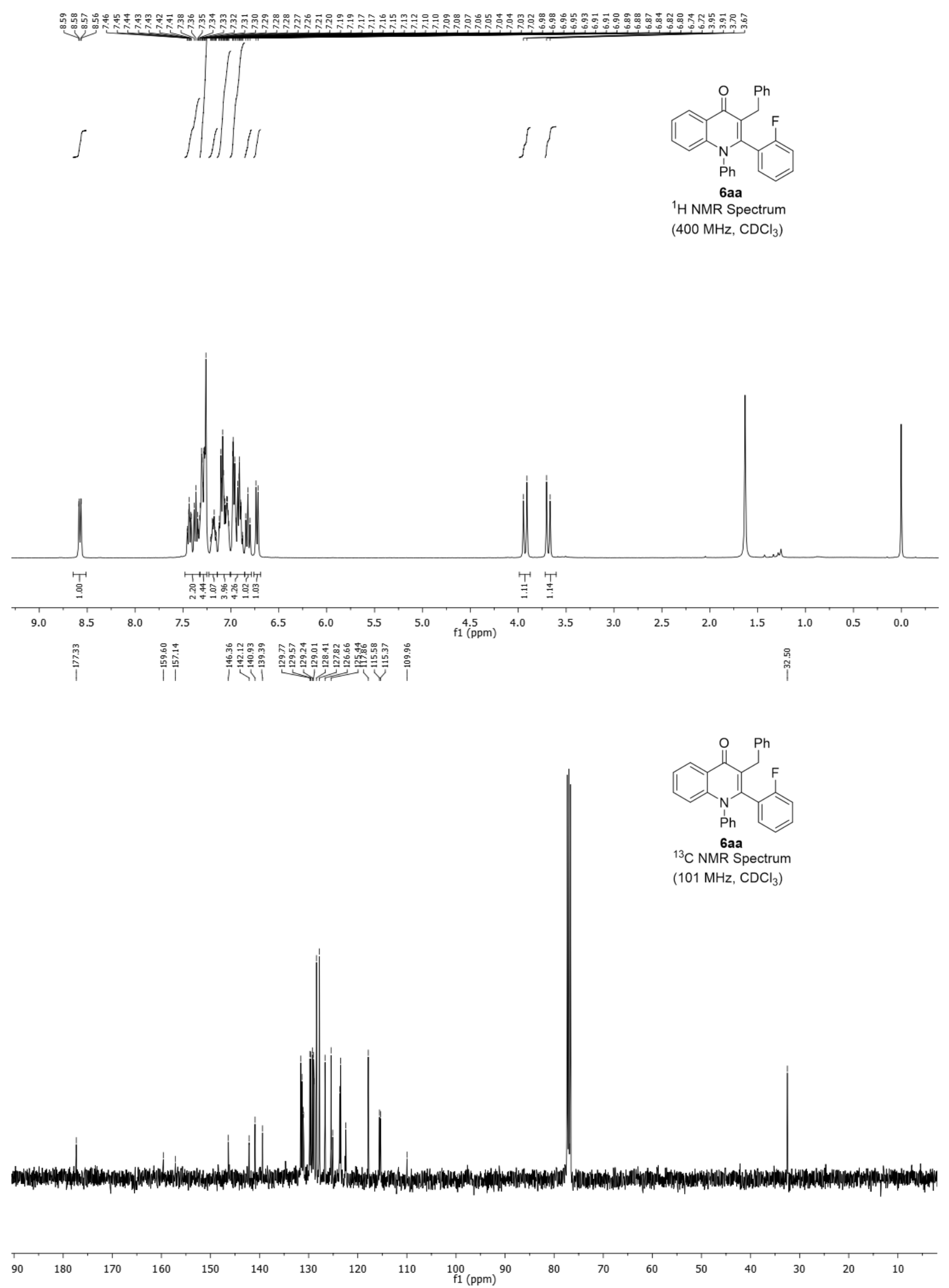


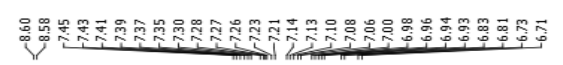

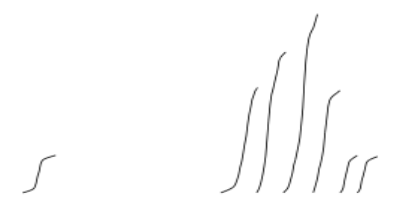

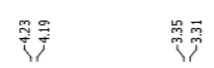

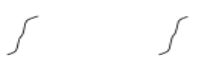

\}

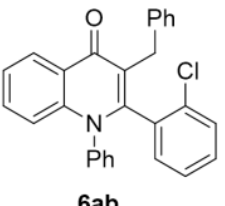

${ }^{1} \mathrm{H}$ NMR Spectrum $\left(400 \mathrm{MHz}, \mathrm{CDCl}_{3}\right.$ )
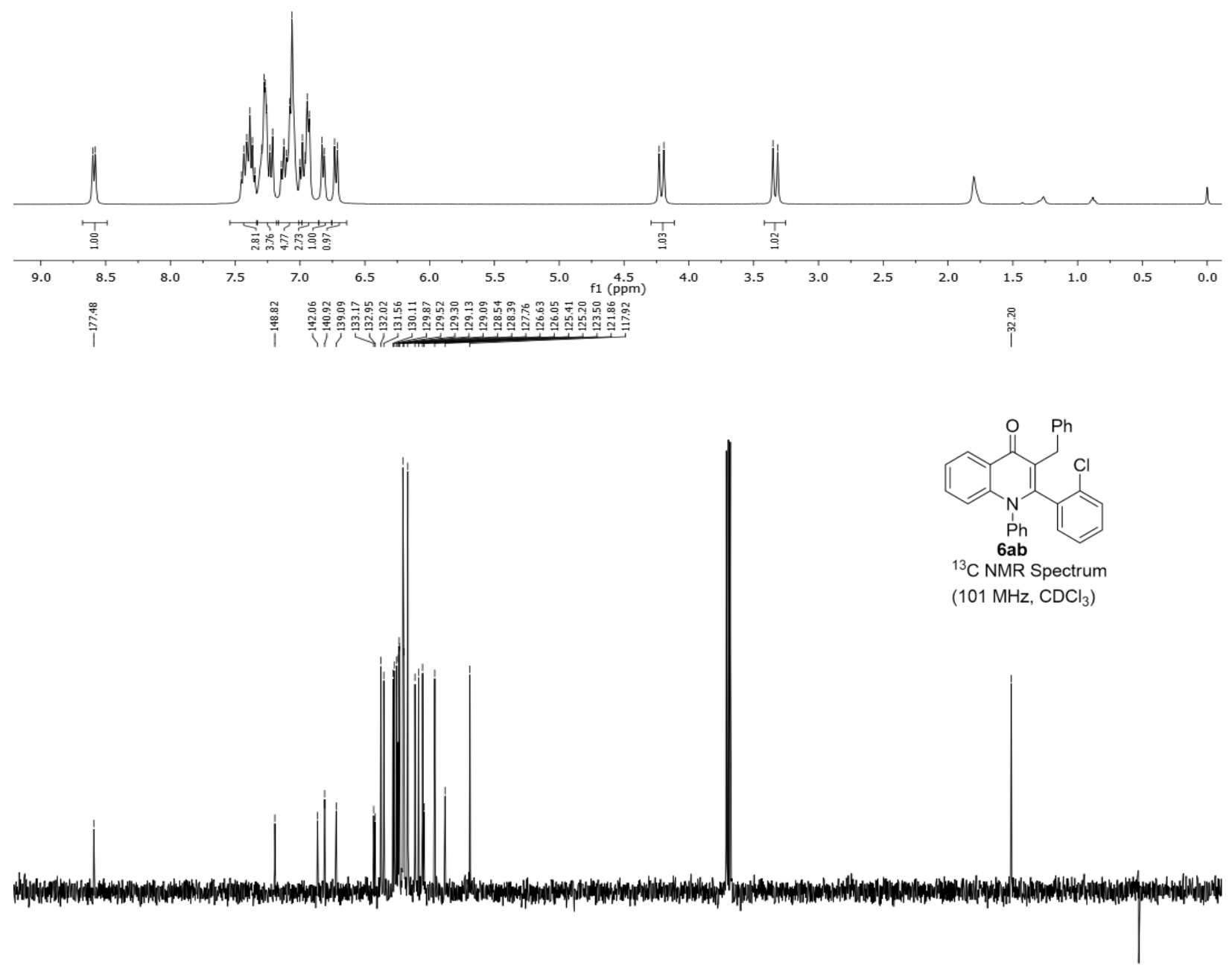

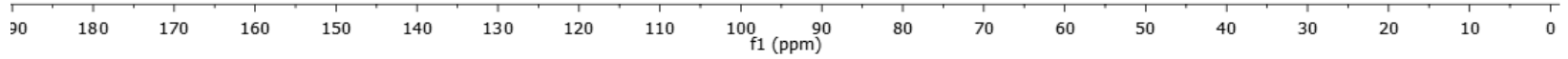



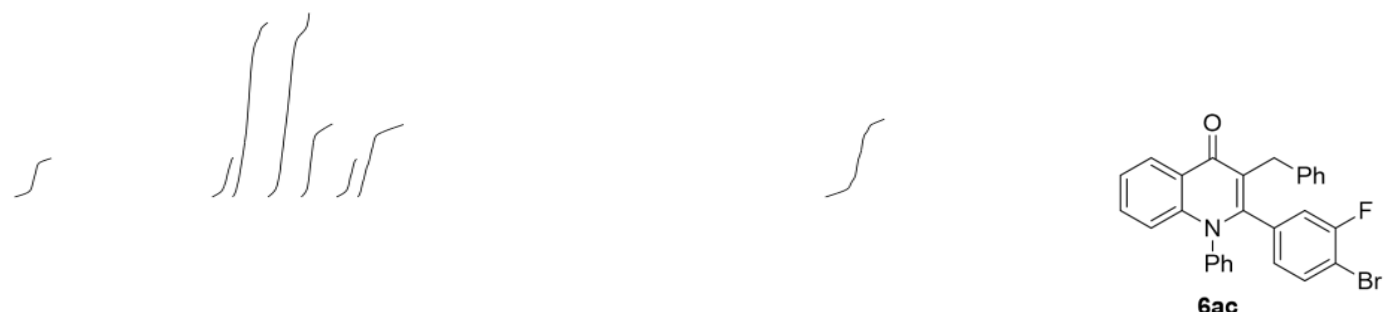

${ }^{1} \mathrm{H}$ NMR Spectrum $\left(400 \mathrm{MHz}, \mathrm{CDCl}_{3}\right)$
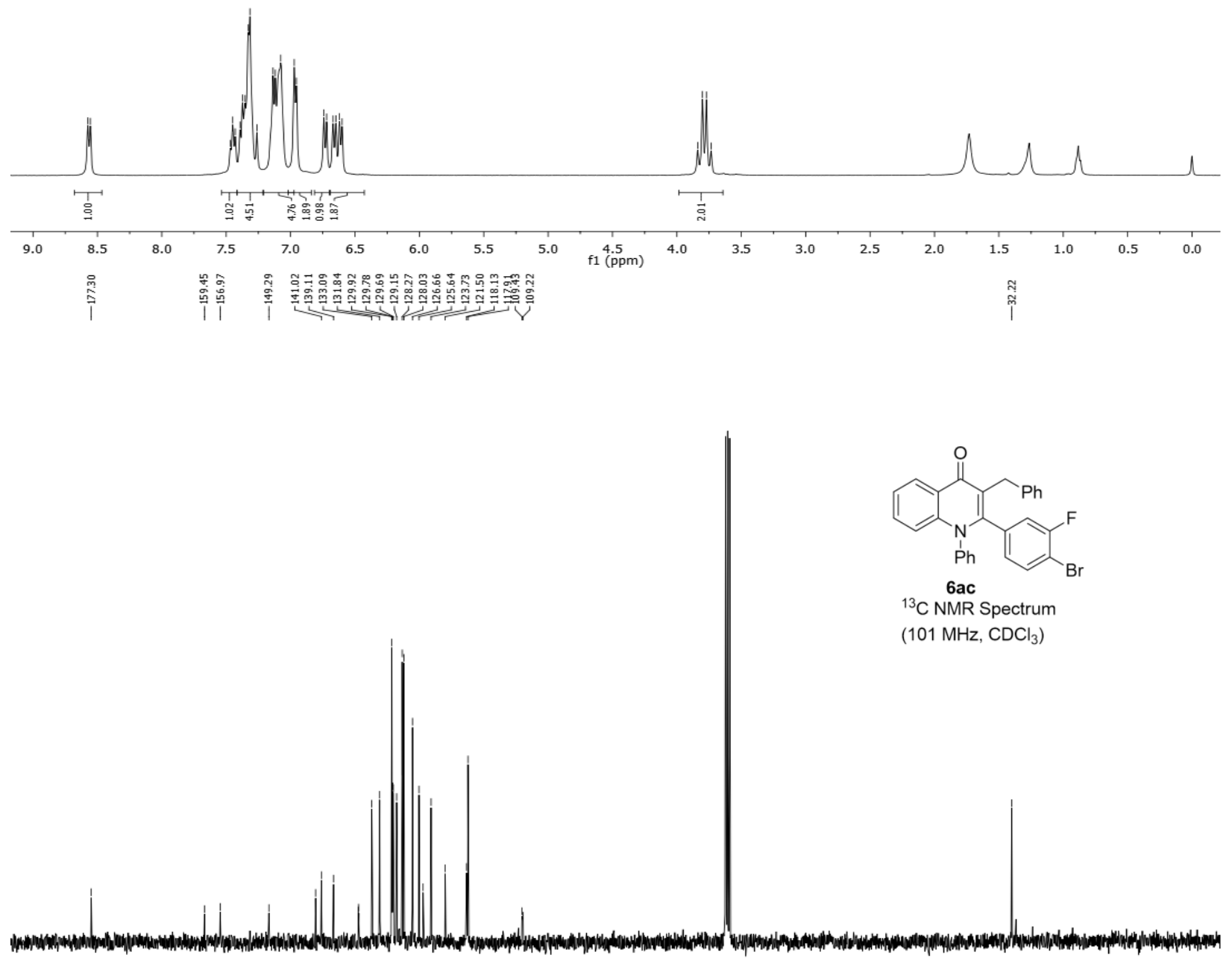

6 ac

${ }^{13} \mathrm{C}$ NMR Spectrum

$\left(101 \mathrm{MHz}, \mathrm{CDCl}_{3}\right)$

Mam

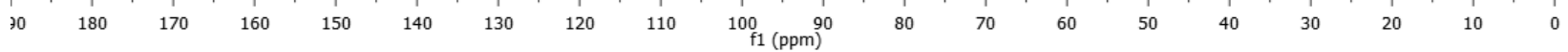




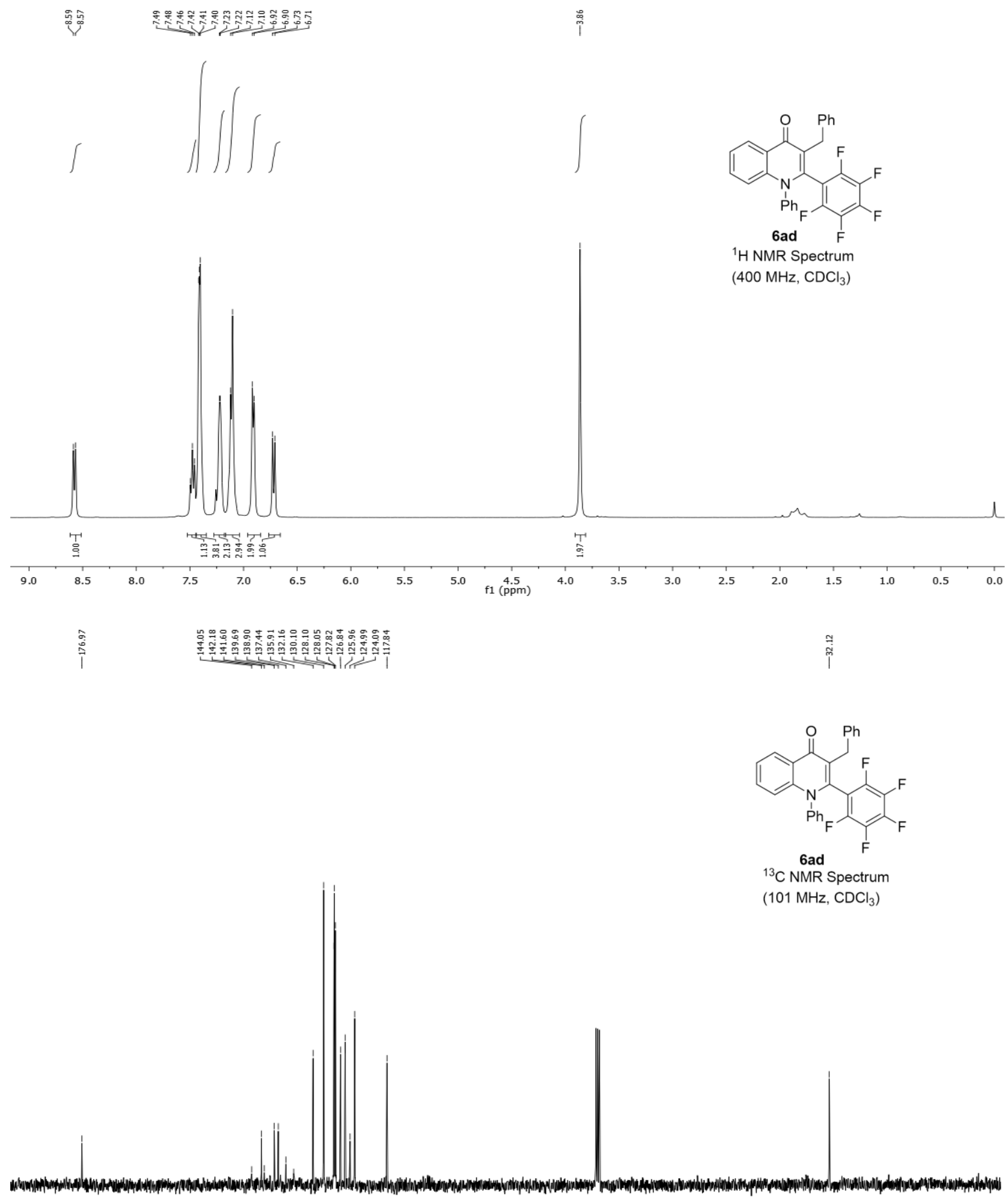

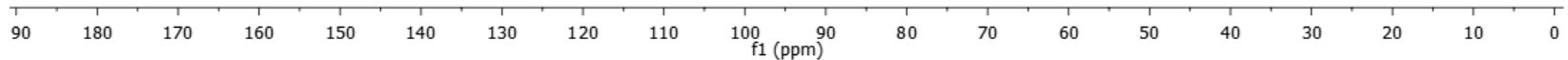



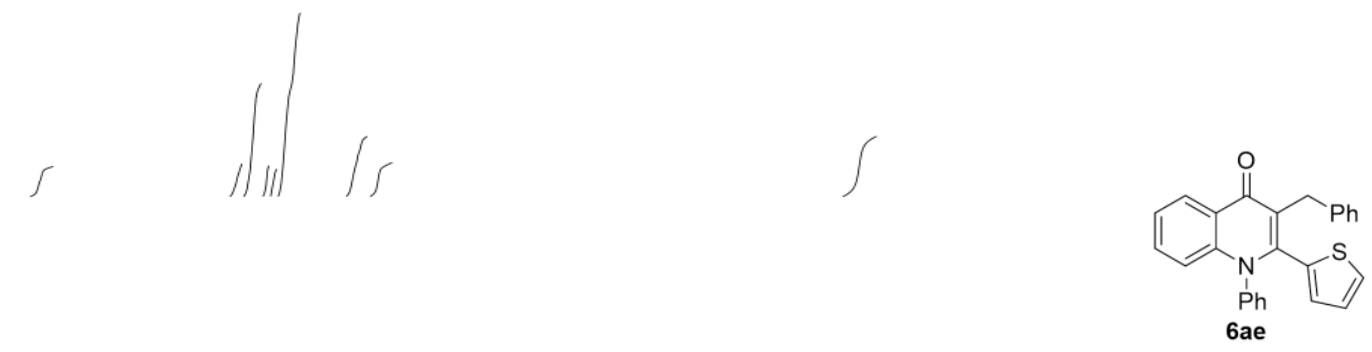

${ }^{1} \mathrm{H}$ NMR Spectrum $\left(400 \mathrm{MHz}, \mathrm{CDCl}_{3}\right.$ )
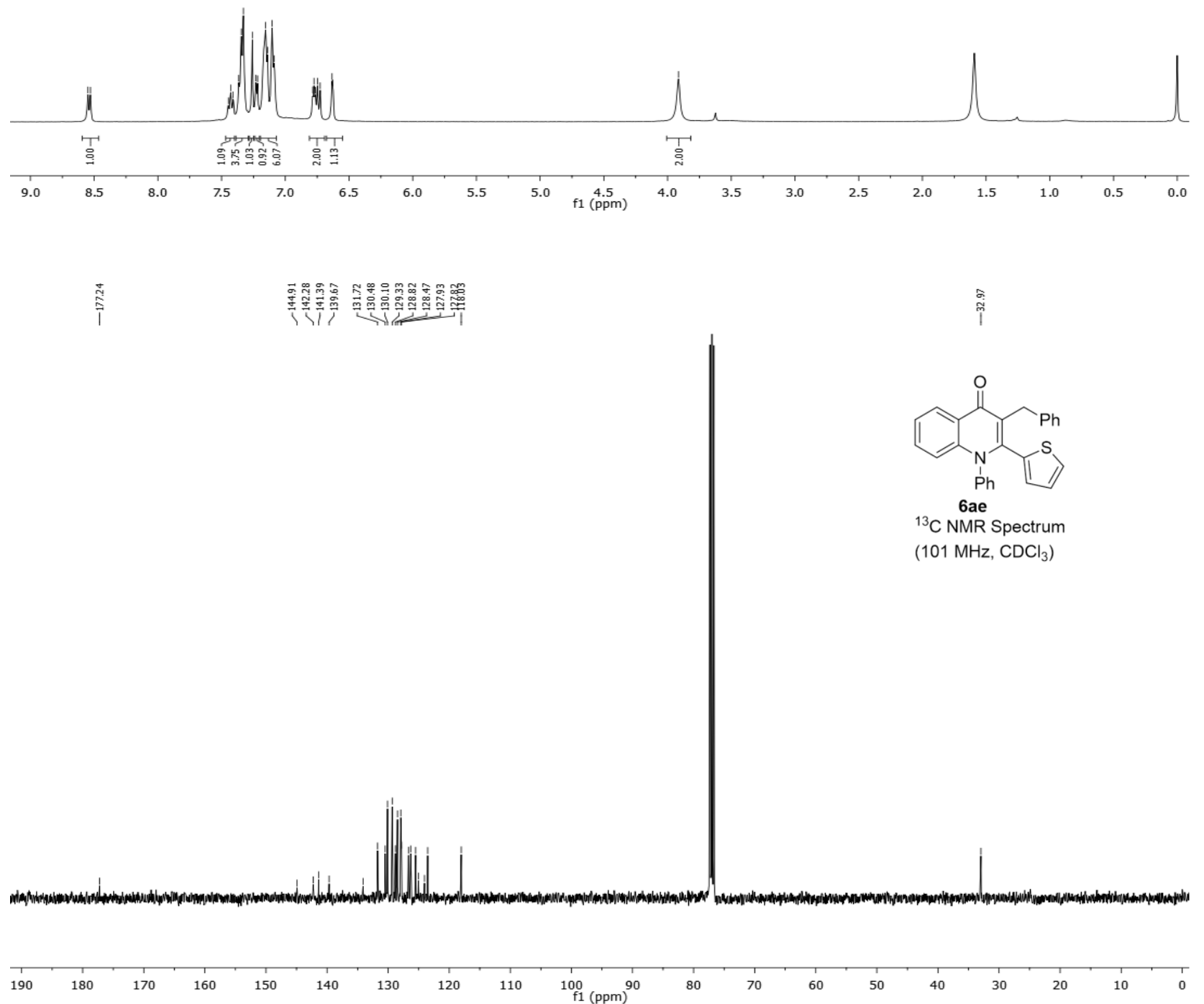

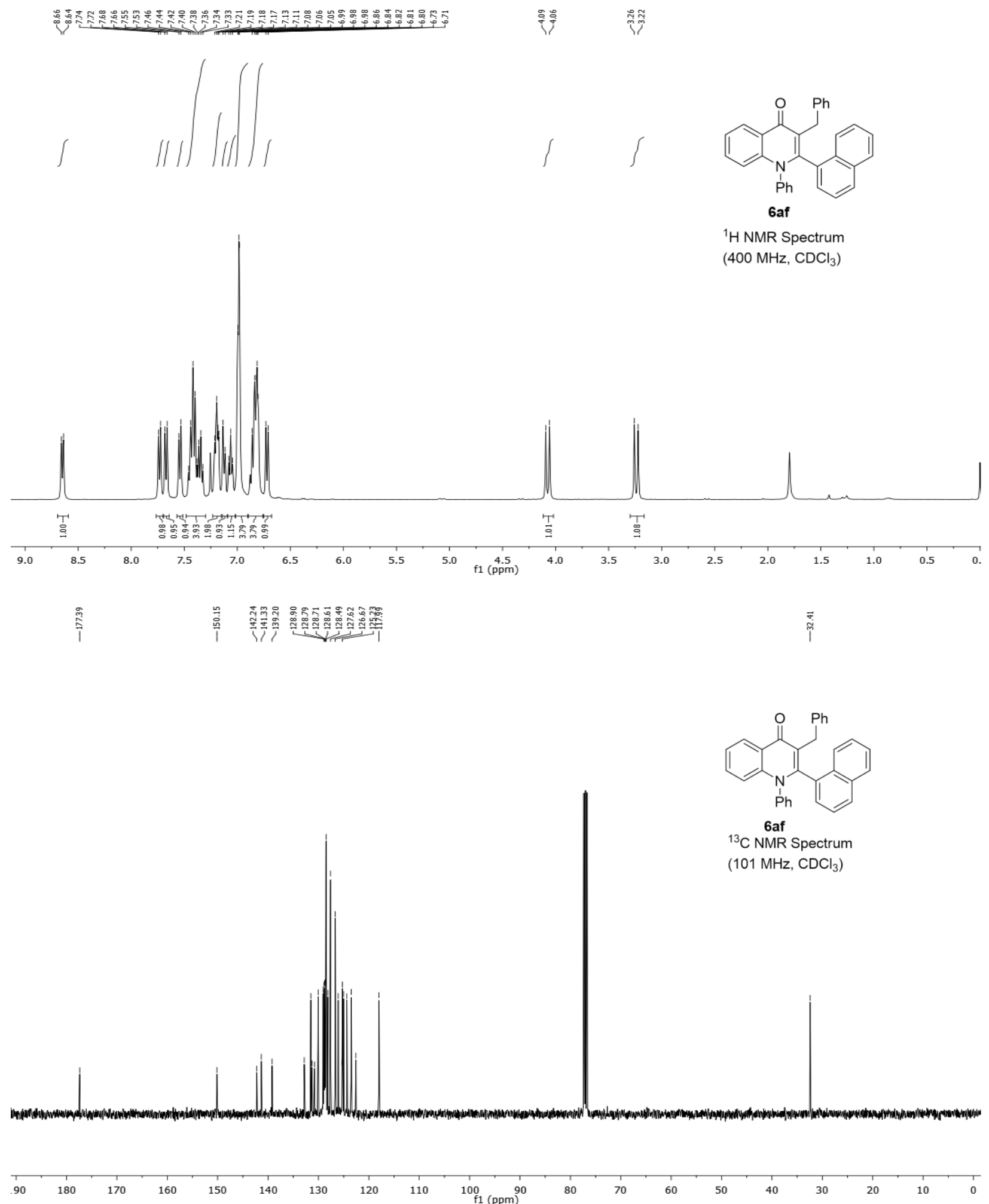

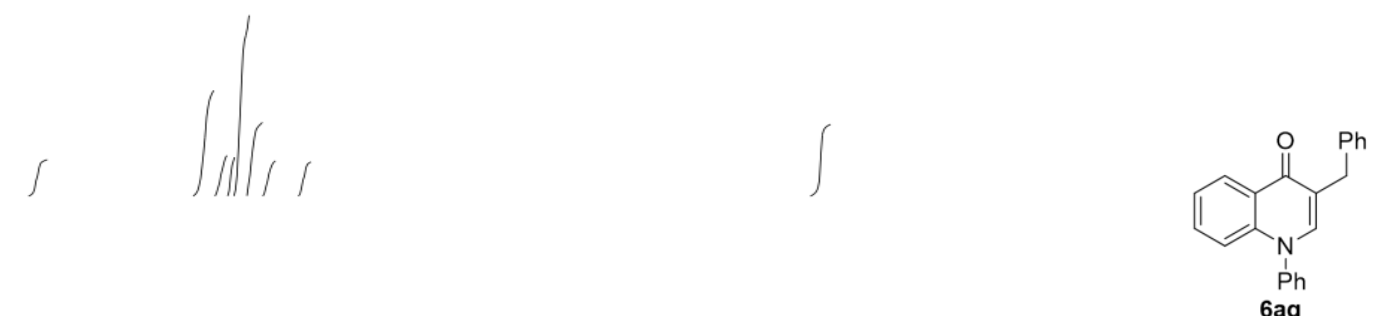

${ }^{1} \mathrm{H}$ NMR Spectrum

$\left(400 \mathrm{MHz}, \mathrm{CDCl}_{3}\right)$
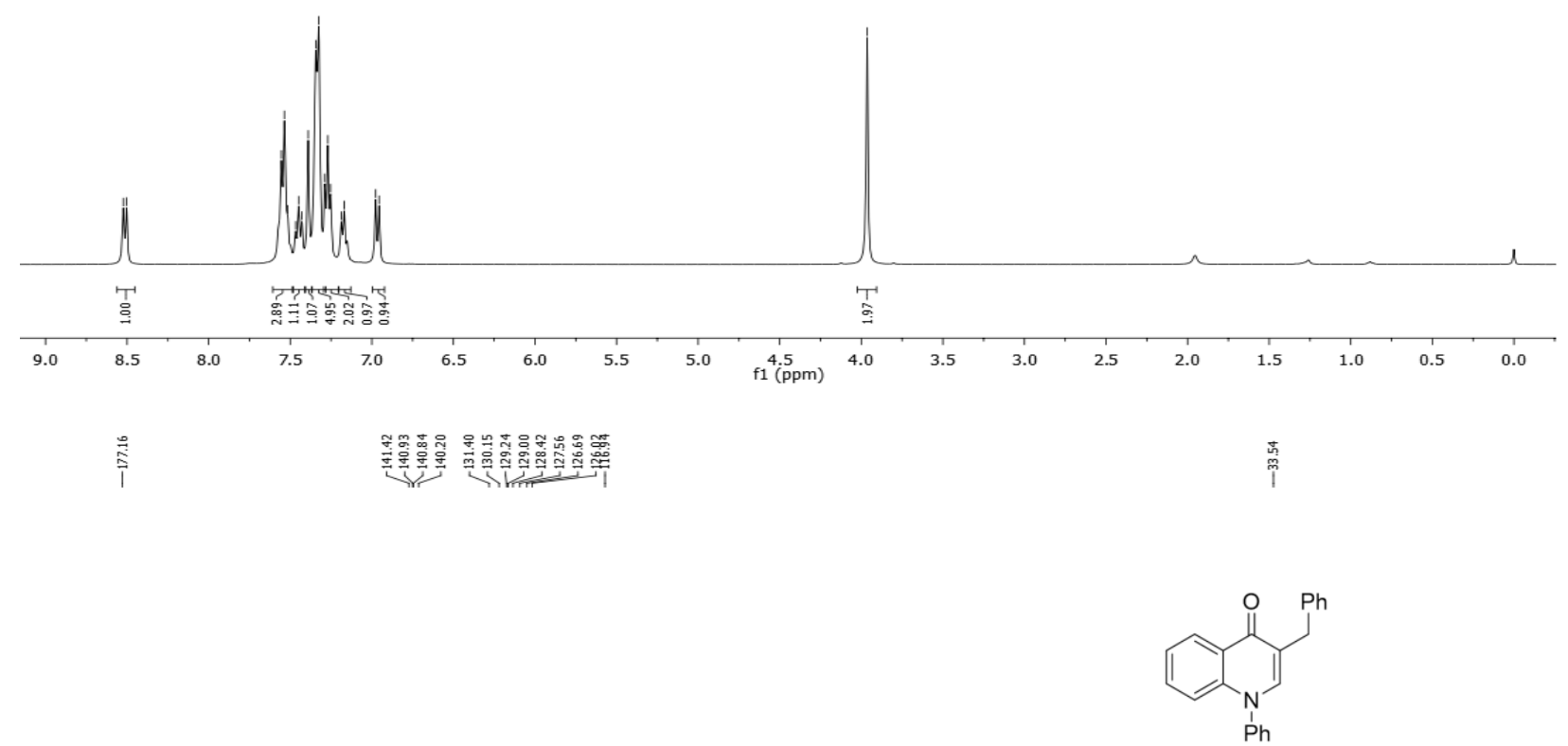

6ag

${ }^{13} \mathrm{C}$ NMR Spectrum

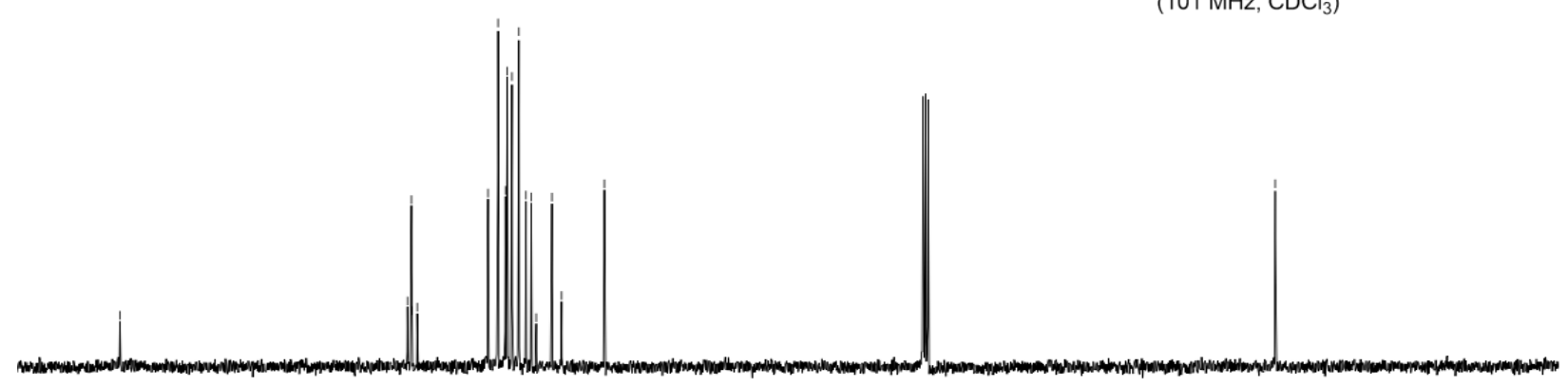

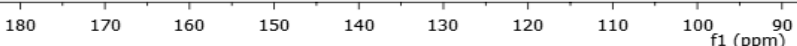



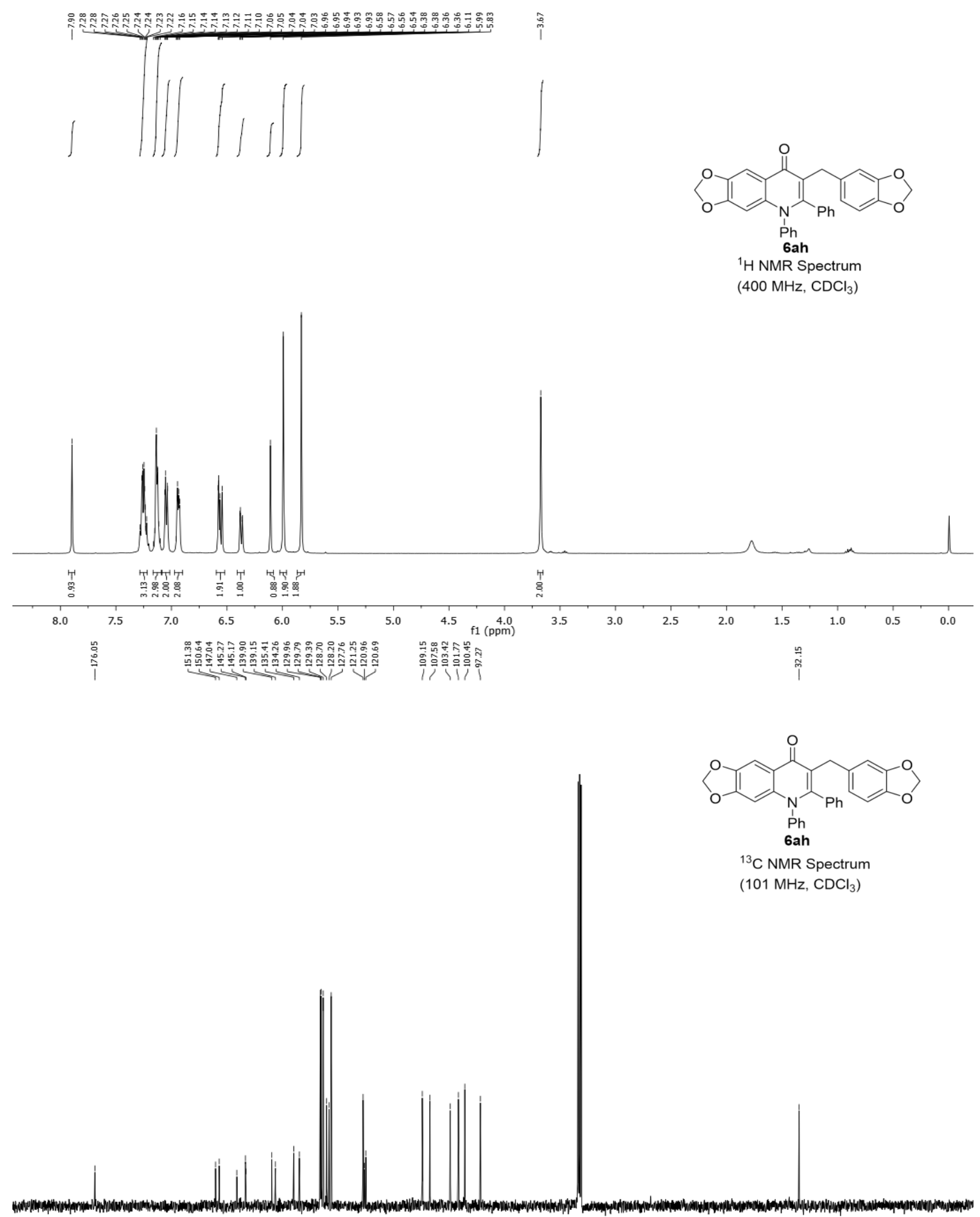

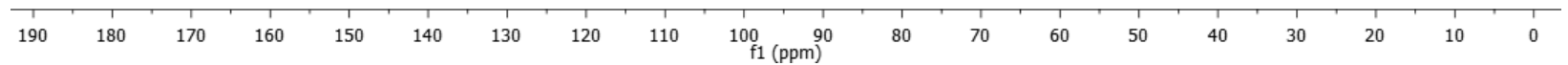




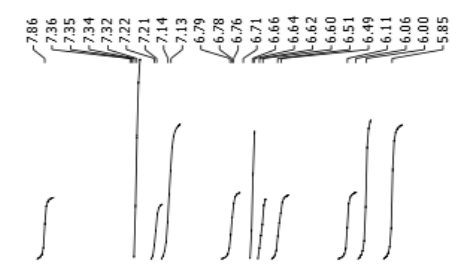

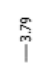

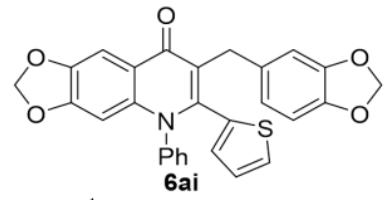

${ }^{1} \mathrm{H}$ NMR Spectrum $\left(400 \mathrm{MHz}, \mathrm{CDCl}_{3}\right)$
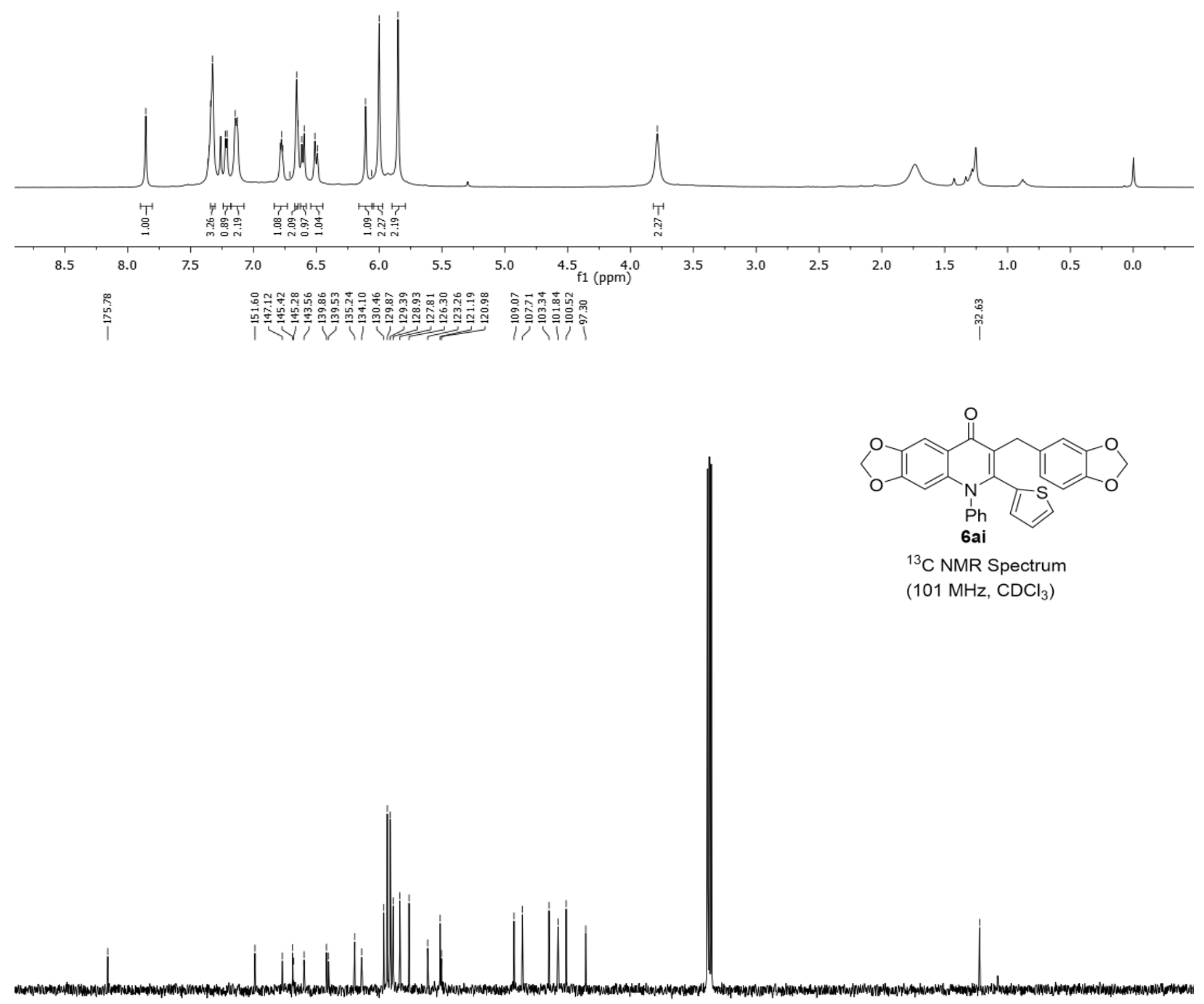

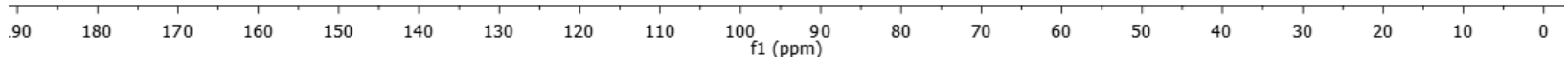



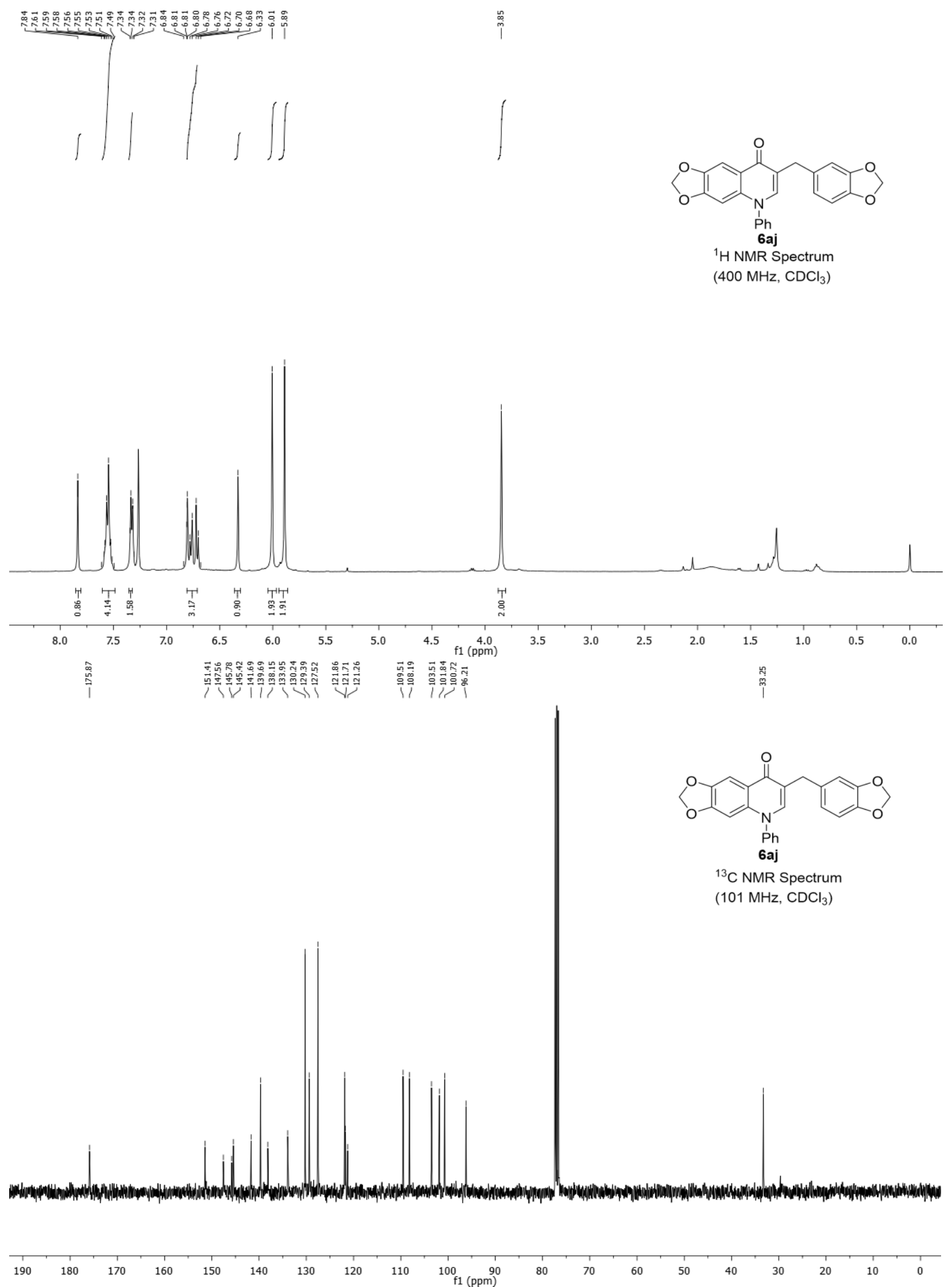

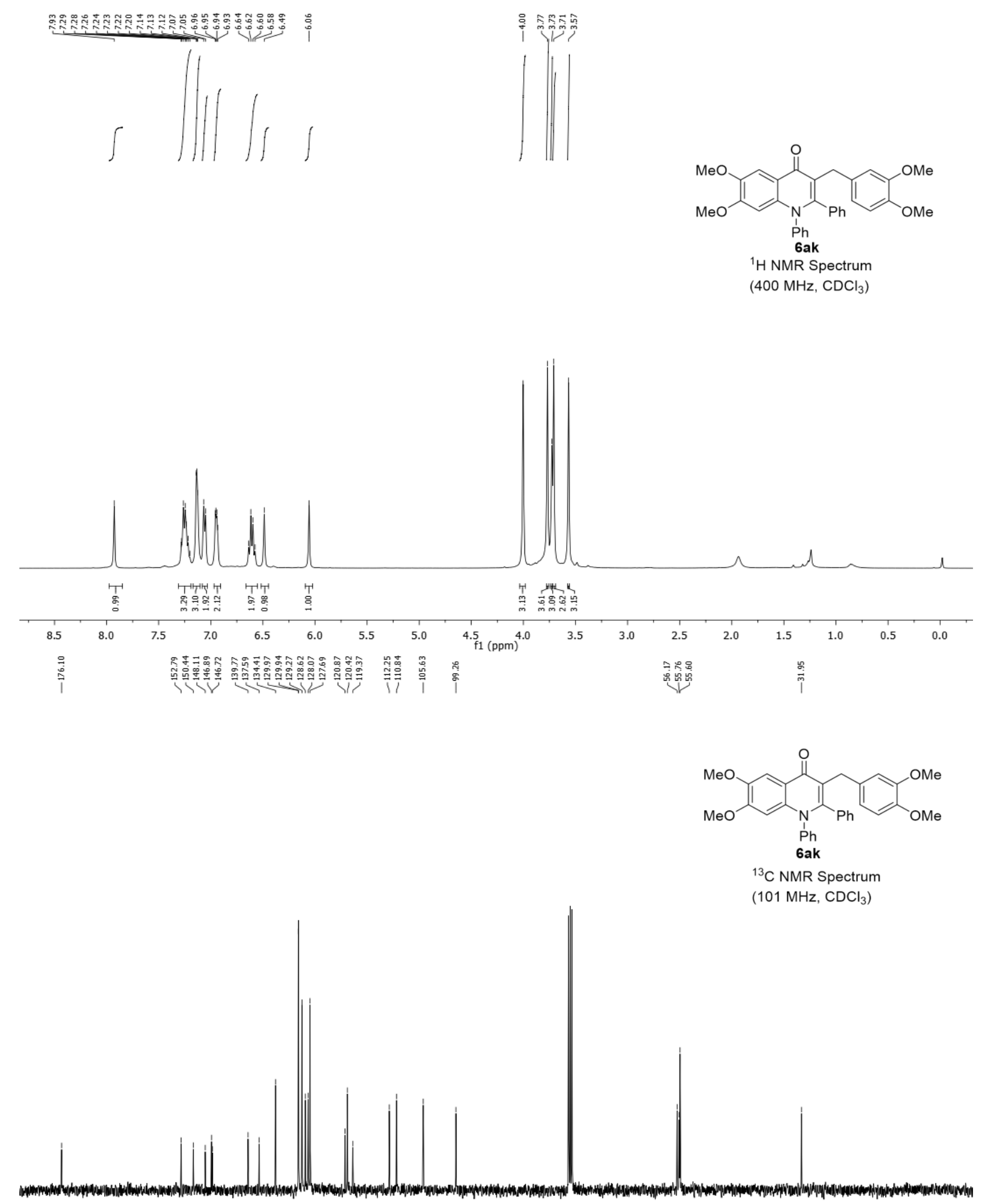

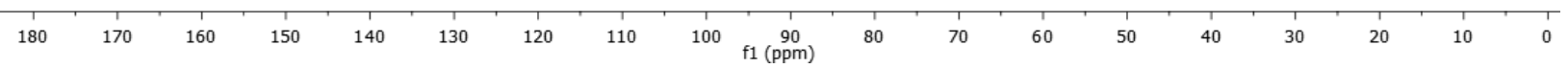



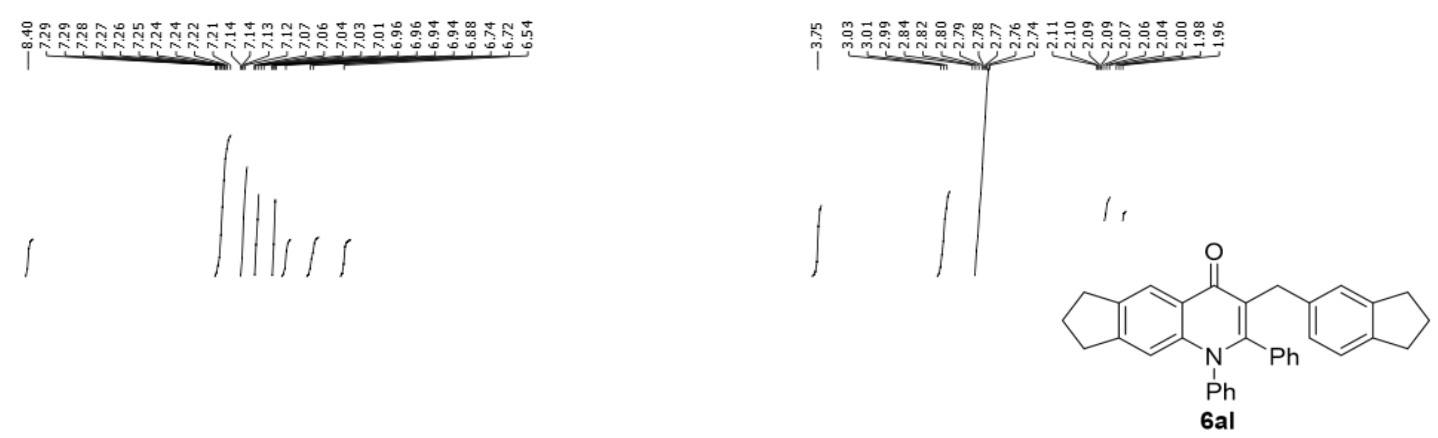

${ }^{1} \mathrm{H}$ NMR Spectrum (400 MHz, $\mathrm{CDCl}_{3}$ )
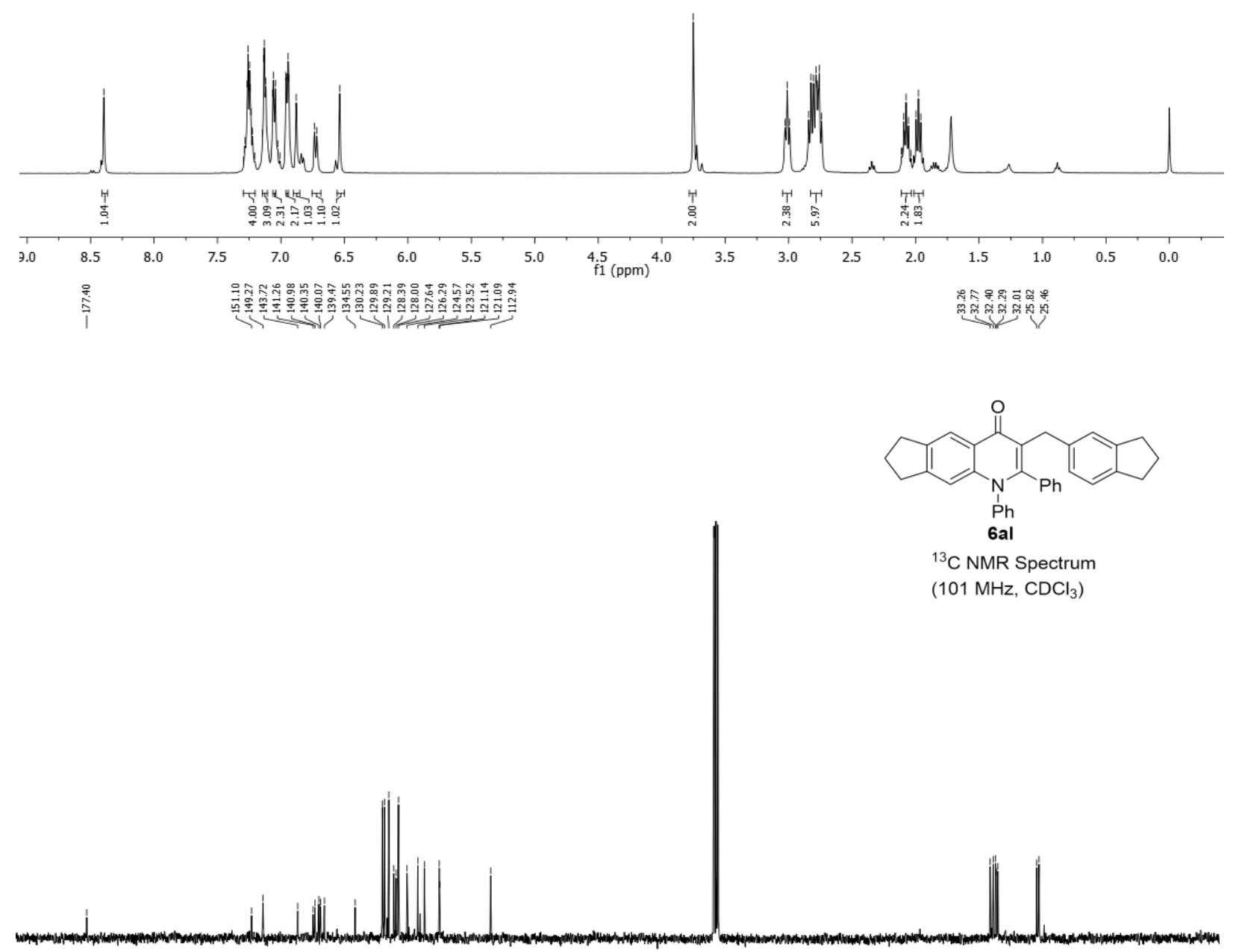


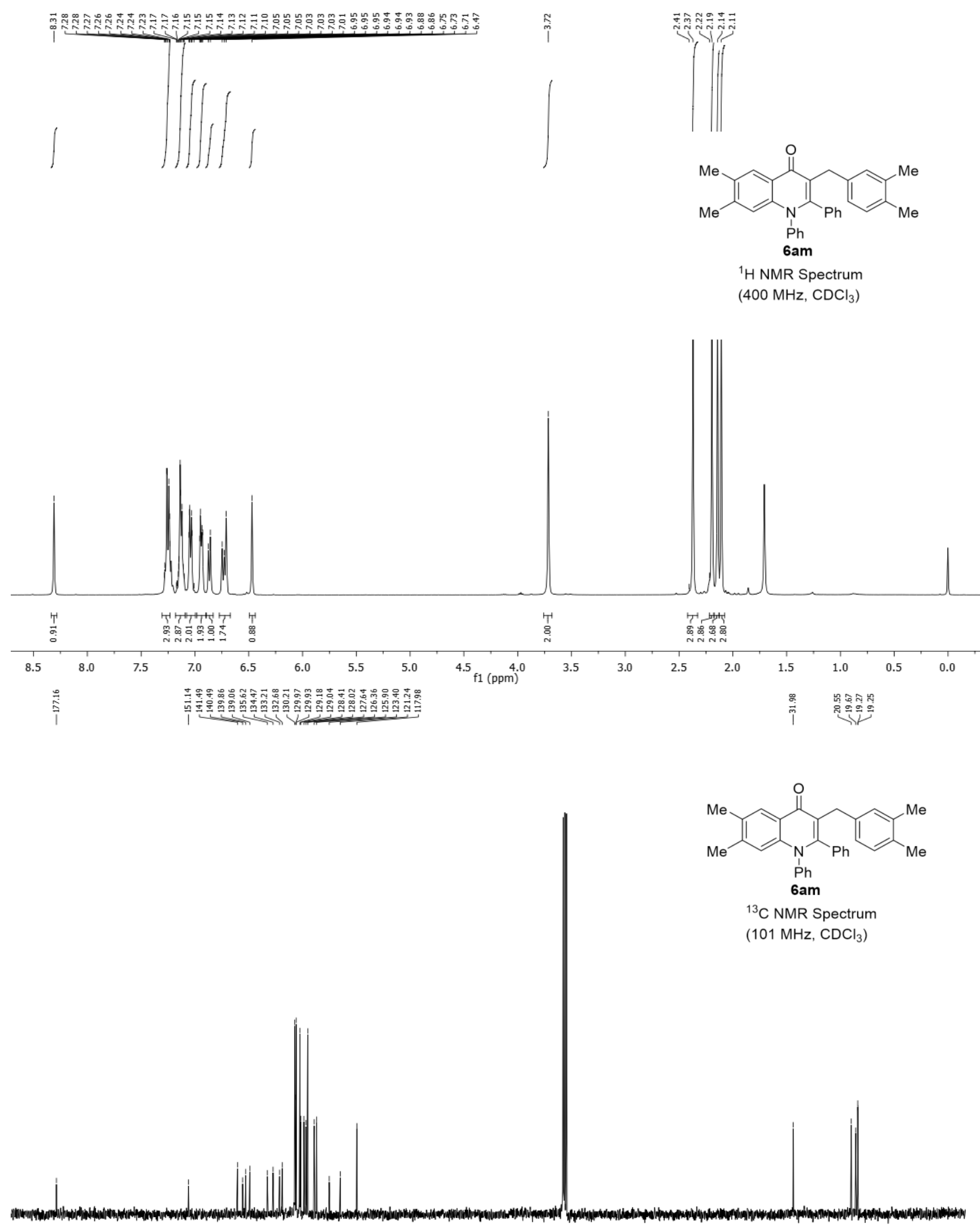

\begin{tabular}{|c|c|c|c|c|c|c|c|c|c|c|c|c|c|c|c|c|c|}
\hline 180 & 170 & 160 & 150 & 140 & 130 & 120 & 110 & 100 & $\begin{array}{c}90 \\
\mathrm{f} 1(\mathrm{ppm})\end{array}$ & 80 & 70 & 60 & 50 & 40 & 30 & 20 & 10 \\
\hline
\end{tabular}



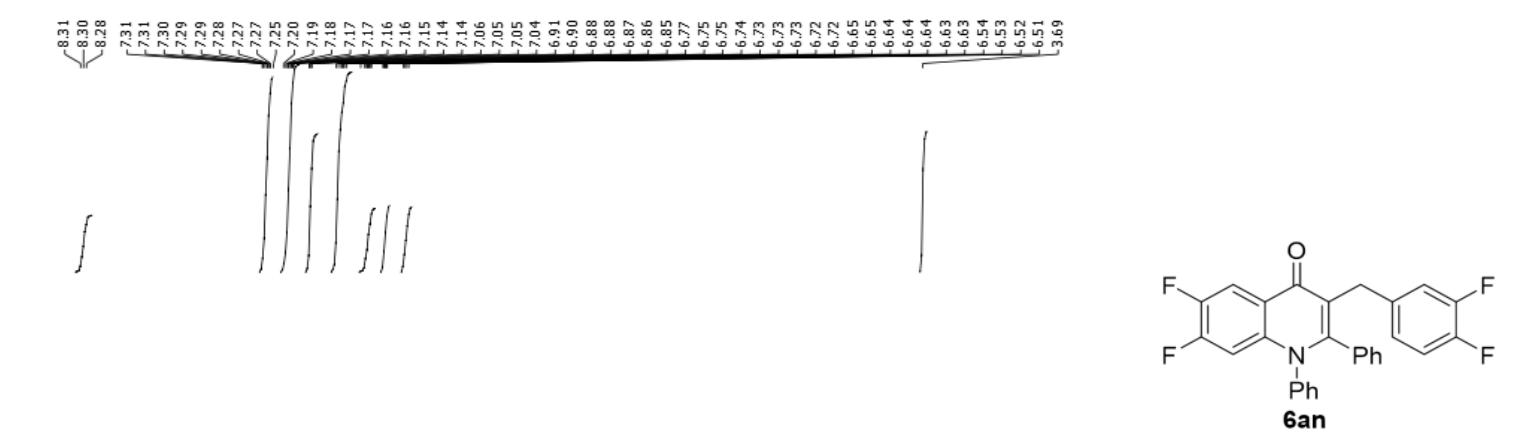

${ }^{1} \mathrm{H}$ NMR Spectrum $\left(400 \mathrm{MHz}, \mathrm{CDCl}_{3}\right.$ )
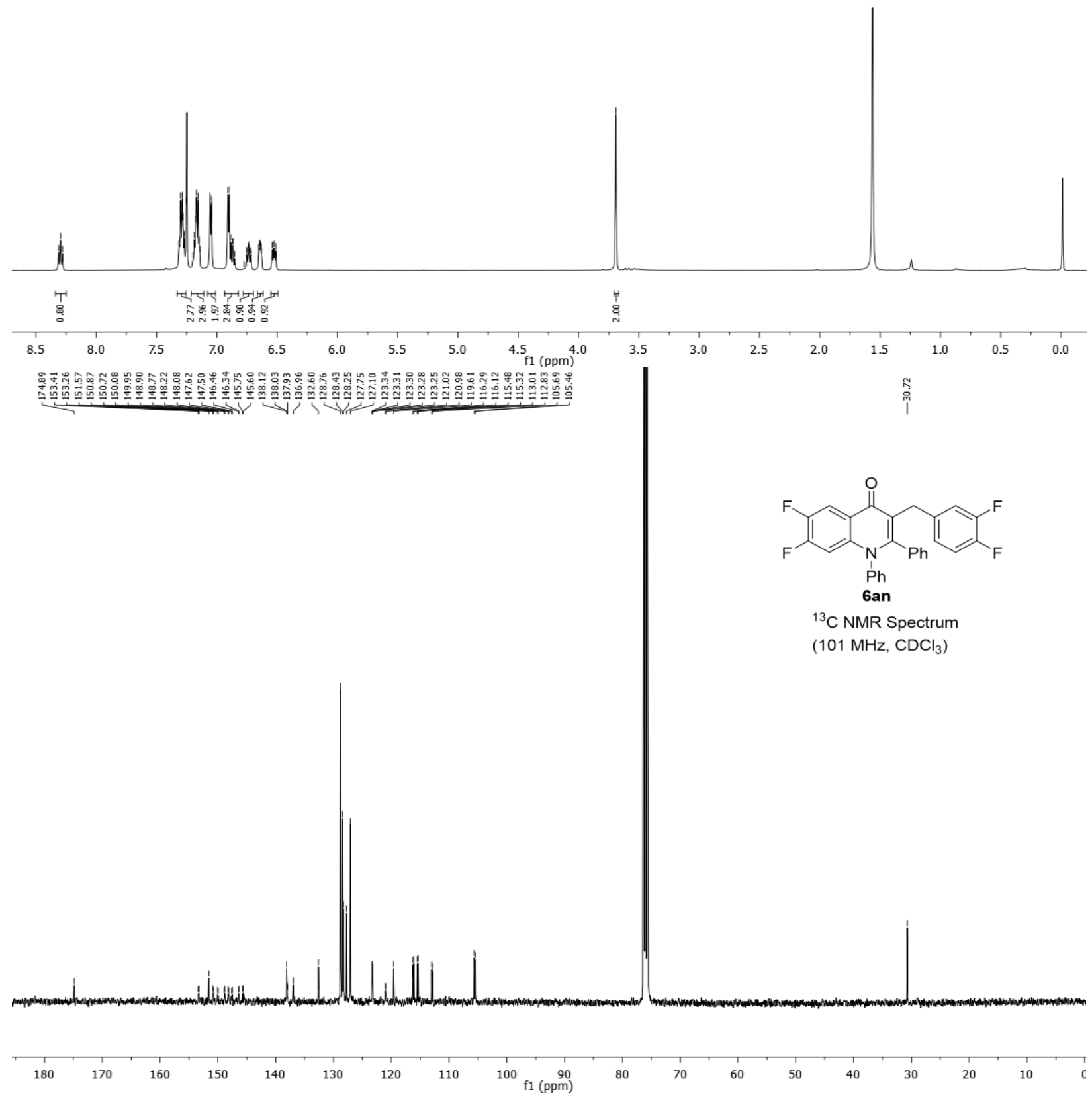


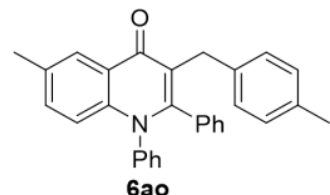

mixture of 4 isomers ${ }^{1} \mathrm{H}$ NMR Spectrum (400 $\mathrm{MHz}, \mathrm{CDCl}_{3}$ )
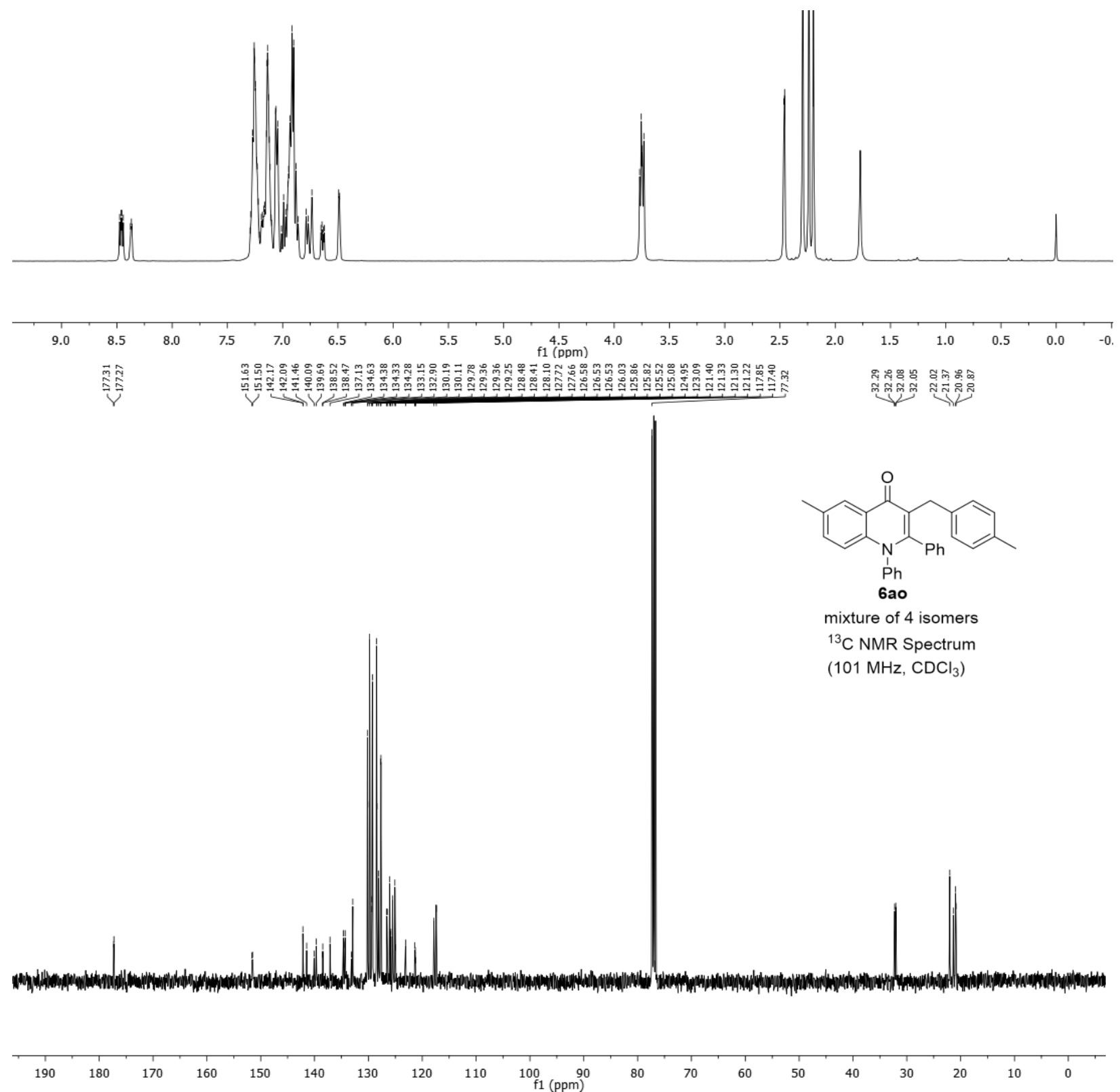


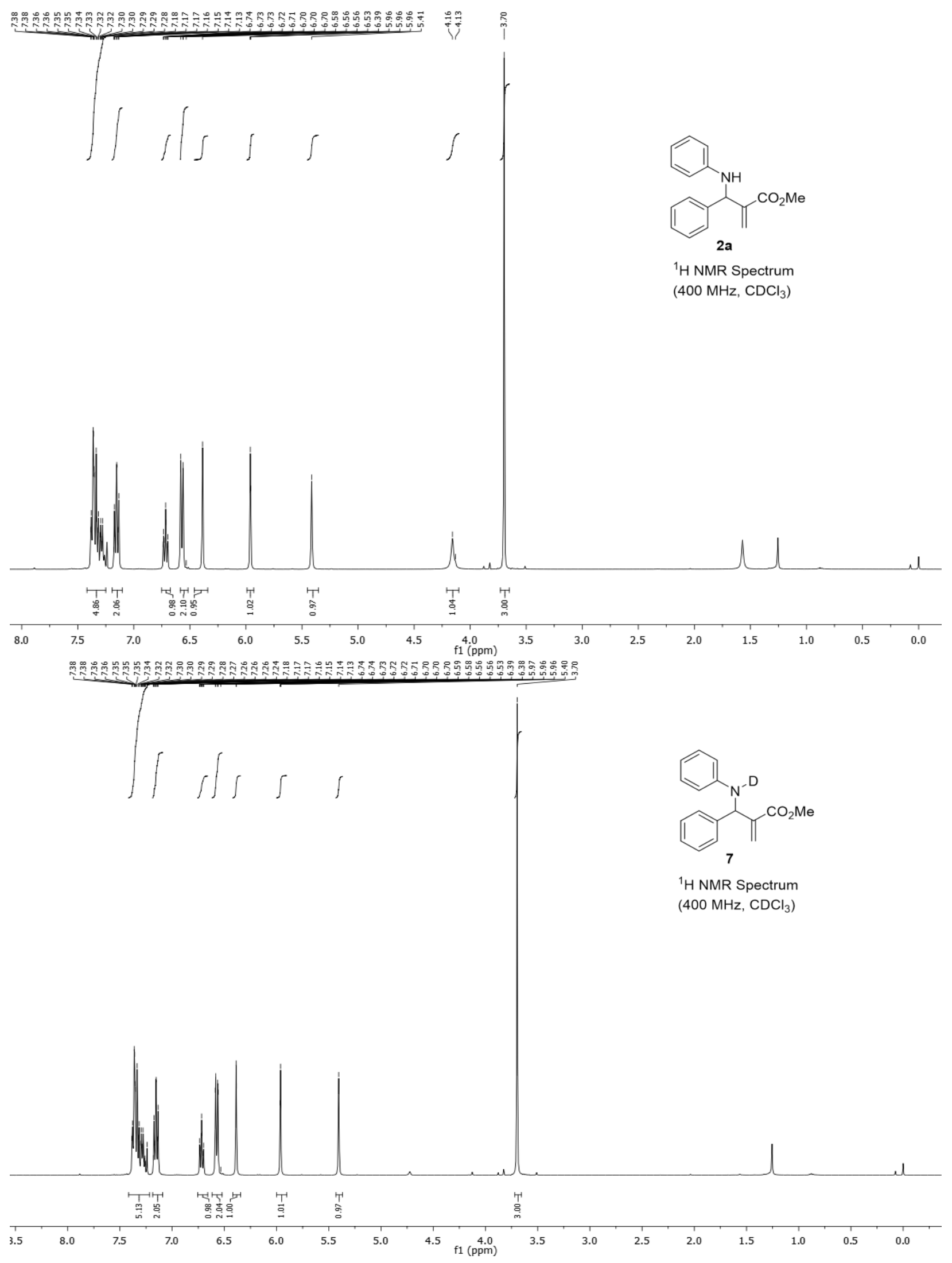



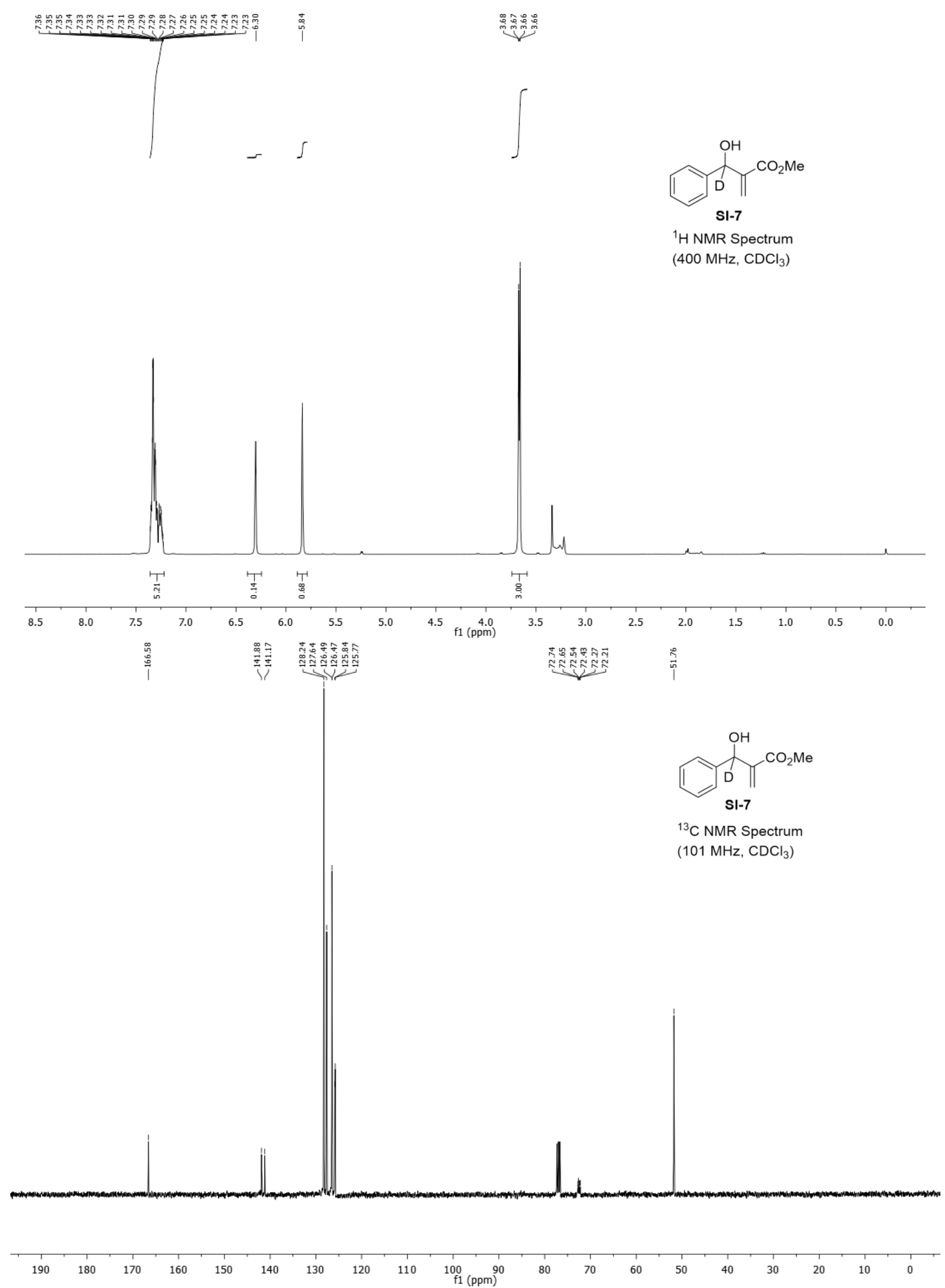


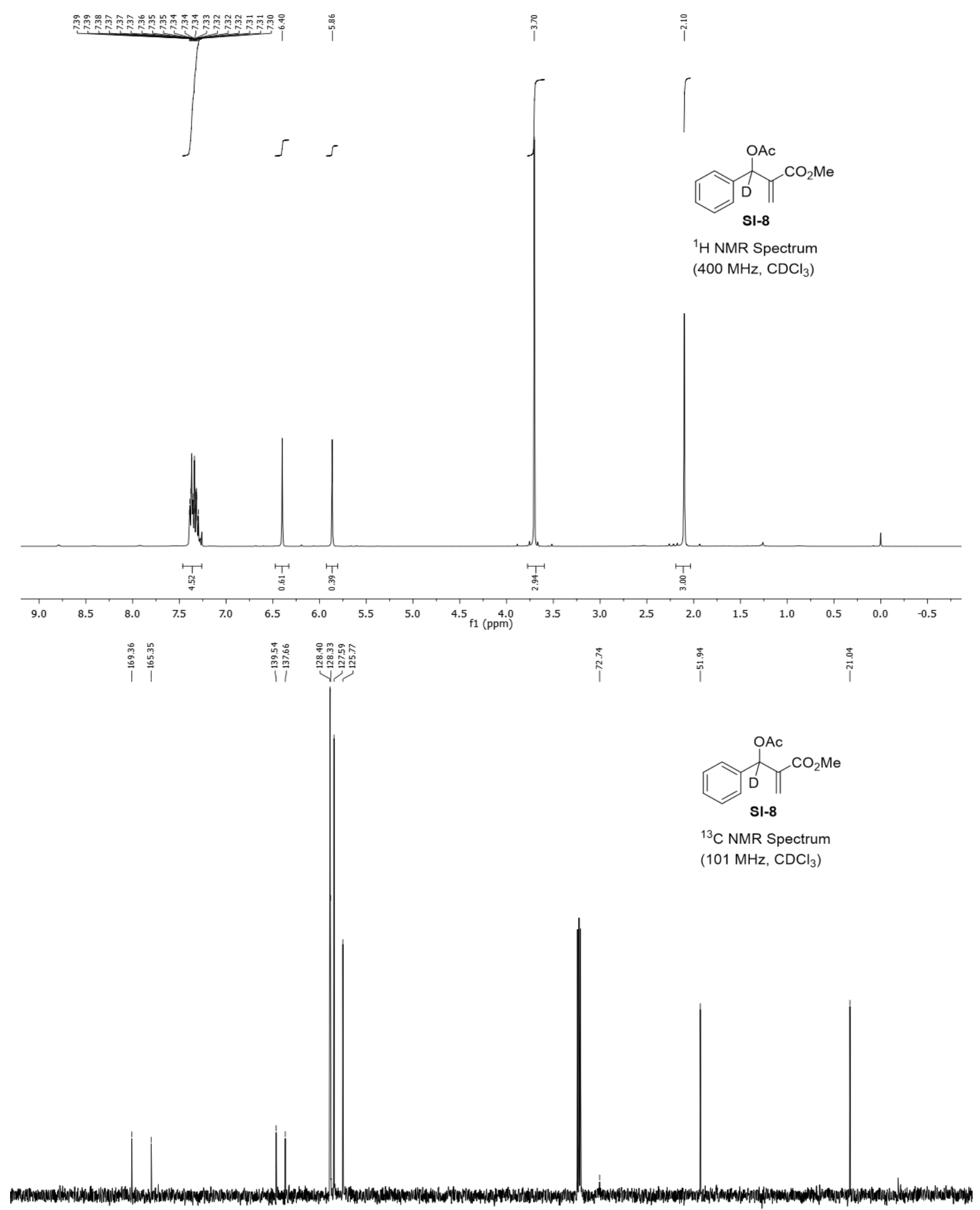

\begin{tabular}{|c|c|c|c|c|c|c|c|c|c|c|c|c|c|c|c|c|c|c|}
\hline 1 & 1 & 1 & 1 & 1 & 1 & 1 & 1 & 1 & 1,1 & 1 & 1 & 1 & 1 & 1 & $T$ & 1 & 1 & \\
\hline 190 & 180 & 170 & 160 & 150 & 140 & 130 & 120 & 110 & $\stackrel{100}{\mathrm{f} 1(\mathrm{ppm})}{ }^{90}$ & 80 & 70 & 60 & 50 & 40 & 30 & 20 & 10 & 0 \\
\hline
\end{tabular}



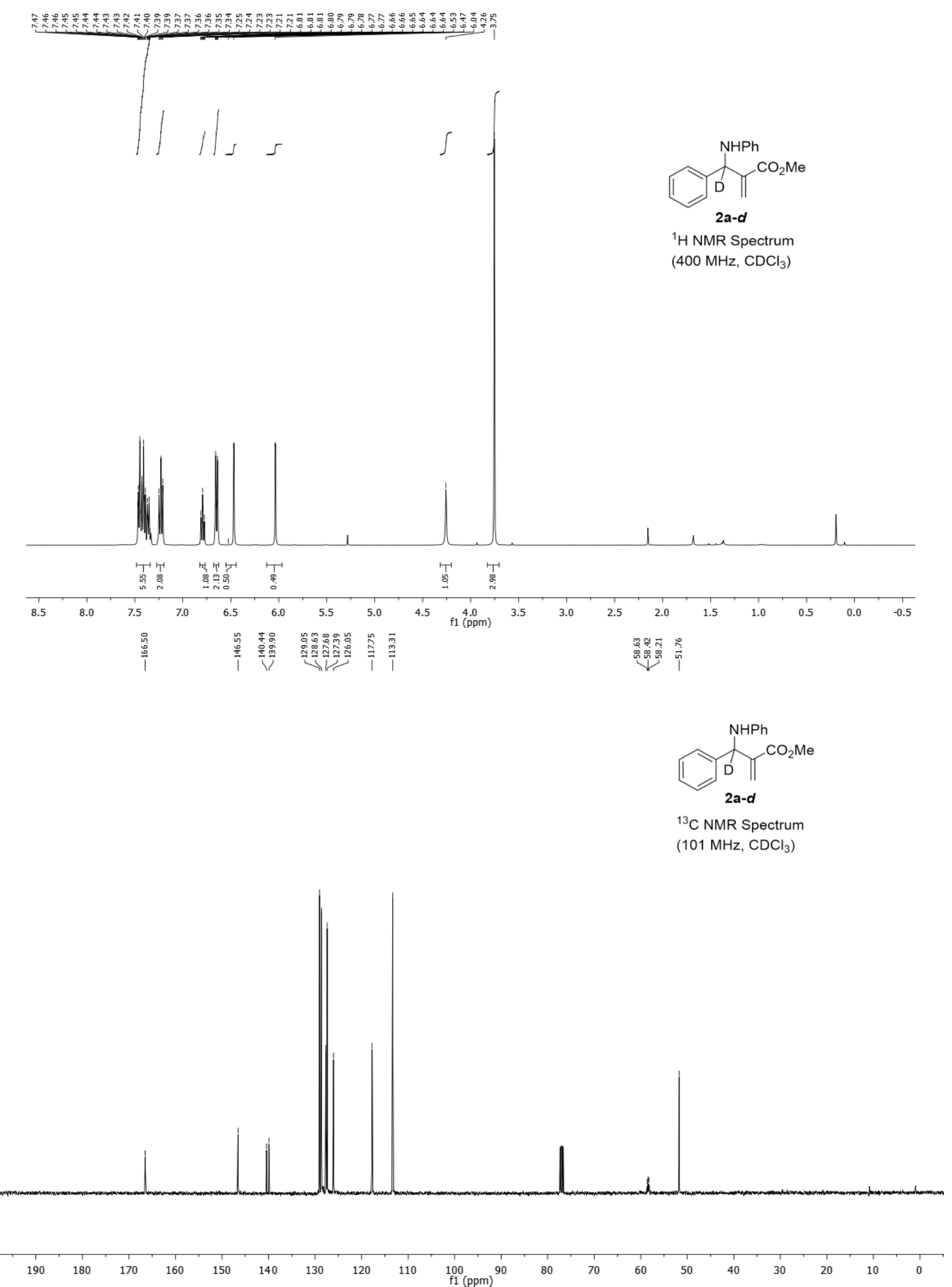

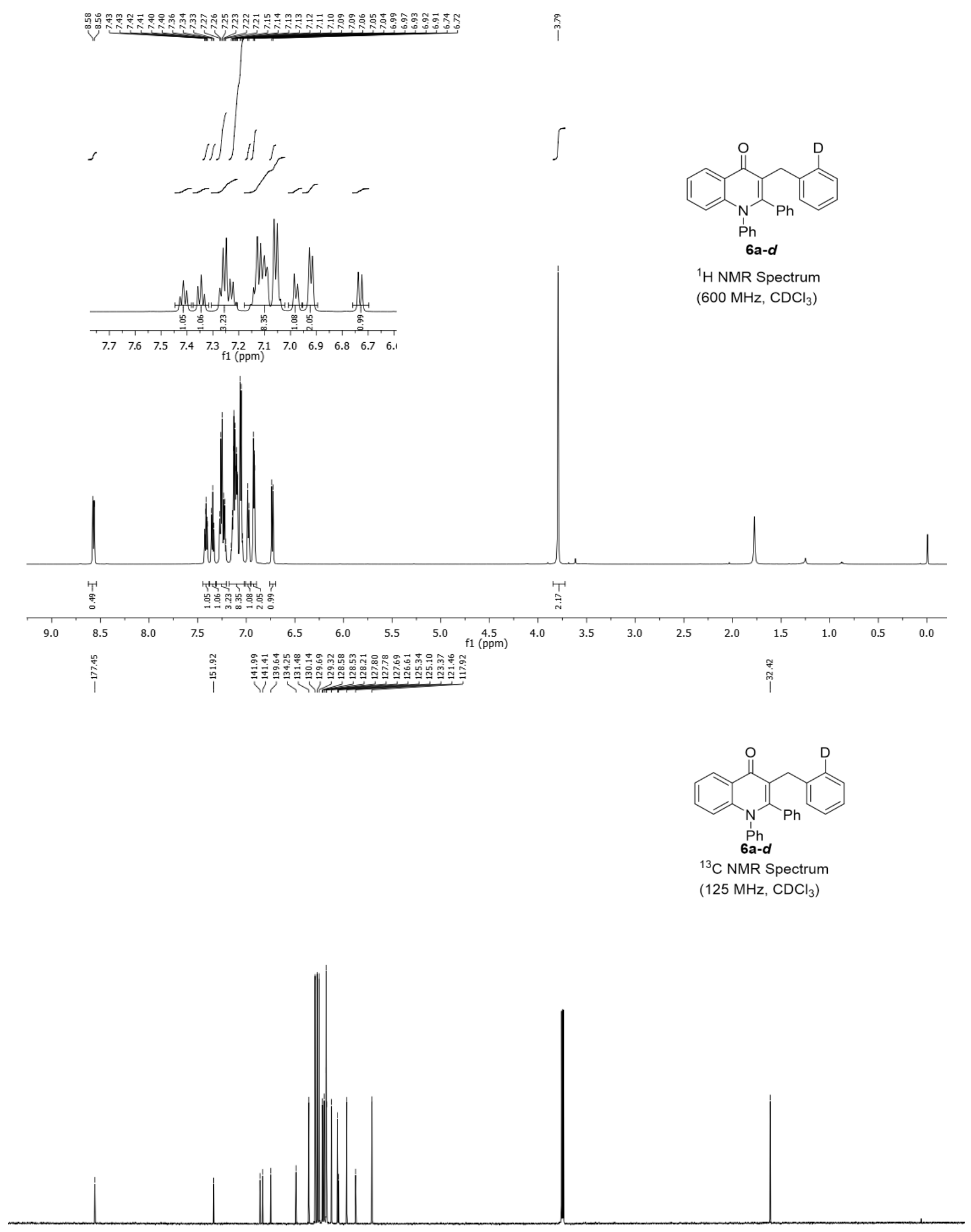

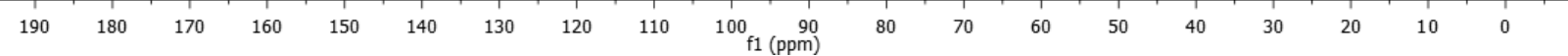



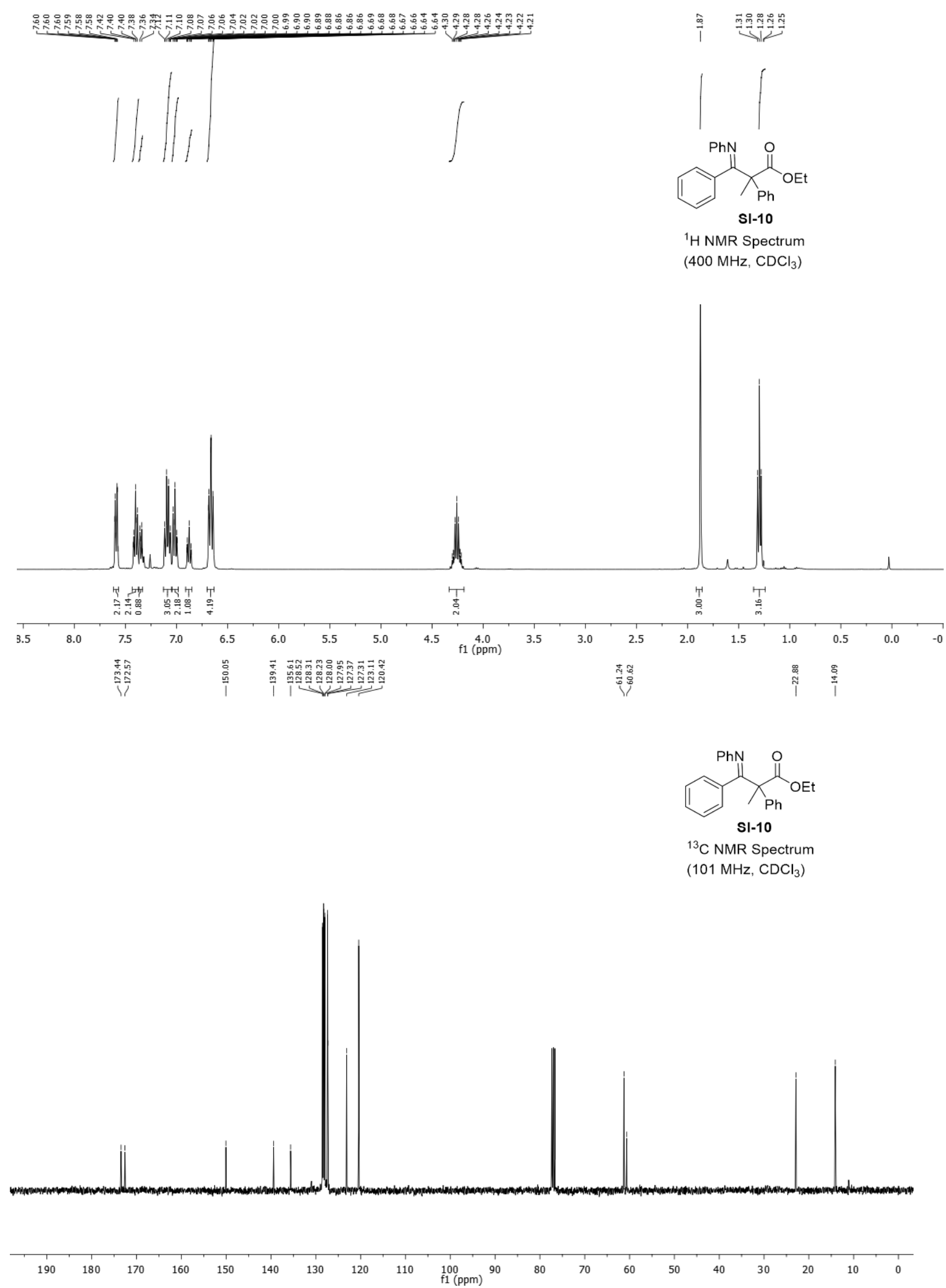